\title{
Resolving the role of genetic defects and mtDNA copy number in mitochondrial disease and development
}

Citation for published version (APA):

Kamps, R. (2020). Resolving the role of genetic defects and mtDNA copy number in mitochondrial disease and development. [Doctoral Thesis, Maastricht University]. Maastricht University. https://doi.org/10.26481/dis.20201120rk

Document status and date:

Published: 01/01/2020

DOI:

10.26481/dis.20201120rk

Document Version:

Publisher's PDF, also known as Version of record

\section{Please check the document version of this publication:}

- A submitted manuscript is the version of the article upon submission and before peer-review. There can be important differences between the submitted version and the official published version of record.

People interested in the research are advised to contact the author for the final version of the publication, or visit the DOI to the publisher's website.

- The final author version and the galley proof are versions of the publication after peer review.

- The final published version features the final layout of the paper including the volume, issue and page numbers.

Link to publication

\footnotetext{
General rights rights.

- You may freely distribute the URL identifying the publication in the public portal. please follow below link for the End User Agreement:

www.umlib.nl/taverne-license

Take down policy

If you believe that this document breaches copyright please contact us at:

repository@maastrichtuniversity.nl

providing details and we will investigate your claim.
}

Copyright and moral rights for the publications made accessible in the public portal are retained by the authors and/or other copyright owners and it is a condition of accessing publications that users recognise and abide by the legal requirements associated with these

- Users may download and print one copy of any publication from the public portal for the purpose of private study or research.

- You may not further distribute the material or use it for any profit-making activity or commercial gain

If the publication is distributed under the terms of Article $25 \mathrm{fa}$ of the Dutch Copyright Act, indicated by the "Taverne" license above, 
Resolving the Role of Genetic Defects and mtDNA

Copy Number in Mitochondrial Disease and Development

Ricky Kamps 
(C) copyright Ricky Kamps, Maastricht 2020

Printing: ProefschriftMaken || www.proefschriftmaken.nl

ISBN 978-94-6423-031-4

All rights reserved. No part of this publication may be reproduced, stored in a retrieval system or transmitted, in any form or by any means, electronic, mechanical, photocopying, recording or otherwise, without prior permission of the author or the copyright-owning journals for previous published chapters. 


\section{Resolving the Role of Genetic Defects and mtDNA Copy Number in Mitochondrial Disease and Development}

\section{PROEFSCHRIFT}

ter verkrijging van de graad van doctor aan de Universiteit Maastricht, op gezag van de Rector Magnificus, Prof. dr. Rianne M. Letschert volgens het besluit van het College van Decanen, in het openbaar te verdedigen op vrijdag 20 november 2020 om 14.00 uur

door

Ricky Kamps

Geboren op 22 december 1972 te Heerlen 


\section{Promotor:}

Prof. dr. H.J.M. Smeets

\section{Copromotor:}

Dr. F.H.J. van Tienen

\section{Beoordelingscommissie:}

Prof. dr. C.G. Faber (voorzitter)

Prof. dr. R. Horvath (University of Cambridge, United Kingdom)

Prof. dr. C. Spits (Vrije Universiteit Brussel, Belgium)

Prof. dr. M.K.C. Hesselink

Prof. dr. S.E. Köhler 


\section{Contents}

Abbreviations

Chapter 1 General Introduction and Outline of the Thesis

Part I Whole Exome Sequencing a Successfull Strategy to Identify

Chapter 2 Next-Generation Sequencing in Genetic Diagnosis, Risk Prediction and Disease Classification

Chapter 3 Germ-line Variants Identified by Next-Generation Sequencing in a Panel of Estrogen and Cancer Associated Genes Correlate with Poor Clinical Outcome in Lynch Syndrome Patients

Chapter 4 Exome Sequencing Reveals a Novel Moroccan

Founder Mutation in SLC19A3 as a New Cause of Early-Childhood

Fatal Leigh Syndrome

Chapter 5 Genetic Defects in mtDNA-encoded Protein Translation Cause Paediatric, Mitochondrial Cardiomyopathy with Early-onset Brain Disease

Chapter 6 Tfam Depletion Leads to a Strong Reduction in mtDNA Copy Number OXPHOS Deficiency and Developmental Abnormalities in Zebrafish Embryos 
Nederlandse samenvatting $\quad 231$

$\begin{array}{lr}\text { Acknowledgements } & 239\end{array}$

Dankwoord $\quad 241$

Curriculum Vitae $\quad 243$

List of Publications $\quad 247$ 


\section{Abbreviations}

AARS2

ACAD 9

ADP

ANT

ART

ATP

B2M

C100rf2

C12orf65

CI

CII

CIII

CIV

CV

CHIA1, 2, 3

COX

CYP450

DARS2

DNA2

D-Loop

ECG

ELAC2

GNAT2

GPX1

GTP

LS

LHON

MELAS

MGME1

MO
Alanyl-tRNA synthetase 2

Acyl-CoA Dehydrogenase family member 9

Adenosine diphosphate

Adenine nucleotide transporter

Assisted reproductive technology

Adenosine triphosphate

Beta 2-microglobuline

Chromosome 10 open reading frame 2

Chromosome 12 open reading frame 65

Complex I

Complex II

Complex III

Complex IV

Complex V

Chitinase Acidic 1, 2, 3

Cytochrome c oxidase

Cytochrome P450

Aspartyl-tRNA synthetase

DNA replication helicase 2

Displacement loop

Electrocardiogram

ElaC Ribonuclease Z 2

G Protein Subunit Alpha Transducin 2

Glutathione Peroxidase 1

Guanosinetrifosfaat

Leigh syndrome

Leber's hereditary optic neuropathy

Mitochondrial encephalopathy lactic acidosis and stroke like episodes

Mitochondrial genome maintenance exonuclease 1

Morpholino Oligonucleotides 


\begin{tabular}{|c|c|}
\hline mRNA & Messenger RNA \\
\hline MRI & Magnetic resonance spectroscopy \\
\hline MRPL3 & Mitochondrial ribosomal protein $\mathrm{L} 3$ \\
\hline MRPL44 & Mitochondrial ribosomal protein $\mathrm{L} 44$ \\
\hline mtDNA & Mitochondrial DNA \\
\hline MTATP6 & Mitochondrial encoded ATP synthetase membrane 6 \\
\hline MTATP8 & Mitochondrial encoded ATP synthetase membrane 8 \\
\hline MTCO1 & Mitochondrial encoded cytochrome c oxidase subunit 1 \\
\hline MTCO2 & Mitochondrial encoded cytochrome c oxidase subunit 2 \\
\hline MTCO3 & Mitochondrial encoded cytochrome c oxidase subunit 3 \\
\hline MTCYB & Mitochondrial encoded cytochrome B \\
\hline MTND1 & NADH dehydrogenase, subunit 1 \\
\hline MTND2 & NADH dehydrogenase, subunit 2 \\
\hline MTND3 & NADH dehydrogenase, subunit 3 \\
\hline MTND4 & NADH dehydrogenase, subunit 4 \\
\hline MTND4L & NADH dehydrogenase, subunit 4 ligand \\
\hline MTND5 & NADH dehydrogenase, subunit 5 \\
\hline MTND6 & NADH dehydrogenase, subunit 6 \\
\hline mTERF & Mitochondrial transcription termination factor \\
\hline MTFMT & Mitochondrial methionyl-tRNA formyltransferase \\
\hline MTO1 & tRNA modification and protein synthesis \\
\hline MPV17 & Mitochondrial inner membrane protein MPV17 \\
\hline MRT & Mitochondrial replacement therapy \\
\hline $\mathrm{NADH}$ & Nicotinamide adenine dinucleotide \\
\hline nDNA & Nuclear DNA \\
\hline NGS & Next-generation sequencing \\
\hline NME1 & Nucleoside diphosphatase kinase 1 \\
\hline NME2 & Nucleoside diphosphatase kinase 2 \\
\hline OPA1 & Mitochondrial Dynamin Like GTPase \\
\hline OXCT1 & 3-Oxacid CoA Transferase 1 \\
\hline OXPHOS & Oxidative phosphorylation \\
\hline
\end{tabular}




\begin{tabular}{|c|c|}
\hline PCR & Polymerase chain reaction \\
\hline PCS & Preconception carrier screening \\
\hline PDE6C & Phosphodiesterase 6C \\
\hline PGD & Preimplantation genetic diagnostics \\
\hline PND & Prenatal diagnostics \\
\hline POLG1 & Polymerase Gamma 1 \\
\hline POLG2 & Polymerase Gamma 2 \\
\hline POLRMT & mRNA polymerase \\
\hline QPCR & Quantitative polymerase chain reaction \\
\hline QRSL1 & Glu-tRNA synthetase \\
\hline RNA-Seq & RNA-sequencing \\
\hline rRNA & Ribosomal RNA \\
\hline RRM2B & Ribonucleotide reductase M2 B \\
\hline STDEV & Standard deviation \\
\hline SEM & Standard error mean \\
\hline SLC19A2 & Solute carrier family 19 member 2 \\
\hline SLC19A3 & Solute carrier family 19 member 3 \\
\hline SUCLA1 & Succinate CoA Ligase ADP forming alpha subunit \\
\hline SUCLA2 & Succinate CoA Ligase ADP forming beta subunit \\
\hline SUCLG1 & Succinate CoA Ligase alpha subunit \\
\hline SURF1 & Surfeit Locus protein \\
\hline TCA & Tricarboxylic acid \\
\hline TMEM126B & Complex I assembly factor \\
\hline TFAM & Transcription factor $\mathrm{A}$ \\
\hline TFB2M & Mitochondrial transcription factor B2 \\
\hline TK1 & Thymidine kinase 1 \\
\hline TK2 & Thymidine kinase 2 \\
\hline tRNA & Transfer RNA \\
\hline TRMT & Mitochondrial 5-Methylaminomethyl-2-Thiouridylate Methyltransferase \\
\hline TP & Thymidine phosphorylase \\
\hline TYMP & Thymidine phosphorylase \\
\hline
\end{tabular}


Abbreviations

TSFM Ts translational elongation factor, mitochondrial

TUFM

Tu translational elongation factor, mitochondrial

VUS

Variant of unclassified significance

WES

Whole-exome sequencing

WGS

Whole-genome sequencing 



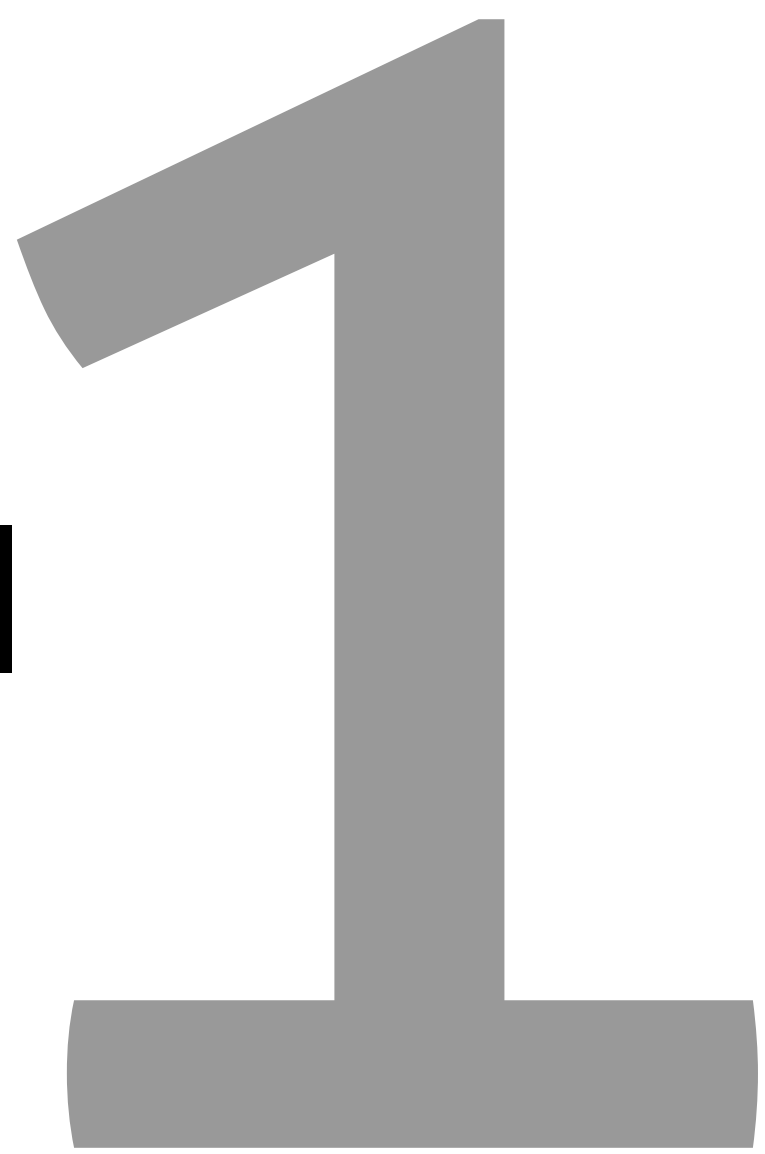


General Introduction

and Outline of the Thesis 


\section{Introduction: Mitochondria}

In general, mitochondria are considered the powerhouses of the cell [1]. Mitochondria are organelles, which convert sugars, fats and proteins inside the mitochondrial double membrane into adenosine triphosphate (ATP), the cellular source of energy. Mitochondria are present in varying numbers per cell type, tissue, and organs, usually depending on their energy requirements. Originally, mitochondria were free-living prokaryotes but more than a billion years ago an endosymbiotic event made the free-living bacterium part of what became a eukaryotic cell [2]. During evolution the parasite changed into a central mutualistic organelle, we nowadays know as mitochondria, which was accompanied with strong reduction in gene number and genome size. Only a small amount of genetic material remained in the mitochondria, which is the current mitochondrial DNA (mtDNA) [3]. Although energy production is the main function of mitochondria, other cellular processes, such as heme synthesis, cellular apoptosis, and calcium homeostasis, are also carried out by mitochondria [4].

\subsection{Mitochondrial Energy Production}

Several processes generate ATP from glucose, such as glycolysis, the tricarboxylic acid (TCA) or Krebs cycle, the respiratory chain and ATP synthesis (Figure 1). Glycolysis generates 2 ATPs during the oxidation of glucose to pyruvate. Pyruvate is being transported into the mitochondrial matrix and converted into Acetyl-CoA, which is a substrate for the TCA cycle. The TCA cycle produces energy-rich metabolites, such as $\mathrm{FADH}_{2}, \mathrm{NADH}, \mathrm{GTP}$, and 2 ATPs. At the mitochondrial inner membrane, electrons from $\mathrm{NADH}$ and $\mathrm{FADH}_{2}$ pass through the electron transport chain to oxygen, which is reduced to water. Electrons from NADH are transferred to Complex I (CI) and electrons from $\mathrm{FADH}_{2}$ to Complex II (CII), followed in both cases by transfer to Co-enzyme Q (CoQ). CoQ transports the electrons to Complex III (CIII) and via cytochrome c to Complex IV (CIV). The function of the electron transport chain is to generate a transmembrane proton electrochemical gradient. This gradient is used by the ATP synthase complex, often referred to as complex $\mathrm{V}$, to generate 32 ATP from ADP via oxidative phosphorylation (Figure 1). ATP transport to the cytosol is processed via the ADP/ATP transporter, better known as adenine nucleotide transporter (ANT) [5]. 
Glycolysis in the Cytoplasm

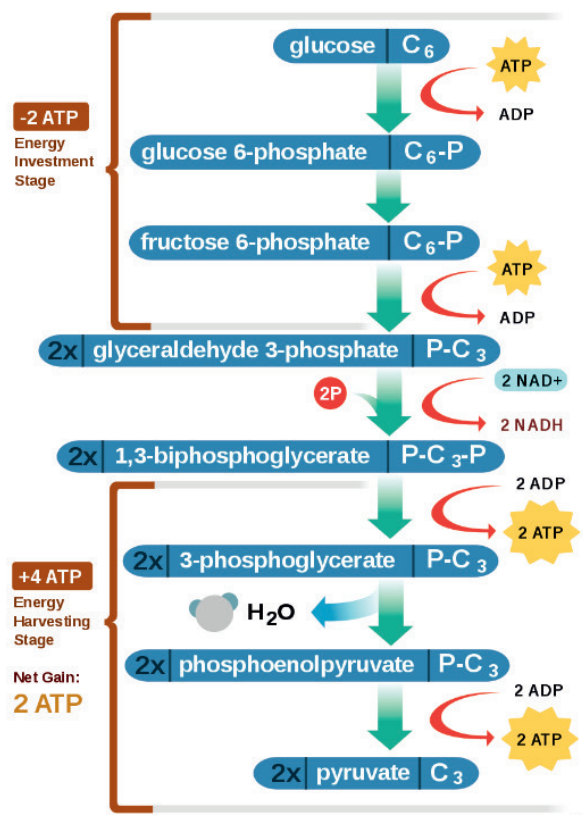

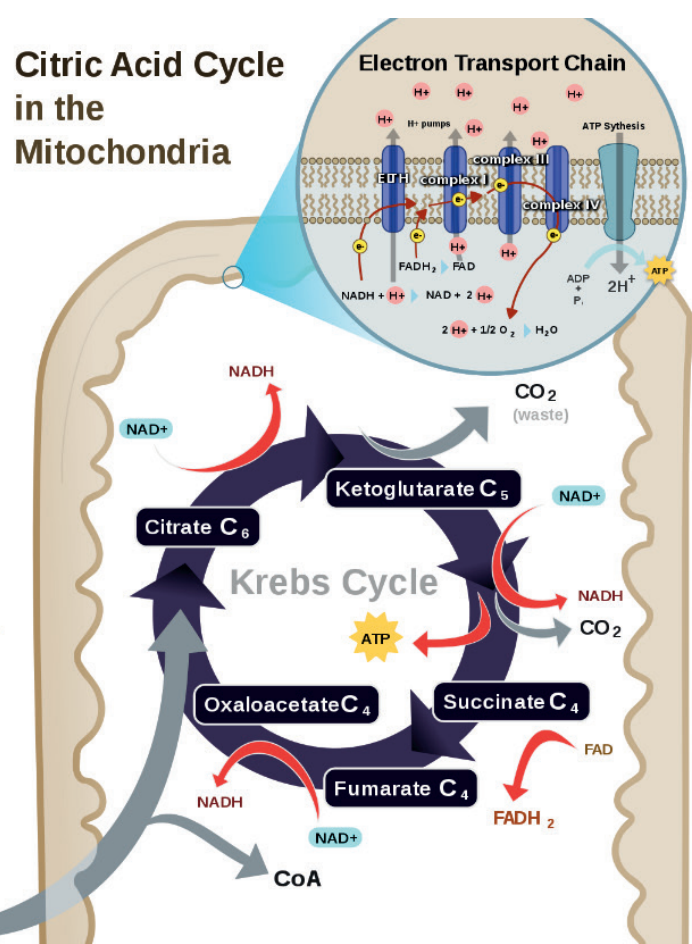

Figure 1. Cellular respiration and mitochondrial energy production. The primary role of mitochondria is the production of cellular energy (ATP) by a process called oxidative phosphorylation. In total, each glucose molecule undergoing cellular respiration produces 38 ATP molecules: 2 ATPs from glycolysis, 2 ATPs from Krebs cycle, and 34 ATPs from oxidative phosphorylation. By RegisFrey - Own work, CC BY-SA 3.0, https://commons. wikimedia.org/w/index.php?curid=4389845.

\subsection{The Structure of the Mitochondria}

Mitochondria contain an outer and inner membrane composed of a phospholipid bilayer and proteins. The outer mitochondrial membrane encloses the entire organelle, which contains protein import machineries and protein channels that allow transport of small molecules and proteins into the mitochondrial intermembrane space. The inner mitochondrial membrane, which is folded into a number of cristae to increase the surface area, contains multiple proteins such as the respiratory chain complexes, Adenosine Triphosphate (ATP) synthase, assembly proteins, and metabolite import machineries [6]. The space enclosed by the mitochondrial inner membrane is called the mitochondrial matrix. This matrix contains enzymes, tRNAs, mitochondrial ribosomes, and copies of mtDNA. Additionally, also other enzymes, involved in the urea cycle, in branched chain 
amino acid metabolism, and in heme synthesis are present in the mitochondrial matrix [6].

\subsection{The Mitochondrial and the Nuclear Genome}

The mitochondria have a dual genetic origin, with a small part of the genetic information, being present in the mtDNA and the vast majority in the nuclear DNA. The mtDNA is a $16,569 \mathrm{~kb}$ circular double-stranded molecule, containing 37 genes (22 transfer RNAs (tRNA) genes, 2 ribosomal RNAs (rRNA) genes and 13 protein encoding genes, which are all structural subunits of the enzyme complexes of the respiratory chain, being 7 subunits of Complex I (CI), 1 of Complex III (CIII), 3 Complex IV (CIV), and 2 of Complex V (CV), (Figure 2). The majority of the structural proteins of the OXPHOS complexes and the proteins involved in mtDNA replication, transcription, translation, in assembly of the OXPHOS protein complexes, maintenance, and in mitochondrial quality control are encoded by around 1500 nDNA genes [6]. The proteins are targeted to the mitochondria by specific import sequences. An inventory of human mitochondrial genes is represented in the MitoCarta2.0 inventory, comprising a still incomplete collection of 1158 nuclear and mtDNA genes encoding proteins with a likely mitochondrial function [7]. Nuclear mitochondrial genes are inherited from both parents, but the mtDNA shows exclusive maternal transmission, as the paternal mtDNA in the sperm is eliminated upon fertilization. 


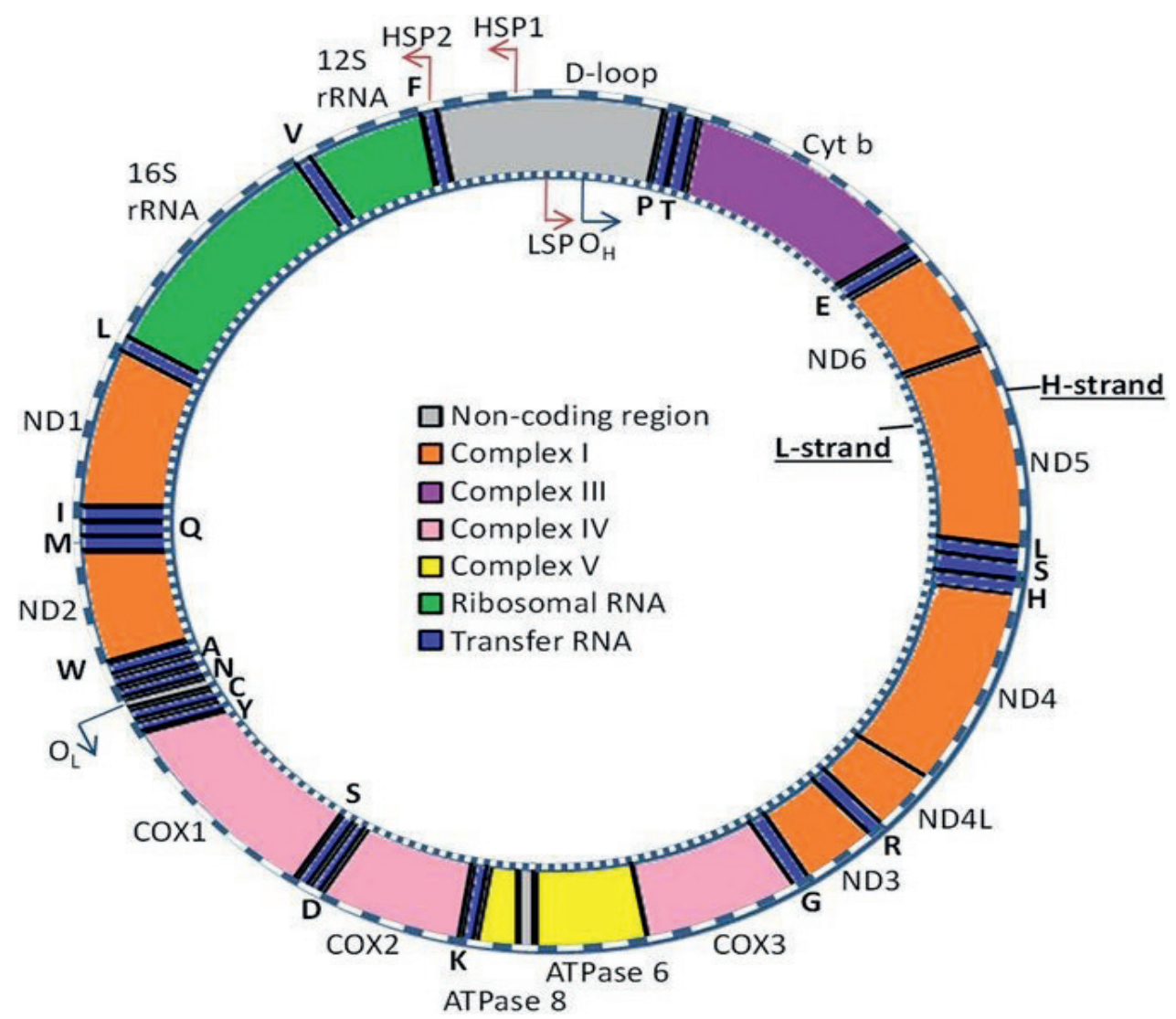

Figure 2. The mitochondrial DNA (mtDNA). The human circular mitochondrial DNA consists of $16,569 \mathrm{bp}$, encoding 22 tRNA's, 2 rRNA's, and 13 core-subunits of the OXPHOS complexes. Complex I genes are orange, complex III genes are purple, complex IV genes are rose, complex $\mathrm{V}$ genes are yellow, rRNA genes are green, tRNA genes are blue and black border lines. Adapted from Van der Wijst, GPM. et al., Scientific Reports 7, Article number: 177 (2017).

\subsection{Mitochondrial DNA Transcription, Translation, and Replication}

Mitochondrial DNA replication is under control of nuclear encoded proteins, which are involved in the unwinding of the double stranded mtDNA, the synthesis of the mtDNA strands and the supply of nucleotides (Figure 3 ). The mtDNA contains a non-coding control region which is $1000 \mathrm{bp}$ long. This region, also called displacement loop (D-Loop), forms a three-stranded structure which functions as start-site of replication. mtDNA replication is linked to mtDNA transcription, which 
produces the 37 RNAs (ribosomal, 22 transfer, and 13 messenger RNAs). Mitochondrial transcription can originate from three sites: one L-strand promoter, and two $\mathrm{H}$-strand promoters and requires mRNA polymerase (POLRMT), transcription factor A (TFAM), and mitochondrial transcription factor B2 (TFB2M) (Figure 2). Transcription of mtDNA needs TFAM, which binds to the promoters on the heavy and the light strands of the mtDNA, but also covers the mtDNA as a sort of protective histone [8]. The genes are transcribed as a polycistronic RNAs, from which the specific tRNA, rRNA and mRNA are being cleaved [8]. Termination of transcription is regulated by specific termination factors, including mTERF.

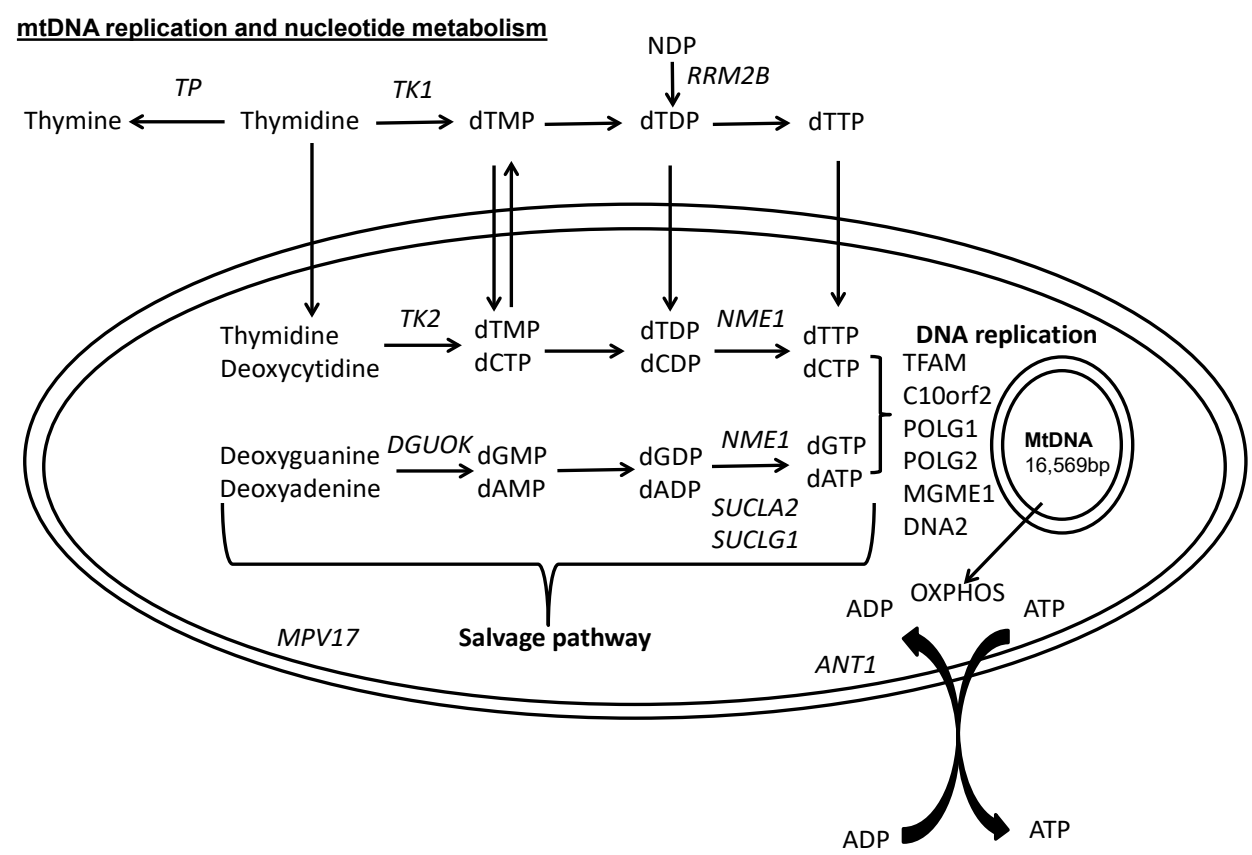

Figure 3. Mitochondrial replication and nucleotide metabolism. A schematic overview of the pathways involved in mitochondrial replication and nucleotide metabolism. Adapted from Camara Y. et al. Feeding the deoxyribonucleoside salvage pathway to rescue mitochondrial DNA, Drug discovery today. 2013, Doi:10.1016/j.drudis.2013.06.009. 
For translation of the mtDNA-encoded proteins, mitochondrial transfer RNAs are charged with the corresponding amino acids by nuclear-encoded amino-acyl tRNA synthetases (e.g. AARS2, DARS2, EARS2 etc.). The mitochondrial ribosomes contain mtDNA encoded rRNA/tRNAs and nuclear encoded ribosomal proteins (e.g. MRPL3, MRPL44), translation elongation factors (e.g. GFM1, TSFM, and TUFM), recycling and release factors (e.g. C12orf65, GFM2), and catalyzing maintenance enzymes (e.g. MTFMT, MPV17) required for synthesis of the 13 coresubunits of the OXPHOS proteins (Figure 4).

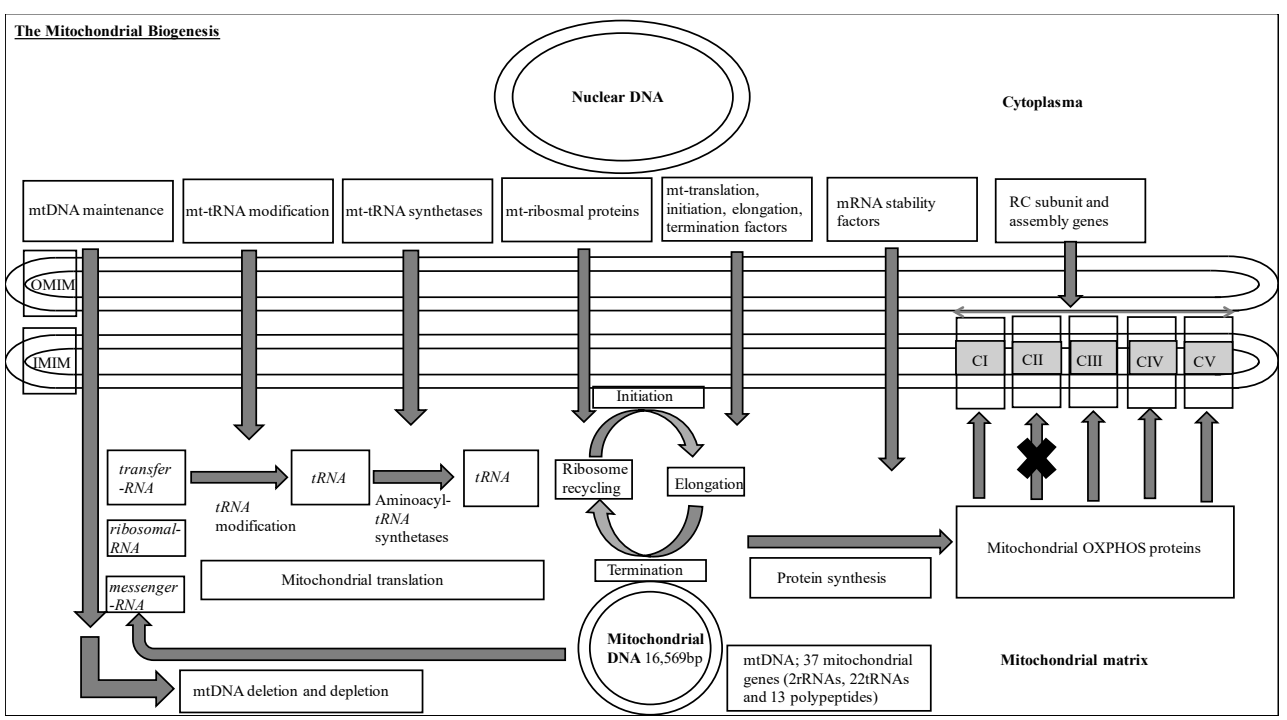

Figure 4. Mitochondrial biogenesis. A schematic overview of the pathways involved in mitochondrial biogenesis. Adapted from Kamps R. et al., Eur J Hum Genet. 2018 EJHG.

\subsection{Mitochondrial Disease}

\subsubsection{Clinical Heterogeneity}

Mitochondrial diseases are the most common genetic metabolic diseases, affecting approximately 1 in 5,000 individuals. Mitochondrial diseases form a clinically and genetically heterogeneous group of disorders, which generally manifest in tissues or organs with a high-energy requirement. Mitochondrial disease can affect multiple organs and lead to specific syndromes, like MELAS Mitochondrial Encephalomyopathy Lactic Acidosis and Stroke-like episodes, but they can also affect only a single organ or system, like blindness, in Leber hereditary optic 
neuropathy (LHON). Also, more general manifestations, like sensorineural hearing loss, seizures, ataxia, myopathy, diabetes or cardiomyopathy [8, 9], can be part of the spectrum of mitochondrial diseases. The adagium is that mitochondrial diseases can display any symptom, at any age and any time. In general, the course of mitochondrial diseases is progressive, causing substantial morbidity and mortality [10]. No effective treatment exists for the vast majority of mitochondrial diseases [4].

The clinical diagnosis of a mitochondrial disease is often difficult to establish and therefore a scoring system has been developed for children and adults. The mitochondrial disease criteria (MDC) for children are based on the presence of specific clinical signs and symptoms (maximum 4 points), neuroimaging and metabolic abnormalities (maximum 4 points), and histological abnormalities (maximum 4 points) [11]. Score 1: mitochondrial disorde unlikely; score 2-4: possible mitochondrial disorder; score 5-7: probable mitochondrial disorder; score 8-12: definite mitochondrial disorder. For adults the clinical diagnosis of mitochondrial disease is based on the modified Walker criteria for mitochondrial disease [12]. This scoring system consists of major and minor criteria, which results in a definite mitochondrial disease score ( 2 major, or 1 major +2 minor), or in a probable score ( 1 major +1 minor, or 3 minor), or in a possible score ( 1 major, or clinical +1 minor).

\subsubsection{Laboratory Assays}

In addition to an extensive clinical evaluation, also electron-microscopy, biochemical (ATP, lactate), and/or enzymological measurements can be performed to establish a diagnosis of a mitochondrial disease. The latter examination is usually performed on a muscle tissue biopsy, or in patient-derived cell lines (fibroblasts). A needle biopsy suffices, which is usually very welltolerated [6] and the enzymatic assay can be performed on fresh or frozen material. By using specific compounds, each enzyme complex can be tested individually and a deficiency determined. In case the enzyme deficiency is also present in fibroblast, this allows complementation assays to validate a genetic defect. 


\subsubsection{Genetic Diagnosis}

Due to the dual genetic origin of mitochondria, a causative genetic defect can be found in either the mtDNA or in a nuclear gene. In only very few cases, the clinical and biochemical criteria point directly to a gene, like in Leigh syndrome with complex IV deficiency, which is generally caused by mutations in the Surf 1 gene. Such a gene can be efficiently screened by Sanger sequencing. Sanger sequencing is also used to test relatives of patients with an established genetic defect. In the majority of less straightforward cases, as a first step, usually the entire mtDNA of $16,569 \mathrm{bp}$ is being analysed by next-generation sequencing (NGS). Sequencing is performed on a NGS platform using enrichment of a single entired long-range mtDNA PCR fragment as a library preparation step, these steps are according manufacture's instructions. The quantification accuracy of using NGS in mtDNA samples is determined by varying the threshold levels of different substitutions mutations, small indels, and large deletions. The levels of large deletions are confirmed by quantitative qPCR method. A similar qPCR technique is used to determine mtDNA depletion in tissue, were mtDNA copy number (MTND1) is normalised to the nuclearDNA copy number $(B 2 M)$. Since, each cell contains multiple copies of mtDNA and each cell can contain multiple mitochondria, a mutation only leads to disease, if the mutation load exceeds a tissue-specific threshold [13]. This combination of 2 types of mtDNA (wild-type and mutated mtDNA) is called heteroplasmy, whereas having accuracy one mtDNA type is called homoplasmy. The chance of identifying an mtDNA mutation is highest in the affected tissue, as differences in mutation load exist between tissues and a mutation can also disappear, for example, from blood. In the affected tissue the mutation load is usually highest and when using blood, the coverage has to be sufficiently high to detect low-mutation loads. It is also relevant to exclude involvement of the mtDNA as this has important consequences for the recurrence risk. In case, no causative genetic defect has been identified in the mtDNA, nuclear genes will be sequenced. This can be a targeted panel approach, only screening known mitochondrial (disease) genes, or an unbiased analysis of all genes (whole exome sequencing or whole genome sequencing). The latter approach allows the identification of novel genes as well, which may require functional validation to unambiguously link the gene defect to the disease. 


\subsubsection{Difficulty in Establishing a Genetic Diagnosis}

The clinical and dual genetic heterogeneity makes the diagnosis of mitochondrial diseases challenging. As extensive evaluation of clinical symptoms, biochemistry, immunohistochemistry, and quality and quantity of the mitochondria are not sufficient to pinpoint specifically a genetic cause and as 2 genomes are involved with possibly $>1500$ genes, it is evident that finding the genetic cause is a real odyssey. At the start of my thesis period, only $25 \%$ of the patients had a genetic diagnosis after extensive, costly and time-consuming sequential sequencing of individual candidate genes. Family studies and linkage analysis were fundamental to characterize unknown genes. However, the emergence of NGS has opened up a successful sequencing-based approach, allowing a more complete, more rapid and more successful diagnostic strategy.

\subsection{Transmission of Mitochondrial Disease}

Mitochondrial diseases have a dual genetic origin, which has implication for the transmission and recurrence risks. Nuclear gene defects may inherit in autosomal recessive or X-linked fashion or in autosomal dominant modus, but also de novo variants are quite frequent and should be considered. The mtDNA is transmitted by a mother to her children and during oogenesis a drastic decrease in mtDNA copy number occurs, which is followed by an amplification of this subset in maturing oocytes, the so-called mitochondrial genetic bottleneck. In carriers of a heteroplasmic mtDNA mutation, due to this bottleneck, the mtDNA mutation load can vary considerably among the oocytes of such a carrier and in the resulting offspring [13]. If a patient carries an mtDNA mutation as the cause of disease, 3 situations can apply with respect to recurrence. First, the mutation is de novo in the patient, which is the case for about $25 \%$ of patients with an mtDNA disease. The recurrence is low, as the mother does not carry the mutation, and prenatal diagnostics (PND) is offered for reassurance [14]. Second, the mother is carrier of a heteroplasmic mutation. In that case preimplantation genetic diagnostics (PGD) can be offered and unaffected embryos with a mutation load below the threshold of expression can be transferred [14, 15]. Finally, in case of a homoplasmic mutation or a very high mutation load in the mother, which makes it impossible or unlikely to select healthy embryos, mitochondrial replacement 
therapy (MRT) is the method of choice [16]. MRT is allowed in the UK for severe mtDNA disease cases, in which PGD is unlikely to be successful.

Despite the high impact the bottleneck has on the transmission of mtDNA disease, it is still poorly understood which mechanisms are involved. The size of the bottleneck is an issue of debate and if positive or negative selection occurs on mutations in addition to random genetic drift. Data on PGD embryos indicate that for some mutations only genetic drift applies, but for others this is complemented with positive (on mitochondrial membrane potential, m.8993T>G) or negative selection (on OXPHOS function, m.3243A>G) [18]. It is evident that insight in these mechanisms influences the reproductive choices and the chances of having unaffected offspring.

\section{Animal Models to Study the Transmission of mtDNA Variants and Disease}

A number of limitations prevent an accurate study of transmission of mtDNA disease in humans. The main issue is the availability of oocytes and embryos to study the transmission of heteroplasmic mtDNA variants [17]. Due to these limitations, family studies have been performed in order to get indirect information on the segregation of these variants $[18,19]$. Studies have reported random segregation and selection alike. Mouse models have been performed as well, both for fundamental investigations on the bottleneck as well as for limited studies on the transmission of mtDNA variants. Rodents have high numbers of offspring, but the number of strains carrying mtDNA mutations is still small [2]. A commonly used model for transmission is the 'mutator mouse' which is a mouse model with a proofreading-deficient mitochondrial DNA polymerase (PolgA) [20]. In particular, a critical Aspartate residue has been replaced with an Alanine residue of the second exonuclease domain (D257A). In the offspring, the mtDNA mutations are random and unevenly distributed and can accumulate clonally in somatic cells, causing a mosaic of respiratory chain deficiency in tissues such as heart, skeletal muscle and brain [20]. Moreover, the analysis of these tissues has shown that mtDNA mutator mice contain an increased load ( 3-5 times) of the somatic point mutations in the mtDNA and an increase in amount of mtDNA 
eliminated [20]. Eventually, the increase in somatic mtDNA mutations is associated with a reduction in the duration of life and early onset of aging-related phenotypes [20].

Zebrafish could be a good alternative model to study the mtDNA bottleneck, being cheap, easy to manipulate and producing large amounts of offspring. The mtDNA of zebrafish contains the same number of 37 evolutional genes as the human mtDNA of which also 13 are protein-encoding genes. Also, many nuclear structural OXPHOS, sub- and assembly units are evolutionary similar to human, which makes the zebrafish potentially an excellent model to study mitochondrial and mtDNA transmission. A first study on mtDNA copy number in oocytes, primordialgerm-cells and non-germ-cells in zebrafish has been performed by our group, which revealed that the genetic bottleneck is a well-conserved process between different species and ranging from fish to man [21]. During embryogenesis the genetic bottleneck showed a depth of mtDNA copy number $\sim 170$ mtDNA molecules per primordial-germ-cell and 50 mtDNA molecules in a non-germ-cell, which is in the range of what has been reported for mammals [21] and occurring at the same moment during development. Also, the large increase in mtDNA copy number during oogenesis is comparable validating further the relevance of using zebrafish for studying the human bottleneck. The zebrafish seems to be a highly suitable model organism for these studies, as it is possible to isolate at low costs large amounts of primordial-germ-cells, oocytes, and early somatic cells, to characterize the complete mitochondrial bottleneck in early embryonic development [21, 22] and study the factors underlying this bottleneck by manipulating the possible factors involved.

\section{Aims and Thesis Outline}

Mitochondrial diseases result from a reduced energy production capacity in the mitochondria due to deficiencies in the oxidative phosphorylation (OXPHOS) system. These are characterized by highly heterogeneous and tissue-specific clinical manifestations, typically involving tissues with high energy requirements, like brain, heart and muscle. As the OXPHOS system is under dual genetic control, mutations in both the mitochondrial DNA and nuclear DNA can lead to mitochondrial disease. Mainly as a consequence of the large genetic heterogeneity 
(possibly more than 1500 genes involved) and clinical variability, for the majority of patients a genetic cause was unknown at the start of the project. The first aim of this thesis was to identify and functionally characterize the underlying genetic cause in either the mtDNA or in nuclear genes in patients with OXPHOS disease and to prevent recurrence in the offspring. In case of nuclear gene mutations prenatal diagnosis or preimplantation diagnosis can be offered to prevent the recurrence of the mitochondrial disorder. However, in case of mtDNA disease this is more complex as the mitochondrial bottleneck defines how mtDNA mutations are being distributed. Therefore, the second aim of this thesis is to develop an animal model to study and manipulate the mitochondrial bottleneck. Zebrafish seems the best model to characterize the factors which define the transmission of mitochondria and the mitochondrial DNA. These 2 aims were split in the following objectives:

1) To demonstrate the impact of next-generation sequencing on genetic testing

2) To identify novel gene defects in patients with oxidative phosphorylation deficiency using next-generation sequencing and to characterize potentially disease-causing variants functionally in patient-derived or human cell lines

3) To develop a zebrafish model, in which the mitochondrial bottleneck can be manipulated

4) To study the effect of a low level of mtDNA copy-number on embryogenesis in zebrafish and characterize the underlying processes

In Chapter 2, we present next-generation sequencing technology and discuss the impact it has on identifying causative variants, which can lead to a genetic diagnosis in a number of genetic diseases. The potential and limitations of different technologies and diagnostic approaches (targeted NGS, whole-exome sequencing, RNA-Seq and whole genome sequencing) are being discussed for different diseases. As NGS can detect all almost variants in the exome or genome, we explored as a proof-of-concept, if genetic modifiers or risk factors can be detected, which can explain differences in clinical severity among carriers of the same gene defect. Putative genetic risk modifiers were identified in the cancer syndrome, Lynch syndrome, by testing a panel of 154 candidate genes (Chapter 3). A comparable approach can be applied to identify genetic modifiers in 
mitochondrial diseases. In Chapter $\mathbf{4}$ and $\mathbf{5}$ we applied whole-exome sequencing (WES) to identify the primary genetic cause in patients with mitochondrial diseases. The diagnosis was based on clinical symptoms, including MRI, and deficiencies in OXPHOS complexes in muscle and/or fibroblasts of the patients. Evidence for the pathogenicity or these novel variants in partly novel genes was provided by complementation assays. In 3 Moroccan families with Leigh syndrome we found the same genetic defect in a thiamine transporter gene (Chapter 4), resolving a new possible treatable cause of Leigh syndrome. In Chapter 5, we describe 3 families with paediatric, mitochondrial cardiomyopathy with early-onset brain disease and multiple OXPHOS deficiencies, having novel genetic defects and affected genes in mitochondrial-encoded translation proteins. In Chapter 6 we explore the role of a reduced mtDNA copy number by knocking down TFAM using morpholinos during early zebrafish development. The effect of a reduced mtDNA copy number on mitochondrial function and embryonic development was assessed and affected pathways were identified by transcriptomics. The general discussion (Chapter 7) discusses the impact of next-generation sequencing on the diagnostics of mitochondrial disease and the use of zebrafish for studying the mechanism of the genetic bottleneck. In Chapter 8, the valorisation chapter, we describe the rapid use of NGS, WES and WGS in the clinical practice, and the cases, in which identification of the gene-defect has enabled directly gene-based therapies. 


\section{References}

1. Mitochondria. Available from: https://www.nature.com/scitable/topicpage/mitochondria-14053590.

2. Lane, N. and W. Martin. The energetics of genome complexity. Nature 2010 Oct 21 [cited 467 7318]; 2010/10/22:[929-34]. Available from: https://www.ncbi.nlm.nih.gov/pubmed/20962839.

3. Gray, M.W., Mitochondrial evolution. Cold Spring Harb Perspect Biol, 2012. 4(9): p. a011403.

4. Gorman, G.S., et al., Mitochondrial diseases. Nat Rev Dis Primers, 2016. 2: p. 16080.

5. Gnaiger, E., Mitochondrial Pathways and Respiratory Control. 4th edition ed. 2014.

6. Koene, S.S., JAM,, Mitochondrial medicine, a clinical guideline. 2011.

7. Institute, B. Human MitoCarta2.0. 2019, 5 August; Available from: https://www.broadinstitute.org/files/shared/metabolism/mitocarta/huma n.mitocarta2.0.html.

8. Taylor, R.W. and D.M. Turnbull, Mitochondrial DNA mutations in human disease. Nat Rev Genet, 2005. 6(5): p. 389-402.

9. Skladal, D., J. Halliday, and D.R. Thorburn, Minimum birth prevalence of mitochondrial respiratory chain disorders in children. Brain, 2003. 126(Pt 8): p. 1905-12.

10. Mitochondrial Medicine Society's Committee on, D., et al., The in-depth evaluation of suspected mitochondrial disease. Mol Genet Metab, 2008. 94(1): p. 16-37.

11. Morava, E., et al., Mitochondrial disease criteria: diagnostic applications in children. Neurology, 2006. 67(10): p. 1823-6.

12. Bernier, F.P., et al., Diagnostic criteria for respiratory chain disorders in adults and children. Neurology, 2002. 59(9): p. 1406-11.

13. Otten, A.B. and H.J. Smeets, Evolutionary defined role of the mitochondrial DNA in fertility, disease and ageing. Hum Reprod Update, 2015. 21(5): p. 671-89.

14. Sallevelt, S., Dreesen JCFM, de Coo, IFM et al., Selecting the right embryo in mitochondrial disorders, ed. C. 17. Vol. Chapter 17:231-246. 2015: Molecular Genetics in Reproductive Medicine.

15. Sallevelt, S.C.E.H., et al., Preimplantation genetic diagnosis in mitochondrial DNA disorders: challenge and success. Journal of Medical Genetics, 2013. 50(2): p. 125-132.

16. Foundation, U.M.D., Mitochondrial Replacement Therapy. 2019, 5 August.

17. Keeling, P.J., et al., The tree of eukaryotes. Trends Ecol Evol, 2005. 20(12): p. 670-6.

18. Otten, A.B.C., et al., Mutation-specific effects in germline transmission of pathogenic mtDNA variants. Hum Reprod, 2018. 33(7): p. 1331-1341. 
19. Gabaldon, T. and M.A. Huynen, Reconstruction of the proto-mitochondrial metabolism. Science, 2003. 301(5633): p. 609.

20. Trifunovic, A., et al., Premature ageing in mice expressing defective mitochondrial DNA polymerase. Nature, 2004. 429(6990): p. 417-23.

21. Otten, A.B., et al., Differences in Strength and Timing of the mtDNA Bottleneck between Zebrafish Germline and Non-germline Cells. Cell Rep, 2016. 16(3): p. 622-30.

22. Otten, A.B., et al., Replication Errors Made During Oogenesis Lead to Detectable De Novo mtDNA Mutations in Zebrafish Oocytes with a Low mtDNA Copy Number. Genetics, 2016. 204(4): p. 1423-1431. 



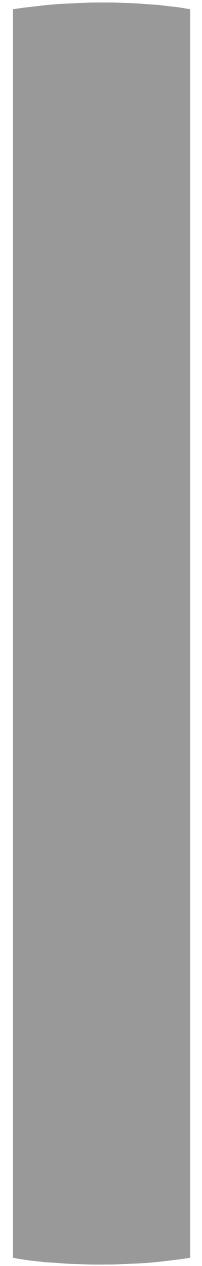




\section{Part I}

Whole Exome Sequencing a

Successfull Strategy to Identify Genetic

Defects in Mitochondrial Disease 
CHAPTER 2 


\section{Next-Generation Sequencing in Genetic Diagnosis, Risk Prediction and Disease Classification}

Based on:

First: Part of the Publication: Kamps $R^{*}$, Brandão RD*, Bosch BJ, Paulussen AD, Xanthoulea S, Blok MJ, Romano A.

Int J Mol Sci 2017 Jan31;18 (2).pii:doi: 10.3390/ijms18020308,

https://www.mdpi.com/1422-0067/18/2/308 (Next-Generation Sequencing in Oncology: Genetic Diagnosis, Risk Prediction and Cancer Classification). * these authors share first authorship

Second: Part of the Publication: Theunissen TEJ, Nguyen M, Kamps R, Hendrickx AT, Sallevelt SCEH, Gottschalk RWH, Calis CM, Stassen APM, de Koning B, Mulder-Den Hartog ENM, Schoonderwoerd K, Fuchs SA, Hilhorst-Hofstee Y, de Visser M, Vanoevelen J, Szklarczyk R, Gerards M, de Coo IFM, Hellebrekers DMEI, Smeets HJM.

Front Genet. https://doi.org/10.3389/fgene.2018.00400 eCollection 2018 (Whole exome sequencing is the preferred, first strategy to identify known and novel causes of mitochondrial disease). 


\section{Introduction}

Next-generation sequencing (NGS), also called massive parallel sequencing, has been developed in the last 2 decades and allows simultaneous sequencing of millions of DNA fragments without previous sequence knowledge. This advanced technology has been a revolution compared to traditional sequencing methods, in which one or a few relatively short fragments of DNA, previously amplified by Polymerase Chain Reaction (PCR), could be sequenced per reaction. Due to the high costs and intensive work required, traditional sequencing was only performed on specific DNA regions or genes and for specific samples. For instance, genetic screening for mutations, as in breast/ovarian cancer or Lynch syndrome, was not long ago based on first screening of PCR fragments for DNA hetero-duplexes, which indicated the presence of a DNA variant. Only the PCR fragments with such heteroduplexes would then be sequenced. The limited throughput can be exemplified by the duration of the Human Genome Project, which launched in 1990, required 13 years and billions of dollars to sequence the complete human genome. With NGS, today a complete genome can currently be sequenced in a few days for less than $\$ 1000$ per genome. The implications and the impact of NGS in characterizing genetic disorders, in understanding the biological processes of diseases like cancer and in personalising patient care are unprecedented. In this chapter, the major milestones in NGS technology and the technical developments and application of NGS in the field of diagnostics, classification, therapeutics, and pharmacogenetics will be briefly addressed.

\section{NGS-Next-Generation Sequencing Technology}

The Sanger DNA sequencing method, also named chain terminator sequencing, was developed in 1977. This method, which was later automated and modified, was the gold standard for sequencing until the late 2000's [1]. In the first decade of this century, and number of different approaches for sequencing were developed, which evolved into commercially available NGS DNA sequencers. The first commercial NGS sequencer, based on pyrosequencing technology, was developed in 2004 by $454^{\circledR}$ and further commercialized by Roche. Sequencing by synthesis (SBS) technology was developed by Solexa and further commercialized by 
Illumina. Since then, speed and costs of NGS have decreased continuously, with a massive decline during the last eight years, and several NGS sequencers with different chemistries have been launched into the market (Figure 1).
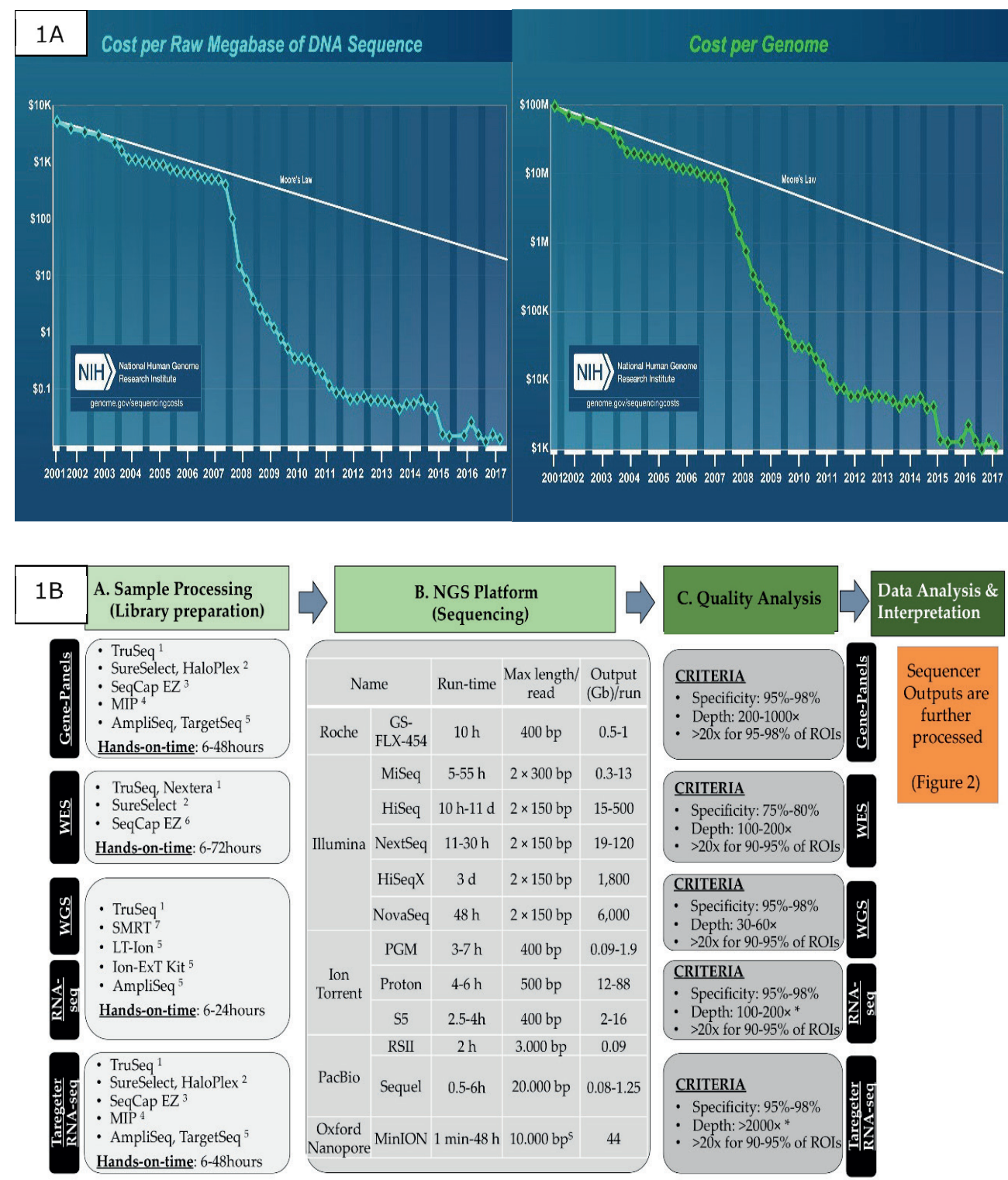

Figure 1. 1A) The Nationals Institutes of Health (NIH) reported in 2017 costs per raw megabase of DNA sequence and costs per genome. 1B) The pipeline illustrating the four major blocks in next-generation sequencing (NGS) studies. Illumina ${ }^{\circledR}{ }^{1}$; Agilent Technology ${ }^{\circledR} 2$; Nimblegen ${ }^{\circledR 3}$; MIP: Molecular Inversion Probe ${ }^{4}$, used to amplify in 
large numbers short specific regions of DNA (SciTools ${ }^{\circledR}$, Integrated DNA Technologies, Coralville, Iowa, U.S.); ThermoFisher ${ }^{\circledR 5}$; Roche ${ }^{\circledR 6}$; PacBio ${ }^{\circledR} 7$. Because of their recent development, information about the Qiagen GeneReader ${ }^{\circledR}$ and 10x Genomics $^{\circledR}$ technology are not included in this figure. \$ Users have reported up to $200,000 \mathrm{pb}$; * To detect low expressed transcripts, $>2000 \times$ coverage is needed.

Advantages of NGS sequencers are the high-throughput sequencing capacity of large or small genomic regions for many samples in parallel and the fact that they do not require previous knowledge of the genome per se. Nowadays, the use of NGS almost replaced conventional Sanger sequencing and is a very versatile approach for several clinical and non-clinical applications.

\subsection{NGS Technology, Historical Perspective and State-of-the-Art}

A thorough overview of the different NGS platforms and approaches (e.g., Illumina (Solexa) ${ }^{\circledR}$, Oxford Nanopores ${ }^{\circledR}$, PacBio $^{\circledR}$ or Roche ${ }^{\circledR}$ is available at the "Next-GenField-Guide" by Travis Glenn [2]. This synopsis was created in 2011, was last adapted in 2016, covering advantages, disadvantages, costs and overall performances of the different platforms. Current NGS technology can be divided in two major types, i.e., short- and long-read sequencing. For short-read sequencing Illumina ${ }^{\circledR}$ is market leader with cheap "sequencing by synthesis" (SBS) of reads shorter than 300 bp [3]. The ion semiconductor method (Ion Torrent ${ }^{\circledR}$ ) is another cheap short-read sequencer [4]. For long-read sequencing PacBio ${ }^{\circledR}$ is market leader, involving more expensive "single molecule real-time" (SMRT) technology with half of the data in the reads being $>50 \mathrm{~Kb}[5,6]$. The Oxford Nanopore Technologies ${ }^{\circledR}$ MinION, using single stranded pore technology, also allows to sequence very long molecules ( $>10 \mathrm{~Kb}$ ) [7] and at a relatively low cost, but with a relatively higher error rate compared to PacBio.

Short-read sequencing has the lowest costs per Gb and a very high accuracy (low final error-rate; $0.1 \mathrm{~Kb}$ ), and has become the method most frequently used today. In contrast, long-read sequencing has a lower accuracy (high final error-rate; $>1$ $\mathrm{Kb}$ ) and a higher costs per Gb. Nevertheless, long-read sequencing presents unique advantages for a number of applications. It improves the alignment in the 
bioinformatics pipeline (e.g., de novo assembly). It is also more suitable for familial haplotyping (allele phasing), for the detection of DNA and chromosomal structural variations, large chromosomal rearrangements, translocations, and repeat sequence and for the characterization of transcript-isoforms from RNA-Seq data, allowing the detection and frequency of alternative splice-events. Qiagen GeneReader $^{\circledR}$ [8], 10x Genomics ${ }^{\circledR}$ technology [9] and Illumina NovaSeq ${ }^{\circledR}$ sequencer [3] represent the latest developments in NGS. Qiagen GeneReader ${ }^{\circledR}$ is a versatile system that provides the complete NGS pipeline from library preparation by PCR-based targeted exon enrichment to data analysis (see next paragraph) but can be coupled to other technologies and sequencers as well. The 10x Genomics $^{\circledR}$ technology coupled with Chromium System [9] facilitates the preparation of libraries suitable for multiple NGS platforms, linking reads into extra-long (synthetic) reads for analysis.

\subsection{NGS Strategies}

Different NGS approaches can be used according to the questions to be addressed. The initial input material can be genomic DNA (DNA-Seq), messenger or noncoding RNA (RNA-Seq) or any nucleic/ribonucleic material obtained after specific procedures. It can be derived from large number of cells or even from single cells. The implementation of NGS technology can be visualised as four major blocks (Figure 1B).

(A.) Libray preparation or sample processing. The material is first fragmented mechanically or enzymatically to yield fragments whose sizes are compatible with the sequencing technology used (small fragments of 200-300 nucleotides for short-read sequencing, longer for the long-read sequencing). This material can be enriched to analyse a limited number of genetic regions (e.g., disease genepanels or microbes [10]) or all coding exons of the human genome (approximately 21,000 genes, Whole-Exome-Sequencing, WES). These regions are called regions of interest (ROIs). The complete genomic DNA can also be sequenced (Whole-Genome-Sequencing, WGS), which does not require an enrichment step (see Section 2.2.3). An amplification step through PCR with 4-12 cycles is perfomed in 
most cases. During this step, proper linkers and barcodes, which are unique nucleotide tags (6-8 $\mathrm{nt})$, are attached to the DNA fragments, which are necessary to link the final sequence to a specific sample or individual. DNA barcodes allow pooling of samples together in one single flowcell for the sequencing reaction.

(B.) Sequencing. Most common sequencers have been extensively described [2-7] and Figure 1B summarises a few characteristics that distinguish them. A review on the different sequencing chemistries can be found elsewhere [11].

(C.) Initial quality and raw data analyses. General read quality is assessed with FastQC [12]. Many pre-processing tools are available for removal of bad quality reads, trimming, etc. After mapping, specificity is determined, i.e., the fraction (\%) of the total number of predefined ROIs, which are correctly enriched and sequenced.

(D.) Variant calling and data interpretation. This last step is dependent on the specific application. In this chapter, some methods and bioinformatics tools relevant to data interpretation will be presented.

As the focus of the NGS work in this thesis is on detecting pathological variants in genes, I will concentrate on strategies to identify these variants. 


\subsubsection{Gene-Panels}

For clinical applications, the use of gene-panels to sequence only a limited number of genes of interest has been the first method of choice, because of its costefficiency, its high coverage of the ROIs and its simplicity in the data analyses [13]. It also prevents the identification of unsolicited findings, but, as a drawback, it will not allow the identification of novel genes. When the number of genes sequenced is restricted to the few already analysed in previous diagnostic tests using traditional methods, this is normally called targeted re-sequencing.

Different protocols are available to design and capture panels of genes and other ROIs. In most cases, companies providing library preparation kits, offer online tools to design the hybridisation probes or the PCR oligos to enrich the desired ROIs. Enrichment can be obtained via solid phase hybridisation, in-solution hybridisation (most frequently used) or PCR-based enrichment and can be followed by amplification via multiplex PCR, rolling-circle amplification (HaloPlex ${ }^{\circledR}$ ) or amplicon-based-microdroplet-PCR (RainDance ${ }^{\circledR}$ technology) [14]. The latter presents the advantage of simultaneously amplifying a large number of targeted regions into separate micro drops, thus keeping each amplification separate from the others and limiting the disturbance due to primer pair interactions. A cheap and flexible method to capture small regions of the genome for NGS analyses is the Molecular Inversion Probe (MIP) [15].

After sequencing, the analysis of raw data is relatively simple [13]. Due to the high coverage per nucleotide, the specificity is high and only particular DNA regions may be captured inefficiently (due to high GC nucleotide content, for instance). Normally, sequencing depth of around $80 \times$ is sufficient to detect germline variants that are present in a homo- or heterozygous status. In case of somatic mutations, a higher coverage is required $(>500)$ since mutations are usually present at low levels. 


\subsubsection{WES-Whole-Exome-Sequencing}

Protocols/kits to enrich the library for all exons are available from several companies and use the same or similar technologies as mentioned for the enrichment of gene-panels. Following sequencing, raw data analysis is performed to determine the quality of the sequencing data, checking for issues that may have occurred at the level of library preparation and/or sequencing. Both steps are crucial to obtain good quality data. A high sequence-on-target yield of more than $90 \%$ of the ROIs and coverage higher than $20 \times$ per nucleotide is necessary for sufficient specificity and sensitivity in heterozygous mutation detection. Normally, when less than $90 \%$ of the ROIs are sequenced but coverage is high, sample processing was suboptimal; when the ROIs are sufficiently sequenced $(>90 \%$ ) but coverage is low, then the sequencing reaction was suboptimal and re-sequencing is required $[16,17]$.

\subsubsection{WGS-Whole-Genome-Sequencing}

The holy grail of NGS is obviously whole genome sequencing in order to identify all variants present. WGS, or RNA-Seq (see below), are logical follow-up steps, in case WES did not reveal a causative variant in families with a high probability of carrying a genetic mutation. The major technical advantage of WGS is that the library preparation does not require any enrichment or amplification and the specificity is theoretically $100 \%$ (achieving around $95 \%-98 \%$ in practice) with a uniform coverage throughout the input material. Therefore, the possibility to miss a disease-causing variant (or any other information) for technical reasons such as inefficient probe targeted enrichment, inefficient amplification of a specific ROI or PCR amplification artefacts do not apply to WGS [18-20]. However, not a single technique can detect all variants in the entire genome and for true complete genome analysis, hybrid sequencing using both short-read and long-read sequencing is required.

The most important obstacles in applying WGS on a routine scale are the high costs, the complex pipeline for data analyses and data interpretation. However, the more the costs of NGS decrease, bioinformatics tools improve and our understanding of the function of the non-coding part of the genome increases 
(also via the development of novel functional assays), the more this approach will be implemented. It is foreseeable that in the near future WGS will be performed routinely for every diagnostic question as a generic test and ROIs will be subsequently selected ad-hoc in silico for each specific application from the raw data, in order to find the causative genetic variants [21]. As WGS enables to detect chromosomal abnormalities and pathogenic intronic variants it increases the number of diagnoses in selected cohorts to $10-35 \%[20,21]$.

\subsubsection{Identification of Disease-Causing Variants using RNA-Seq or Transcriptomics}

Splicing, the process of removal of the introns in the pre-mRNA molecule, is highly regulated by the RNA-splicing machinery and depends on specific genetic sequences to mark intron/exon junctions. Additionally, epigenetic factors (chromatin conformation and histone modifications) have also been implicated in the regulation of splicing [22]. Incorrect splicing, which occurs due to the presence of genetic variants, mostly in intronic regions, has been implicated as a cause of genetic disease, including hereditary cancer syndromes [23-28]. Targetedsequencing or WES analysis is mainly limited to the protein-coding region (exons) of the genome and to a few intronic nucleotides, which are the most conserved splice sites adjacent to the exon, and do not cover the remaining of the introns, promoter regions and non-coding RNAs, which are important for gene-expression regulation. In addition, computer-based tools and pipeline analyses are less accurate in predicting the effect of DNA-variants on gene-expression or RNA splicing. Therefore, mutations with a potentially pathogenic effect on RNA expression or processing are either not included in the regions of interest during WES sequencing or are missed by bioinformatics analysis [29-33]. Sequencing the transcriptome by RNA-Seq is therefore a valuable approach to detect variants affecting RNA amounts, including mutations in transcription binding sites or promotors, or splicing in tissues where the gene is expressed. RNA-Seq allows the analysis of the transcriptome at an unprecedented depth [34-37]. Similar to WGS and unlike Sanger sequencing, it does not depend on previously known sequences, which is of particular relevance when studying the mRNA and its alternative splicing events. RNA-Seq can be performed with short-read and long read, single molecule sequencers, with the long-read sequencers having the advantage to sequence entire RNA molecules covering all variants and splice events (described 
in Section 2). Combining RNA-Seq with WGS protocols and integrated analyses will also aid identification of RNA-editing events and will increase the chance of finding the causative DNA variant. RNA-Seq also has some limitations. RNA is less stable than DNA and requires higher care in handling and storage of the samples. In addition, RNA expression (and processing) is tissue-dependent and the tissue available for analysis may not express the gene with the defect. A number of studies has shown the benefit of RNA-Seq in screening splice variants in inherited diseases so far. Recently, a publication reported the success of transcriptome sequencing to increase mutation-detection rate in undiagnosed mitochondrial disorders [38]. This resulted in the identification of causative, non-exonic regulatory variants in an additional $10 \%$ of patients. One should realize that by RNA-Seq only the genes are analysed, which are expressed in the tissue of investigation, which might affect the success rate for specific diseases. For example, in fibroblasts 2574 (68\%) of the 3768 known disease genes reported in OMIM are expressed, which include most of the mitochondrial genes. Currently, the diagnostic WES -yield varies from $25-50 \%$ for rare muscular disorders [39], and $39 \%-69 \%$ for mitochondrial disorders $[38,40]$. In the 2 papers described RNA-Seq-yield adds a $35 \%$ for rare muscular disorders [39], and $10 \%$ for mitochondrial disorders [38].

\subsection{Data Analyses and Interpretation}

After raw data analysis has demonstrated sufficient quality, data analyses and interpretation needs different pipelines depending on the approach (gene-panel, WES, WGS or RNA-Seq) and on the questions to be answered. Figure 2 illustrates briefly the major steps for DNA-Seq used in genetic diagnostics to interpret data obtained from an Illumina ${ }^{\circledR}$ platform, but which is comparable to other platforms used. 


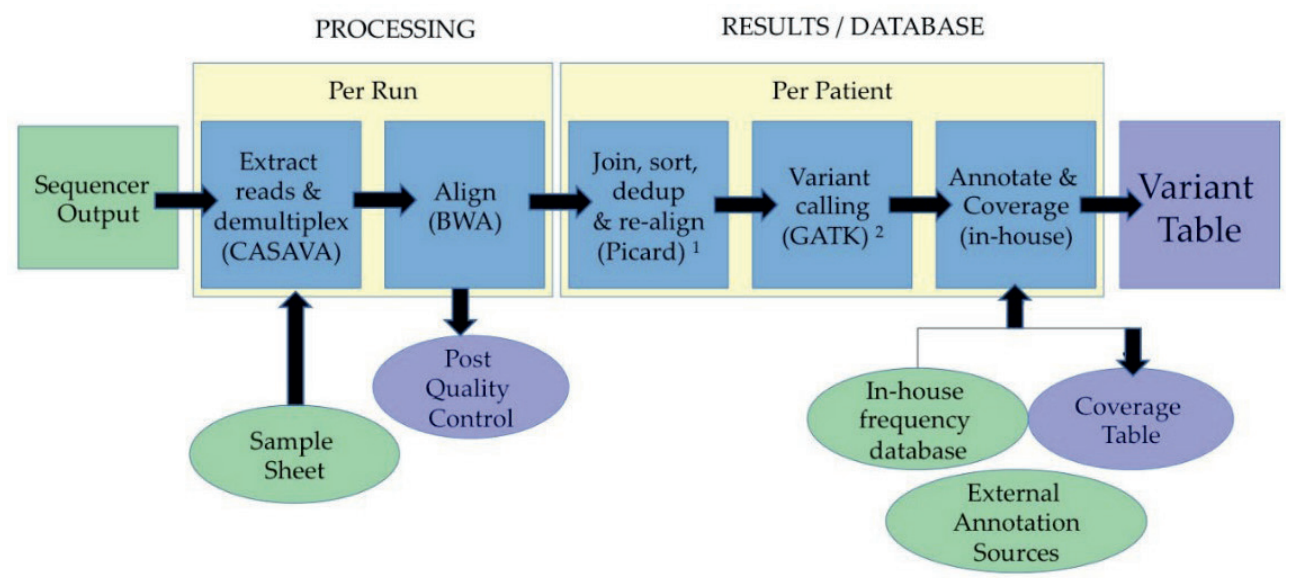

Figure 2. Data interpretation pipeline. Example of DNA-Seq Bioinformatics Pipeline for Illumina ${ }^{\circledR} .{ }^{1}$ This step removes duplicate sequences using the Picard program; ${ }^{2}$ GATK: Genome Analysis ToolKit.

Base-calling is performed using software like the Casava pipeline that produces Fast-Q files (raw-initial data), which can then be aligned to the human reference genome using Burrows-Wheeler-Alignment tool (BWA). Single base variants can be identified using Sequence-Alignment-MAP tools (SAM) and annotated. Additional software and scripts match the data from NGS analysis to variants in reference databases (RefGene, dbSNP and UCSC genome browser [21]), in order to identify variants already annotated and identify new variants present in the NGS sample. This ultimately generates the "variant table" (Figure 2) that contains a list of all identified variants with, if available, information compiled from other databases such as allele frequency, pathogenicity scores, conservation and literature references.

For identifying the genetic cause of a disease in a patient or in a family, the goal is to assign clinical significance to the variants identified. Several databases (OMIM [41], LOVD [42], ClinVar [43]), catalogue known variants with respect to their pathogenicity, but for unknown variants pathogenicity has to be determined. A variety of tools is available that can be used to predict their effect at the protein level, protein activity, RNA splicing and ultimately help assess the presence of protein damaging alterations (Alamut, Polyphen, Sift). This analytical process can be performed manually for each variant or can be automated. Several guidelines are available to assist such classification [44]. The ultimate proof of the 
pathogenicity of a variant may be functional characterization (or any other genetic diseases), requiring specific functional assays (in vitro, in vivo) and/or cosegregation studies of the variant with the disease among relatives. Similar considerations and sorts of analyses apply to studying and deciphering the effects of somatic mutations occurring in cancer.

\subsection{Conclusions}

Different methods and technologies are currently available to identify the genetic cause of diseases, with NGS becoming the prime diagnostic test. NGS has brought unprecedented advances in understanding the biology of diseases, with important clinical implications. The costs of short-read sequencing have become extremely low. Nowadays, WES/WGS (yield 50-60\%) and RNA-Seq (yield 35-10\%) are becoming routine in clinical practice for heterogeneous genetic disorders, but the eventual step in diagnostics in the years to come will be WGS/RNA-Seq by shortread sequencing and later by hybrid sequencing, involving long-read sequencing for structural variants and repeat sequences. Technical and bioinformatical advances make NGS technology increasingly more powerful. It is crucial that these progresses are accompanied by increasing awareness of its strong potential by physicians and patients. It is also of fundamental importance that the progress is paralleled by strict monitoring the use of these technologies in relation to ethical issues, especially as NGS will in future not only be performed to identify the cause of disease, but also in the form of the personal genome to guide the life of a healthy person. Unfortunately, despite all significant improvements in the diagnosis of genetic diseases, the knowledge of the genetic cause has only limitedly led to the development of novel, effective therapies, which will be the challenge for the years to come. 


\section{References}

1. Marziali, A. and M. Akeson, New DNA sequencing methods. Annu Rev Biomed Eng, 2001. 3: p. 195-223.

2. Next-Gen-Field-Guide. http://www.molecularecologist.com/next-genfieldguide-2014. accessed on 9th of January 2017].

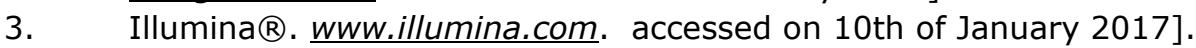

4. Ion-Torrent $\circledast$. http://www.thermofisher.com. accessed on 7th of November 2016].

5. PacBio $®$. http://www.pacb.com/ accessed on 11th of November 2016].

6. Roche $\AA$. http://www. roche.com/. accessed on 11th of November 2016].

7. Nanopore $\circledR$, O. https://www.nanoporetech.com/. accessed on 11th of November 2016].

8. GeneReader. https://www.qiagen.com/nl/resources/technologies/ngs/. accessed on 10th of January 2017].

9. 10X-Genomics $®$. http://www.10xgenomics.com/technology/. accessed on 10th of December 2016].

10. Duncavage, E.J., et al., Hybrid Capture and Next-Generation Sequencing Identify Viral Integration Sites from Formalin-Fixed, Paraffin-Embedded Tissue. The Journal of Molecular Diagnostics : JMD, 2011. 13(3): p. 325333.

11. Goodwin, S., J.D. McPherson, and W.R. McCombie, Coming of age: ten years of next-generation sequencing technologies. Nat Rev Genet, 2016. 17(6): p. 333-351.

12. Andrews, S. FastQC: a quality control tool for high-throughput sequence data. Available from: http://www.bioinformatics.babraham.ac.uk/projects/fastqc/.

13. Jori, B., et al., Germ-line variants identified by next generation sequencing in a panel of estrogen and cancer associated genes correlate with poor clinical outcome in Lynch syndrome patients. Oncotarget, 2015. 6(38): p. 41108-22.

14. RainDance $\Re$. http://www.raindancetech.com/. accessed on 11 th of November 2016].

15. Niedzicka, M., et al., Molecular Inversion Probes for targeted resequencing in non-model organisms. Sci Rep, 2016. 6: p. 24051.

16. Bao, R., et al., Review of current methods, applications, and data management for the bioinformatics analysis of whole exome sequencing. Cancer Inform, 2014. 13(Suppl 2): p. 67-82.

17. Leggett, R.M., et al., Sequencing quality assessment tools to enable datadriven informatics for high throughput genomics. Front Genet, 2013. 4: p. 288.

18. Klinische Genetica. Gene-panel Mitochondria, . 2019, 2 August [cited 2019 2 August]; Available from: https://www.labmaastricht.nl/klinischegenetica.

19. Ekblom, R. and J.B. Wolf, A field guide to whole-genome sequencing, assembly and annotation. Evol Appl, 2014. 7(9): p. 1026-42. 
20. Gonzaga-Jauregui, C., J.R. Lupski, and R.A. Gibbs, Human genome sequencing in health and disease. Annu Rev Med, 2012. 63: p. 35-61.

21. Kent, W.J., et al., The human genome browser at UCSC. Genome Res, 2002. 12(6): p. 996-1006.

22. Brandão, R.D., et al., Characterisation of unclassified variants in the $B R C A 1 / 2$ genes with a putative effect on splicing. Breast cancer research and treatment, 2011. 129(3): p. 971-82.

23. Anczuków, O., et al., Unclassified variants identified in BRCA1 exon 11: Consequences on splicing. Genes Chromosomes Cancer, 2008. 47(5): p. 418-426.

24. Bonnet, C., et al., Screening BRCA1 and BRCA2 unclassified variants for splicing mutations using reverse transcription PCR on patient RNA and an ex vivo assay based on a splicing reporter minigene. J Med Genet, 2008. 45(7): p. 438-446.

25. Campos, B., et al., RNA analysis of eight BRCA1 and BRCA2 unclassified variants identified in breast/ovarian cancer families from Spain. Hum Mutat, 2003. 22(4): p. 337.

26. Caux-Moncoutier, V., et al., Impact of BRCA1 and BRCA2 variants on splicing: clues from an allelic imbalance study. Eur J Hum Genet, 2009. 17(11): p. 1471-1480.

27. Fackenthal, J.D., et al., BRCA2 T2722R is a deleterious allele that causes exon skipping. Am J Hum Genet, 2002. 71(3): p. 625-631.

28. Castera, L., et al., Next-generation sequencing for the diagnosis of hereditary breast and ovarian cancer using genomic capture targeting multiple candidate genes. Eur J Hum Genet, 2014. 22(11): p. 1305-13.

29. Cragun, D., et al., Panel-based testing for inherited colorectal cancer: a descriptive study of clinical testing performed by a US laboratory. Clin Genet, 2014. 86(6): p. 510-20.

30. Yurgelun, M.B., et al., Identification of a Variety of Mutations in Cancer Predisposition Genes in Patients With Suspected Lynch Syndrome. Gastroenterology, 2015. 149(3): p. 604-13 e20.

31. Lincoln, S.E., et al., A Systematic Comparison of Traditional and Multigene Panel Testing for Hereditary Breast and Ovarian Cancer Genes in More Than 1000 Patients. J Mol Diagn, 2015. 17(5): p. 533-44.

32. Frey, M.K., et al., Rescreening for genetic mutations using multi-gene panel testing in patients who previously underwent non-informative genetic screening. Gynecol Oncol, 2015. 139(2): p. 211-5.

33. Cloonan, N., et al., Stem cell transcriptome profiling via massive-scale mRNA sequencing. Nat Meth, 2008. 5(7): p. 613-619.

34. Mortazavi, A., et al., Mapping and quantifying mammalian transcriptomes by RNA-Seq. Nat Meth, 2008. 5(7): p. 621-628.

35. Sultan, M., et al., A Global View of Gene Activity and Alternative Splicing by Deep Sequencing of the Human Transcriptome. Science, 2008. 321(5891): p. 956-960. 
36. Morin, R.D., et al., Profiling the HeLa S3 transcriptome using randomly primed CDNA and massively parallel short-read sequencing. BioTechniques, 2008. 45(1): p. 81-94.

37. Ramaswami, G., et al., Accurate identification of human Alu and non-Alu RNA editing sites. Nat Methods, 2012. 9(6): p. 579-81.

38. Kremer, L.S., S.B. Wortmann, and H. Prokisch, "Transcriptomics": molecular diagnosis of inborn errors of metabolism via RNA-sequencing. J Inherit Metab Dis, 2018. 41(3): p. 525-532.

39. Cummings, B.B., et al., Improving genetic diagnosis in Mendelian disease with transcriptome sequencing. bioRxiv, 2016.

40. Theunissen, T.E.J., et al., Whole Exome Sequencing Is the Preferred Strategy to Identify the Genetic Defect in Patients With a Probable or Possible Mitochondrial Cause. Front Genet, 2018. 9: p. 400.

41. OMIM. http://omim/org/ accessed on 10th December 2016].

42. Fokkema, I.F., et al., LOVD v.2.0: the next generation in gene variant

43. Clinvar. ClinVar. 2019, 6 August; Available from: https://www.ncbi.nlm.nih.gov/clinvar/.

44. Wallis, Y.P., S; McAnulty, C; Bodmer, D; Sister-man, E; Robertson, K; Moore, D; Abbs, S; Deans, Z; Devereau, A., Practice Guidelines for the Evaluation of Pathogenicity and the Reporting of Sequence Variants in Clinical Molecular Genetics. 2013, Birmingham, UK: Association for Clinical Genetic Science.

The Supplementary Material for the review Int. J. Mol. Sci. 2017, 18(2), 308; https://doi.org/10.3390/ijms18020308

The Supplementary Material for the second WES article can be found online at: https://www.frontiersin.org/articles/10.3389/fgene.2018.00400/full\#supple mentary-material 
CHAPTER 3

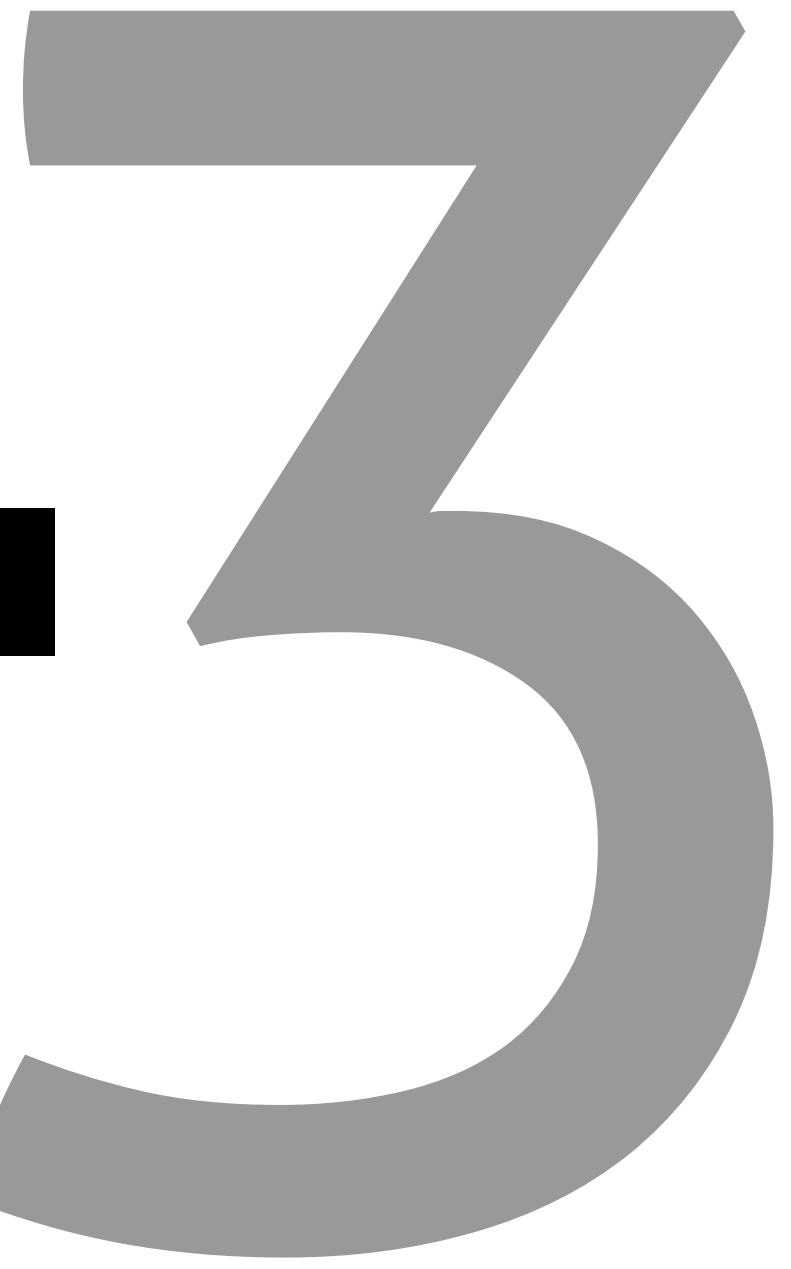




\section{Germ-line Variants Identified by Next-Generation Sequencing in a Panel of Estrogen and Cancer Associated Genes Correlate with Poor Clinical Outcome in Lynch Syndrome Patients}

Balazs Jóri, Rick Kamps*, Sofia Xanthoulea*, Bert Delvoux, Marinus J. Blok, Koen K. Van de Vijver, Bart de Koning, Felicia Trups Oei, Carli M. Tops, Ernst J. M. Speel, Roy F. Kruitwagen, Encarna B. Gomez-Garcia, Andrea Romano

*These authors have contributed equally to this work 


\section{Abstract}

Background: The risk to develop colorectal and endometrial cancers among subjects testing positive for a pathogenic Lynch syndrome mutation varies, making the risk prediction difficult. Genetic risk modifiers alter the risk conferred by inherited Lynch syndrome mutations, and their identification can improve genetic counseling. We aimed at identifying rare genetic modifiers of the risk of Lynch syndrome endometrial cancer.

Methods: A family based approach was used to assess the presence of genetic risk modifiers among 35 Lynch syndrome mutation carriers having either a poor clinical phenotype (early age of endometrial cancer diagnosis or multiple cancers) or a neutral clinical phenotype. Putative genetic risk modifiers were identified by Next Generation Sequencing among a panel of 154 genes involved in endometrial physiology and carcinogenesis.

Results: A simple pipeline, based on an allele frequency lower than 0.001 and on predicted non-conservative amino-acid substitutions returned 54 variants that were considered putative risk modifiers. The presence of two or more risk modifying variants in women carrying a pathogenic Lynch syndrome mutation was associated with a poor clinical phenotype.

Conclusion: A gene-panel is proposed that comprehends genes that can carry variants with putative modifying effects on the risk of Lynch syndrome endometrial cancer. Validation in further studies is warranted before considering the possible use of this tool in genetic counseling. 


\section{Introduction}

Lynch Syndrome is caused by mutations in one out of four mismatch repair (MMR) genes - MLH1, MSH2, MSH6 and PMS2 - and results in a $25 \%$ to $75 \%$ lifetime risk of colorectal cancer and $60 \%$ risk of endometrial cancer in women [1].

Genetic testing helps estimating the individual risk, plan appropriate care, screening and prophylactic treatments. Nevertheless, the risk to develop cancer conferred by a MMR mutation is modified by both the environment and genetic risk modifiers. The identification of such genetic risk modifiers can improve risk prediction and genetic counseling, through the individualization of surveillance programs and the evaluation of benefits versus burdens associated with prophylactic strategies $[1,2]$.

Searching for genetic risk modifiers is a challenge due to their expected small effect-size and their non-pathogenicity in the absence of a pathogenic mutation [2-4]. Genome-wide-association studies have identified loci and single nucleotide polymorphisms (SNPs) with risk modifying effects [5-8]. More recently, Next Generation Sequencing (NGS) of the whole genome or combined with gene-panels is emerging in genetic diagnostic to detect germ-line variants predisposing to cancer $[4,9-11]$.

Here, NGS combined with a 154 gene-panel was used to identify rare variants (minor allele frequency, MAF, <0.001) acting as gene-modifiers of Lynch syndrome MMR mutations. Unlike cancer somatic mutations, which map exclusively on tumor suppressors and oncogenes, the carcinogenic effect of germline variants can be indirect $[2,4,11]$, hence they can map within but also outside the genes classically associated with cancer. A defect in the genome stability conferred by a Lynch syndrome MMR mutation can be aggravated by disturbed tissue homeostasis. Therefore, as proof of principle for Lynch syndrome related endometrial cancer, a panel that included genes controlling the endometrial physiology and homeostasis (i.e. hormone signaling) beside those associated with cancer was complied. A family based approach was used: the presence of candidate genetic risk modifiers was evaluated among 35 patients carrying 
already a MMR mutation and characterized by either a poor clinical phenotype (early age of diagnosis or the diagnosis of multiple cancers) or by a neutral clinical outcome (diagnosis with endometrial cancer only and late in life).

\section{Results}

\section{Patients}

Table 1 shows the clinical features of the women enrolled. All were diagnosed with endometrial cancer between 31-81 years (mean: $53.1 \pm 10.7$ ). Subjects belonged to 29 families with a Lynch syndrome mismatch repair (MMR) pathogenic mutation, and all women carried the mutation. These mutations will be referred to as 'familial MMR mutations' (Table 1). One familial MMR mutation in MSH6 (c.3729_3732dupATTA - p.(Phe1245Ilefs*31)) was a founder mutation common to nine subjects belonging to six families.

Eleven women were diagnosed with a second Lynch syndrome tumor (two in the breast, six in the intestine, three in the ovaries). One woman developed colorectal and urinary tract cancers and one was diagnosed twice with colorectal cancer (14 years apart). Additionally, 14 women developed endometrial cancer at the age of 49 or earlier. In the following analyses, women diagnosed with more than one cancer, or with endometrial cancer before 50 (49 or younger) are defined as patients with poor clinical phenotypes (or outcome, $n=23$ ), whereas 14 patients who developed only endometrial cancer and after the age of 50 are defined as having a neutral clinical phenotype. 


\begin{tabular}{|c|c|c|c|c|c|c|c|c|}
\hline \multirow{6}{*}{ 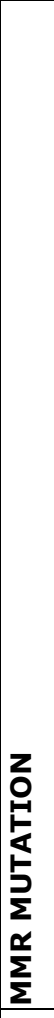 } & $\begin{array}{l}\text { LOE\# \# / } \\
\text { IHC }\end{array}$ & 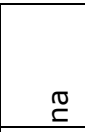 & $\stackrel{\mathbb{2}}{\simeq}$ & $\stackrel{\text { O }}{\complement}$ & $\stackrel{\mathbb{O}}{\mathrm{C}}$ & $\stackrel{\mathbb{O}}{\complement}$ & $\stackrel{\text { Õ }}{\complement}$ & $\stackrel{0}{\simeq}$ \\
\hline & $\begin{array}{l}\text { NGS } \\
\text { confirmed }\end{array}$ & 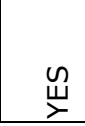 & 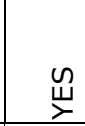 & 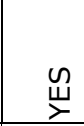 & 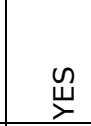 & $\underset{\varpi}{\stackrel{u}{x}}$ & 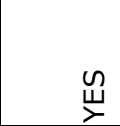 & $\underset{\Perp}{\check{x}}$ \\
\hline & LOVD ID $* * *$ & 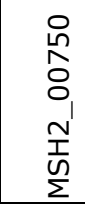 & 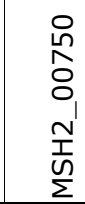 & 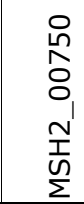 & $\begin{array}{l}N \\
N \\
0 \\
O \\
0 \\
N \\
N \\
\frac{1}{n} \\
\Sigma \\
\Sigma\end{array}$ & $\begin{array}{l}N \\
N \\
O \\
8 \\
0 \\
1 \\
\frac{1}{\mathcal{L}} \\
\frac{N}{\Sigma} \\
\frac{1}{2}\end{array}$ & 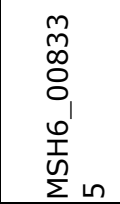 & $\begin{array}{l}m \\
M \\
\infty \\
0 \\
0 \\
0 \\
0 \\
\frac{n}{n} \\
\sum \text { n }\end{array}$ \\
\hline & $\begin{array}{l}\text { protein } \\
\text { change }\end{array}$ & 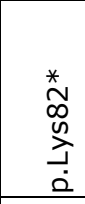 & 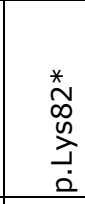 & 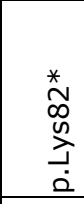 & 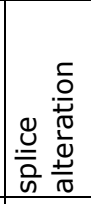 & 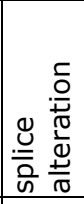 & 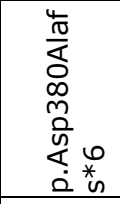 & 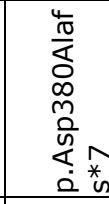 \\
\hline & $\begin{array}{l}\text { DNA } \\
\text { change }\end{array}$ & 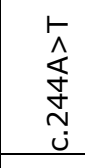 & 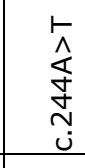 & 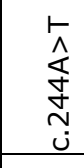 & 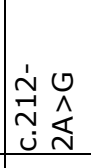 & 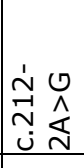 & 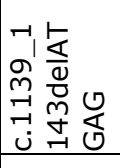 & 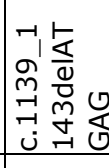 \\
\hline & gene & $\begin{array}{l}\stackrel{N}{T} \\
\stackrel{N}{N}\end{array}$ & $\begin{array}{l}\stackrel{N}{T} \\
\stackrel{N}{\Sigma}\end{array}$ & 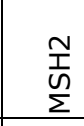 & $\begin{array}{l}\frac{N}{1} \\
\text { N } \\
\Sigma\end{array}$ & $\begin{array}{l}\stackrel{N}{1} \\
\stackrel{n}{\Sigma}\end{array}$ & $\begin{array}{l}\frac{\varphi}{T} \\
\frac{N}{\Sigma}\end{array}$ & $\begin{array}{l}\frac{0}{1} \\
\sum \\
\Sigma\end{array}$ \\
\hline \multirow{9}{*}{ 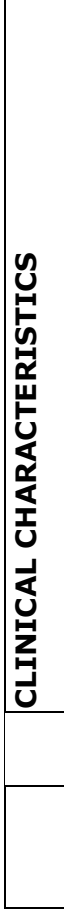 } & $\begin{array}{l}\text { cancer } \\
\text { phenotype }\end{array}$ & 흠 & '̀ & $\begin{array}{l}\grave{o} \\
\text { ㅁ } \\
\end{array}$ & \begin{tabular}{l}
$\grave{0}$ \\
$\circ$ \\
\hdashline
\end{tabular} & $\begin{array}{l}\overline{0} \\
\stackrel{0}{4} \\
\frac{3}{2} \\
\end{array}$ & 흐 & 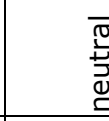 \\
\hline & \begin{tabular}{|l} 
Other \\
tumour \\
sites**
\end{tabular} & & & $\begin{array}{ll}\ddot{a} & m \\
\text { N } & + \\
\dot{u} & u \\
\end{array}$ & & & & \\
\hline & MSI\$ & $\mapsto$ & n & $\stackrel{\text { O }}{\complement}$ & $n$ & $\stackrel{\text { D }}{\complement}$ & $\stackrel{\mathbb{O}}{\mathrm{C}}$ & $\stackrel{\mathbb{O}}{\complement}$ \\
\hline & stage EC & $\underset{-1}{\pi}$ & 우 & $\stackrel{0}{\complement}$ & $\stackrel{\mathbb{Z}}{\complement}$ & $\stackrel{\mathbb{0}}{\simeq}$ & $\stackrel{\pi}{-1}$ & 욱 \\
\hline & grade EC & -1 & $m$ & 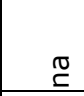 & $\stackrel{\mathbb{O}}{\mathrm{C}}$ & $\stackrel{\mathbb{N}}{\mathrm{C}}$ & -1 & - \\
\hline & type of EC & 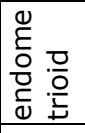 & 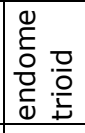 & $\stackrel{0}{\complement}$ & 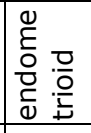 & $\stackrel{\pi}{\check{C}}$ & 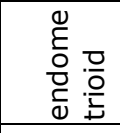 & 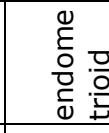 \\
\hline & $\begin{array}{l}\text { Age of EC } \\
\text { diagnosis }\end{array}$ & $\stackrel{\infty}{m}$ & $\stackrel{\varphi}{\forall}$ & $\stackrel{\infty}{m}$ & $\dot{q}$ & ח & g̊ & เก \\
\hline & Rel. & 0 & D & 0 & ш & ш & 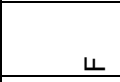 & 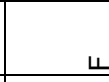 \\
\hline & $\begin{array}{l}\text { ID number } \\
12-04-079\end{array}$ & $\stackrel{-1}{-1}$ & $\underset{\sim}{\sim}$ & $\underset{\sim}{0}$ & I & $\stackrel{\sigma}{-1}$ & $\stackrel{\bullet}{N}$ & $\stackrel{\infty}{\sim}$ \\
\hline
\end{tabular}




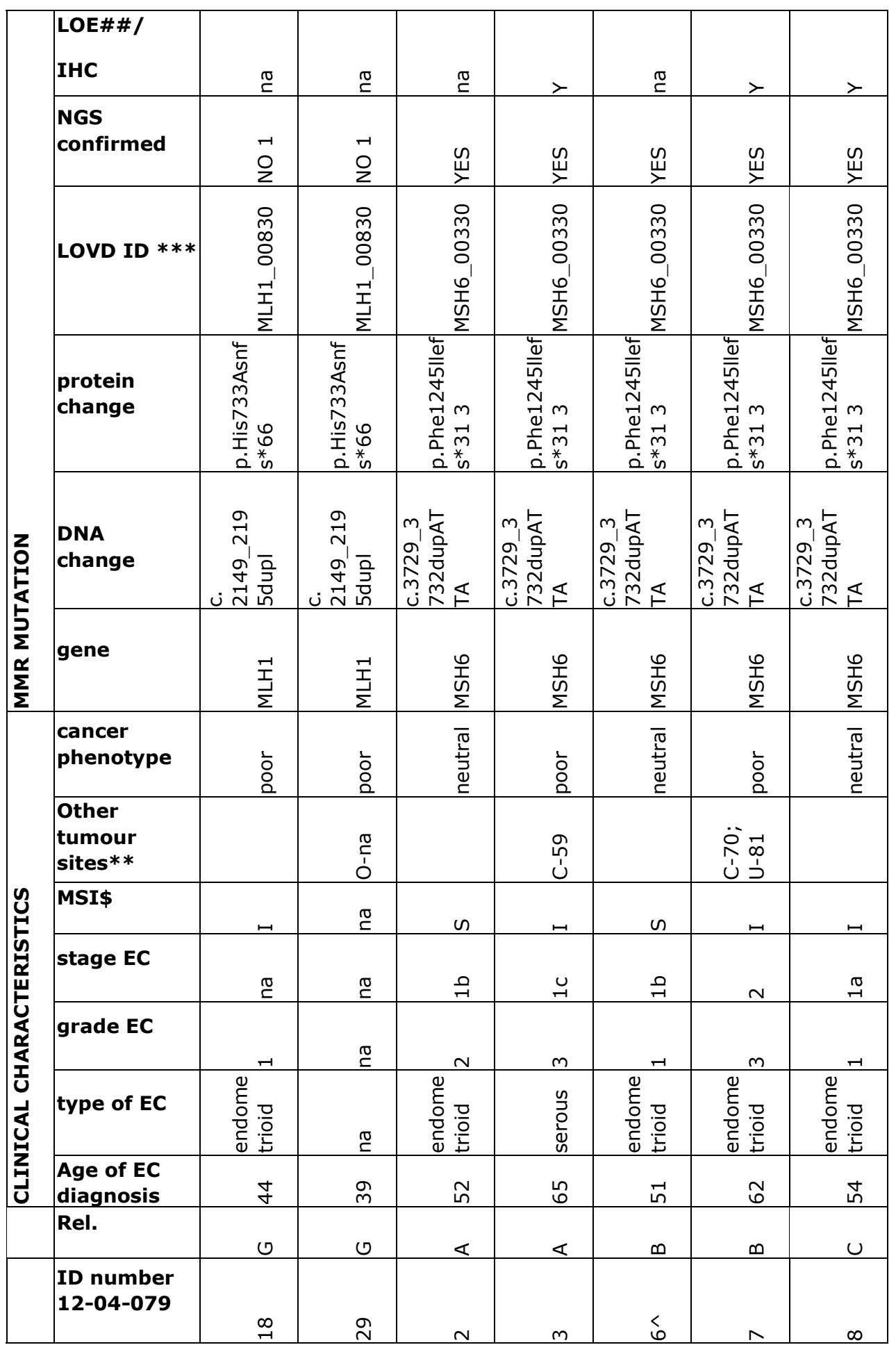




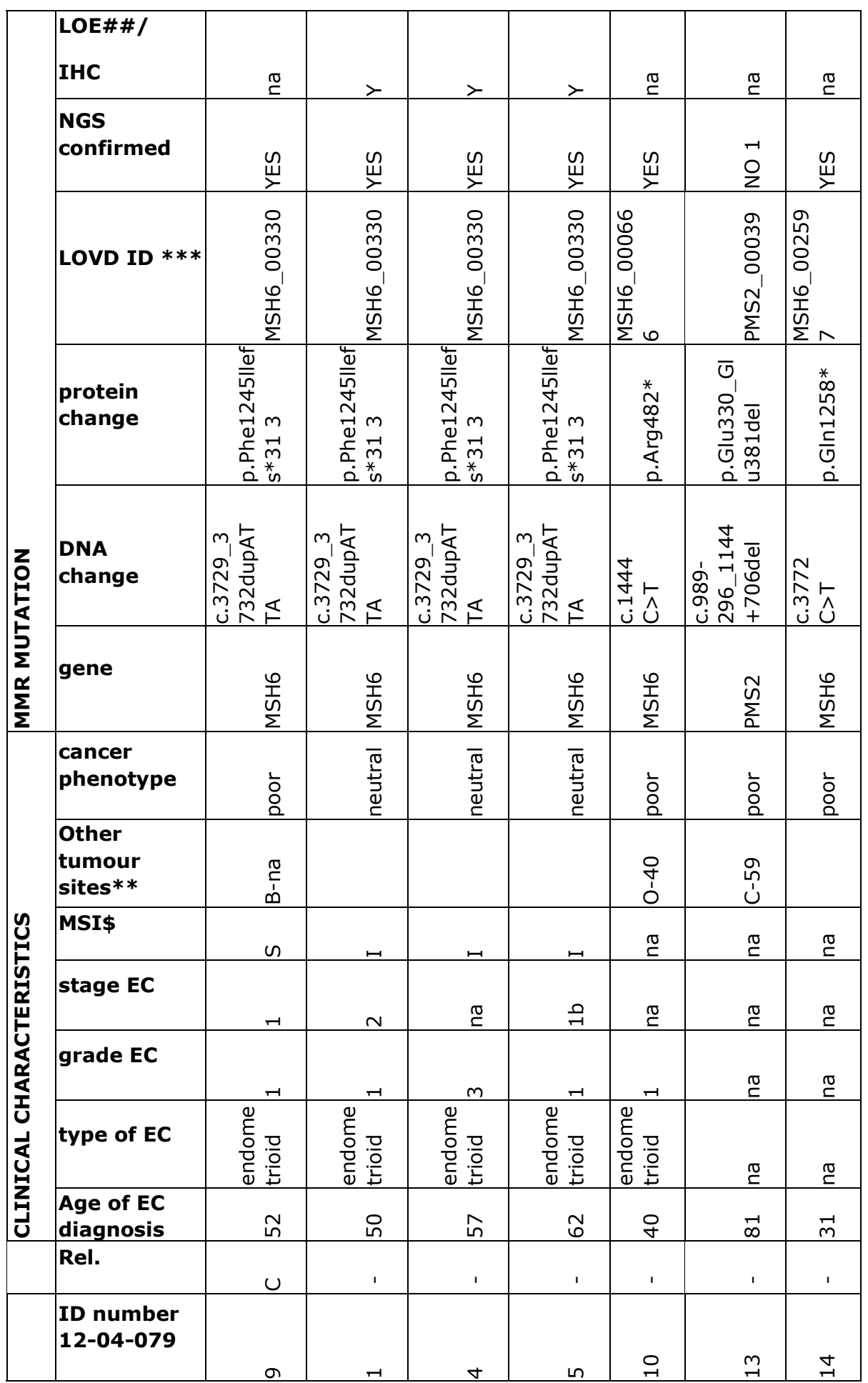




\begin{tabular}{|c|c|c|c|c|c|c|c|c|c|}
\hline \multirow{6}{*}{$\begin{array}{l}\mathbf{Z} \\
\mathbf{0} \\
\mathbf{E} \\
\mathbf{E} \\
\mathbf{L} \\
\mathbf{L} \\
\mathbf{\Sigma} \\
\mathbf{\Sigma} \\
\mathbf{\Sigma} \\
\mathbf{\Sigma}\end{array}$} & $\begin{array}{l}\text { LOE\#\# / } \\
\text { IHC }\end{array}$ & $>$ & $\stackrel{\mathbb{O}}{\complement}$ & $>$ & $\stackrel{\mathbb{R}}{\complement}$ & $>$ & $>$ & $>$ & $\stackrel{\pi}{\complement}$ \\
\hline & $\begin{array}{l}\text { NGS } \\
\text { confirmed }\end{array}$ & $\underset{\varpi}{\sim}$ & 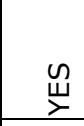 & 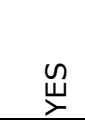 & \# & $\begin{array}{l}N \\
0 \\
z\end{array}$ & $\underset{\Perp}{\check{\nu}}$ & \# & $\stackrel{r}{2}$ \\
\hline & LOVD ID $* * *$ & $\begin{array}{l}0 \\
N \\
\tilde{m} \\
O \\
0 \\
\varphi_{1} \\
\stackrel{1}{1} \\
\underline{n}\end{array}$ & 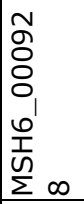 & 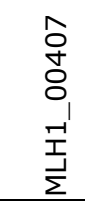 & $\begin{array}{l}10 \\
0 \\
0 \\
0 \\
0 \\
0 \\
0 \\
\frac{1}{\omega} \\
\Sigma\end{array}$ & 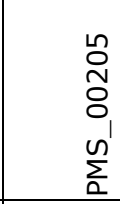 & 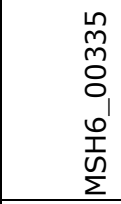 & 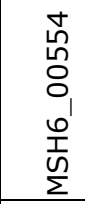 & $\begin{array}{l}0 \\
N \\
8 \\
0 \\
N 1 \\
\frac{1}{\omega} \\
\Sigma\end{array}$ \\
\hline & $\begin{array}{l}\text { protein } \\
\text { change }\end{array}$ & 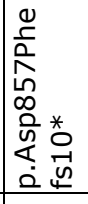 & 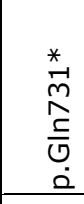 & 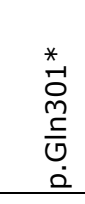 & 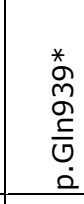 & 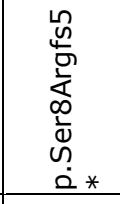 & 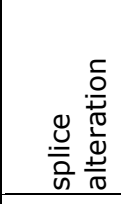 & 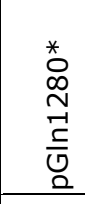 & $\begin{array}{l}\frac{n}{4} \\
\frac{1}{2} \\
\frac{1}{2} \\
N \\
N \\
\frac{0}{4} \\
0 \\
0 \\
0\end{array}$ \\
\hline & $\begin{array}{l}\text { DNA } \\
\text { change }\end{array}$ & 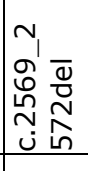 & 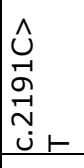 & 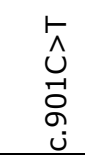 & $\begin{array}{l}n \\
\sim \\
\infty \\
\sim \\
ن \\
ن \\
ن\end{array}$ & 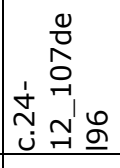 & 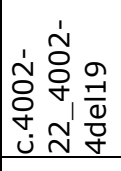 & $\begin{array}{l}\hat{u} \\
\infty \\
m \\
\infty \\
m \\
\sim \\
ن\end{array}$ & 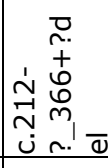 \\
\hline & gene & $\begin{array}{l}\frac{0}{1} \\
\sum \\
\end{array}$ & $\begin{array}{l}\frac{\varphi}{T} \\
\frac{N}{\Sigma}\end{array}$ & $\stackrel{-1}{I}$ & $\begin{array}{l}\frac{0}{1} \\
\frac{N}{n}\end{array}$ & 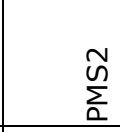 & $\begin{array}{l}\frac{0}{1} \\
\sum \\
\Sigma\end{array}$ & $\begin{array}{l}\frac{0}{1} \\
\sum \\
\Sigma\end{array}$ & $\stackrel{N}{N}$ \\
\hline \multirow{9}{*}{ 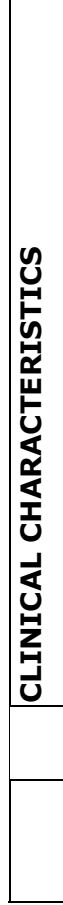 } & $\begin{array}{l}\text { cancer } \\
\text { phenotype }\end{array}$ & 흐 & \begin{tabular}{l}
$\grave{0}$ \\
$\circ$ \\
\hdashline
\end{tabular} & 흐 & 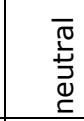 & 흠 & 흐 & '̀ & 흠 \\
\hline & \begin{tabular}{|l} 
Other \\
tumour \\
sites**
\end{tabular} & $\begin{array}{l}\text { g̊ } \\
\stackrel{1}{0} \\
0\end{array}$ & $\begin{array}{l}\text { 우 } \\
\text { ம. } \\
\text { m }\end{array}$ & 足 & & & $\begin{array}{l}0 \\
\text { ப் } \\
\text { ú }\end{array}$ & & \\
\hline & MSI\$ & $n$ & $n$ & $\mapsto$ & $\stackrel{\mathbb{N}}{\complement}$ & $\mapsto$ & $\mapsto$ & $\mapsto$ & n \\
\hline & stage EC & $m$ & $\stackrel{0}{\subseteq}$ & م & $m$ & 우 & 우 & $\stackrel{\mathbb{Z}}{\complement}$ & $\stackrel{0}{\simeq}$ \\
\hline & grade EC & -1 & $\stackrel{\mathbb{O}}{\mathrm{C}}$ & $\rightarrow$ & -1 & -1 & $m$ & -1 & $\stackrel{\overbrace 0}{ᄃ}$ \\
\hline & type of EC & 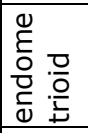 & 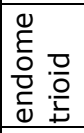 & 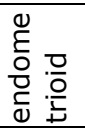 & 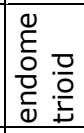 & 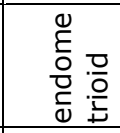 & 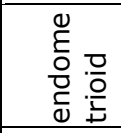 & 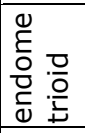 & $\stackrel{\overbrace 0}{\complement}$ \\
\hline & $\begin{array}{l}\text { Age of EC } \\
\text { diagnosis }\end{array}$ & $g$ & ம & $\stackrel{\infty}{\llcorner}$ & 8 & $\stackrel{g}{q}$ & $\overrightarrow{6}$ & \& & 운 \\
\hline & Rel. & 1 & 1 & 1 & 1 & 1 & 1 & I & ' \\
\hline & $\begin{array}{l}\text { ID number } \\
12-04-079\end{array}$ & 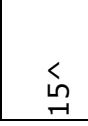 & $\stackrel{\sim}{\sim}$ & $\vec{N}$ & N & $\stackrel{m}{N}$ & $\stackrel{+}{\sim}$ & $\stackrel{\llcorner}{\sim}$ & 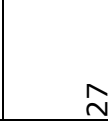 \\
\hline
\end{tabular}




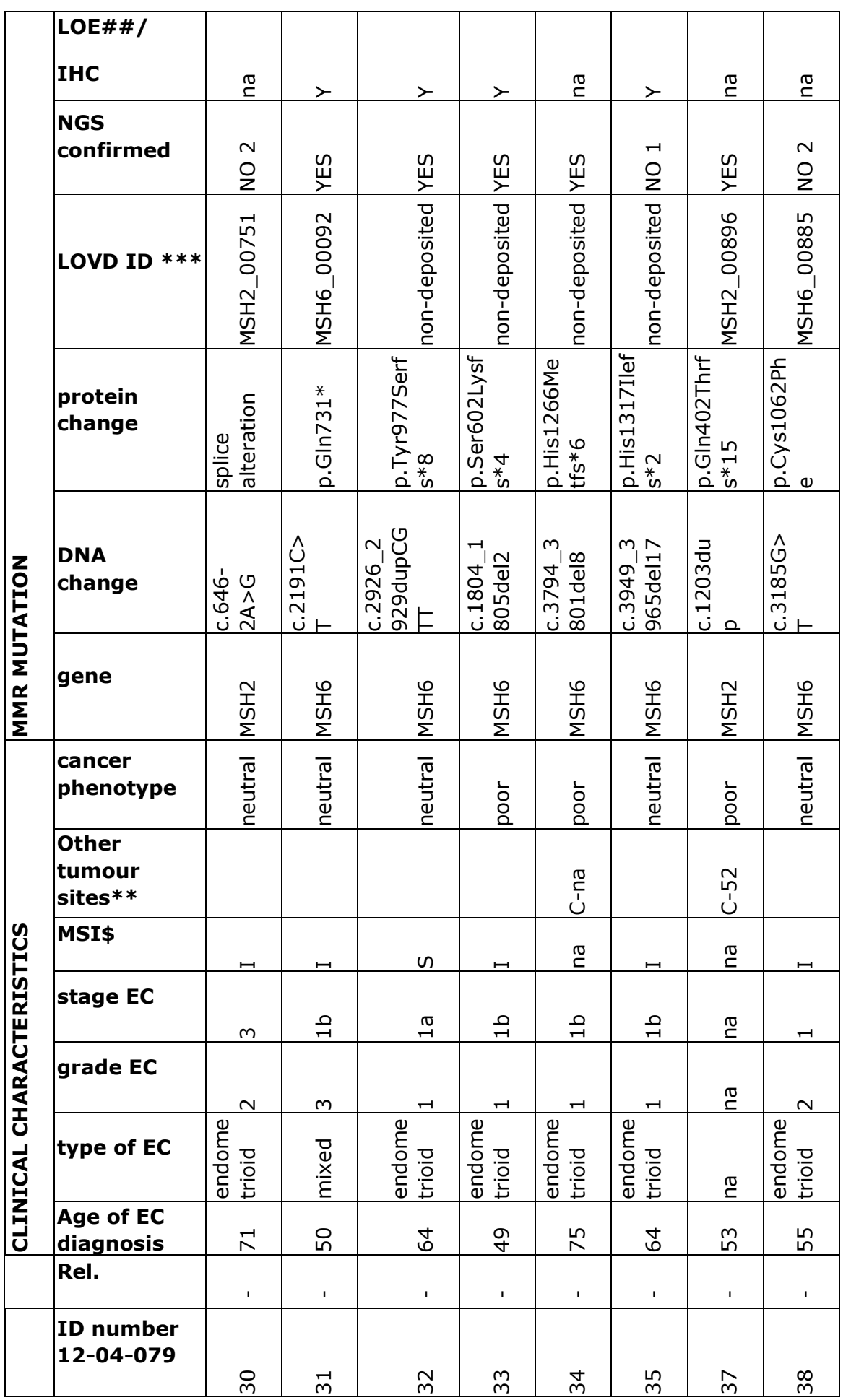




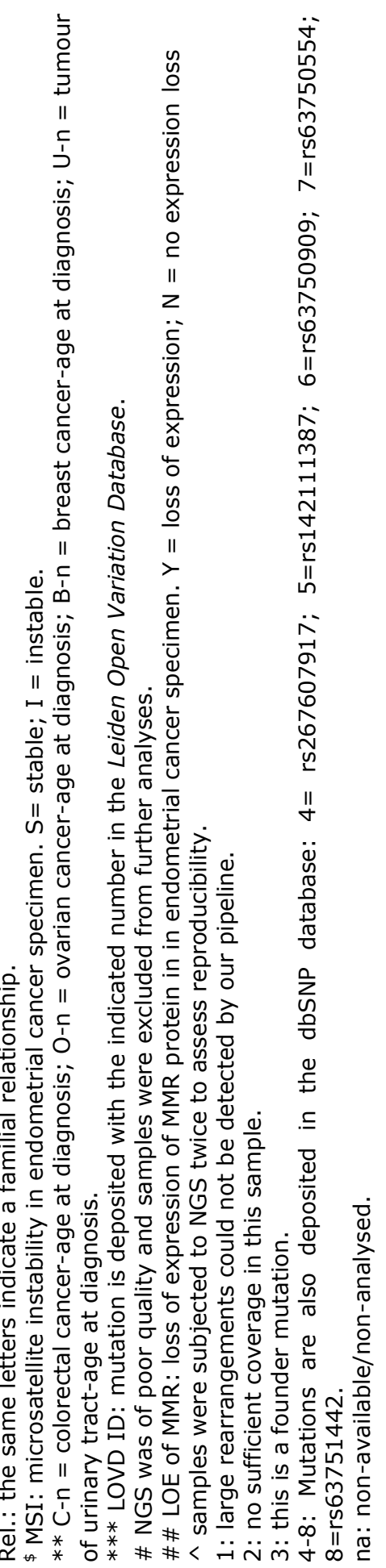




\section{Estrogen and Cancer related 154 Gene-panel}

The coding sequences and splice regions (10 non-coding nucleotides at each exon end) of 154 genes implied in endometrial physiology and carcinogenesis were explored by Next Generation Sequencing (NGS). Table 2 overviews the captured panel and Supplemental Table S1 gives the complete list of genes and the full set of related references.

Table 2: Overview of the panel design of 154 estrogen and cancer associated genes with the variants identified in 35 subjects analysed

\begin{tabular}{|c|c|c|c|c|c|c|c|c|c|}
\hline & \multirow{2}{*}{\multicolumn{2}{|c|}{ Capture plan }} & \multicolumn{7}{|c|}{ Variants identified } \\
\hline & & & \multirow{2}{*}{\begin{tabular}{|c} 
Total \\
No \\
\end{tabular}} & \multicolumn{3}{|c|}{ dbSNP deposited* } & \multicolumn{3}{|c|}{ non-dbSNP deposited } \\
\hline & $\begin{array}{l}\text { No of } \\
\text { genes }\end{array}$ & nt (Kb) & & $\begin{array}{c}\text { Missense } \\
+ \text { non- } \\
\text { sense } \\
\end{array}$ & Silent & \begin{tabular}{|l} 
Intron- \\
exon
\end{tabular} & $\begin{array}{c}\text { Missense + } \\
\text { non-sense } \\
+ \text { ins } / \text { del }\end{array}$ & Silent & $\begin{array}{c}\text { Intron- } \\
\text { exon }\end{array}$ \\
\hline ESTROGEN & 47 & 101.407 & 25 & 9 & 4 & 0 & 11 & 0 & 1 \\
\hline $\begin{array}{c}\text { ligand } \\
\text { metabolism }\end{array}$ & 10 & 13.501 & 3 & 0 & 0 & 0 & 3 & 0 & 0 \\
\hline target genes & 24 & 46.847 & 12 & 6 & 2 & 0 & 3 & 0 & 1 \\
\hline $\begin{array}{c}\text { responsive } \\
\text { genes }\end{array}$ & 7 & 10.539 & 1 & 0 & 0 & 0 & 1 & 0 & 0 \\
\hline co-regulators & 6 & 30.52 & 9 & 3 & 2 & 0 & 4 & 0 & 0 \\
\hline ONCOGENES & 35 & 112.56 & 24 & 3 & 5 & 2 & 8 & 6 & 0 \\
\hline \begin{tabular}{|c|} 
TUMOUR \\
SUPPRESS
\end{tabular} & 63 & 162.306 & 40 & 17 & 9 & 0 & 8 & 4 & 2 \\
\hline OTHER & 9 & 19.395 & 9 & 0 & 3 & 0 & 5 & 0 & 1 \\
\hline TOTAL & 154 & 395.668 & 98 & 29 & 21 & 2 & 32 & 10 & 4 \\
\hline
\end{tabular}

*Variants deposited in the dbSNP database with MAF $<0.001$.

The list of all variants is given in Supplemental Table S3.

Estrogens are important physiological regulators in the endometrium and risk factors for cancer [12]. Hence, the capture panel included 47 genes involved in estrogen (and progesterone) signaling, comprising genes controlling the metabolism and degradation of steroids [12], genes directly targeted by the 
hormone receptors [13-15], genes responsive to steroids $[14,16]$ and genes encoding for co-regulators controlling the hormone receptor transcriptional activity [13-15]. In addition, 35 oncogenes and 63 tumor suppressors were captured [10, 17-19]. NGS and Quality Control analyses are described as Supplemental Materials (Supplemental Methods, Supplemental Figures S1, 2 and Supplemental Table S2). Thirty-seven DNA samples were sequenced but two were excluded due to low quality (12-04-079_22 and 12-04-079_25, Supplemental Materials and Table $\mathbf{1}$ ). As control of NGS and raw data analyses, two samples (12-04-079_6 and 12-04-079_15) were randomly selected and subjected to one additional and independent library preparation and NGS run and they showed $100 \%$ concordance between called variants.

\section{Variant Analyses}

Among the 35 patient's DNA successfully sequenced, 865 independent variants deposited in dbSNP database were identified and each subject carried on average 245 single nucleotide polymorphisms (SNPs; range 207-270). Common SNPs were excluded from further analyses, but since rare dbSNP variants can be relevant in our search for genetic risk modifiers [20, 21], 52 variants with a minor allele frequency (MAF) lower than 0.001 were retrieved (Table 2). Of these, 28 were missense, one non-sense (stop), 21 were silent and two located in noncoding intron/exon boundaries. In addition, 46 identified variants were not reported in dbSNP, and 34 of these were never deposited before in any database (ExAC, Cosmic, LOVD and HGMD were checked; Table 2). Twenty-nine missense, one frame-shift non-sense, one in frame deletion and one in frame insertion of one amino-acid were identified plus 10 silent variants and four changes located in the non-coding regions flanking an exon (Table $\mathbf{2}$ ).

In total, 98 rare variants were identified (dbSNP with MAF <0.001 and non-dbSNP deposited). Considering only variants affecting the amino-acid sequence, there were 57 missense, one amino-acid insertion, one deletion (both in-frame) and two non-sense changes. All variants were heterozygous (Supplemental Table S3). 


\section{Putatively Genetic Risk Modifiers: Risk-Variants}

To predict the functional consequences and potential risk modifying effects, variants were curated using defined criteria [20-22], literature, controlling manually and in combination with bioinformatics tools such as: Alamut (version 2.4, Interactive Biosoftware, including SIFT and PolyPhen-2), Uniprot, PhosphoSitePlus ${ }^{\circledR}$ (for three-dimensional structure and post-translational modifications) [23], NCBI Clinical Variants, ExAC, NHLBI ESP, LOVD, HGMD, Cosmic.

Variants outside the MMR genes were analysed first. The 98 rare variants reported earlier were considered plus three SNPs with a MAF $\geq 0.001$ previously reported as pathogenic or disease related. These were: rs57865060, chromosome position Chr15:75012998:delT, with predicted protein change CYP1B1:p.(Glu229Lys); rs121918303, Chr13:32351535:A > C with predicted protein change RXFP2:p.(Thr222Pro); rs56378716, Chr17:56356502: A > G, with predicted protein change MPO:p.(Met251Thr).

The resulting 101 variants (Supplemental Table S3) were categorized in five groups according to the recommendations of the Dutch and British societies for clinical genetics [21]: categories 1 and $2=$ no effect; $3=$ variants of unknown significance - VUS; 4 = likely pathogenic; $5=$ pathogenic. Silent and non-coding variants were categorized as 1 (none was predicted to affect splicing or other regulatory functions). Conservative amino acid changes were considered unlikely to affect the protein function (category 2), with the exclusion of four variants that had been associated with disease or were predicted to have damaging effects (Alamut prediction tool), which were categorized as 3 . The remaining class 3 variants were all non-conservative protein changes. Eight variants were in category 4, four of which were pathogenic (class 5) for disorders other than endometrial cancer, hence they were considered as class 4 in the present study. Two independent investigators (RA and BMJ, of which BMJ is a Clinical Laboratory Geneticist with expertise in diagnostics and oncogenetics) performed this curation blindly for each other's results. Supplemental Table S3 gives the curation details and the complete variant list. 
Since genetic risk modifiers have modest effects (see discussion), all variants categorized as 3 (provided the presence of a non-conservative amino-acid substitution) or higher were considered and resulted in 54 candidate riskmodifiers. Such variants, referred to as 'risk-variants' from now on, were further characterized for the following features (Supplemental Table S3): $i$ ) the presence of cancer somatic (not silent) mutations in the same protein region (i.e. max $+/-3$ amino acids from the identified risk-variant; Cosmic database). This was considered as an indication that changes in the specific protein region could affect function and predisposition to cancer; ii) the interspecies protein domain conservation; iii) the presence of the same risk-variant among relatives; iv) the occurrence of the risk-variant in relevant protein domains; $v$ ) the predicted consequences based on Alamut, protein in silico analysis, literature mining or the occurrence of other pathogenic variants/SNPs in the same region; vi) we excluded that risk-variants could co-segregate with the familial MMR mutation by verifying their localization on separate chromosomes (Supplemental Table S3).

The majority of the patients carried one (or more) risk-variant in estrogen related genes (19 out of 35 patients, 54\%) and 18 risk-variants were unique (i.e. the same variants occurring in relatives were counted only once: $n=5$ ). Risk-variants in tumor suppressors were observed in 18 patients (49\%), and 25 were unique. One (or more) oncogene contained nucleotide changes in nine patients $(26 \%)$, and nine were unique.

MMR genes also contained three risk-variants beside the familial mutation and these have not been computed in the list of 101 variants given so far (Figure 1A). Two variants, one in PMS2 -rs116788608, Chr7:6035211:T > C, with predicted protein change PMS2:p.(Asp286Gly)- and the second in MSH2 rs41295288, Chr2:47702191:A > G with predicted p.(Asp596Ser)- were on other chromosomes than the familial MMR mutation (MSH6) of these subjects. In contrast, MSH6 variant with predicted protein change p.(Ala25Ser) (rs267608026, Chr2:48010445:G > T) in patient 12-04-079_32 could cosegregate with the MSH6 familial mutation carried by this subject. Besides these three risk-variants, three additional variants in MMR genes with MAF $<0.001$ were classified in category 2 (Figure 1A). 


\section{A. GENE VARIANTS PER PATIENT}

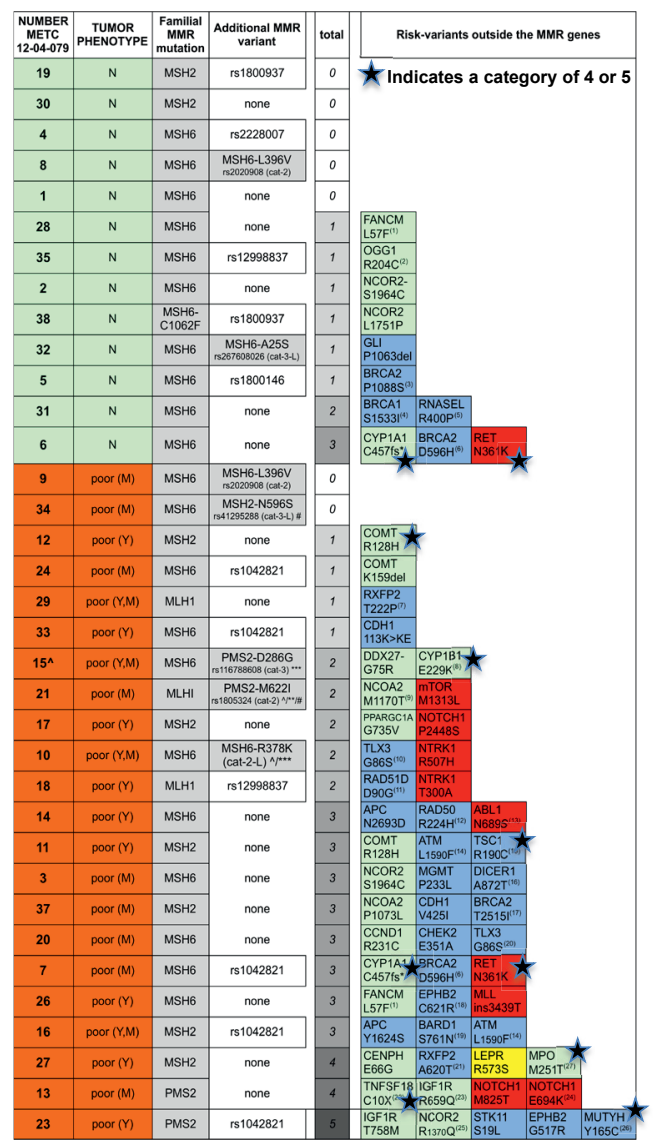

\section{GENE COLOR CODE}

$\square$ Estrogen signalling

Tumour suppressors

Oncogenes

Other

\section{B. KAPLAN-MEIER ANALYSES}

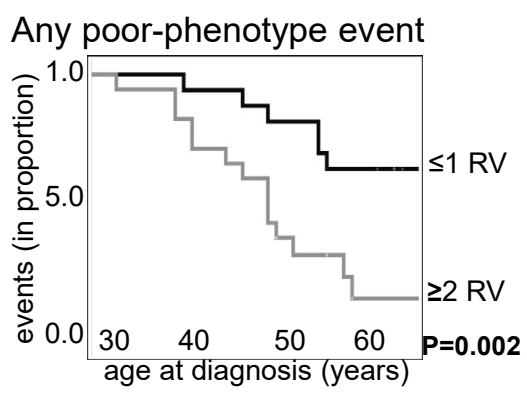

Age endometrial cancer diagnosis

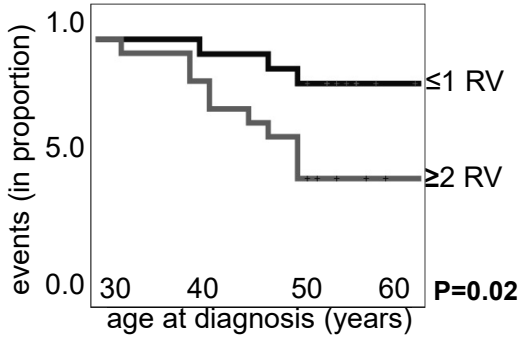

Occurrence of a second tumour

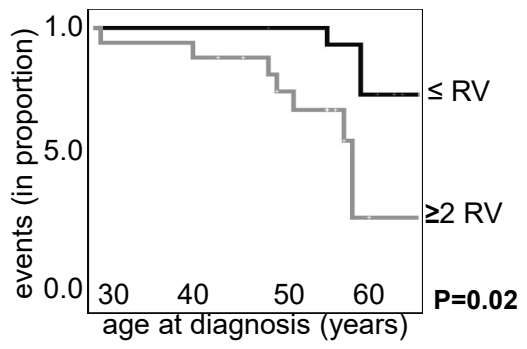

\begin{tabular}{|c|c|c|c|c|c|c|c|c|}
\hline \multirow[t]{2}{*}{$\begin{array}{c}\text { No. } \\
\text { risk- } \\
\text { variants }\end{array}$} & \multirow{2}{*}{$\begin{array}{c}\text { lifespan* } \\
p=n s\end{array}$} & \multirow{2}{*}{$\begin{array}{c}\text { total } \\
n\end{array}$} & \multicolumn{2}{|c|}{$\begin{array}{c}\text { Any poor } \\
\text { event }\end{array}$} & \multicolumn{2}{|c|}{\begin{tabular}{|c|} 
EC before \\
50 years
\end{tabular}} & \multirow{2}{*}{\multicolumn{2}{|c|}{$\begin{array}{l}\text { Second } \\
\text { tumour }\end{array}$}} \\
\hline & & & 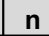 & $\%$ & $n$ & o & & \\
\hline $2(\mathrm{~mol})$ & & $\begin{array}{l}17 \\
18\end{array}$ & 15 & & $\begin{array}{c}3 \\
10\end{array}$ & & & 50.0 \\
\hline
\end{tabular}

Figure 1: The number of class 3 (or more) risk-variants correlates with poor clinical phenotypes. A. Heat map showing the presence of class 3 (or more) risk-variants in each subject with neutral phenotype (green, $\mathrm{N}$ ) or with a poor phenotype (orange, poor; $\mathrm{Y}=$ young age of endometrial cancer diagnosis; $M=$ cancer at multiple sites). The familial MMR mutations (all non-sense but the one carried by subject 12-04-079_38) are indicated (3rd column). 'Additional MRR variants' (4th column) reports the six rare variants (MAF < 0.001 ) found in MMR genes among seven subjects. In particular, MSH6:p.(Arg378Lys) in subject 12-04-079_10 has been already described to be in linkage with the familial MSH6:p.(Arg482*) mutation detected in the patient [24]. 'Total' (5th column) refers to the 
total number of risk-variants outside the MMR genes identified in each patient. The specific risk-variants are reported at the right as gene name and predicted amino-acid change (for full chromosomal coordinates/nucleotide change, see Supplemental Table S3), together with a color code that indicates the gene function. Variants previously reported as (likely) pathogenic (categories 4 and 5) are labeled with a starred ' $P$ '. B. Kaplan-Meier curves showing the occurrence of any poor-clinical phenotype feature (Top chart; first events were only considered for statistic), of the diagnosis of endometrial cancer before 50 (Middle chart), or of the occurrence of a second tumor (Bottom chart; first events were only considered for statistic) in subjects carrying a maximum of one risk-variant (indicated as $\mathrm{RV}$ ) compared to subjects carrying two or more risk-variants outside the MMR gene. The statistical $p$ for significance is computed by Log Rank (Mantel-Cox, Test of equality of survival distributions for the different levels of risk-variants; SPSS).

\section{Risk-variants are Associated with Poor Clinical Phenotypes}

The distribution of MMR pathogenic mutations did not vary between women with poor and neutral clinical phenotypes (Table 3), but patients with poor clinical phenotypes carried more frequently one or more risk-variants outside the MMR genes compared with women with a neutral phenotype (Table $\mathbf{3}$ and Figure 1A).

Table 3: distribution of familial MMR mutations and risk-variants outside the MMR genes (class-3 or more, i.e. putatively risk modifiers) among patients with neutral and poor clinical phenotypes 


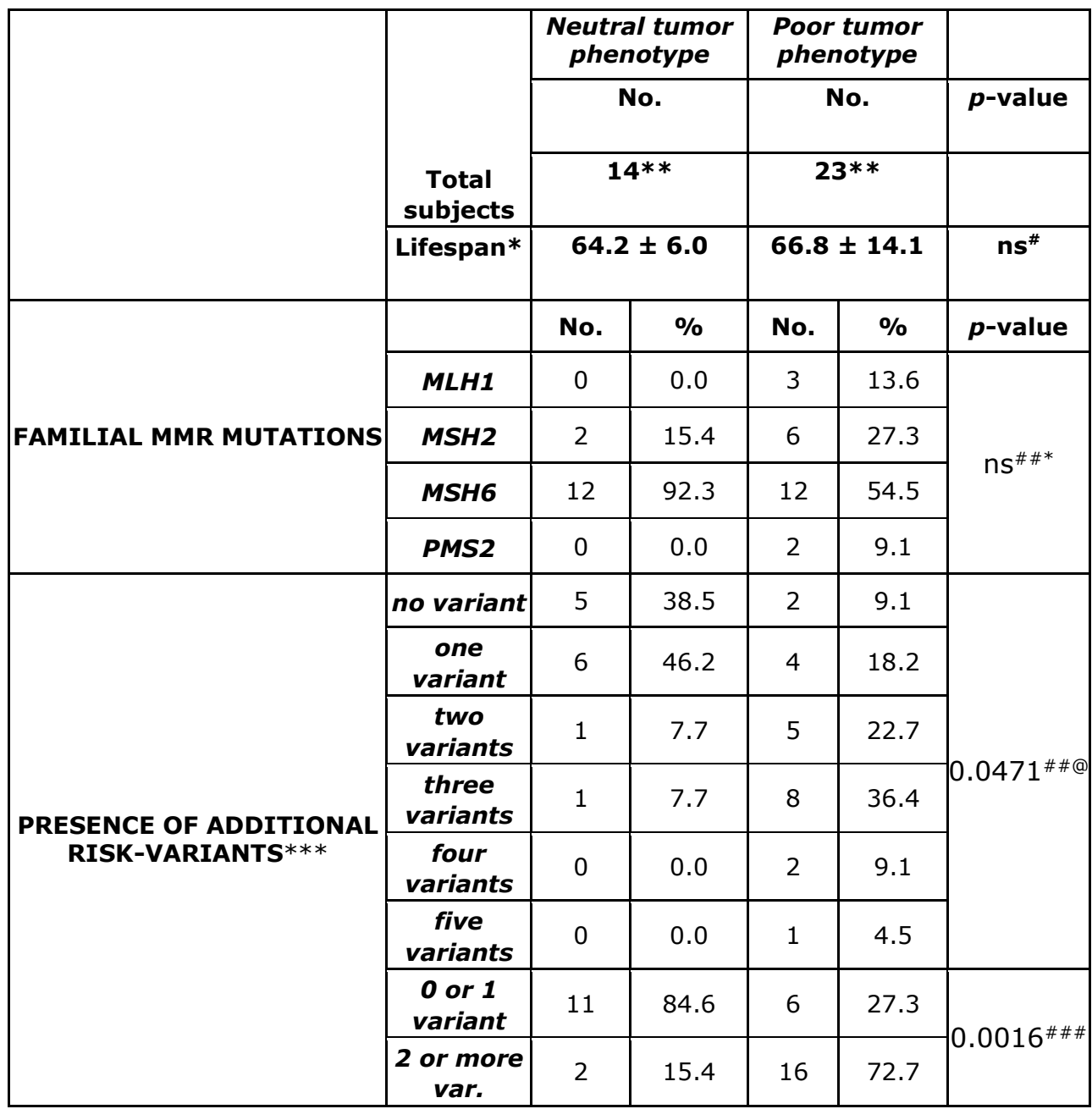

*age of subjects in 2013 was used as lifespan (mean age \pm standard deviation in years).

$* * 13$ and 22 subjects were successfully analysed by NGS.

$* * *$ risk-variants, i.e. class- 3 or higher, in genes other than the MMR were considered.

\#T-test to compare mean values.

\#\#*Chi square, with 3 degrees of freedom.

\#\#@Chi square, with 5 degrees of freedom, Pearson's: 11.225.

\#\#\#Fisher exact test, two sided p-value.

ns $=$ non-significant. 
Cox-regression analysis confirmed that increasing number of risk-variants was associated with a poor clinical phenotype (Supplemental Figure S3). In these Kaplan-Meier analyses the curves of subjects with no or one risk-variant overlap with each other and are separated from the curves of subjects with two, three or more than four risk-variants. Table $\mathbf{3}$ (rows at the bottom) also shows a highest statistical significance by combining these two groups. Because of this net distinction between subjects carrying no or one risk-variant versus women carrying more than two risk-variants, in subsequent Kaplan-Meier cox-regression analyses we combined patients in these two groups (Figure 1B). The presence of two or more risk-variants beside the familial MMR mutation was associated with the occurrence of poor clinical features. As defined earlier, poor clinical features were either a diagnosis of endometrial cancer at a young age $(<50)$, or the onset of multiple cancers. Subsequently, these two characteristics were assessed separately and the presence of two or more risk-variants was associated with both (Figure 1B). The lifespan among patients with neutral and poor clinical phenotypes, extrapolated at 2013 (the date of last update of our database), did not vary significantly between groups (Table 3, Figure 1B and Supplemental Figure S3).

To exclude that multiple risk-variants could co-occur because of co-segregation, we verified that all risk-variants in one subject were located on separate chromosomes. This was confirmed for 16 out of the 18 subjects. Subject 12-04079_14 carried risk-variants Chr5:112179368:A > G (APC:p.(Asn2693Asp)) and Chr5:131915673:G > A (RAD50:p.(Arg224His)) both on chromosome 5 (at a distance of about $90 \mathrm{Mb})$. A second patient (12-04-079_13) carried two riskvariants in the same gene (NOTCH1). Results did not change by considering these four risk-variants as two co-segregating haplotypes.

Three possible risk-variants (category 3 or higher) were identified in MMR genes (grey shaded in Figure 1A), which were not considered in the aforementioned analyses because a linkage with the MMR familial mutation could not be excluded. Nevertheless, when these risk-variants were included, results did not change. In fact, two risk-variants that lied certainly in other chromosomes than the MMR familial mutation - Chr7:6035211:T > C, predicted protein change PMS2:p.(Asp286Gly) and Chr2:47702191:A > G with predicted 
MSH2:p(Asp596Ser)- occurred in patients with a poor clinical phenotype, whereas risk-variant Chr2:48010445:G > T, with predicted change MSH6:p.(Ala25Ser), which could be in linkage with the familial mutation, occurred in subject 12-04079_32 having a neutral clinical phenotype.

Among 15 subjects there were some $1^{\text {st }}$ and $2^{\text {nd }}$ degree parental relationships and they belonged to 7 families. Among the five families in which members had distinct neutral or poor clinical phenotypes, there were three families ( $A, E$ and $F$ ) in which the woman with poor phenotype carried two or more risk-variants and carried more risk-variants than the relative with a neutral phenotype (Supplemental Figure S4). In family $B$, both members carried three risk-variants but the woman with neutral phenotype was significantly younger than the women with poor phenotype, and the occurrence of a poor phenotype later in life cannot be excluded.

\section{Protein Characterization in Tumors}

Immunohistochemistry was used to further characterize the protein expression in a limited number of endometrial tumor specimens carrying the risk-variants (formalin-fixed-paraffin-embedded, FFPE). Risk-variants and relative predicted protein change were: Chr10:43604498:C > A, with predicted protein change RET:p.(Asn361Lys); Chr15:75012998:delT, CYP1A1:p.(Cys457*); Chr15: 99465448:C > T, IGF1R:p.(Thr758Met), Chr12:124821523:G > C, NCOR2:p.(Ser1964Cys); Chr22: 19951274:delAAG， COMT:p.(Lys159del); Chr1:11264 625:T > G, mTOR:p.(Met1313Leu). We will refer from now on to these risk-variants using their predicted protein changes. Results on protein expression in the Lynch tumors were compared with a panel of sporadic endometrial cancers $(n=7)$ and post-menopausal healthy controls $(n=5)$. The presence of the risk-variant-allele in the Lynch tumors was confirmed by Sanger sequence analyses in 12 samples and none showed loss of heterozygosity ( $\mathrm{LOH}$, listed in Supplemental Table S4).

Since we found a substantial number or risk-variants mapping in the estrogen signaling, the expression levels of the estrogen receptor (ER-a) and the Ser-167phosphorylated form, induced by growth factors (including mTOR, IGF1R and RET) 
Chapter 3

and associated with ligand-independent activation [25-27], were assessed. ER-a and phospho-Ser-167-ER-a levels varied between samples, with highest expression in non-cancerous endometrium (both from healthy controls and normal tissues adjacent to cancer cells, i.e. peri-tumoural tissue), intermediate levels in well-differentiated cancers and lowest levels in high-grade lesions (Supplemental Figure S5). The expression of NCOR2, CYP1A1 and COMT, involved in the estrogen signaling (see discussion), and the oncogenes mTOR, RET and IGF1R was high in normal and cancerous endometrium and varied between samples (Figure 2 and Supplemental Figures S5 and S6). 


\section{A. EXPRESSION OF RET}

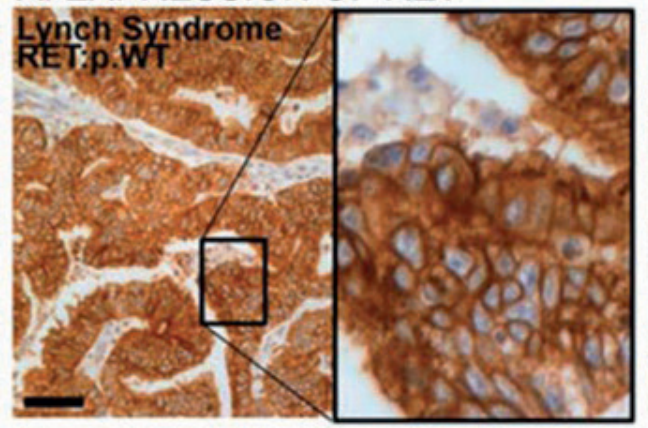

B. EXPRESSION OF CYP1A1

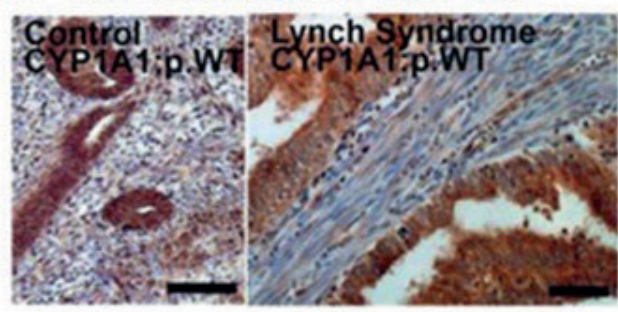

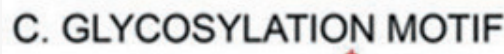

Canonical

IGF1R-N900

IGF1R-N913

IGF1R-N746

IGF1R-N764

IGF1R-N756

IGF1R-T758M

$$
\begin{aligned}
& \mathrm{X}-\mathrm{N}-\mathrm{X}-\mathrm{S} / \mathrm{T} \\
& \mathrm{G}-\mathrm{N}-\mathrm{Y}-\mathrm{T} \\
& \mathrm{G}-\mathrm{N}-\mathrm{G}-\mathrm{S} \\
& \mathrm{A}-\mathrm{N}-\mathrm{T}-\mathrm{T} \\
& \mathrm{Y}-\mathrm{N}-\mathrm{I}-\mathrm{T} \\
& \mathrm{R}-\mathrm{N}-\mathrm{T}-\mathrm{T} \\
& \mathrm{R}-\mathrm{N}-\mathrm{T}-\mathrm{M}
\end{aligned}
$$

\section{E. IGF1R CLSM ANALYSES}

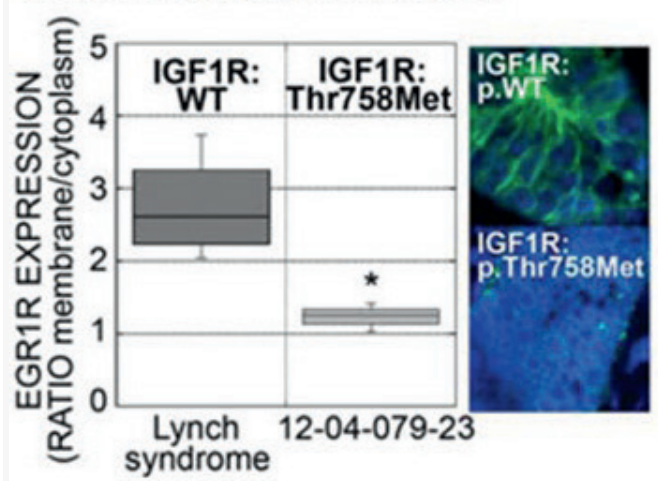

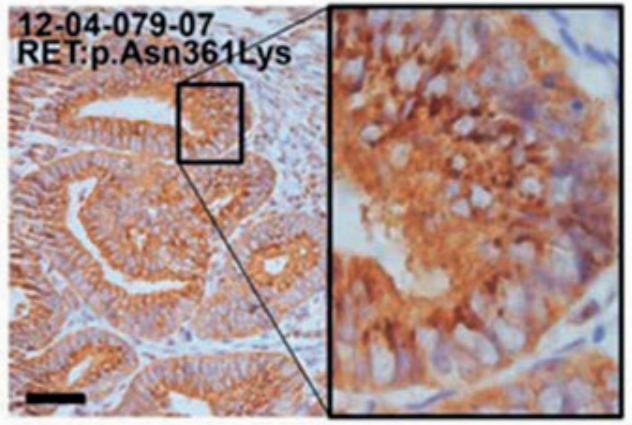

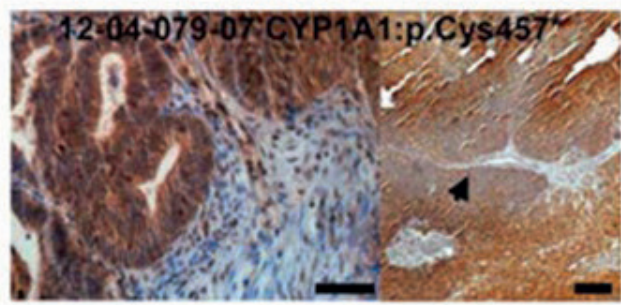

D. IGF1R EXPRESSION
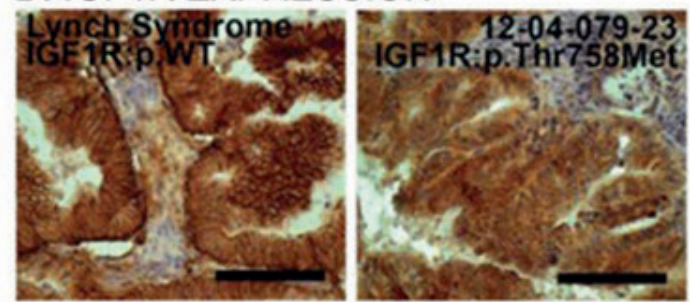

F. pSer-167-ER- $\alpha$ EXPRESSION

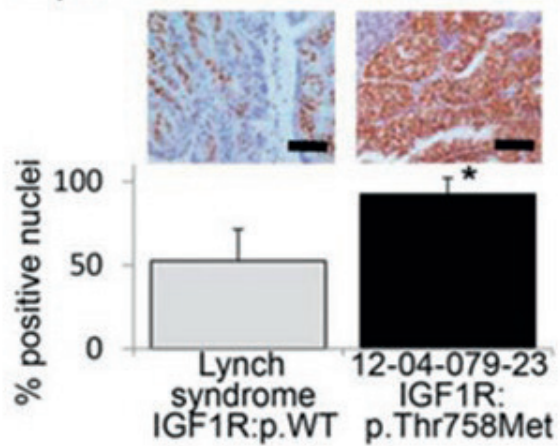

Figure 2: Aberrant expression or localization of RET, CYP1A1 and IGF1R in specimens carrying the respective risk-variants. A. Immunohistochemical images of tumors bearing the common allele (WT) of RET or the risk-variant RET:p.(Asn631Lys). Note the punctuated cytoplasmic pattern in the risk-variant carrying tumor indicative of trapping of the receptor inside the cell. Scale bar $=100 \mu \mathrm{m}$. B. Immunohistochemical images of endometrial 
specimens bearing the common allele (WT) of CYP1A1 (control post-menopausal endometrium and Lynch syndrome endometrial cancer: left images). Right images, CYP1A1 expression on tumor specimen bearing the p.(Cys457*) risk-variant, showing one area with loss of expression (arrowhead). Scale bar $=100 \mu \mathrm{m}$. C. Canonical consensus and the five glycosylation sequences (glycosylation site indicated by red asterisk) in the IGF1R plus the consequent disruption of this consensus caused by risk-variant IGF1R:p.(Thr768Met). D. Immunohistochemical images of tumor specimens with common allele (WT) of IGF1R or with risk-variant p.(Thr758Met). Scale bar $=100$ $\mu \mathrm{m}$. E. Representative confocal laser scanning microscopic (CLSM) images and fluorescence quantification of the membrane/cytoplasmic fractions of IGF1R in tumor with common allele or with p.(Thr758Met) IGF1R. The box plot represents the distribution of four independent samples for common allele and four areas of sample 12-04-079-23 [carrying the p.(Thr758Met) risk-variant]. Asterisk indicates a p-value $<0.05$, Wilcoxon-Mann-Whitney Rank Sum Test. F. Representative immunohistochemical images (top) and quantification of the percentage of nuclei positive to phospho-Ser-167-ER-a among endometrial cancer with wild type IGF1R (the grey bar indicates the mean \pm SD of seven Lynch syndrome tumor specimens) and with IFG1R:p.(Thr758Met) (black bar, mean \pm SD of four independent areas of the tumor from subject 12-04-079-23, bearing the variant). Asterisk indicates a p-value $<0.05$ compared to common allele (t-test). Scale bar $=100 \mu \mathrm{m}$.

The identified risk-variant p.(Asn361Lys) in the RET tyrosine kinase receptor has been previously described in Hirschsprung disease. Asn361 is a N-linked glycosylation site necessary for correct membrane localization of the receptor and mutated RET remains trapped in the endoplasmic reticulum [28]. Immunohistochemistry on sample 12-04-079_7, carrying RET:p.(Asn361Lys), clearly shows an aberrant RET cytoplasmic localization compared with controls where RET is located at the plasma membrane (Figure 2A).

The frame-shift change in CYP1A1 at position 457 is predicted to replace the cysteine heme-binding site [29] with a stop codon thus disrupting the catalytic site and causing premature protein termination. Partial and localized loss of CYP1A1 expression was observed in the tumor carrying this risk-variant (Figure 2B).

Risk-variant p.(Thr758Met) in the tyrosine kinase receptor IGF1R is predicted to abolish the $\mathrm{N}$-linked glycosylation at residue Asn756 (Figure 2C). Glycosylation is needed for correct receptor processing, membrane localization and signal transduction [30]. Specimen 12-04-079_23 carrying this risk-variant showed a decreased membrane immuno-localization of the IGF1R (Figure 2D), which was confirmed with confocal laser scanning microscopy (CLSM; Figure 2E). This observation was concomitant with the highest percentage of phospho-Ser-167- 
ER-a staining (Figure 2F), one of the downstream target of the IGF1R kinase activity, suggestive of a sustained intracellular activation.

\section{Discussion}

Genome-wide approaches [5, 6, 31] have identified modifiers of the cancer risk conferred by pathogenic Lynch syndrome mutations, but additional genetic modifiers are predicted to exist. For instance, genome-wide studies cannot detect rare variants [32] whose risk modifying contribution remains largely undetermined. To identify rare genetic modifiers of the risk of Lynch syndromerelated endometrial cancer, we have screened a panel of 154 genes among a cohort of 35 Lynch syndrome related endometrial cancer patients. Fifty-four variants with putative risk modifying action were selected (risk-variants), which occurred in 40 genes and the presence of two or more risk-variants was associated with poor clinical phenotypes (Figure 1). This result is in line with genome-wide studies that have shown that risk modifying alleles exert larger risks in carriers of multiple variants compared with single-variant carriers [6].

The rationale used to design the 154 gene-panel and the criteria used to select putative risk modifiers derived from recent understandings in cancer biology. Cancer somatic mutation landscapes have shown that while somatic driver mutations inactivate tumor suppressors or activate oncogenes, germ-line predisposing variants have more subtle effects and do not necessarily give a direct growth advantage or cancer phenotype [11]. For these reasons, the designed gene-panel included not only cancer associated-genes but also genes that are broadly involved in the endometrial physiology, like those encoding for proteins controlling the estrogen signaling. In line with this model suggesting a distinction between germ-line predisposing versus somatic driver mutations [11], no (germline) risk-variant was identified among the genes that are most frequently mutated at the somatic level in endometrial cancer, such as PTEN, PIK3CA, CTNNB1, KRAS (all included in the panel). When endometrial cancer somatic mutation landscape (COSMIC database [10]) was aligned to the risk modifiers identified in the present study, MTOR was the first common gene ranking $130^{\text {th }}$ among somatic mutations. STK11 and ATM appeared as risk 
modifiers in our screen and are frequently mutated somatically [9] but not in endometrial cancer (COSMIC database [10]).

Well-established criteria were used to cluster variants in categories $1-5[20,22$, 33]. In addition, we considered that genetic modifiers are expected to have little or no influence in the general population (modest effect-size), while influencing the cancer-risk in predisposed subjects, i.e. in subjects carrying a penetrant cancer mutation [2]. Hence, we considered as putative risk modifiers all variants having a likely-pathogenic or pathogenic effect (category 4/5) but also those with 'unclear significance' (category 3), provided that the were missense leading to non-conservative amino-acid substitution. In this context, the recognition of the role of rare missense changes in cancer susceptibility is growing [34]. Lower categories ( 2 and 1 , unlikely to have any effect at the protein level) were excluded.

Genes such as NOTCH1 (cell-fate determination), NCOR2 and NCOA2 (controlling the activity of ER-a), COMT (detoxification pathway and estrogen degradation), and classical oncogenes (the tyrosine kinase receptors NTRK1 and IGF1R) or tumor suppressors ( $A P C, B R C A 2, E P H B 2$ and $C D H 1$, the latter being already identified as a risk modifier locus in genome-wide studies [6]) carried more than one independent risk-variant, indicating that they are hit frequently. In addition, the pathways where risk-variants were found underscored some signalings in which genetic modifier can be found: the STK11/TSC1/mTOR cascade; the estrogen signaling at ER-a transcriptional control (NCOR2, NCOA2 - both with multiple risk-variants - and PPARGC1A); or at ligand metabolism level, with riskvariants identified in CYP1A1, COMT and CYP1B1, phase I and II detoxifying enzymes degrading also estrogens via the formation of catechol-compounds. The CYP1A1 locus was reported previously as risk modifier for Lynch syndrome colorectal cancer, although data were not confirmed in independent studies [5] and common variants in CYP1A1 and in other genes controlling the estrogen metabolism have been associated with sporadic endometrial cancer [35].

Although it was not possible to assess the segregation of the identified riskvariants with the disease within the families in our cohort (DNA from unaffected relatives with matched life-span was not available), poorer phenotypes tended to 
segregate with a higher number of risk-variants among the small number of patient-relatives included in our cohort (Supplemental Figure S4).

Further immunohistochemical characterization of a limited number of risk-variants showed association with protein changes. The estrogen degrading protein CYP1A1 showed localized loss of expression in the tumor carrying CYP1A1:(p.Cys457*). Tyrosine receptors including RET and IGF1R have important crosstalk's with the estrogen signaling and are relevant to the endometrial physiology [27, 36]. Variant RET:p.(Ans361Lys) impairs RET correct membrane localization [28], and was indeed associated with aberrant cytoplasmic immunoreactivity in this study. Glycosylation of IGF1R is reported to be important for cellular localization, response to anti-IGF therapy and signaling activation [30]. Variant IGF1R:p.Thr758Met, predicted to disrupt one N-linked glycosylation site, was associated with diminished membrane IGF1R localization and increased phosphorylation of ER-a, one of the downstream IGF1R kinase targets. The IGF gene locus (coding for the ligand of IGF1R) has been reported as a genetic risk modifier for Lynch syndrome [5].

In conclusion, we propose a gene-panel that contains potential genetic risk modifiers of Lynch syndrome related endometrial cancer in our population. Riskvariants that are likely pathogenic have been identified and assessing their segregation with the disease phenotypes can be relevant to the family members and the subjects assisted at our center.

For implications outside the families of the present investigation, the development of gene-panels in diagnostics is technically desirable compared to complete genome sequencing because it allows achieving sufficient coverage, cost effectiveness and simplicity in analyses [11, 20]. Therefore, it is intriguing to validate these data in independent populations and examine how this panel will translate to other ethnicities. The fact that a number of risk modifiers identified here correspond to loci that were previously characterized in genome-wide investigations is encouraging. Additionally, it is possible to improve our panel design by including genes recently identified as risk modifiers in genome-wide studies [6, 37]. 


\section{Material and Methods}

\section{Ethical Statement}

Investigation has been conducted in accordance with the ethical standards, according to the Declaration of Helsinki and according to national and international guidelines. Protocols have been approved by the authors' institutional medical ethical committee (see below for details).

\section{Patient Population}

Thirty-seven Dutch Caucasian women from 29 families who developed hereditary endometrial cancer and carried a germ-line mismatch repair (MMR) gene mutation were enrolled (Table 1). Women were counseled at the Maastricht or Leiden Medical Centers (2011-2013) because of a Lynch syndrome family history, a Lynch syndrome mutation or a Lynch Syndrome diagnosis (Bethesda criteria). Genomic DNA was used for Next Generation Sequencing (NGS) analyses. All women had given consent to use their DNA for research. The local medical ethical committee approved the protocol (METC 12-04-079).

Archival formalin-fixed-paraffin-embedded (FFPE) tissues of 12 women's tumor specimen were retrieved from the hospitals that performed the hysterectomy and they were used for DNA analyses, loss of heterozygosity ( $\mathrm{LOH} ; n=12)$ and histology/immunohistochemistry $(n=8)$. Seven sporadic endometrial cancers and five postmenopausal healthy controls were randomly selected from our tissuebank [12] and used as control for immunohistochemistry (local medical ethical committee tissue-bank protocol approval: METC-14-04-003).

\section{Gene-panel and NGS}

Coding sequences plus intron/exon boundaries of 2012 regions from 154 genes (396kB; Table 2, Supplemental Table S1) and 57 off-target control regions (84kB) were designed and captured with Haloplex platform (Agilent Technologies, Ratingen, Germany). Illumina HiSeq NGS (Illumina, San Diego, USA) was used for sequencing and reads were aligned against the reference genome (GRCh37/hg19). Variants were called using NextGene (Softgenetics, State College, USA), SureCall software packages (Agilent Technologies, Ratingen, 
Germany) plus manual checking, which resulted in $0 \%$ false positives (confirmed by Sanger analyses) and in the successful detection of all re- sequenced pathogenic MMR mutations suitable to be detected (Supplemental Methods). Sanger sequencing was performed using BigDye Termination v.1.1. (Life Technologies, Bleiswijk, Netherlands). Detailed descriptions of the gene-panel design, capture, library preparation, NGS, variant calling and quality controls are given in Supplemental Methods. The dbSNP142 - The National Center for Biotechnology Information (NCBI) was used to identify deposited polymorphisms and variants.

\section{Immunohistochemistry, Confocal Laser Scanning Microscopy (CLSM) and LOH}

Standard protocols $[12,38]$ and antibody manufacturer's instructions were used for immunohistochemistry. Antigens were retrieved with tris-EDTA buffer. Antibodies used: estrogen receptor-a (ERa; monoclonal D-5, 1:100; Dako, Glostrup, Denmark), IGF1R (1:400; monoclonal; Novus Biologicals, Littleton, USA), CYP1A1 (1:1000; polyclonal; Sigma-Aldrich, St. Louis, USA), COMT (1:100; polyclonal; Sigma-Aldrich, St. Louis, USA), Phospho-ERa $\operatorname{ser} 167$ (1:50; monoclonal; Cell Signaling Technology, Danvers, USA), RET (1:500; polyclonal; Sigma-Aldrich, St. Louis, USA). Chemate Envision and 3,3-diaminobenzidine (Dako, Glostrup, Denmark) were used to visualize antibody binding. Immunoscores were assessed by two independent investigators (DB and RA) as described [12]. Protocols for SMRT, Phospho-S6 Ribosomal protein-ser235/236 and mTOR staining are given in Supplemental Figure S6.

Confocal laser scanning microscopy (CLSM) was performed on $4 \mu \mathrm{m}$ thick FFPE tissue sections. Anti IGF1R (1:250; monoclonal; Novus Biologicals, Littleton, U.S.A.) followed by rabbit-a-mouse IgG-FITC (1:100, Dako, Glostrup, Denmark) were used. CLSM was performed with Leica CTR 4000/CTC SPE and the Application Suite/advance fluorescence software (Leica Microsystems B.V., Rijswijk, Netherlands). Image-J program ( $v$ 1.48, National Institutes of Health, USA) was used for image analyses. For LOH, an expert pathologist (VdVKK) identified the tumor regions from histological sections of FFPE materials, which were resected 
for genomic DNA isolation (QIAamp DNA FFPE tissue kit, Leusden, Netherlands) and Sanger sequencing. At least two separate tumor regions were analysed.

\section{Statistical Analyses}

Kaleidograph ( $v$ 4.1, Synergy Software, Reading, USA), SPSS ( $v$ 22, IBM Corporation, Armonk, USA) and the online Simple Interactive Statistical Analysis (SISA) were used as indicated in the text.

\section{URLS}

\section{Genebrowsers}

Ensembl Genome Browser: http://www.ensembl.org;

NCBI: http://ncbi.nlm.nih.gov; University of California Santa Cruz: UCSC Genome Browser: http://genome.ucsc.edu.

\section{Variant Analyses}

dbSNP database Human Build 142: http://ncbi.nlm.nih.gov; Exome Aggregation Consortium: ExAC, v 0.3, (Cambridge, MA, USA; February 2015): http://exac.broadinstitute.org/; Exome Variant Server, NHLBI GO Exome Sequencing Project (ESP, release ESP6500SI-V2, March-2015), Seattle, USA: http://evs.gs.washington.edu/EVS; Leiden Open Variation Database: LOVD 3.0: lovd.nl; The Human Gene Mutation Database: HGMD (Cardiff University): http://www.hgmd.cf.ac.uk; Genome of Netherlands project, release 5: http://www.nlgenome.nl [39]; Catalogue Of Somatic Mutations In Cancer:

Cosmic (Sanger Institute): http://cancer.sanger.ac.uk;

PhosphoSitePlus ${ }^{\circledR}$ : http://www.phosphosite.org [23].

\section{Gene Ontology, Expression Information}

NCBI: http://ncbi.nIm.nih.gov; UniProt

Knowledgebase: http://www.uniprot.org/uniprot; GeneCards (v 3.12.354 March 2015, Weizmann Institute of Science): http://www.genecards.org.

\section{Statistics}

SISA: http://www.quantitativeskills.com/sisa/. 


\section{Acknowledgement and Funding}

The authors would like to thank the personnel from the pathology departments of the hospitals that kindly provided FFPE archival tissues from the patients: dr. S. Wouda (Viecuri Hospital, Venlo), dr. C. Huysentruyt (PAMM, Veldhoven), and the colleagues from the hospitals of Helmond (Elkerliek Ziekenhuis) and Nijmegen (Canisius-Wilhelmina Ziekenhuis). Special thanks go to G. Roemen, dr B. de Vries and L. Kooreman, dept. Pathology (MUMC), for helping with the archival materials and FFPE DNA isolation in our center; dr T. van Gorp and K. Cornel, dept. of Gynaecology (MUMC), for helping with the statistic analyses; dr. J. Broers and M. Kamps, dept. Molecular and Cell Biology (MUMC), for assistance with the CLSM. The authors would like to thank the NHLBI GO Exome Sequencing Project and its on-going studies, which produced and provided exome variant calls for comparison. The on-going studies are: the Lung GO Sequencing Project (HL102923), the WHI Sequencing Project (HL-102924), the Broad GO Sequencing Project (HL-102925), the Seattle GO Sequencing Project (HL-102926) and the Heart GO Sequencing Project (HL-103010). The authors would like to thank also the Exome Aggregation Consortium and the groups that provided exome variant data for comparison (a full list of contributing groups can be found at http://exac.broadinstitute.org/about). Finally, we are grateful to all women who agreed to have their data and biological specimens used for research. This research received no specific grant from any funding agency in the public, commercial or not-for-profit sectors. The study was sponsored by internal funds of the institute GROW - School for Oncology and Developmental Biology granted to $B M J, K R F, G G E B$, RA.

\section{Conflicts of Interest}

None of the authors has any conflict of interest to declare. 


\section{References}

1. Weissman, S.M., et al., Identification of individuals at risk for Lynch syndrome using targeted evaluations and genetic testing: National Society of Genetic Counselors and the Collaborative Group of the Americas on Inherited Colorectal Cancer joint practice guideline. J Genet Couns, 2012. 21(4): p. 484-93.

2. Antoniou, A.C. and G. Chenevix-Trench, Common genetic variants and cancer risk in Mendelian cancer syndromes. Curr Opin Genet Dev, 2010. 20(3): p. 299-307.

3. Gaudet, M.M., et al., Identification of a BRCA2-specific modifier locus at $6 p 24$ related to breast cancer risk. PLoS Genet, 2013. 9(3): p. e1003173.

4. Easton, D.F., et al., Gene-panel sequencing and the prediction of breastcancer risk. N Engl J Med, 2015. 372(23): p. 2243-57.

5. Houlle, S., et al., Evaluation of Lynch syndrome modifier genes in 748 MMR mutation carriers. Eur J Hum Genet, 2011. 19(8): p. 887-92.

6. Study, C., et al., Meta-analysis of genome-wide association data identifies four new susceptibility loci for colorectal cancer. Nat Genet, 2008. 40(12): p. $1426-35$.

7. Tomlinson, I., et al., A genome-wide association scan of tag SNPS identifies a susceptibility variant for colorectal cancer at 8q24.21. Nat Genet, 2007. 39(8): p. 984-8.

8. Tomlinson, I.P., et al., $A$ genome-wide association study identifies colorectal cancer susceptibility loci on chromosomes 10p14 and 8q23.3. Nat Genet, 2008. 40(5): p. 623-30.

9. Greenman, C., et al., Patterns of somatic mutation in human cancer genomes. Nature, 2007. 446(7132): p. 153-8.

10. Lawrence, M.S., et al., Discovery and saturation analysis of cancer genes across 21 tumour types. Nature, 2014. 505(7484): p. 495-501.

11. Vogelstein, B., et al., Cancer genome landscapes. Science, 2013. 339(6127): p. 1546-58.

12. Cornel, K.M., et al., Overexpression of 17beta-hydroxysteroid dehydrogenase type 1 increases the exposure of endometrial cancer to 17beta-estradiol. J Clin Endocrinol Metab, 2012. 97(4): p. E591-601.

13. Johnson, A.B. and B.W. O'Malley, ERasing breast cancer resistance through the kinome. Nat Med, 2011. 17(6): p. 660-1.

14. Romano, A., et al., Identification of novel ER-alpha target genes in breast cancer cells: gene- and cell-selective co-regulator recruitment at target promoters determines the response to 17 beta-estradiol and tamoxifen. Mol Cell Endocrinol, 2010. 314(1): p. 90-100.

15. Zwart, W., V. Theodorou, and J.S. Carroll, Estrogen receptor-positive breast cancer: a multidisciplinary challenge. Wiley Interdiscip Rev Syst Biol Med, 2011. 3(2): p. 216-30. 
16. Groothuis, P.G., et al., Estrogen and the endometrium: lessons learned from gene expression profiling in rodents and human. Hum Reprod Update, 2007. 13(4): p. 405-17.

17. Pritchard, C.C., et al., ColoSeq provides comprehensive lynch and polyposis syndrome mutational analysis using massively parallel sequencing. J Mol Diagn, 2012. 14(4): p. 357-66.

18. Tamborero, D., et al., Comprehensive identification of mutational cancer driver genes across 12 tumor types. Sci Rep, 2013. 3: p. 2650.

19. Salvesen, H.B., et al., Integrated genomic profiling of endometrial carcinoma associates aggressive tumors with indicators of PI3 kinase activation. Proc Natl Acad Sci U S A, 2009. 106(12): p. 4834-9.

20. Van Allen, E.M., N. Wagle, and M.A. Levy, Clinical analysis and interpretation of cancer genome data. J Clin Oncol, 2013. 31(15): p. 1825-33.

21. Rattenberry, E., et al., A comprehensive next generation sequencingbased genetic testing strategy to improve diagnosis of inherited pheochromocytoma and paraganglioma. J Clin Endocrinol Metab, 2013. 98(7): p. E1248-56.

22. Cheon, J.Y., J. Mozersky, and R. Cook-Deegan, Variants of uncertain significance in BRCA: a harbinger of ethical and policy issues to come? Genome Med, 2014. 6(12): p. 121.

23. Hornbeck, P.V., et al., PhosphoSitePlus: a comprehensive resource for investigating the structure and function of experimentally determined post-translational modifications in man and mouse. Nucleic Acids Res, 2012. 40(Database issue): p. D261-70.

24. Hendriks, Y.M., et al., Cancer risk in hereditary nonpolyposis colorectal cancer due to MSH6 mutations: impact on counseling and surveillance. Gastroenterology, 2004. 127(1): p. 17-25.

25. Borrello, M.G., et al., RET inhibition: implications in cancer therapy. Expert Opin Ther Targets, 2013. 17(4): p. 403-19.

26. Yamnik, R.L., et al., S6 kinase 1 regulates estrogen receptor alpha in control of breast cancer cell proliferation. J Biol Chem, 2009. 284(10): p. 6361-9.

27. Martin, M.B., et al., A role for Akt in mediating the estrogenic functions of epidermal growth factor and insulin-like growth factor I. Endocrinology, 2000. 141(12): p. 4503-11.

28. Kjaer, S., et al., Mammal-restricted elements predispose human RET to folding impairment by HSCR mutations. Nat Struct Mol Biol, 2010. 17(6): p. 726-31.

29. Walsh, A.A., G.D. Szklarz, and E.E. Scott, Human cytochrome P450 1A1 structure and utility in understanding drug and xenobiotic metabolism. J Biol Chem, 2013. 288(18): p. 12932-43.

30. Kim, J.G., et al., Heterodimerization of glycosylated insulin-like growth factor-1 receptors and insulin receptors in cancer cells sensitive to antiIGF1R antibody. PLoS One, 2012. 7(3): p. e33322. 
31. Bellido, F., et al., Genetic variant in the telomerase gene modifies cancer risk in Lynch syndrome. Eur J Hum Genet, 2013. 21(5): p. 511-6.

32. Bamshad, M.J., et al., Exome sequencing as a tool for Mendelian disease gene discovery. Nat Rev Genet, 2011. 12(11): p. 745-55.

33. Dewey, F.E., et al., Clinical interpretation and implications of wholegenome sequencing. JAMA, 2014. 311(10): p. 1035-45.

34. Tavtigian, S.V. and G. Chenevix-Trench, Growing recognition of the role for rare missense substitutions in breast cancer susceptibility. Biomark Med, 2014. 8(4): p. 589-603.

35. Yang, H.P., et al., Common genetic variation in the sex hormone metabolic pathway and endometrial cancer risk: pathway-based evaluation of candidate genes. Carcinogenesis, 2010. 31(5): p. 827-33.

36. Boulay, A., et al., The Ret receptor tyrosine kinase pathway functionally interacts with the ERalpha pathway in breast cancer. Cancer Res, 2008. 68(10): p. 3743-51.

37. Wijnen, J.T., et al., Chromosome 8q23.3 and 11q23.1 variants modify colorectal cancer risk in Lynch syndrome. Gastroenterology, 2009. 136(1): p. 131-7.

38. Dassen, $\mathrm{H}$. , et al., Olfactomedin-4 regulation by estrogen in the human endometrium requires epidermal growth factor signaling. Am J Pathol, 2010. 177(5): p. 2495-508.

39. Genome of the Netherlands, C., Whole-genome sequence variation, population structure and demographic history of the Dutch population. Nat Genet, 2014. 46(8): p. 818-25. 


\section{Supplementary Data}

\section{Customized gene-panel}

Customized gene-panel A panel of 154 endometrial physiology and cancer related genes was compiled based on literature and own experience [10, 12-19]. Genes involved in estrogen and progesterone signaling, involved in the metabolism of steroid hormones plus oncogenes and tumor suppressors were included. For the complete gene-panel description and full set of references see Supplemental Table S1. The coding sequences plus intron/exon boundaries based on GenBank and CCDS records were captured (The National Center for Biotechnology). Captured sequences were designed with Haloplex Design Wizard Tool (Agilent Technologies, Ratingen, Germany) using Illumina100 as library type. The designed panel consisted of 396kB from 2012 target regions. As control for off target DNA, 57 additional regions mapping in introns or inter-genic DNA sequences were captured as well $(84 \mathrm{kB})$. Parameters were optimized to the latest targeted exome capture platform Haloplex (Agilent Technologies, Ratingen, Germany) and Illumina HiSeq NGS (Illumina, San Diego, CA, USA).

\section{Next-generation sequencing - NGS}

Genomic DNA was quantified, assessed for quality by Qubit measurement (Life Technologies, Bleiswijk, Netherlands) and for degradation on $1.5 \%$ agarose gel. As positive control for the sequencing reactions, the gDNAcontrol from Agilent was used, and consisted of material from a single Caucasian subject isolated from an early passage lymphoblastic cell line. Target DNA sequences were captured with Haloplex Target Enrichment System (Agilent Technologies, Ratingen, Germany) and barcoded libraries were made according to the manufacturer's protocol. Library quality and DNA content were evaluated on a 2100 Bioanalyzer using the High Sensitivity DNA Kit (Agilent Technologies, Ratingen, Germany). Library clusters were generated using the TruSeq ${ }^{\circledR}$ PE Cluster Kit V3 according to the manufacturer's protocol (Illumina, San Diego, CA, USA) and the paired-end library was sequenced in $2 \times 100$ cycles paired-end sequencing by synthesis (SBS) using

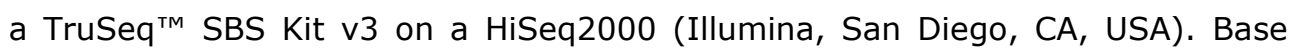
calling was done by using Casava 1.8 (Illumina, San Diego, CA, USA) and sequence reads were aligned against the human reference genome assembly 
GRCh37/hg19 (UCSC Genome Browser on Human Feb. 2009). Total target region size was 480052 base pairs (bp) and after sequencing a total of covered target regions of $98.4 \%$ could be analyzed (472542 bp).

\section{Quality assessment}

Quality assessment of sequencing reactions Quality Control (QC) analysis on coverage was performed by Surecall (Supplemental Figure S1). A threshold for read depth was set of at least 50 times, and for coverage at $90 \%$ of the original regions of interest. Two samples did not match these criteria and were excluded (12-04-079_22 and 12-04-079_25, Supplemental Figure S1 and Table I). Sample 12-04-079_31 was of borderline sufficient quality with a depth of 50 times for about $80 \%$ of the regions of interest. This sample was retained because the familial MMR mutation could be retrieved and Sanger sequencing confirmed the validity of the additional variants found. The remaining 34 samples were covered more than 50 times in every nucleotide position, over $95 \%$ of the region of interests were covered and could be analyzed. Each region was covered on average 2309 times. Supplemental Figure S2 gives the average coverage per region in our samples, and Supplemental Table S2 reports the coverage information per patient per region of interest.

\section{Variant calling and validation}

NextGene software package (Softgenetics, State College, PA, USA) on unique reads with basic settings and SureCall (Agilent Technologies, Ratingen, Germany) were used for raw data analyses. Alignment and variant calling were performed on unique reads with default settings, using a filter set on the region of interest file provided by the Agilent-Haloplex report. To adjust the settings for variant calling, over 70 variants were analyzed by Sanger sequencing (not shown). After this preliminary optimization of variant calling, we further optimized the variant calling pipeline by integrating results from Nextgene and SureCall software's. This strategy resulted in a highly stringent selection of variants with low false positive rate. To further decrease the false positive rate, called variants were manually controlled by excluding those occurring at the 5'or $3^{\prime}$ prime end of the sequence reads and those having an insufficient quality (Phred score less than 25 ). This pipeline was suitable to call single or few bases aberrations (substitutions, small 
insertions and deletions) and gave $0 \%$ false positive as confirmed by additional Sanger analyses (Supplemental Table S4, column 'number validated samples'). Among the previously sequenced (at genetic counseling) pathogenic mutations in MMR genes, 12 out of the 14 familial MMR mutations consisting of few nucleotide alterations were retrieved. These mutations were distributed in 27 subjects and the presence was confirmed in all (see also Table I). Two out of 14 familial MMR mutations consisting of few nucleotide alterations could not be retrieved because located on regions that were poorly covered in the two DNA carrier samples (1204079_30 and 12-04-079_38). Beside these 14 mutations, five additional familial MMR mutations were identified at counseling in six samples (12-04-079_13, 18, $29,23,27$ and 35) but they consisted of relatively large chromosomal rearrangement that could not be retrieved by our pipeline (Table I). In addition, among the variants outside the MMR genes, 19 that were non-deposited in the dbSNP database were selected for Sanger sequencing and their presence was validated in all 21 subjects carrying the variant. Also, the presence of seven identified polymorphisms deposited in dbSNP was validated in the 22 subjects' DNA carrying the polymorphic allele. Sanger sequencing was performed using the BigDye Termination v.1.1. (Life Technologies, Bleiswijk, Netherlands) as recommended. Prior to sequence reactions and analyses, DNA regions were amplified by PCR (Taq polymerase, Fermentas, Burlington, CA, USA) using primers listed in Supplemental Table S4 (forward primers were used for Sanger sequence reactions).

Supplementary data due to many presented columns in Excel processed via download link:

https://www.oncotarget.com/index.php?journal=oncotarget\&page=rt\&op=suppF iles\&path []$=5694 \&$ path []$=18434$

Supplementary Table S1: List of captured genes

Supplementary Table S2: Coverage data per region per sample

Supplementary Table S3: List of all selected variants (outside MMR genes) identified in 35 subjects

Supplementary Table S4: List of primers used for Sanger sequencing analyses 
Chapter 3

\section{Supplemental Figures S1-S6}

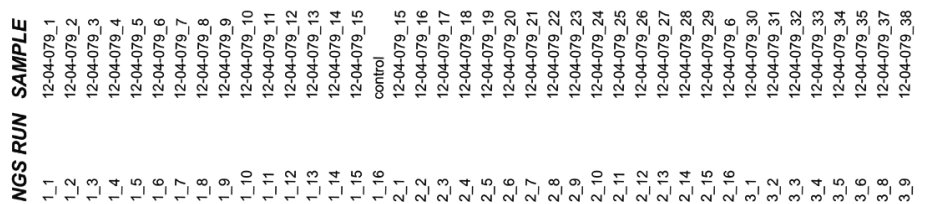

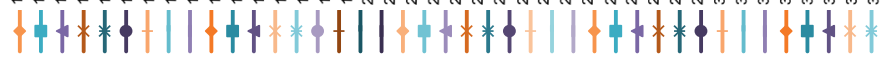

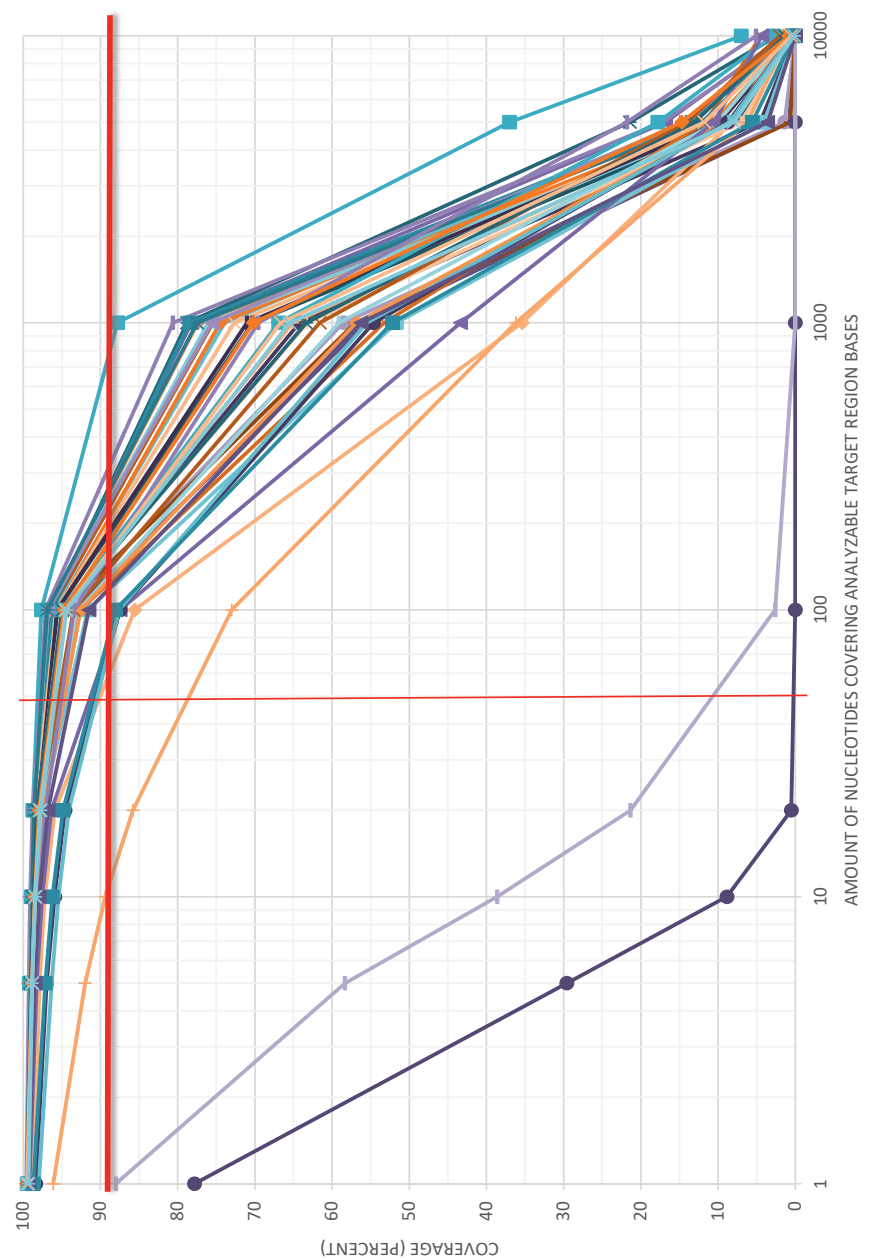

Supplementary Figure S1: Quality control. Quality Control analysis on coverage was performed by using Surecall as described in the main manuscript text. A threshold for read depth was set of at least 50 times (x axes), and a threshold for coverage at $90 \%$ (y axes) of the original regions of interest. The two samples that did not match this criteria cluster clearly separate from the rest (12-04-079_22 and 12-04-079_25). Sample 12-04-079_31 was of borderline sufficient quality. 


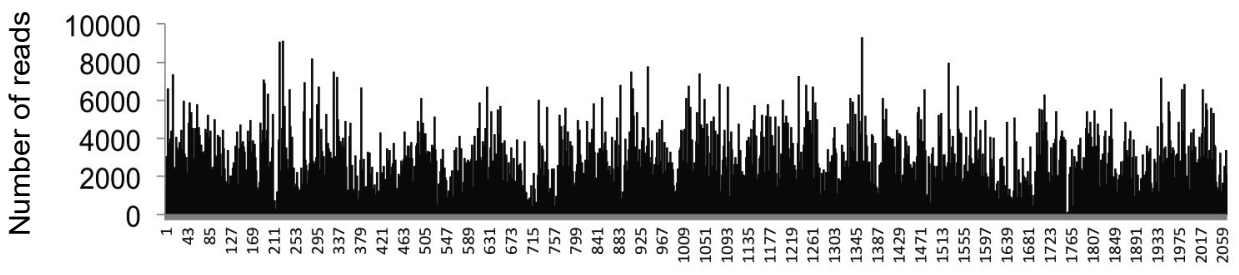

Supplementary Figure S2: Average coverage of the captured regions. Average coverage of the 2069 captured regions covering the 154 genes. The samples with low quality (1204-079_22 and 25) are excluded. Mean reads (plus maximum and minimum) per each region in each sample are given in Supplemental Table S2, which gives also the correspondence between region number (on the $x$ axes) and captured gene exon (with chromosomal coordinates).

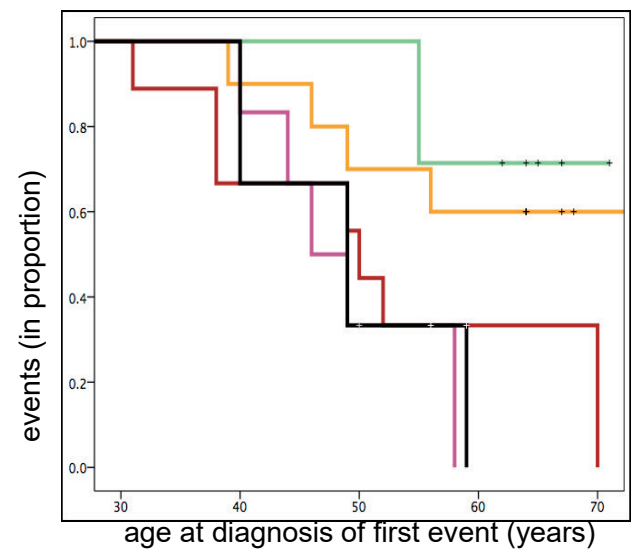

\section{Number of risk-variants beside the MMR familial mutation}

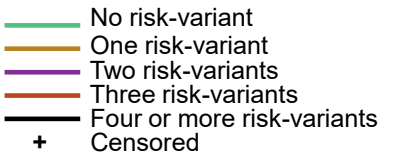

\begin{tabular}{|c|c|c|c|c|c|c|c|c|}
\hline \multirow[t]{2}{*}{ No. risk-variants } & \multirow{2}{*}{$\begin{array}{l}\text { lifespan* } \\
\qquad p=n s\end{array}$} & \multirow{2}{*}{$\begin{array}{c}\text { total } \\
n\end{array}$} & \multicolumn{2}{|c|}{$\begin{array}{c}\text { Any poor- } \\
\text { phenotype event }\end{array}$} & \multicolumn{2}{|c|}{$\begin{array}{l}\text { EC before } 50 \\
\text { years }\end{array}$} & \multicolumn{2}{|c|}{ Second tumour } \\
\hline & & & $n$ & $\%$ & $\mathrm{n}$ & $\%$ & $n$ & $\%$ \\
\hline $\begin{array}{l}0 \\
1\end{array}$ & $\begin{array}{l}66 \pm 5 \\
65 \pm 7\end{array}$ & $\begin{array}{c}7 \\
10\end{array}$ & $\begin{array}{l}3 \\
4\end{array}$ & $\begin{array}{l}42.9 \\
40.0\end{array}$ & $\begin{array}{l}0 \\
3\end{array}$ & $\begin{array}{c}0.0 \\
30.0\end{array}$ & $\begin{array}{l}2 \\
2\end{array}$ & $\begin{array}{l}28.6 \\
20.0\end{array}$ \\
\hline 2 & $65 \pm 7$ & 6 & 5 & 83.3 & 4 & 66.6 & 3 & 50.0 \\
\hline 3 & $64 \pm 12$ & 9 & 7 & 77.8 & 4 & 44.4 & 5 & 55.5 \\
\hline $4-5$ & $64 \pm 23$ & 3 & 3 & 100.0 & 2 & 66.6 & 1 & 33.3 \\
\hline overall & $65 \pm 11$ & 35 & 21 & 60.0 & 13 & 37.1 & 13 & 37.1 \\
\hline Log-Rank & & & \multicolumn{2}{|c|}{0.004} & \multicolumn{2}{|c|}{0.02} & \multicolumn{2}{|c|}{0.08} \\
\hline
\end{tabular}

Supplementary Figure S3: Kaplan-Meier analyses of any poor clinical phenotype event: diagnosis of endometrial cancer below 49 years and/or diagnosis of a second cancer. Kaplan-Meier analysis of the occurrence of any event classified as poor-clinical-phenotype (either diagnosis of endometrial cancer at 49 years or earlier, or the diagnosis of a second tumor other than the endometrial one) among subjects carrying no risk-variants, one, two, three or more than four risk-variants beside the MMR gene familial mutation. Overall comparisons: statistic computed with SPSS; d.f. = degrees of freedom; Sig = value for statistical significance. The table at the bottom shows the overall distributions of the events among the difference categories. * lifespan was extrapolated at 2013. Log-Rank (Mantel-Cox) for statistical significance is shown for the occurrence of any poor phenotype event (shown also as Kaplan-Meier graph, top left), and for the early diagnosis of endometrial cancer or the diagnosis of a second tumor separately. Data in the KaplanMeier graph (top left) show that subjects carrying no or one risk-variant beside the MMR 
mutation cluster separately from subjects carrying two or more risk-variants. In the main text, analyses were performed by categorizing subjects in those carrying no or one riskvariants and those carrying more than two risk-variants beside the MMR mutation.

\begin{tabular}{|c|c|c|c|c|c|c|c|c|c|}
\hline FAMILY & \begin{tabular}{|l|} 
NUMBER \\
METC \\
$12-04-079$ \\
\end{tabular} & $\begin{array}{c}\text { TUMOR } \\
\text { PHENOTYPE }\end{array}$ & $\begin{array}{l}\text { AGE IN } \\
2013\end{array}$ & \begin{tabular}{|c|} 
Familial \\
MMR \\
mutation
\end{tabular} & $\begin{array}{c}\text { Additional MMR } \\
\text { variant }\end{array}$ & total & \multicolumn{3}{|c|}{$\begin{array}{l}\text { Risk-variants outside the } \\
\text { MMR genes }\end{array}$} \\
\hline \multirow{2}{*}{ A } & 2 & $\mathrm{~N}$ & 73 & MSH6 & none & 1 & $\begin{array}{l}\text { NCOR2- } \\
\text { S1964C }\end{array}$ & & \\
\hline & 3 & poor (M) & 71 & MSH6 & none & 3 & \begin{tabular}{|l|} 
NCOR2 \\
S1964C \\
\end{tabular} & $\begin{array}{l}\text { MGMT } \\
\text { P233L }\end{array}$ & $\begin{array}{l}\text { DICER1 } \\
\text { A872T }^{(16}\end{array}$ \\
\hline \multirow{2}{*}{$\mathbf{E}$} & 19 & $N$ & 62 & $\mathrm{MSH} 2$ & rs1800937 & 0 & & & \\
\hline & 17 & $\operatorname{poor}(\mathrm{Y})$ & 58 & $\mathrm{MSH} 2$ & none & 2 & $\begin{array}{l}\text { PPARGC1A } \\
\text { G735V }\end{array}$ & $\begin{array}{l}\text { NOTCH1 } \\
\text { P2448S }\end{array}$ & \\
\hline \multirow{2}{*}{$\mathbf{F}$} & 28 & $N$ & 67 & MSH6 & none & 1 & \begin{tabular}{|l} 
FANCM \\
L57F $^{(1)}$
\end{tabular} & & \\
\hline & 26 & $\operatorname{poor}(\mathrm{Y})$ & 56 & MSH6 & none & 3 & $\begin{array}{l}\text { FANCM } \\
\text { L57F }^{(1)}\end{array}$ & \begin{tabular}{|l} 
EPHB2 \\
C621R $^{(18)}$
\end{tabular} & $\begin{array}{l}\text { MLL } \\
\text { ins3439 }\end{array}$ \\
\hline \multirow{2}{*}{ B } & 6 & N & 56 & MSH6 & none & 3 & $\begin{array}{l}\text { CYP1A1 } \\
\text { C457fs }\end{array}$ & \begin{tabular}{|l|} 
BRCA2 \\
D596H
\end{tabular} & $\begin{array}{l}\text { RET } \\
\text { N361K }\end{array}$ \\
\hline & 7 & poor (M) & 87 & MSH6 & rs 1042821 & 3 & \begin{tabular}{|l} 
CYP1A1 \\
C457fs* \\
\end{tabular} & \begin{tabular}{|l|} 
BRCA2 \\
D596 $\mathrm{H}^{(6)}$
\end{tabular} & $\begin{array}{l}\text { RET } \\
\text { N361K }\end{array}$ \\
\hline \multirow{2}{*}{ C } & 8 & $N$ & 65 & MSH6 & $\begin{array}{l}\text { MSH6-L396V } \\
\text { rs2020908 (cat-2) }\end{array}$ & 0 & & & \\
\hline & 9 & poor (M) & 63 & MSH6 & $\begin{array}{l}\text { MSH6-L396V } \\
\text { rs2020908 (cat-2) }\end{array}$ & 0 & & & \\
\hline \multirow{2}{*}{ G } & 29 & poor $(Y, M)$ & 82 & MLH1 & none & 1 & \begin{tabular}{|l} 
RXFP2 \\
$T_{222 P^{(7)}}$
\end{tabular} & & \\
\hline & 18 & $\operatorname{poor}(\mathrm{Y})$ & 56 & MLH1 & rs12998837 & 2 & $\begin{array}{l}\text { RAD51D } \\
\text { D90G }^{(11)}\end{array}$ & $\begin{array}{l}\text { NTRK1 } \\
\text { T300A }\end{array}$ & \\
\hline \multirow{3}{*}{ D } & 12 & poor $(\mathrm{Y})$ & 70 & $\mathrm{MSH} 2$ & none & 1 & $\begin{array}{l}\text { COMT } \\
\text { R128H }\end{array}$ & & \\
\hline & 11 & $\operatorname{poor}(\mathrm{Y})$ & 61 & $\mathrm{MSH} 2$ & none & 3 & $\begin{array}{l}\text { COMT } \\
\text { R128H }\end{array}$ & $\begin{array}{l}\text { ATM } \\
\text { L1590F }^{(14)}\end{array}$ & $\begin{array}{l}\text { TSC1 } \\
\text { R190C }\end{array}$ \\
\hline & 16 & poor $(\mathrm{Y}, \mathrm{M})$ & 67 & $\mathrm{MSH} 2$ & rs 1042821 & 3 & \begin{tabular}{|l} 
APC \\
Y1624S
\end{tabular} & $\begin{array}{l}\text { BARD1 } \\
\text { S761N }\end{array}$ & $\begin{array}{l}\text { ATM } \\
\text { L1590F }^{112}\end{array}$ \\
\hline
\end{tabular}

Supplementary Figure S4: Clinical phenotypes and genetic characterization of relatives. The seven families ( 15 subjects) for which DNA from relatives was screened. Among the five families in which members have distinct cancer phonotypes ( $A, E, F, B$ and $C)$, in three $(A, E$ and $F)$, the member with poor clinical phenotype carries more risk-variants than the one with neutral phenotype. In Family $B$, both members carry three risk-variants and the woman with neutral phenotype is relatively young compared to her relative, characterize by a poor cancer phenotype. Members if family $C$ (one poor and neutral) carry no risk-variants. The remaining two families comprise women who had all a poor cancer phenotype. All members of these families carries at least one risk-variant. Superscripts refer to dbSNP accession, when applicable: (1) rs142007602; (6) rs56328701; (7) rs121918303; (11) rs180869630;(14) rs35962982; (15) rs118203400; (16) rs149242330; (18) rs149475426; (19) rs142155101. 
A. EXPRESSION OF ER- $\alpha$ and Phospho-Ser-167-ER- $\alpha$

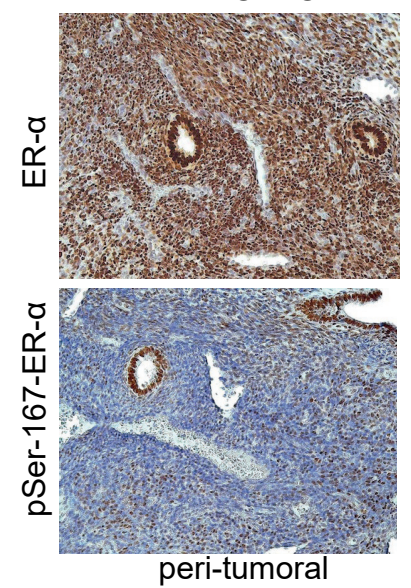

B.

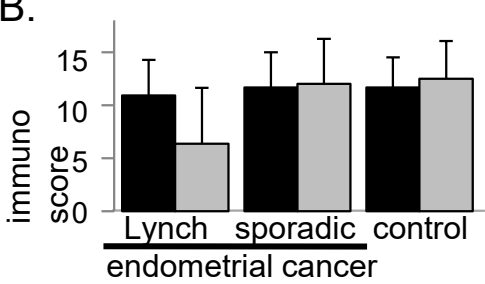

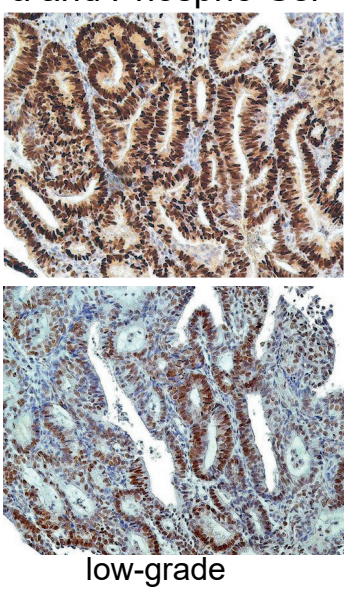

low-grade
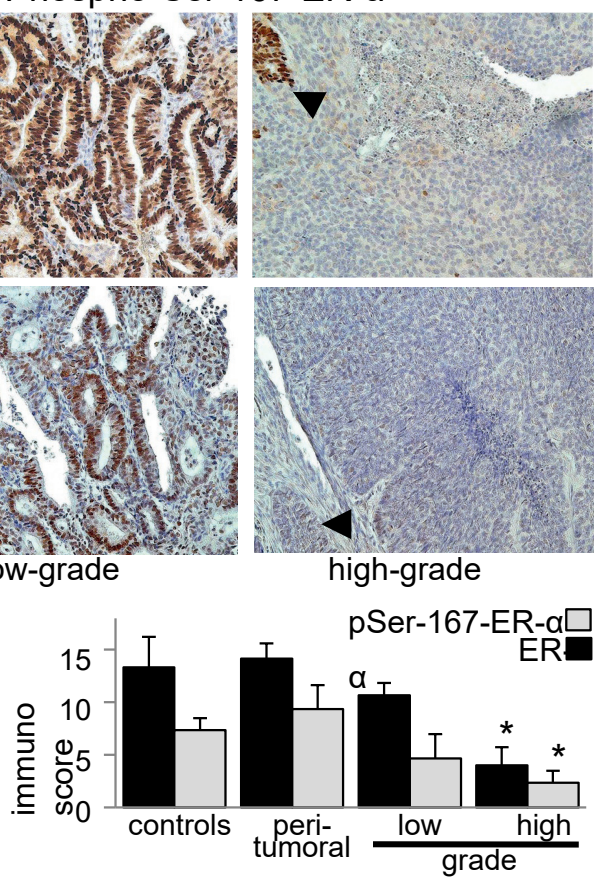

C. EXPRESSION OF COMT, NCOR2, CYP1A1, RET, IGF1R, mTOR COMT

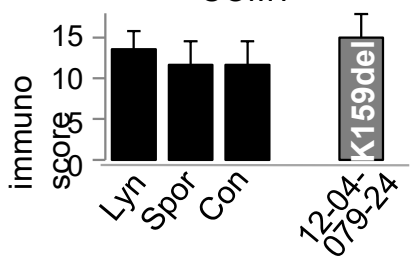

NCOR2

CYP1A1

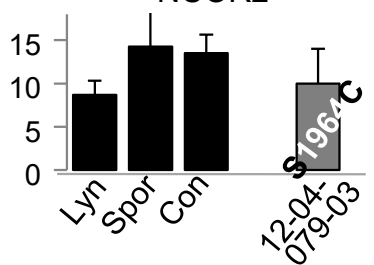

IGF1R

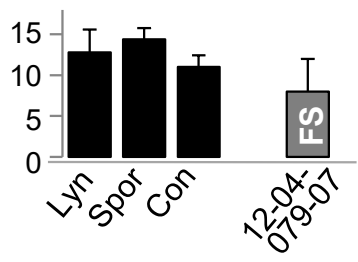

$\operatorname{mTOR}(\&$ signalling)
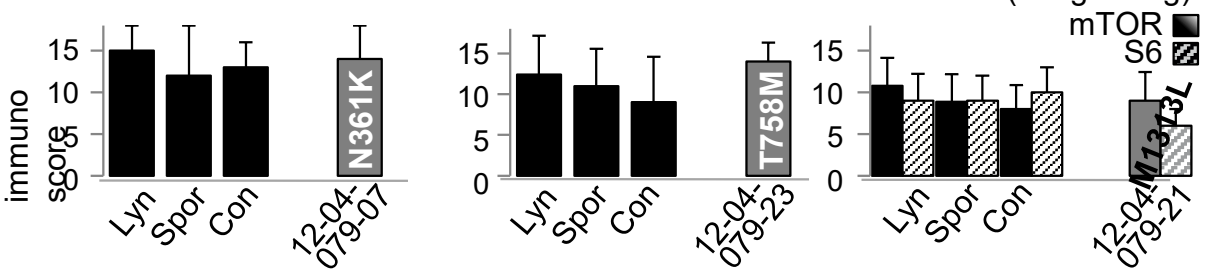

Supplementary Figure S5: Estrogen signaling and protein expression in tumor specimens. A. Representative immunohistochemical images showing expression of the estrogen receptor (ER-a) and the phosphorylated form at Ser-167 in normal endometrium adjacent to tumor tissues (peri-tumoral), low-grade and high-grade Lynch syndrome endometrial cancer. ' $s$ ' marks the stromal component, ' $e$ ' the epithelial glandular cells. Stroma is abundant in post-menopausal endometrium and decreases in cancer. In high-grade lesions the cytological differentiation between stromal and epithelial cells is less evident. 
Red-arrowheads on the images at the right indicate one area of low-grade cancer with clearly well-differentiated epithelial cells. Bar $=100 \mu \mathrm{m}$. B. Quantification of ER-a (black) and phosphoSer-167-ER-a (grey) in Lynch syndrome endometrial cancer $(n=8)$, sporadic endometrial cancer $(n=7)$ and post-menopausal controls $(n=5)$. Immunoscores range: 0-15. Mean values \pm standard deviations (SD) are shown. C. Quantification (range 0-15) of the immunohistochemical expression of COMT, NCOR2, CYP1A1, RET, IGF1R, mTOR. Expression levels are shown for the group of Lynch syndrome tumors, the group of sporadic tumors, the controls (black bars) and the specimen bearing the indicated risk-variant (grey bars). Black bars are the mean values \pm SD of the different subjects (Lynch syndrome specimens without the variant, $\mathrm{n}=7$; sporadic cancers, $\mathrm{n}=$ 7; controls, $n=5$ ). Grey bars are the mean values \pm SD of four independent areas of the tumor from the indicated subject bearing the risk-variant.

\section{EXPRESSION OF CYP1A1}
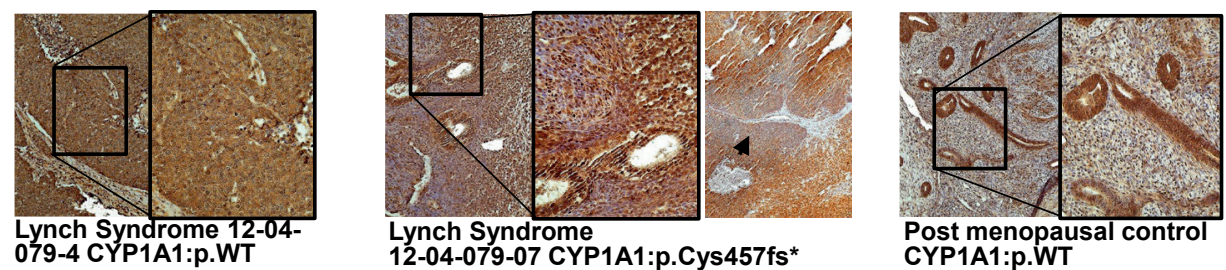

\section{EXPRESSION OF COMT}

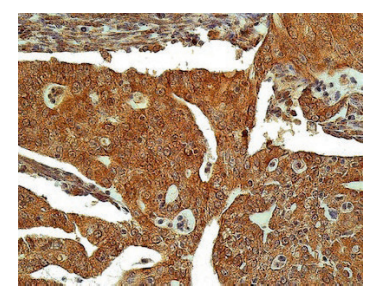

Lynch Syndrome 12-04-07904 COMT:p.WT
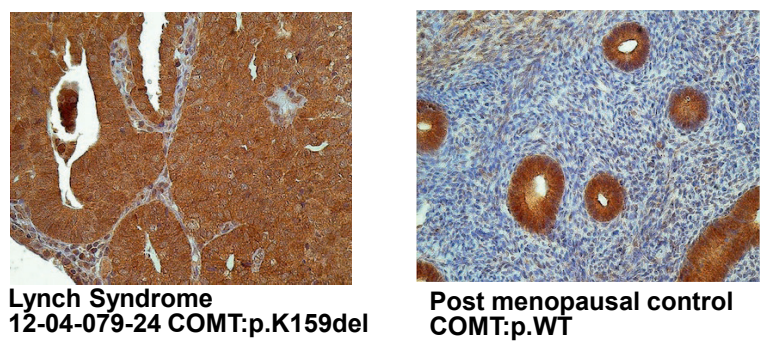


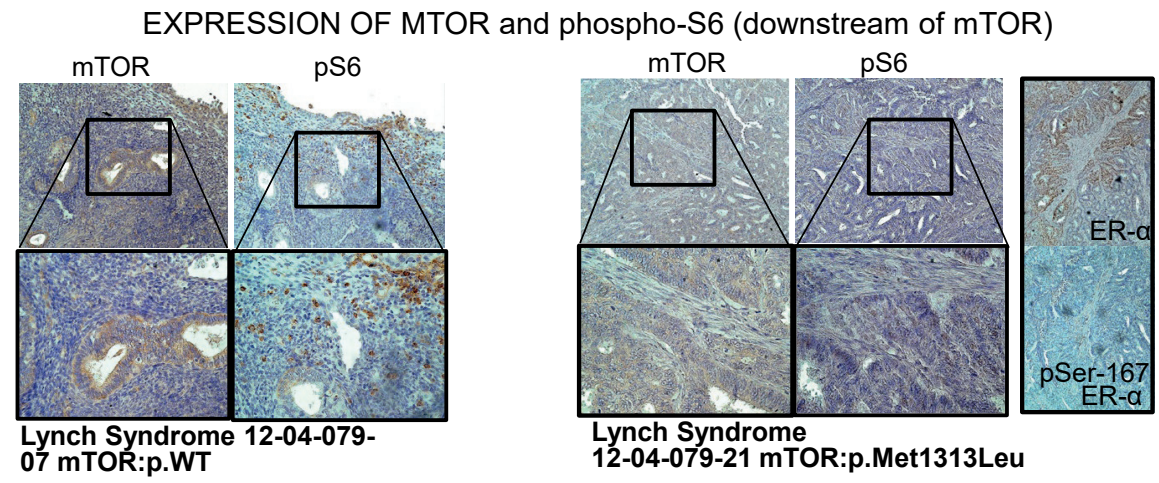

\section{EXPRESSION OF NCOR2}
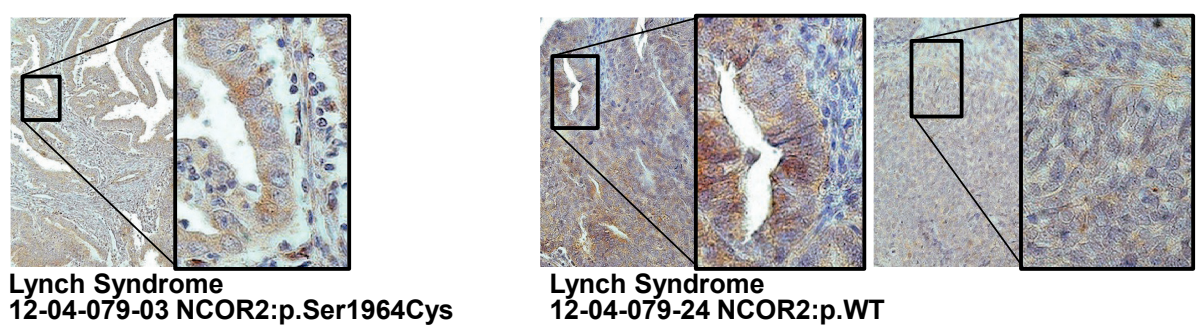

Lynch Syndrome

12-04-079-24 NCOR2:p.WT

Supplementary Figure S6: Analysis of tumor material by immunohistochemistry. Immunohistochemical stainings for CYP1A1, COMT, MTOR (and downstream signal protein S6) and NCOR2. Overall, immunohistochemical analysis (including those for RET and IGF1R in Figure 2 and Supplemental figure 5) shows high expression levels of these proteins (and the downstream signaling activation in case of mTOR) in the endometrium and in endometrial cancer, proofing that they play a role in endometrial physiology. A. (top series). Immunohistochemical images of a tumor and a normal endometrium specimens bearing the wild type CYP1A1 or the tumor with risk-variant CYP1A1:p.Cys457fr*, showing the expression of the protein CYP1A1. (bottom series). Immunohistochemical images of a tumor and a normal endometrium specimens bearing the wild type COMT or the risk-variant COMT:p.Lys159del, showing the expression of the protein COMT 


$$
\text { II }
$$


Part II

Unbiased Exome 
CHAPTER 4

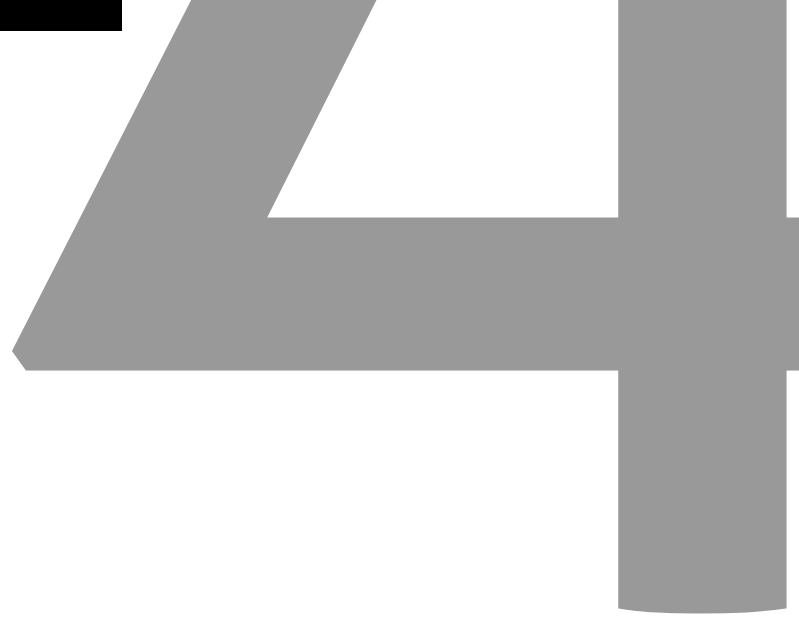




\section{Exome Sequencing Reveals a Novel Moroccan Founder Mutation in SLC19A3 as a New Cause of Early- Childhood Fatal Leigh Syndrome}

Mike Gerards, Rick Kamps, Jo Vanoevelen, Iris Boesten, Eveline Jongen, Bart de Koning, Hans R. Scholte, Isabel de Angst, Kees Schoonderwoerd, Abdelaziz Sefiani, Ilham Ratbi, Wouter Coppieters, Latifa Karim, René de Coo*, Bianca van den Bosch*, Hubert Smeets*

* These authors contributed equally to this work 


\section{Abstract}

Leigh syndrome is an early onset, often fatal progressive neurodegenerative disorder caused by mutations in the mitochondrial or nuclear DNA. Until now, mutations in more than 35 genes have been reported to cause Leigh syndrome, indicating an extreme genetic heterogeneity for this disorder, but still only explaining part of the cases. The possibility of whole exome sequencing enables not only mutation detection in known candidate genes, but also the identification of new genes associated with Leigh syndrome in small families and isolated cases. Exome sequencing was combined with homozygosity mapping to identify the genetic defect in a Moroccan family with fatal Leigh syndrome in early childhood and specific MRI abnormalities in the brain. We detected a homozygous nonsense mutation (c.20C>A; p.Ser7Ter) in the thiamine transporter SLC19A3. In vivo overexpression of wild-type SLC19A3 showed an increased thiamine uptake, whereas overexpression of mutant SLC19A3 did not, confirming that the mutations results in an absent or non-functional protein. Seventeen additional patients with Leigh syndrome were screened for mutations in SLC19A3 using conventional Sanger sequencing. Two unrelated patients, both from Moroccan origin and one from consanguineous parents, were homozygous for the same p.Ser7Ter mutation. One of these patients showed the same MRI abnormalities as the patients from the first family. Strikingly, patients receiving thiamine had an improved life-expectancy. One patient in the third family deteriorated upon interruption of the thiamine treatment and recovered after reinitiating. Although unrelated, all patients came from the province Al Hoceima in Northern Morocco. Based on the recombination events the mutation was estimated to have occurred 1250-1750 years ago. Our data shows that SLC19A3 is a new candidate for mutation screening in patients with Leigh syndrome, who might benefit from high doses of thiamine. Especially Moroccan Leigh patients should be tested for the c.20C >A founder mutation in SLC19A3. 


\section{Introduction}

Leigh syndrome (MIM256000) is a progressive neurodegenerative disorder characterized by focal, bilateral lesions in one or more areas of the central nervous system, including basal ganglia, thalamus, cerebellum, brainstem and spinal cord. Leigh syndrome is an early onset mitochondrial disorder with extensive genetic heterogeneity and can be caused by mutations in the mitochondrial DNA (mtDNA) and many nuclear genes. The most common cause of Leigh syndrome is a defect in one of the subunits or assembly factors of the oxidative phosphorylation complexes (complexes I, II, IV, V and coenzyme Q) [1]. Additionally, mutations in components of the pyruvate dehydrogenase complex and in the gene encoding the leucine-rich PPR motif containing protein have been described [2-4]. In total, mutations in at least 35 genes have been reported to cause Leigh syndrome. Although for example $25-75 \%$ of Leigh patients with complex IV deficiency harbour mutations in the complex IV assembly factor SURF1 [5], no clear candidate gene can be selected in the majority of patients despite small phenotypic differences. Because of the extreme genetic heterogeneity of this disorder, gene specific mutation detection is a time consuming and expensive approach. Additionally, the limited genotype-phenotype correlations, the small size of families and large number of isolated cases make classical family based approaches, like linkage analysis, less applicable. Recently, new sequencing techniques have been developed enabling the sequencing of large packages of candidate genes, the whole protein-coding region (exome) or even the whole genome at higher rate and at lower costs [6]. Especially exome sequencing has become a valuable tool for identifying the genetic cause for different disorders. Over the last 3 years, exome sequencing enabled the identification of over 30 new genes involved in Mendelian diseases [7].

Here we describe a new candidate gene for Leigh syndrome identified by whole exome sequencing in combination with homozygosity mapping in a consanguineous Moroccan family with Leigh syndrome and specific abnormalities on MRI. 


\section{Patients and Methods}

\section{Patients}

Family $a$ is a Moroccan family with three healthy children and three deceased children, due to Leigh syndrome, born from healthy consanguineous parents (Figure 1). The deceased children died 1 month after birth due to respiratory insufficiency. Patient II: 1 was born at 41 weeks gestation without complications. He was admitted to the hospital for his opisthotonic posture, inconsolable crying and roving eye movements after 1 month of age. After 2 to 3 weeks he began experiencing seizures. To reduce the seizures, phenobarbital was prescribed. He was a hypotonic boy with hyperreflexia in the extremities, including positive Babinski sign. Later he developed feeding difficulties. His blood tests showed a slightly increased lactic acid of $3.8 \mathrm{mmol} / \mathrm{I}$ and an alanine level which was in the upper normal range. Urine culture revealed a hemolytic streptococcus group B. Biochemical analysis of respiratory chain complex activities in fibroblasts was normal. His brain CT scan demonstrated various hypodense areas, most pronounced in the basal ganglia. After 1 month and 7 days he died of Leigh syndrome, mainly due to apneas and Cheyne-Stokes type of breathing. The fifth child of this family (patient II:5) was born at a gestational age of 40 weeks. After almost 5 weeks of age he presented with convulsions, nystagmus, hypotonia with positive Babinski sign and sun-set phenomenon. His convulsions responded well to phenobarbital. Soon after presentation a brain MRI was performed, which demonstrated abnormal signal intensity in the basal ganglia, thalamus, brainstem and cerebellum (Figure 2a). Brain MRS showed a lactate peak in the basal ganglia. These findings were similar to the clinical course of his brother and therefore carnitine, riboflavin and vitamin $\mathrm{E}$ were prescribed. Blood tests revealed disturbance of liver function with a gamma GT of $114 \mathrm{U} / \mathrm{I}$, ASAT $109 \mathrm{U} / \mathrm{I}$, ALAT 70 $\mathrm{U} / \mathrm{I}$, an increased lactic acid of $4.4 \mathrm{mmol} / \mathrm{I}$ and pyruvate of $247 \mu \mathrm{mol} / \mathrm{l}$. In cerebrospinal fluid increased levels of leucine and isoleucine were found. Biochemical analysis on muscle and skin biopsy showed no clear decreased activity of the respiratory chain complexes. After 1 month of age he died of respiratory failure. The youngest son (patient II:6) was born at a gestational age of 41 weeks without complications and showed an identical disease course as his brothers. He was first admitted to the hospital at 3 weeks of age with feeding 
difficulties, opisthotonic posture and roving eye movements. Clinical examination showed hypotonic extremities and symmetric hyperreflexia with positive Babinski sign. Blood tests revealed a slightly increased lactic acid of $3.4 \mathrm{mmol} / \mathrm{l}$. Neurotransmitters in cerebrospinal fluid showed lowered levels of HVA and HIAA. Brain specific proteins MBP, GFAP and fosfo-tau were increased in liquor. Metabolic urine tests showed slightly increased levels 2-ketoglutaric acid, 2-hydroxy-glutaric acid, glutaric acid, succinate and 2-ketoadipic acid. Biochemical analysis on skin biopsy revealed a slightly decreased activity of complex IV. Later he began experiencing breathing difficulties. Treatment was started with carnitine, vitamin $\mathrm{E}$, riboflavin and $\mathrm{CoQ}_{10}$. The patient died due to a progressive abnormal breathing pattern at 6 weeks of age.

Family $b$ is a consanguineous Moroccan family with seven children (Figure 1) of which two girls died after 4 weeks of age due to a progressive neurologic deterioration caused by Leigh syndrome. When patient II: 1 was admitted to the hospital, she was irritable, cried inconsolably and presented with jitteriness. She had drinking difficulties and frequent green defecation. Her extremities were hypertonic with clonic triceps surae reflexes including a positive Babinski sign. Shortly after hospitalization she became respiratory insufficient with progressive neurological symptoms. Her EEG was normal. Her brain CT scan showed symmetric hypodense areas in pons, mesencephalon, vermis and thalamus. Medication existed of phenobarbital. Before she died, she was hypotonic with hyperreflexia in the extremities. Patient II:5 had sucking difficulties and could not open her eyes for 3 days. Because of the progressive disease course of her older sister, she was admitted to the hospital for observation. At presentation she had an abnormal breathing pattern and increasing convulsions confirmed by EEG. The epileptic activity was treated with phenytoin. Clinical examination showed hyperreflexia in the extremities including a positive Babinski sign. Apart from that, the extremities were hypertonic with an axial hypotonia. Lactic acid was found slightly increased in liquor as well as in blood. Her MRI demonstrated abnormal signal intensity in basal ganglia, thalami, mesencephalon and brainstem. The lesions were bilateral and symmetrical. After 1 month her clinical condition deteriorated rapidly. She began experiencing a Cheyne-Stokes respiration, which eventually led to her death. 


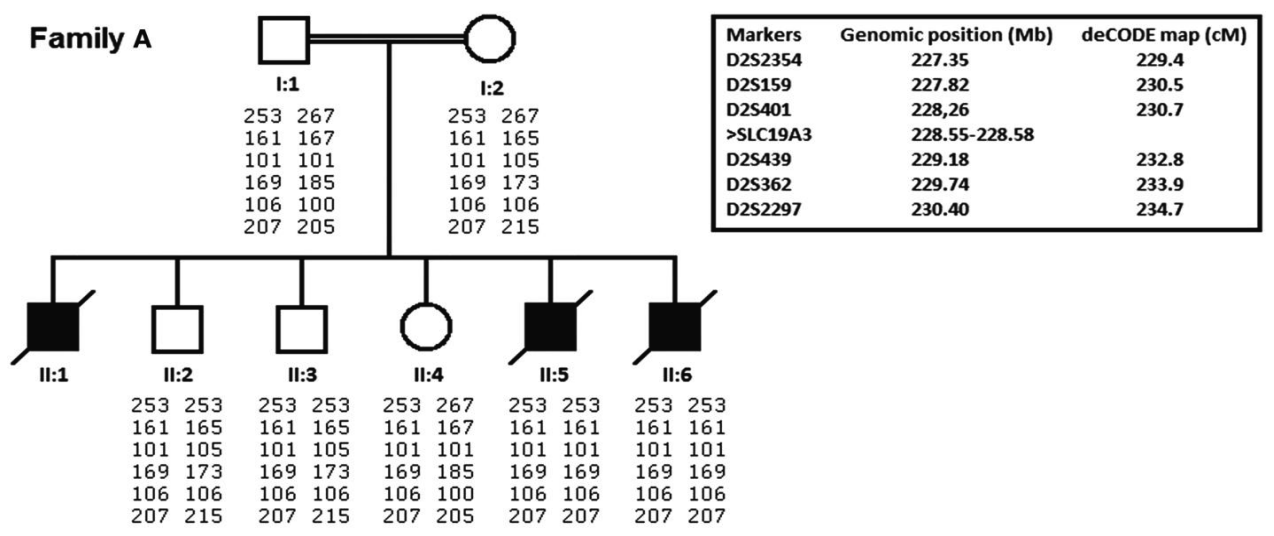

Family B

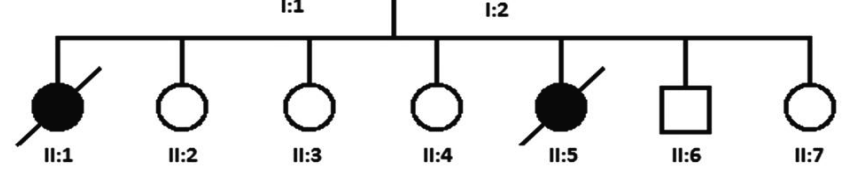

$253 \quad 271$

161161

101101

169169

106106

207207

Family C

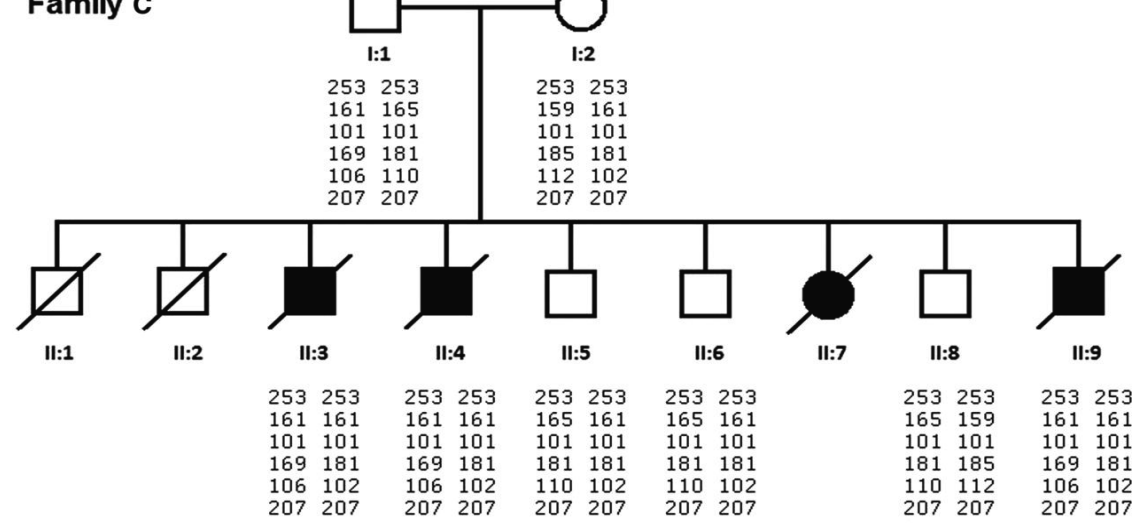

Figure 1. Pedigrees of 3 Moroccan families with Leigh syndrome showing the haplotype in the region surrounding the mutation 
Moroccan family c consists of nine children, of which six died due to a Leigh-like syndrome (Figure 1). All children were born to healthy non-consanguineous parents. The children who were clinically investigated had a very similar disease course and features. Three of the children died at 1 month of age, one at three years, one at fifteen years and one at 20 years of age.

The proband, patient II: 3, was born at a gestational age of 39 3/7 weeks. Besides meconium in the amniotic fluid there were no further complications. Because his brother died of a progressive encephalopathy he was hospitalized for observation during the first month of live. At 1 month of age he appeared to be alternating hypo- and hypertonic and irritable. His clinical picture and failure to thrive posed the possibility of a mitochondrial disease. Oxidative phosphorylation was measured in a fresh muscle biopsy of the patient. Polarographic measurements were performed in isolated muscle mitochondria by a Clarke electrode as previously described [8] with pyruvate + malate, succinate + rotenone and ascorbate + TMPD. Oxygen consumption in the isolated mitochondria of the patient with pyruvate as the substrate was only $12 \%$ of normal oxygen consumption and with succinate and ascorbate as substrate $50 \%$ of normal oxygen consumption. When normalized for citrate synthetase, oxygen consumption with pyruvate was $25 \%$ compared to normal and the oxygen consumption with succinate and ascorbate was normal. Biochemical measurement of Complex I and IV activities in the muscle homogenate were normal. Almost the same biochemical result was found in proband II:7 with normal activities of the respiratory chain complexes and a lowered oxygen consumption of $25 \%$ compared to normal with pyruvate + malate. The activities of pyruvate dehydrogenase and a-ketoglutarate dehydrogenase measured in the muscle homogenate in vitro, after addition of thiamine pyrophosphate, were increased respectively to $441 \%$ and $156 \%$ compared to controls. In the 1990 's treatment with vitamin cocktails for mitochondrial disease was recommended. The boy (II:3) was treated with thiamine and riboflavin, which were later discontinued because he was developing well. Shortly after this change of treatment regimen he became hyperactive and irritable again, so treatment with these vitamins was resumed and the irritability disappeared. The following year he experienced three periods with relapses presented with an opisthotonic posture, inconsolable crying, and 
loss of motor functions. His brain CT scan showed hypo-intense lesions in the thalami and basal ganglia, suggestive for Leigh disease. A metabolic analysis for a wide range of metabolic disorders was normal. Biotin was added to his standard medication. At the age of eleven, he presented with a status epilepticus for which he was treated with sodium valproate and phenytoin. When he was twelve, he was severely mentally retarded. In that same year a gastrostomy was performed because of swallowing difficulties. The patient eventually died due to respiratory insufficiency and an abnormal breathing pattern at the age of 20 . Patient II: 9 was born at a gestational age of 42 weeks without complications. Soon after birth, treatment was started with riboflavin, thiamine and carnitine. At 1 month of age he became hypertonic and cried inconsolably. After one year he presented with seizures. His EEG demonstrated a severely distorted picture with epileptogenic phenomena. Therefore he received phenobarbital combined with carbamazepine. At six years of age severe psychomotor retardation was confirmed and he began experiencing reflux and spasticity. Brain MRI at six years of age revealed abnormal intensities in the putamen, thalami, nucleus dentate, and to a lesser extent in the globus pallidus (Figure $\mathbf{2 b}$ ). At the age of eight, a percutaneous endoscopic gastrostomy was performed because of his feeding difficulties. A few years later, at the age of eleven, he strongly deteriorated and could hardly make any contact with his environment. The following years he had recurrent respiratory tract infections. The performed muscle biopsy showed COX negative fibres. Biochemical measurements of the OXPHOS complexes in patient II:9 revealed no enzyme deficiency and the oxygen consumption measured with glutamate or succinate as substrate was normal. The many respiratory tract infections proved fatal and he died at the age of fifteen.

\section{Control Population}

Blood samples were collected from 230 unrelated newborns' umbilical cords. They originated from different regions of Morocco and the Moroccan origin of their parents and grandparents was confirmed. Informed consent for DNA analysis was obtained from the parents. Genomic DNA was extracted from $3 \mathrm{ml}$ blood using the salting-out method. 


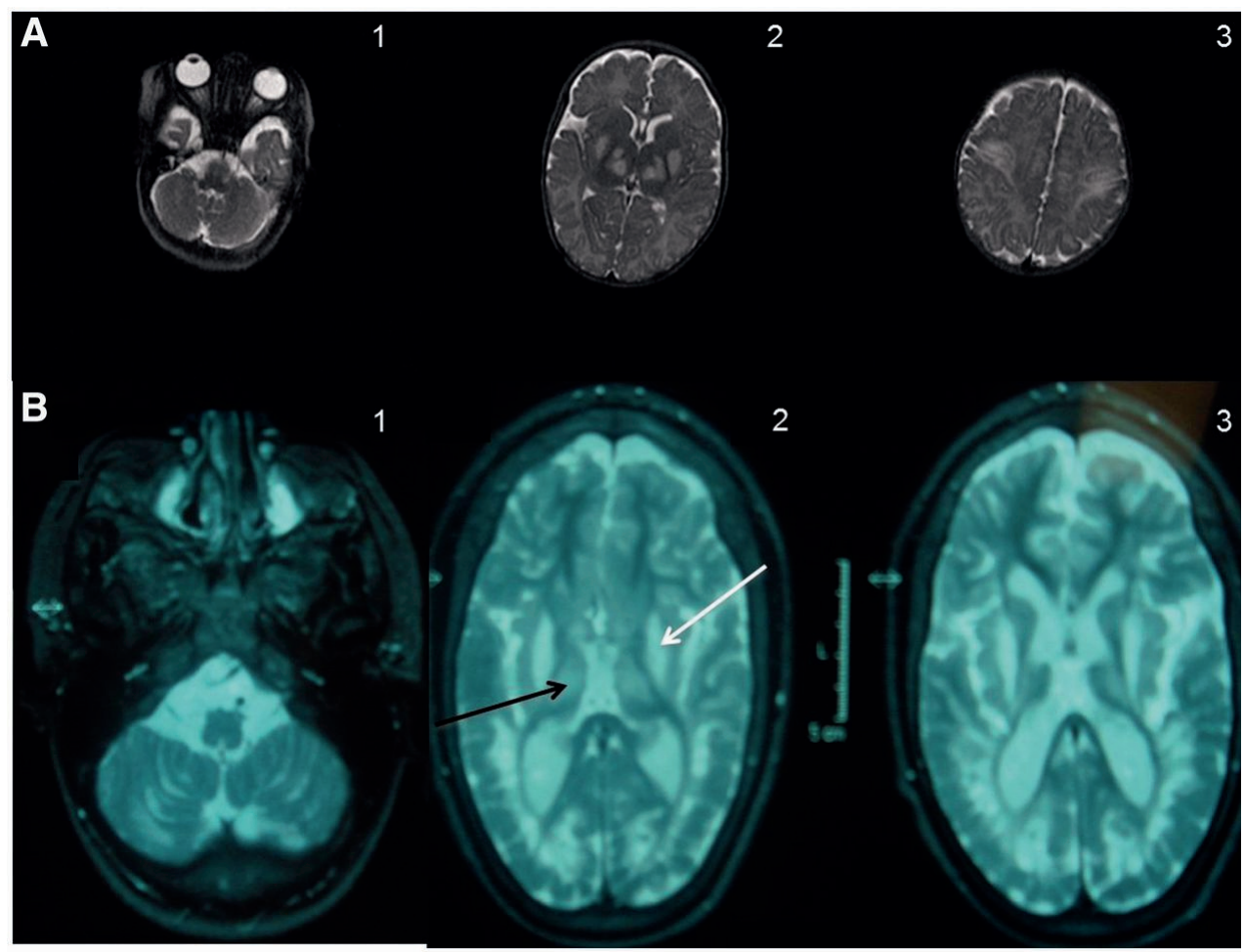

Figure 2. Brain MRIs (T2 weighted images, axial view). a) Upper row, patient II-5 of family an at age 1 month. Image B1 Cerebellum and brain stem show minor changes. B2 Increased signal intensities in putamen and mediocaudal and ventrolateral thalamic nuclei. The caudate nuclei are spared. Image B3 shows an abnormal intensity and swelling of the rolandic area. b) Lower row, patient II-9 of family $c$ at the age of 6 years. A1 Infratentorial increased signal intensities in the periaquaductal and cerebellar region. Image A2 and A3: Supratentorial loss of white matter increasing in antero-posterior direction with subcortical cysts. Relatively sparing of directly periventricular situated white matter. Thin corpus callosum. Pathologic hyperintensity of the putamen (white arrow) and less so of the globus pallidi and thalamic nuclei (black arrow).

\section{Methods}

\section{Homozygosity Mapping}

Homozygosity mapping was performed with the Affymetrix GeneChip Human Mapping $250 \mathrm{~K}$ NspI SNP array using peripheral blood DNA of patients II:5 and II:6 from family a. The DNA was processed and labelled according to the manufacturer's instructions. Genotypes were generated by the Affymetrix GeneChip Genotyping Analysis Software (GTYPE). Homozygous regions were determined by the online tool 'HomozygosityMapper' [9]. 


\section{Whole Exome Sequencing and Analysis}

The exome was captured for patient II: 6 of family a according to the TruSeq Whole Exome Enrichment for Paired-end Sequencing Preparation Guide (Illumina). In short, $1 \mu \mathrm{g}$ of genomic DNA was fragmented using a Covaris S2, with settings optimal for an average fragment peak size of 200-300bp. Fragments were endrepaired, ligated to specific adapters with a single index after the addition of a 3'A. Subsequently the fragments were size selected for approximately 400bp on agarose gel and pre-PCR amplified (10 cycles) to yield a total amount of 500ng for the hybridization step. The DNA library was mixed with the capture probes of the targeted exome regions. The hybridization steps were performed as follows $95^{\circ} \mathrm{C}$ for 10 minutes, 18 cycles of $93^{\circ} \mathrm{C}$ for 1 minute, decreasing $2^{\circ} \mathrm{C}$ per cycle, and $58^{\circ} \mathrm{C}$ for $16-20 \mathrm{hrs}$. Streptavidin affinity was performed to capture targeted regions of interest. Additionally three washes were applied to remove non-specific binding from the beads. Next, the enriched library was eluted from the beads. A second round of hybridization and subsequent washing was done to increase enrichment of the targeted regions. A post-PCR (10 cycles) was performed to amplify the enriched DNA library. Quantification and quality determination of the library was done on a 2100 Bioanalyzer using the High Sensitivity DNA Kit (Agilent). Clusters were generated using the cluster generation kit V5.0 according to the manufacturers protocol (Illumina) and the paired-end library was sequenced using $2 \times 150$ cycles V5.0 paired-end by sequencing by synthesis (SBS) on a Genome Analyzer IIx system (Illumina).

Basecalling was done using Casava 1.6 (Illumina). Sequence reads were aligned against the human reference genome (UCSC hg19) using BWA 0.5.9. Single base variants were identified using samtools 0.1 .16 and annotated using an in house developed annotation python/R script that matched variants to the RefGene and dbSNP132 hg19 tracks from the UCSC genome browser. The data obtained was filtered for genes containing at least two alleles with a nonsynonymous variant or variant leading to premature stop codons, which had an allele frequency of $<0.01$ or were absent in dbSNP132 and the 1000genomes data (1000 Genome Project Consortium, 2010) and present in the homozygous candidate regions. 


\section{SLC19A3 Sequencing and Mutation Specific Restriction Assay}

Specific intronic primers were designed for amplifying the exons and flanking introns of SLC19A3 (Supplementary table 1). PCR was performed in a $10 \mu \mathrm{l}$ reaction volume using Amplitaq Gold Master Mix (manufacturer) with $8 \%$ glycerol, $2 \mathrm{pmol}$ of each primer and $5 \mathrm{ng}$ DNA. The cycle conditions were: $96^{\circ} \mathrm{C}$ for $5 \mathrm{~min}$, followed by 33 cycles of $94^{\circ} \mathrm{C}$ for 30 seconds, $60^{\circ} \mathrm{C}$ for 45 seconds, and $72^{\circ} \mathrm{C}$ for 45 seconds with a final elongation step of $72^{\circ} \mathrm{C}$ for 10 minutes. PCR products were directly sequenced with the PRISM Ready Reaction Sequencing Kit (Perkin-Elmer Life Sciences) on an ABI3730 DNA Analyzer (Applied Biosystems). A mutationspecific restriction assay was developed to screen controls for the c.20A>G mutation. A $5 \mu \mathrm{l}$ PCR product of exon 2 was digested with MseI for 2.5 hours at $37^{\circ} \mathrm{C}$. The mutation results in the gain of a restriction site presenting a fragment of $138 \mathrm{bp}$ instead of $187 \mathrm{bp}$, which was visualized on an agarose gel.

\section{Defining the SLC193A Mutation Haplotype}

All patients and relatives and 230 Moroccan controls were genotyped for six polymorphic markers (D2S2354, D2S159, D2S401, D2S439, D2S362 and D2S2297) flanking the SLC19A3 gene. Primers were fluorescently labelled. Amplicons were amplified in a $50 \mu$ reaction volume using Bioline Taq polymerase. The cycle conditions were: $94^{\circ} \mathrm{C}$ for $5 \mathrm{~min}$, followed by 33 cycles of $94^{\circ} \mathrm{C}$ for 1 $\min , 58^{\circ} \mathrm{C}$ for $1 \mathrm{~min}$, and $72^{\circ} \mathrm{C}$ for $1 \mathrm{~min} 30$ seconds with a final elongation step of $72^{\circ} \mathrm{C}$ for 7 minutes. Subsequently the size of the amplicons was determined on an ABI3730 DNA Analyzer (Applied Biosystems) according to manufacturer's instructions. Analysis was conducted using the Peak Scanner ${ }^{\mathrm{TM}}$ Software 1.0 (Applied Biosystems). The following formula was used to assess the age of the c. $20 \mathrm{C}>\mathrm{A}$ mutation in generations $(G): G=\log \delta / \log (1-\theta)$, with $\delta=\mathrm{LD}=(P d$ $P n) /(1-P n)$. The measure $\delta$ refers to the linkage disequilibrium (LD) between the mutation and the nearest recombinant markers, in our case, D2S2354 and D2S439 (Figure 1). $P d$ is the frequency of the ancestral STR allele among chromosomes carrying the SLC19A3 mutation and $P n$ is the frequency of the ancestral STR allele among chromosomes not carrying the mutation. $\theta$ refers to the recombination fraction between marker and genes [10]. 


\section{Thiamine Transport Assay}

An expression construct containing the SLC19A3 open reading frame was obtained from Open Biosystems (SLC19A3 in PCMV-SPORT6). The mutation was introduced using site-directed mutagenesis with forward primer (5'GATTGTTACAGAACTTAACTAAGCAGTTCCTGG-3' and reverse primer 5'CCAGGAACTGCTTAGTTAAGTTCTGTAACAATCC-3'). The presence of the mutation was verified by sequencing. HEK293 cells were cultured in DMEM containing 10\% Fetal Calf Serum at $37^{\circ} \mathrm{C}$. For overexpression experiments, cells were seeded in 6-well plates and transfected using Lipofectamine 2000 with expression constructs containing either wild-type or mutant SLC19A3 or an empty vector. The transfection efficiency was checked by qPCR of SLC19A3 RNA and SLC19A2 RNA of the wild-type and mutant transfected cell lines. Both the mutant and wild-type SLC19A3 were highly expressed (data not shown). Transfected HEK293 cells were incubated with different concentrations of ${ }^{3} \mathrm{H}$-thiamine in Krebs Ringer Buffer (KRB; $133 \mathrm{mM} \mathrm{NaCl}, 4.93 \mathrm{mM} \mathrm{KCl}, 1.23 \mathrm{mM} \mathrm{MgSO}_{4}, 0.85 \mathrm{mM} \mathrm{CaCl}$, $5 \mathrm{mM}$ glucose, 5 mM glutamine, 10 mM HEPES, 10 mMMES pH7.4) for 20 minutes at $37^{\circ} \mathrm{C}$. Low concentration of radiolabeled thiamine $(15 \mathrm{nM})$ was used to favour high-affinity, low capacity uptake, whereas a high concentration (10 $\mu \mathrm{M})$ was used to favour low-affinity, high capacity uptake. The reaction was stopped with icecold $\mathrm{KRB}$ and samples were dissolved in $0,5 \mathrm{M} \mathrm{NaOH}$ and neutralized with $0,5 \mathrm{M}$ $\mathrm{HCl}$. A fraction of the sample was used for protein quantitation and the remainder was subjected to liquid-scintillation counting. Non-specific uptake was determined by repeating the experiment at $0^{\circ} \mathrm{C}$ and subtracted from the results. The results are calculated as pmol or fmol thiamine uptake/min $\mathrm{mg}$ protein.

\section{Results}

Homozygosity mapping was performed for patients II:5 and II:6 (family a) with the Affymetrix GeneChip Human Mapping 250K NspI SNP array. Both patients were homozygous for an overlapping region on chromosome 2 (220-234Mb) and chromosome 13 (80-101Mb). In these regions a total of 258 genes were present, but no obvious candidate gene. As a next step for identifying the genetic defect, the whole exome of patient II: 6 was sequenced. An average coverage of 52x was established and in total 17,848 coding variants were detected. After filtering for 
nonsynonymous variants and variants leading to premature stop codons, which had an allele frequency of $<0.01$ or were absent in SNP databases but present in the homozygous candidate regions, only SLC19A3 contained two mutated alleles. The patient was homozygous for a substitution in SLC19A3 (c.20C>A) which resulted in a premature stop codon (p.Ser7Ter). The presence of the mutation was confirmed by Sanger sequencing (Figure $\mathbf{3}$ ).

A C C T G C

Index

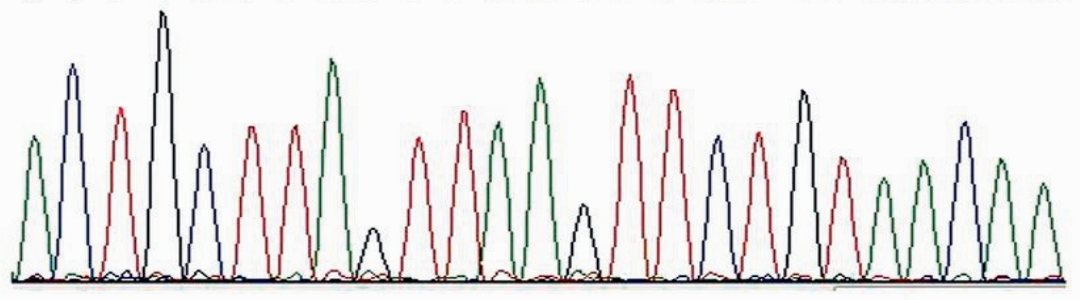

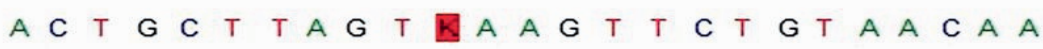

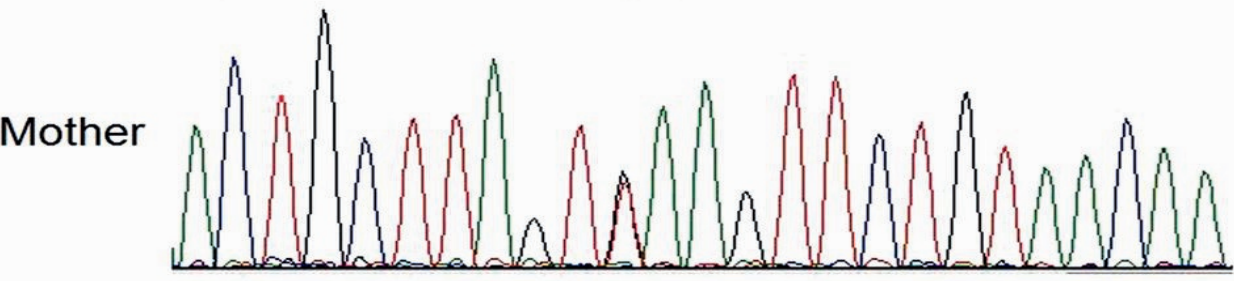

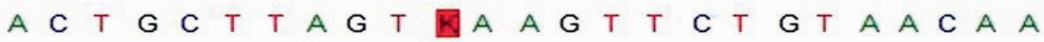

Father

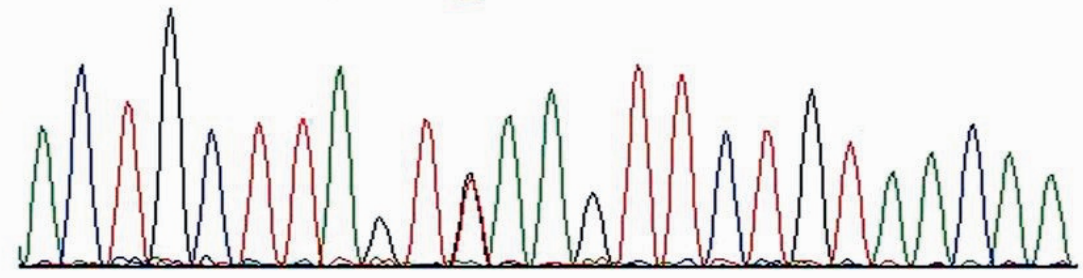

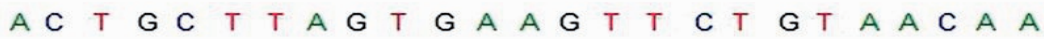

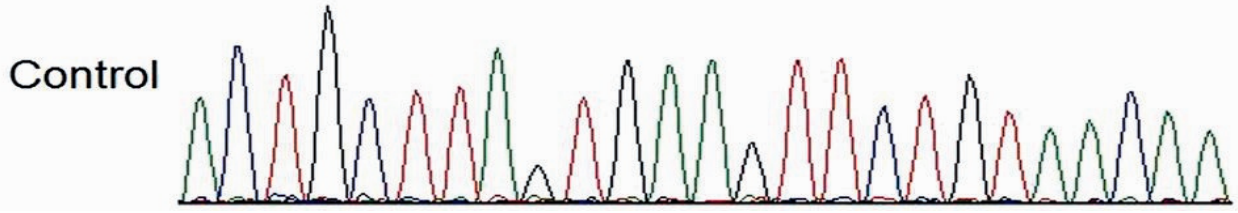

Figure 3. Mutation spectrum family a. All are reverse sequences. 
The patients' affected brother was homozygous for the mutation, whereas both parents and the healthy siblings I:2, II:3 and III:4 were heterozygous. The mutation was not present in 460 Moroccan control alleles. Measurement of the pyruvate dehydrogenase activity in muscle of patient II:5 showed no activity in the absence of thiamine pyrophosphate (TPP) as co-factor whereas the activity was normal in the presence of TPP supporting a thiamine deficiency (Table $\mathbf{1}$ ). Sequence analysis of SLC19A3 in 17 additional patients with Leigh syndrome revealed the same homozygous c.20C>A mutation in two patients of Moroccan origin, from different families (Figure 1; families $b$ and $c$ ). One patient (family $b$ ) showed the same MRI abnormalities as the patients of the first family and was born from consanguineous parents.

Table 1. Pyruvate dehydrogenase activity

\begin{tabular}{|l|l|l|}
\hline & PDH complex activity -TPP & PDH complex activity +TPP \\
\hline Control & $2.8 \pm 0.4$ & $7.2 \pm 2.2$ \\
\hline Patient II:5, Family A & 0 & 8.2 \\
\hline Pyruvate dehydrogenase activity in muscle normalized to CS \\
PDH = Pyruvate dehydrogenase; TPP = thiamine pyrophosphate \\
\hline
\end{tabular}

The parents of the other patient (family c) were not related. This family had previously been tested for linkage using the Human Mapping 10K GeneChips for genotyping and Merlin for linkage analysis. Although not statistically significant, genomic regions on several chromosomes segregated with the disease (maximum LODscore 1.58) including a region from 222 to $231 \mathrm{Mb}$ on chromosome 2 in which SLC19A3 is located. Segregation analysis of the mutation in this family showed a perfect genotype-phenotype association. Unfortunately no material was available for segregation analysis in family $b$.

As all three families carried the same mutation and originated from Morocco, markers surrounding the mutation were genotyped to test for a common haplotype. The markers D2S2354, D2S159, D2S401, D2S439, D2S362 and D2S2297, located within $0.3-1.8 \mathrm{Mb}$ of SLC19A3, were used to determine the haplotype of this region (Figure 1). A shared genotype was detected for all patients for markers D2S159 and D2S401. Possible recombination events in the 
regions between markers D2S2354 and D2S159 and markers D2S401 and D2S439 resulted in a 0.74-1.35 Mb shared region between all affected individuals which includes SLC19A3. Additionally, 230 Moroccan controls were genotyped for these markers to determine the allele frequencies in the Moroccan population. These frequencies were used for the calculation of the linkage disequilibrium (LD) between the SLC19A3 mutation and the recombinant markers D2S22354 and D2S439 which were $\delta=0.542125$ and $\delta=0.664317$ respectively. Taken into account the genetic distances between the mutation and these two markers, the c.20C>A mutation in SLC19A3 was estimated to have occurred between 50 and 70 generations ago. Assuming 25 years per generation, this would mean that the mutation arose about 1250 to 1750 years ago.

To confirm a causal role for this mutation in the biochemical and clinical phenotype, we performed thiamine uptake assays in HEK293 cells overexpressing either wild-type or mutant SLC19A3 (Figure 4). Low and high concentration of radiolabeled thiamine was used, testing both high-affinity, low capacity uptake and low-affinity, high capacity uptake. A clear increase (approx. 4-fold) in thiamine uptake was observed in cells transfected with wild-type SLC19A3, confirming its function as functional thiamine transporter. In contrast, cells expressing mutant SLC19A3 do not show enhanced thiamine uptake when compared to mock-transfected cells, demonstrating that the mutation results in an absent or non-functional protein.

Low and high concentration of radiolabeled thiamine was used, testing both highaffinity, low capacity uptake and low-affinity, high capacity uptake. A clear increase (approx. 4-fold) in thiamine uptake was observed in cells transfected with wild-type SLC19A3, confirming its function as functional thiamine transporter. In contrast, cells expressing mutant SLC19A3 do not show enhanced thiamine uptake when compared to mock-transfected cells, demonstrating that the mutation results in an absent or non-functional protein. 

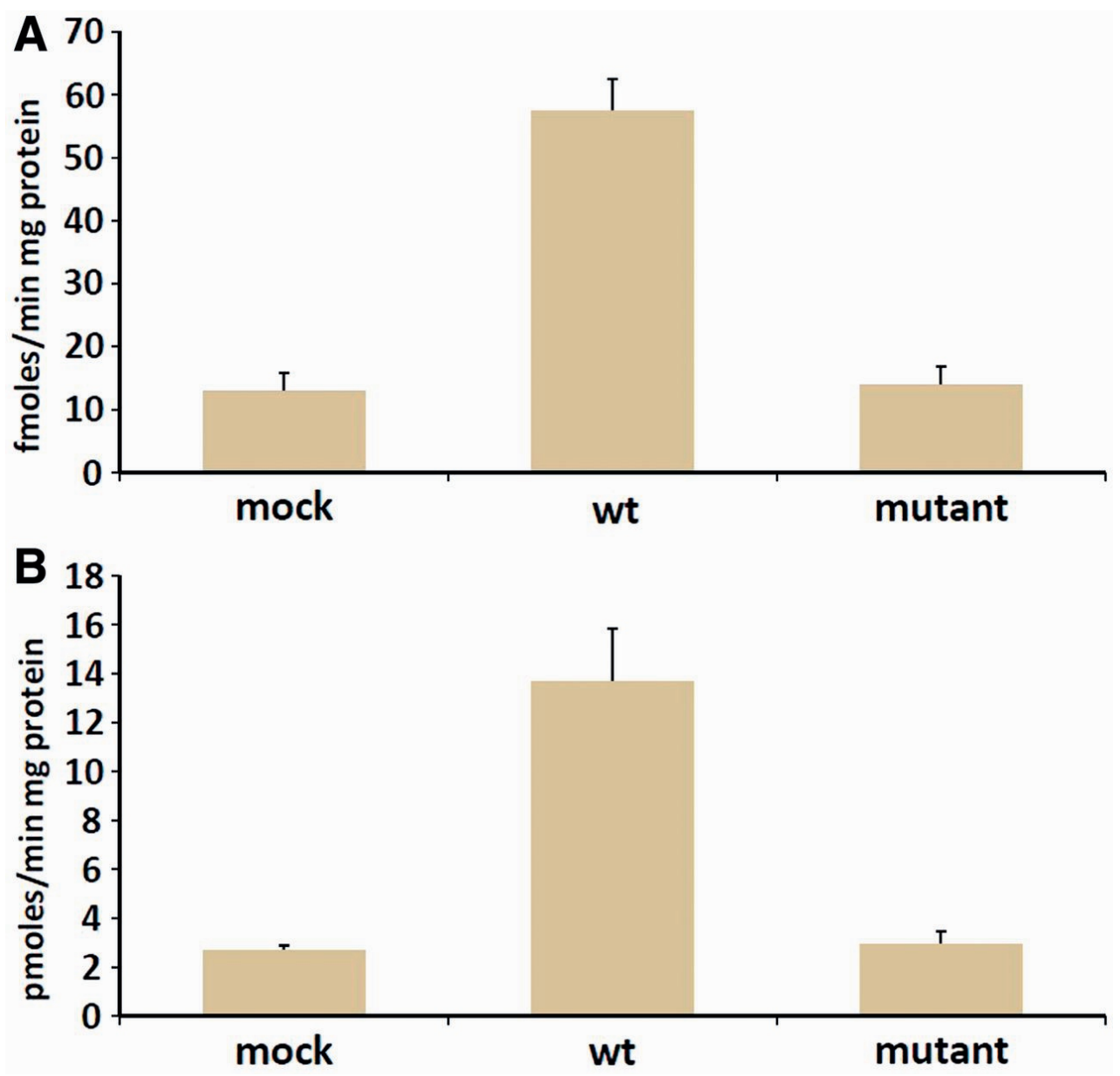

Figure 4. Thiamine transport assay. Thiamine uptake in HEK293 cells overexpressing empty vector (mock), wild-type (wt) or mutant (mutant) SLC19A3 in the presence of (a) a low concentration of radiolabeled thiamine $(15 \mathrm{nM})$ or $(b)$ a high concentration of radiolabeled thiamine $(10 \mu \mathrm{M})$. Mock and mutant transfected cells take up only a marginal amount of thiamine while wild-type SLC19A3 overexpressing cells take up 4 times more thiamine. Error bars indicate the standard deviation. 


\section{Discussion}

By combining whole exome sequencing with homozygosity mapping we identified a homozygous nonsense mutation in SLC19A3 (c.20C>A; p.Ser7Ter) causing Leigh syndrome in a Moroccan consanguineous family. Because candidate regions after homozygosity mapping contained far too many genes for systematic sequence analysis and no obvious candidate gene, whole exome sequencing was performed yielding an average coverage of $52 x$ and 17848 coding variants. Variants were filtered in various steps to exclude those unlikely to be pathogenic. In the first step, nonsynonymous variants and variants leading to premature stop codons were selected as these are most likely to affect protein function. In the next step, variants which are present in different SNP databases like dbSNP132 and 1000 genomes [11][55] and have an allele frequency $>0.01$ were excluded. Subsequently only genes with homozygous variants were selected based on the autosomal recessive inheritance and consanguinity in the family. Finally, only the variants located within the homozygous candidate regions were considered candidates. This strategy revealed only one strong candidate, a homozygous nonsense mutation in SLC19A3, a gene previously reported to be involved in neurological disorders. The sequence of SLC19A3 in 17 additional Leigh patients was analysed and lead to the detection of the same homozygous C.20C>A in two unrelated patients of Moroccan origin. The brain MRI from patient II:5 of family $b$ and patient II:9 of family $c$ showed the same pattern of involvement of putamen, thalamus and cerebellar region as patient II:5 of family a suggesting the same disease. Marker analysis of the region surrounding the mutation in the three families showed a 0.74-1.35 $\mathrm{Mb}$ shared region between all affected individuals. This combined with the fact that the three families originated from the same province Al Hoceima in Northern Morocco supports the presence of a founder effect. Calculations to assess the age of the mutation revealed that the mutation occurred $1250-1750$ years ago.

Metabolic investigations in all families indicated a mitochondrial defect due to possible deficiency of the respiratory chain, the pyruvate dehydrogenase, the 2 ketoglutaric acid dehydrogenase or the branched chain ketoacid dehydrogenase. A defect in the respiratory chain was excluded by the biochemical enzyme measurements. The normal oxygen consumption with glutamate and defective 
oxygen consumption with pyruvate indicated a defect in pyruvate dehydrogenase. However, the pyruvate dehydrogenase activity measured in the presence of thiamine pyrophosphate was found to be either normal or increased, indicating there is no genetic defect of the pyruvate dehydrogenase complex but a defect of a cofactor. The cofactor which could explain the increase in the different metabolites such as lactate, pyruvate, 2-ketoglutaric acid, leucin and isoleucin is thiamine pyrophosphate, the active form of thiamine, indicating a possible defect in thiamine, thiamine transport or in the synthesis of thiamine pyrophosphate.

SLC19A3 is one of the two thiamine transporters present in the plasma membrane. In our patients, no functional SLC19A3 is predicted to be present, due to the premature stop codon, preventing the transport of thiamine in the cytoplasm by SLC19A3. Mutations in SLC19A3 have previously been associated with neurological disorders like, biotin responsive basal ganglia disease (BBGD) $[12,13]$, Wernicke's-like encephalopathy [14] and epileptic spasm in early infancy, severe psychomotor retardation and progressive atrophy [15]. Our results identified Leigh syndrome with specific MRI abnormalities as a new neurological disease phenotype associated with mutations in SLC19A3. It has been suggested that loss-of-function mutations are associated with the BBGD phenotype [15]. However, our patients had Leigh syndrome caused by a homozygous nonsense mutation which does not support this hypothesis. Moreover, the c.980-14A>G mutation described by Debs et al in BBGD patients does probably not result in a complete skipping of exon 4 as wild-type transcript was still detectable in patients which indicates there is a decreased amount of protein rather than a complete absence [13]. Additionally, the BBGD families show typically striatal necrosis with caudate neurodegeneration which was not observed in any of our families, not even in the severely affected patients. Mutations in SLC19A2, the other thiamine transporter, have been described to cause megaloblastic anemia [16-18]. The affected tissues in SLC19A2 or SLC19A3 deficiency correlate with the difference in expression of both thiamine transporters.

Thiamine is imported into the cytosol by either SLC19A2 or SLC19A3 and converted into the active form, thiamine pyrophosphate (TPP), by thiamine pyrophosphokinase 1 (TPK1). TPP is an important cofactor in the cytosol for 
transketolase and in the mitochondria for pyruvate dehydrogenase (PDH), 2ketoglutarate dehydrogenase $(2-\mathrm{KGDH})$ and branched chain a-keto acid dehydrogenase $(B C K D H)$. As SLC19A3 is the low affinity high capacity transporter, this will most likely lead to a deficiency of the cofactor TPP and of the TPP dependent processes. Whereas the other thiamine transporter, SLC19A2, is a high affinity low capacity transporter with a different tissue expression pattern. It is highly unlikely SLC19A2 can compensate the absence of a functional SLC19A3. Thiamine, or vitamin $B_{1}$, is not synthesized by humans or other animals and is required in the diet as the thiamine reserves only last for 20 days. Absence of thiamine in the diet or due to alcohol consumption leads to Beri-Beri or WernickeKorsakoff syndrome. Overlapping symptoms with Leigh syndrome are hypertonia, hypotonia, muscle weakness, dystonia, apneu, epilepsy, feeding problems, nystagmus and deafness.

A central role for thiamine deficiencies or thiamine transport defects in Leigh syndrome and related neurological disease can be further deduced from the clinical phenotypes associated with mutations in TPK1, the mitochondrial thiamine transporter (SLC25A19) and the TPP dependent processes. Whereas patients with mutations in TPK1 presented with variable degrees of ataxia, psychomotor retardation, progressive dystonia and lactic acidosis [19], mutations in SLC25A19 have been described in patients with bilateral striatal necrosis and chronic progressive polyneuropathy [20]. Mutations in PDHA1 and $P D H B$, both encoding subunits of the $E_{1}$ component of the PDH and sharing a TPP binding site, have been reported to cause Leigh syndrome [21, 22]. Finally, mutations have been identified in different subunits of the BCKDH in patients with maple syrup urine disease [23]. Remarkably, all mutations in proteins involved in the thiamine metabolism, with the exception of SLC19A2, result in severe neurological symptoms demonstrating the importance of thiamine in brain development.

Previous studies have shown that SLC19A3 expression is dependent on biotin levels. The phenotype in BBGD patients is likely the result of insufficient thiamine transport which can partly be restored by overexpression of SLC19A3 via high doses of biotin. This supports our hypothesis that mutations in BBGD patients lead to a decreased amount or activity of SLC19A3 instead of a completely nonfunctional protein. Besides biotin, also administration of high doses of thiamine 
has been reported to improve the clinical phenotype [14]. In this study it is suggested that the efficiency of high doses of thiamine indicates that dysfunction of SLC19A3 may induce expression of SLC19A2 increasing intracellular thiamine transport. The only patients in our families who survived childhood (patients II:3 and II:9 of family $c$ ) had been treated with a cocktail of vitamins, including thiamine and biotin. As it is highly unlikely that overexpression of the mutated SLC19A3 gene will be beneficial, this treatment might have induced a partially compensating up-regulation of SLC19A2, which could be sufficient for patients carrying this nonsense mutation to survive childhood. For patients with TPK mutations, TPP has been suggested as a therapeutic option [19]. This might also be an option for patients with SLC19A3 mutations. However, as stated by Mayr et al., the effectivity and safety of TPP as a drug still needs to be demonstrated and it is unclear whether TPP can cross the blood brain-barrier [19]. In summary, we identified a nonsense mutation in SLC19A3 in a consanguineous Moroccan family with Leigh syndrome, expanding the spectrum of neurological disorders associated with mutations in SLC19A3 and pathways affected in Leigh syndrome. Our results further indicate that mutational screening of SLC19A3 should be considered in patients with mitochondrial disorders and neurological symptoms, especially in specific parts of Morocco. Finally, these patients might benefit from high doses of biotin or, more likely, thiamine which would make this a partly treatable form of Leigh syndrome.

\section{Acknowledgements}

We would like to warmly acknowledge the late neurologist Prof. dr. Herman F.M. Busch (Aug 21, 1929-Jan 21, 2005; Erasmus MC Rotterdam, The Netherlands), who has been involved in the clinical investigations, and L.M. Hussaarts for excellent technical support.

\section{Funding}

The work is supported by the Alma in Silico project, which is financed by the Interreg IV European funds, The Walloon Region, The North Rhine Westphalia, The Flemish Community, The Belgian Province of Limburg and The Dutch Province of Limburg, as well as by the Universities of Maastricht and Liège, by the Prinses Beatrix Fonds (grant W.OR11-24) and the Stichting Metakids. 


\section{References}

1. Tucker, E.J., A.G. Compton, and D.R. Thorburn, Recent advances in the genetics of mitochondrial encephalopathies. Curr Neurol Neurosci Rep, 2010. 10(4): p. 277-85.

2. Matthews, P.M., et al., Molecular genetic characterization of an X-linked form of Leigh's syndrome. Ann Neurol, 1993. 33(6): p. 652-5.

3. Grafakou, O., et al., Leigh syndrome due to compound heterozygosity of dihydrolipoamide dehydrogenase gene mutations. Description of the first E3 splice site mutation. Eur J Pediatr, 2003. 162(10): p. 714-8.

4. Mootha, V.K., et al., Identification of a gene causing human cytochrome $c$ oxidase deficiency by integrative genomics. Proc Natl Acad Sci U S A, 2003. 100(2): p. 605-10.

5. Pequignot, M.O., et al., Mutations in the SURF1 gene associated with Leigh syndrome and cytochrome C oxidase deficiency. Hum Mutat, 2001. 17(5): p. 374-81.

6. Metzker, M.L., Sequencing technologies - the next generation. Nat Rev Genet, 2010. 11(1): p. 31-46.

7. Ku, C.S., N. Naidoo, and Y. Pawitan, Revisiting Mendelian disorders through exome sequencing. Hum Genet, 2011. 129(4): p. 351-70.

8. Scholte, H.R., et al., Oxidative phosphorylation in human muscle in patients with ocular myopathy and after general anaesthesia. Biochim Biophys Acta, 1990. 1018(2-3): p. 211-6.

9. Seelow, D., et al., HomozygosityMapper--an interactive approach to homozygosity mapping. Nucleic Acids Res, 2009. 37(Web Server issue): p. W593-9.

10. Risch, N., et al., Genetic analysis of idiopathic torsion dystonia in Ashkenazi Jews and their recent descent from a small founder population. Nat Genet, 1995. 9(2): p. 152-9.

11. A map of human genome variation from population-scale sequencing. Nature, 2010. 467(7319): p. 1061-73.

12. Zeng, W.Q., et al., Biotin-responsive basal ganglia disease maps to $2 q 36.3$ and is due to mutations in SLC19A3. Am J Hum Genet, 2005. 77(1): p. 16-26.

13. Debs, R., et al., Biotin-responsive basal ganglia disease in ethnic Europeans with novel SLC19A3 mutations. Arch Neurol, 2010. 67(1): p. 126-30.

14. Kono, S., et al., Mutations in a thiamine-transporter gene and Wernicke'slike encephalopathy. N Engl J Med, 2009. 360(17): p. 1792-4.

15. Yamada, K., et al., $A$ wide spectrum of clinical and brain MRI findings in patients with SLC19A3 mutations. BMC Med Genet, 2010. 11: p. 171.

16. Labay, V., et al., Mutations in SLC19A2 cause thiamine-responsive megaloblastic anaemia associated with diabetes mellitus and deafness. Nat Genet, 1999. 22(3): p. 300-4. 
17. Raz, T., et al., The spectrum of mutations, including four novel ones, in the thiamine-responsive megaloblastic anemia gene SLC19A2 of eight families. Hum Mutat, 2000. 16(1): p. 37-42.

18. Scharfe, C., et al., $A$ novel mutation in the thiamine responsive megaloblastic anaemia gene SLC19A2 in a patient with deficiency of respiratory chain complex I. J Med Genet, 2000. 37(9): p. 669-73.

19. Mayr, J.A., et al., Thiamine pyrophosphokinase deficiency in encephalopathic children with defects in the pyruvate oxidation pathway. Am J Hum Genet, 2011. 89(6): p. 806-12.

20. Spiegel, R., et al., SLC25A19 mutation as a cause of neuropathy and bilateral striatal necrosis. Ann Neurol, 2009. 66(3): p. 419-24.

21. Schiff, M., et al., Leigh's disease due to a new mutation in the PDHX gene. Ann Neurol, 2006. 59(4): p. 709-14.

22. Quintana, E., et al., PDH E(1)beta deficiency with novel mutations in two patients with Leigh syndrome. J Inherit Metab Dis, 2009.

23. Nobukuni, Y., et al., Heterogeneity of mutations in maple syrup urine disease (MSUD): screening and identification of affected E1 alpha and E1 beta subunits of the branched-chain alpha-keto-acid dehydrogenase multienzyme complex. Biochim Biophys Acta, 1993. 1225(1): p. 64-70. 


\section{Legends of supplemental figures}

Supplementary Table 1. PCR primers for SLC19A3

\begin{tabular}{|c|c|c|c|}
\hline & PCR primers $5^{\prime} \rightarrow 3^{\prime}$ & & \\
\hline $\begin{array}{l}\text { Gene- } \\
\text { Exon }\end{array}$ & Forward & Reverse & $\begin{array}{l}\text { Produ } \\
\text { ct } \\
\text { size } \\
\text { (bp)* }\end{array}$ \\
\hline $\begin{array}{l}\text { SLC19A } \\
3-E \times 2\end{array}$ & CAACAGAGCGACCCTCC & AATCAGGCTCACTGAGTTTAAT & 386 \\
\hline $\begin{array}{l}\text { SLC19A } \\
\text { 3-Ex3A }\end{array}$ & ATGTTTCTCCGTAATGCGAATAA & ACCGACCCTGCTGTGTA & 432 \\
\hline $\begin{array}{l}\text { SLC19A } \\
\text { 3-Ex3B }\end{array}$ & GGCCTACTACGCCTACATATACA & AACGTCCACAGTCACATTG & 421 \\
\hline $\begin{array}{l}\text { SLC19A } \\
\text { 3-Ex3C }\end{array}$ & GAAGAGCAGAAACCCACATC & CCGTGACTTTCATTCTTGAC & 434 \\
\hline $\begin{array}{l}\text { SLC19A } \\
3-E x 4\end{array}$ & $\begin{array}{l}\text { ATTTATCTTTCAGAATTACCCGA } \\
\text { G }\end{array}$ & $\begin{array}{l}\text { GTTGGCTTAGTTGTGTCTAATT } \\
\mathrm{T}\end{array}$ & 448 \\
\hline $\begin{array}{l}\text { SLC19A } \\
3-E \times 5\end{array}$ & GCTGGAGGTGGGTGACA & $\begin{array}{l}\text { TITAATTGCTTAACCAAGGAA } \\
\text { CTC }\end{array}$ & 354 \\
\hline $\begin{array}{l}\text { SLC19A } \\
\text { 3-Ex6 }\end{array}$ & TCAGTTACCCCATAATCCAAGC & CAAAGCATGTCAAGTTATGGCA & 381 \\
\hline $\begin{array}{l}\text { * Exten } \\
\text { Rev:CA }\end{array}$ & $\begin{array}{l}\text { with M13-sequence (For:TGTA } \\
\text { AACAGCTATGACC) }\end{array}$ & CGACGGCCAGT and & \\
\hline
\end{tabular}


CHAPTER 5

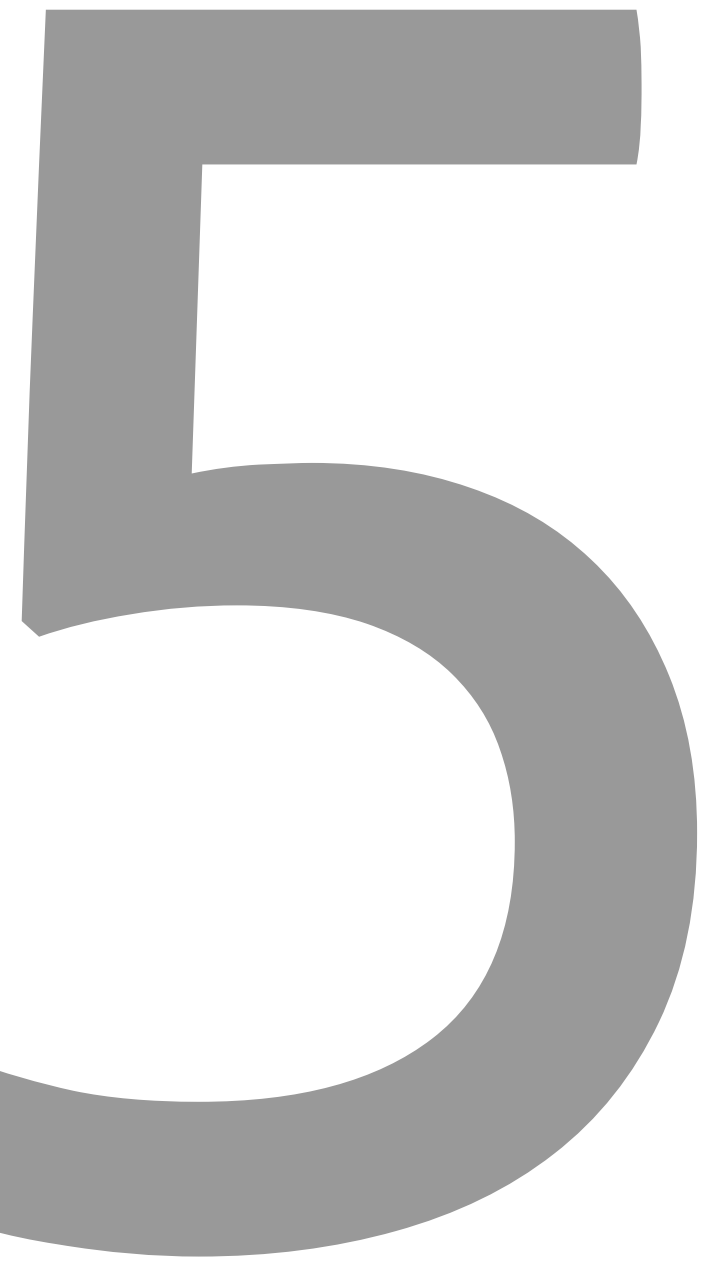




\section{Genetic Defects in mtDNA-encoded Protein Translation Cause Paediatric, Mitochondrial Cardiomyopathy with Early-onset Brain Disease}

Rick Kamps, Radek Szklarczyk, Tom E. Theunissen, Debby M.E.I. Hellebrekers, Suzanne C.E.H. Sallevelt, Iris B. Boesten, Bart de Koning, Bianca J. van den Bosch, Gajja S. Salomons, Marisa Simas-Mendes, Rob Verdijk, Kees Schoonderwoerd, Irenaeus F.M. de Coo, Jo M. Vanoevelen*, Hubert J.M. Smeets*

*Joint senior author, equal contribution 


\section{Abstract}

This study aims to identify gene defects in paediatric cardiomyopathy and earlyonset brain disease with oxidative phosphorylation (OXPHOS) deficiencies. We applied Whole-Exome Sequencing in 3 patients with paediatric cardiomyopathy and early-onset brain disease with OXPHOS deficiencies. The brain pathology was studied by MRI analysis. In consanguineous patient 1 we identified a homozygous intronic variant (c.850-3A $>\mathrm{G}$ ) in the $Q R S L 1$ gene, which was predicted to cause abnormal splicing. The variant segregated with the disease and affected the protein function, which was confirmed by complementation studies, restoring OXPHOS function only with wild-type QRSL1. Patient 2 was compound heterozygous for two novel affected and disease causing variants (c. [253G>A]; [938G>A]) in the MTO1 gene. In patient 3 we detected one unknown affected and disease causing variants (c.2872C $>T$ ) and one known disease causing variant (c.1774C $>T$ ) in the AARS2 gene. The c.1774C $>T$ variant was present in the paternal copy of the AARS2 gene, the c.2872C $>T$ in the maternal copy. All genes were involved in translation of mtDNA-encoded proteins. Defects in mtDNA-encoded protein translation lead to severe paediatric cardiomyopathy and brain disease with OXPHOS abnormalities. This suggests that the heart and brain are particularly sensitive to defects in mitochondrial protein synthesis during late embryonic or early postnatal development, probably due to the massive mitochondrial biogenesis occurring at that stage. If both the heart and brain are involved, the prognosis is poor with a likely fatal outcome at young age. 


\section{Introduction}

In this study we searched for the genetic defect in paediatric patients with severe cardiomyopathy and early-onset brain disease with deficiencies in oxidative phosphorylation (OXPHOS). This is often lethal at a very young age. The affected hearts were mostly thick-walled and diagnosed as hypertrophic cardiomyopathy (HCM). The brain pathology was investigated with MRI. Additionally, muscle, liver, and developmental abnormalities were observed, this is not uncommon for OXPHOS diseases as the OXPHOS systems consists of 5 enzyme complexes, which generate ATP, the prime source of cellular energy, and OXPHOS defects often affect multiple organs and more particularly those organs with a high energy requirement. Overall, OXPHOS diseases have a prevalence of about 1 in 85000 . Genetically, 13 of the structural proteins of the OXPHOS complex are encoded by the mtDNA, which has a genetic code different from the nuclear DNA and therefore also contains the necessary tRNA and rRNA genes to generate mtDNA encoded proteins [1]. The vast majority of the OXPHOS proteins, but also the proteins involved in replication, transcription and translation of the mtDNA, in assembly of the OXPHOS complexes, and in mitochondrial quality control are encoded by hundreds of nuclear genes. This implies that these disorders can have a dual genetic origin [2]. The extreme variability of the clinical manifestations and the high number of genes potentially precludes genotype-phenotype correlations and complete, unbiased approaches, like whole exome sequencing, have become the preferred and successful strategy to reveal the underlying genetic cause.

We performed Whole-Exome Sequencing (WES) in 3 patients from unrelated families, one consanguineous and two non-consanguineous, in which mtDNA defects were excluded as cause. We filtered the consanguineous family for homozygous variants affecting protein function in regions of homozygosity, whereas for the non-consanguineous families first a targeted analysis of 412 mitochondrial disease genes and functionally or structurally related genes was performed [3]. When negative, this was followed by an analysis of all genes captured and sequenced. For both approaches an autosomal recessive disease model was used [4-8]. In each family a severe defect in a different gene was identified causing the disease, but all 3 genes were involved in translation of mtDNA-encoded proteins. 


\section{Material and Methods}

\section{Patients with Paediatric Cardiomyopathy, Early-Onset Brain Disease and OXPHOS Deficiency}

\section{Patient I (Family I)}

Patient 2.2, the female proband of a consanguineous Turkish couple, was prenatally diagnosed with hypertrophic cardiomyopathy using fetal echocardiography (Ultrasound) and postnatally confirmed by electrocardiography (ECG). From birth she developed encephalopathy, failure to thrive, and lactic acidosis. Magnetic resonance imaging (MRI) of the brain at 5 months showed diffuse cerebral atrophy with T2 weighed imaging including hyper-intensive abnormalities in globus pallidus and putamen and also infratentorial in the dorsal brain stem, medulla oblongata and mesencephalon, which is characteristic for Leigh syndrome (Figure 1, the female patient 2.2).

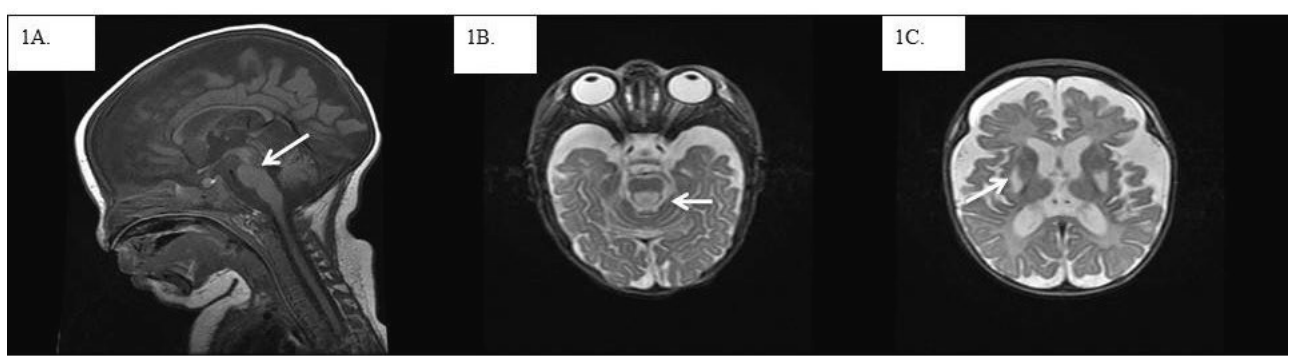

Figure 1. MRI of the brain data from patient I.2.2 1A.T1 weighted sagittal image: small corpus callosum, atrophy of the cerebellar vermis, abnormal intensity dorsal pons and mesencephalic region (arrow). 1B. Axial T2 weighted images showed subdural effusions, diffuse atrophy, frontal>occipital and hyperintensities in dorsal mesencephalon (arrow) in figure. 1C. Hyperintensities in the dorsolateral part of the putamen (arrow). All three figures showed Leigh syndrome.

MRI spectroscopy of the white matter showed high lactic acid peak, and elevated biochemistry data for choline/creatine ratio, consistent with demyelination. She died at the age of 6 months. Her brother (2.1), with a comparable phenotype, died in utero and both were prenatally diagnosed with hypertrophic cardiomyopathy using fetal echocardiography (Ultrasound). Patient 2.2 was also 
prenatally diagnosed with a growth delay and a head lag. This head lag in infants is showing a developmental delay in head and neck muscle control. In conclusion the pregnancy of 2.1 and 2.2 was not a normal development in contrast to 2.3 and 2.4. Their parents, a second sister (2.3), and the youngest brother (2.4) were healthy (Figure 2A).

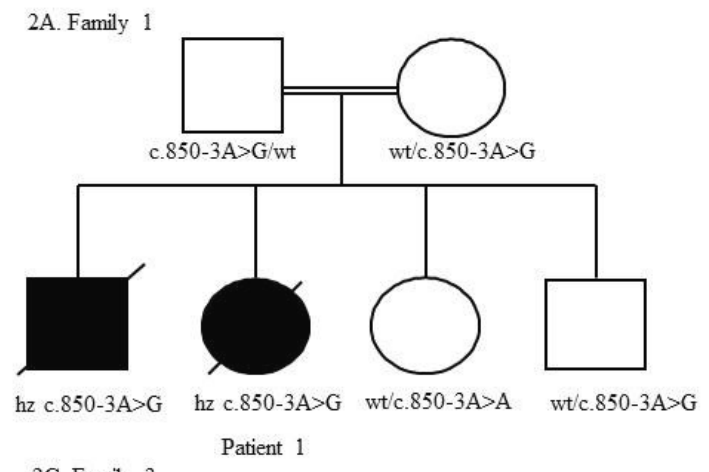

2B. Family 2
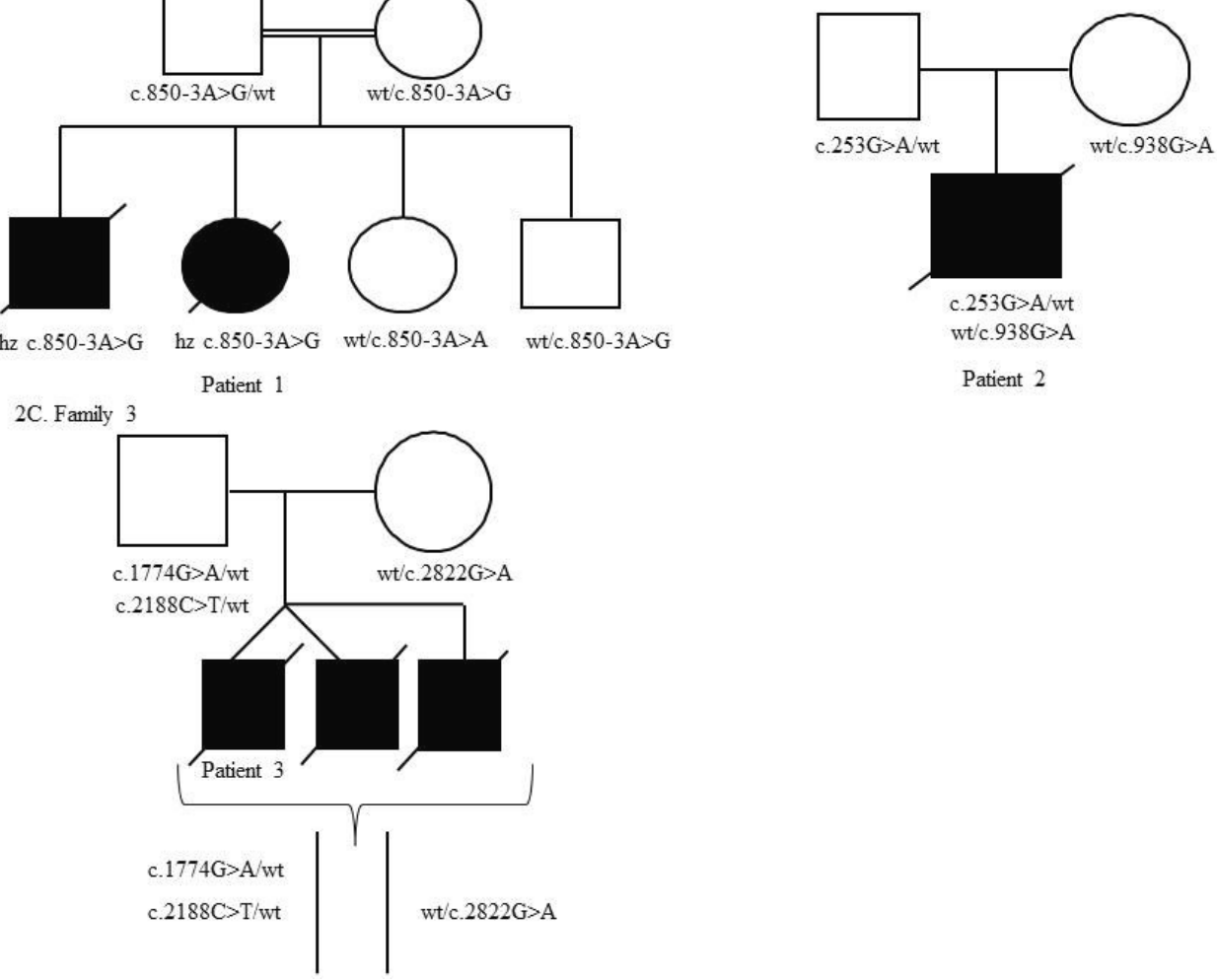

Figure 2. Pedigree of 3 families with paediatric cardiomyopathy and Leigh (Family A) or Leigh-like syndrome (Family B and C) with multiple OXPHOS deficiencies. 2A. Patient I.2.1 and I.2.2 from a consanguineous marriage carried a homozygous splicing defect in QRSL1 gene (NC_000006.11(NM_018292.4)). 2B. Patient II.2.1 showed two novel variants in the MTO1 gene (NM_001123226.1). 2C. Patient III.2.1, III.2.2, and III.2.3 showed two variants in the AARS2 gene (NM_020745.3).

The relative activities of OXPHOS enzyme complexes in muscle tissue biopsy of patient 2.2 were normalized to Citrate Synthase (CS); CI=41\%, CII $=68 \%$, $\mathrm{CIII}=70 \%, \mathrm{CIV}=71 \%, \mathrm{CV}=40 \%$. Skin fibroblast $(2.2)$ OXPHOS data showed 
$\mathrm{CI}=43 \%, \mathrm{CII}=81 \%, \mathrm{CIII}=86 \%, \mathrm{CIV}=54 \%, \mathrm{CV}=64 \%$. This indicates a complex $\mathrm{I}$ and $\mathrm{V}$ deficiency in muscle and a complex I, IV and V deficiency in fibroblasts.

Patient 2 (Family II)

Patient 2.1 was diagnosed with a severe paediatric, hypertrophic cardiomyopathy (HCM) by ECG and died at the age of 1.5 year. He was the only child of nonconsanguineous Dutch healthy parents (Figure 2B) during a normal pregnancy. He showed diffuse cerebral atrophy with intraventricular septa on the MRI, characteristic for brain disease, and high levels of alanine and lactic acid, suggesting a mitochondrial disorder. The relative activities of OXPHOS enzyme complexes in muscle biopsy material of patient 2.1 were normalized to $\mathrm{CS}$; $\mathrm{CI}=11 \%, \quad \mathrm{CII}=128 \%, \mathrm{CIII}=25 \%, \quad \mathrm{CIV}=11 \%, \quad \mathrm{CV}=61 \%$, showing a severe deficiency of complex I, III and IV. Skin fibroblast data showed a decreased activity of $\mathrm{CI}=50 \%$, unfortunately CII, CIII, CIV, CV were not determined.

\section{Patient 3 (Family III)}

Patient 2.1 was born dysmature and was diagnosed at birth with severe hypertrophic cardiomyopathy (HCM) by ECG, and encephalomyopathy, persistent hypotonia, lung hypoplasia, and also high levels of lactic acid. Two other male siblings showed a similar phenotype and all three affected siblings died within 2 weeks after birth (2.2 within 1 day, 2.1 within 4 days, and 2.3 within 16 days) (Figure 2C). The Dutch healthy parents were unrelated. Patient 2.1 and 2.2 patients were described as a spontaneous twin pregnancy and no complications before planned section Caesarea. Nevertheless, after birth patient 2.2 showed immediately lung hypoplasia and died after 2 hours post-mortem. No further information of patient 2.3 was available. Muscle biopsy (2.1) taken directly after birth showed many COX negative fibers with no other abnormalities. Postmortem brain pathology (2.1) confirmed the postmortem MRI and showed degeneration of the fasciculus gracilis and cuneatus with some vacuolization of the substantia nigra, characteristic for Leigh-like syndrome. The HCM was also confirmed during the postmortem heart pathology (2.1). The heart tissue was observed by transmission electron microscopy (TEM) and showed abnormal mitochondria, which were less in amount and large in size (Data not shown). The relative 
activities of OXPHOS enzyme complexes in muscle tissue biopsy of patient 2.1 were normalized to $\mathrm{CS}$; $\mathrm{CI}=27 \%, \mathrm{CII}=120 \%, \mathrm{CIII}=11 \%, \mathrm{CIV}=6 \%, \mathrm{CV}=48 \%$. Skin fibroblast data showed activities of $\mathrm{CI}=45 \%, \mathrm{CII}=67 \%, \mathrm{CIII}=119 \%, \mathrm{CIV}=97 \%$, $\mathrm{CV}=107 \%$. In muscle all complexes showed a decreased activity, except complex II, whereas in fibroblasts only complex I was decreased.

\section{Homozygosity Mapping}

Homozygosity mapping was performed using the Affymetrix GeneChip Human Mapping 250K SNP array (Affymetrix, Santa Clara, US) using peripheral blood DNA of consanguineous parents and siblings (Family I). The DNA was labelled and processed according to the manufacturer's instructions. Genotypes were generated by the Affymetrix Genotyping Analysis Software (GTYPE). Homozygous regions were determined by the online tool Homozygosity Mapper,(http://www.homozygositymapper.org/).

\section{Whole-Exome Sequencing and Analysis}

Genomic DNA was isolated from peripheral blood leukocytes using the DNeasy Blood \& Tissue kit (Qiagen, Hilden, Germany). The quality of the genomic DNA was determined on a NanoDrop (Wilmington, USA) and by Agarose gel electrophoresis and finally quantified with PicoGreen on a Qubit fluorometer (Invitrogen, Bleiswijk, The Netherlands). A total of $4 \mu \mathrm{g}$ starting material was used, which was further purified with a DNA Clean \& Concentrator TM (ZymoResearch, BaseClear Diagnostics, Leiden, The Netherlands). The genomic DNA was fragmented to $150-200$ bp lengths by shearing on a Covaris S2 (Covaris Inc, Massachusetts, US). The exome was captured with the SureSelect Human All Exon V4 plus UTR's kit (Agilent, Technologies, Amstelveen, The Netherlands) for PairedEnd Illumina Sequencing platforms (Illumina, Eindhoven, The Netherlands) using the standard SureSelect protocol (version 1.3). Quality was controlled on a Bioanalyzer 2100 (Agilent). Basecalling was performed using Casava 1.8 (Illumina). Reads were aligned to the human reference genome (UCSC hg19) using Burrows-Wheeler-Alignment tool (BWA) version 0.5.9. Single base variants were identified using Sequence-Alignment-MAP tools (SAM) version 0.1.16 and annotated according an in-house annotation Python/R script that matched 
variants to the RefGene, GenCode v19, and dbSNP144 hg19 tracks from the UCSC genome browser. Variants between the proband and the reference sequence were annotated and listed in an Excel file for further analyses. Two filtering strategies were applied. In the consanguineous patient I.2.2 homozygous variants from homozygosity regions were analysed. In the non-consanguineous patients II.2.1 and III.2.1, first a diagnostic panel of 412 mitochondrial genes was analysed for compound heterozygous variants, and if negative all captured genes would be analysed.

All variants were filtered for a predicted functional effect (nonsense, missense, INDELs and splice site variants) and frequency ( $<1 \%$ or absent in sequence databases (dbSNP144, Exome Variant Server (EVS), Exome Sequencing Project (ESP), Exome Aggregation Consortium (ExAC) and in-house patient database). Possible splice site variants were analysed with Maximum Entropy Modeling of Short Sequence Motifs (MaxEnt), Neural Networks Berkeley Drosophila Genome Project (NNSPLICE) and Human Splicing Finder (HSF). Missense variants were classified, using SIFT (http://sift.jcvi.org/) and PolyPhen-2 (http://genetics.bwh.harvard.edu/pph2/). Additionally, in the consanguineous patient I.2.2, novel genes carrying variants were prioritized for their likelihood of being involved in mitochondrial disease using Weighted Gene Expression Tool and database (WeGET: http://weget.cmbi.umcn.nl). Shortly, remaining variants were analysed for their co-expression with genes encoding mitochondrial proteins (mitochondrial system) in mammalian tissues. A weighted average of coexpression with mitochondrial system was calculated for all genes containing rare variants affecting protein function. A higher contribution was assigned to datasets that showed congruent expression (concomitant up- and down-regulation) of the mitochondrial system itself. The variants affecting the protein function and could be the cause of the disease in the patient were submitted to the gene variant database. Links were described as:

1) www.lovd.nl/QRSL1, (QRSL1; \#00100428: patient I.2.2), 2) www.lovd.nl/MTO1, (MTO1; \#00100427: patient II.2.1), and 3) www.lovd.nl/AARS2, (AARS2; \#00100426: patient III.2.1). Relevant variants were sequenced with gene specific primers with an M13 tail and the BigDye Terminator v1.1 Cycle Sequencing kit (Applied Biosystems, Bleiswijk, The 
Netherlands) on an ABI 3730 DNA Analyzer. Data were processed using Sequence Scanner v1.0 from Applied Biosystems.

\section{CDNA Analysis and Complementation Assay of QRSL1}

All primers used are listed in Table 1. RNA was isolated from patient and neonatal dermal human fibroblast cells (NDHF control cells) using Total RNA Isolation Kit according to the manufacturer's manual (Roche Nederland B.V., Almere, The Netherlands). Copy DNA (cDNA) synthesis was performed by the qScript cDNA Synthesis Kit (Quanta Biosciences, Gaithersburg, MD, USA) using $1 \mu \mathrm{g}$ of RNA input. Standard PCR steps: 1) $\left.\left.\left.5 \mathrm{~min} 94^{\circ} \mathrm{C}, 2\right) 1 \mathrm{~min} 94^{\circ} \mathrm{C}, 3\right) 1 \mathrm{~min} 58^{\circ} \mathrm{C}, 4\right) 1.5$ $\left.\min 72^{\circ} \mathrm{C}, 5\right) 7 \mathrm{~min} 72^{\circ} \mathrm{C}$, repeat step 2-4 34 cycles and cooling down to $4{ }^{\circ} \mathrm{C}$. QPCR mRNA expression protocol were designed to generate a cDNA fragment across the site of the QRSL1 variant. QRSL1 mRNA expression was measured by the 7900HT Fast Real-Time PCR System (Applied Biosystems, Foster City, CA, USA) and was based on a primer pairs at exon 7 (209bp amplicon), intron 7- exon 8 (169bp amplicon, splice position), exon 8 (202bp amplicon) and exon 9 (162bp amplicon) of the QRSL1 transcript (NM_018292.4). QRSL1 expression was corrected for the TBP housekeeping gene (TATA box-binding protein, NM_003194.4, primer set in exon 5, 89bp amplicon). Sensimix Sybr Hi-Rox (Bioline, Taunton, MA, USA) was used for cDNA amplification under the following conditions: $2 \min 50^{\circ} \mathrm{C}, 2 \mathrm{~min} 95^{\circ} \mathrm{C}$, followed by 40 cycles of $15 \mathrm{~s} 95^{\circ} \mathrm{C}$ (denaturation) and $1 \mathrm{~min} 60^{\circ} \mathrm{C}$ (annealing and extension). Non-specific primer annealing was excluded by analysis of a primer dissociation curve which was obtained by the following PCR steps: $15 \mathrm{~s} 95^{\circ} \mathrm{C}, 15 \mathrm{~s} 60^{\circ} \mathrm{C}, 15 \mathrm{~s} 60^{\circ} \mathrm{C}$, cooling down to $4^{\circ} \mathrm{C}$. For patient 1 QRSL1 mRNA expression was calculated using the standard $2-\triangle \triangle C t$ method towards a TBP housekeeping gene, and compared to healthy control NDHF cells. The mutant spliced cDNA (NC_000006.11(NM_018292.4):C.850-3A>G) resulted in a percentage wild-type rest level of the normal spliced CDNA in exon 8 . Mutant and wild-type PCR fragments were cloned using the pGEM T Easy cloning system (Promega, Madison, USA). Wild-type QRSL1 was expressed using QRSL1 CDNA (NM_018292.4) pcDNA3.1_DYK clone (Genscript, Piscataway, US). All wild-type and mutant (patient 1 cDNA) QRSL1 constructs were also cloned in a pUltra-Chili Lentiviral 
(Addgene, Cambridge, US) with special restriction enzymes sites primers (with an overhang generating restriction sites for BamHI (GGATCC) and XbaI (TCTAGA).

Table 1. Primers

\begin{tabular}{|c|c|c|}
\hline Primers & Forward & Reverse \\
\hline QRSL1_M13_F & $\begin{array}{l}\text { TGTAAAACGACGGCC } \\
\text { AGTCCCAACCAGATC } \\
\text { TCCAATCTTT } \\
\end{array}$ & \\
\hline QRSL1_M13_R & & $\begin{array}{l}\text { CAGGAAACAGCTATGACCCCA } \\
\text { TATTCGATGCCACTTCTGA }\end{array}$ \\
\hline QRSL1_exon6_F & $\begin{array}{l}\text { TGTGCCAGGAATCTT } \\
\text { AACCAGA }\end{array}$ & \\
\hline QRSL1_exon9_R & & $\begin{array}{l}\text { ACATGGCTTCAGTGGACACAT } \\
\text { C }\end{array}$ \\
\hline Pultra_QRSL1_F & \begin{tabular}{l|} 
CCTTCTAGAATGGAT \\
TACAAGGATGACGAC
\end{tabular} & \\
\hline Pultra_QRSL1_R & & $\begin{array}{l}\text { GCCGGATCCTTATCATTACTGT } \\
\text { TITAGAGA }\end{array}$ \\
\hline QRSL1_pcDNA3.1_XbaI_F & $\begin{array}{l}\text { CTTTCTAGAATGCTG } \\
\text { GGCCGGAGCCTCCG } \\
\text { AGAA } \\
\end{array}$ & \\
\hline QRSL1_pcDNA3.1_BamHI_R & & $\begin{array}{l}\text { GCCGGATCCTTACTGTITTAG } \\
\text { AGAGAC }\end{array}$ \\
\hline QRSL1 QPCR_Exon 7_F & $\begin{array}{l}\text { TGTGCCAGGAATCTT } \\
\text { AACCAGA }\end{array}$ & \\
\hline QRSL1 QPCR_Exon 7_R & & $\begin{array}{l}\text { ACTGGGAAGCATGAATGGTTT } \\
\text { A }\end{array}$ \\
\hline QRSL1QPCR_Intron 7_Exon 8_F & $\begin{array}{l}\text { TCCAGCCTAGGTGAC } \\
\text { AGAGTGA }\end{array}$ & \\
\hline QRSL1 QPCR_Intron 7_Exon 8_R & & $\begin{array}{l}\text { ACATGGCTTCAGTGGACACAT } \\
\text { C }\end{array}$ \\
\hline QRSL1 QPCR_Exon 8_F & \begin{tabular}{l|} 
ACCAAAGGTCCTGAA \\
GTACAGATTा \\
\end{tabular} & \\
\hline QRSL1 QPCR_Exon 8_R & & АTTACTTTGGCCCCCTCAGACT \\
\hline QRSL1 QPCR_Exon 9_F & $\begin{array}{l}\text { GGCCTGATTITAGCC } \\
\text { AAAACTTTAC }\end{array}$ & \\
\hline QRSL1 QPCR_Exon 9_R & & $\begin{array}{l}\text { GAATTCTTCСТCTCACСАСАТC } \\
\text { A }\end{array}$ \\
\hline TBP QPCR_Exon 5_F & $\begin{array}{l}\text { CACGAACCACGGCAC } \\
\text { TGATT } \\
\end{array}$ & \\
\hline TBP QPCR_Exon 5_R & & TITCTTGCTGCCAGTCTGGAC \\
\hline
\end{tabular}




\section{Metabolic Complementation Experiments}

Human fibroblasts were grown in media containing either glucose or galactose as the main carbohydrate source and the acidification rate (ECAR) or the oxygen consumption rate (OCR) was determined with the Seahorse device (Seahorse Biosciences, Massachusetts, US), according to the protocol of the manufacturer. Fibroblasts of patient I.2.2 and a control were transduced with wild-type and mutant (without exon 8) cDNA, cloned in a QRSL1-pUltra-Chilli Lentiviral vector [9]. The results for mitochondrial respiration are normalized to control fibroblasts, transduced with wild-type cDNA.

\section{Results}

\section{Patient I}

For patient I.2.2 a total of 41,338 variants were detected in the WES data, of which 366 were homozygous variants mapping in the regions of homozygosity $(\mathrm{ROH})$. Variants that affected protein function were determined by in silico protein prediction tools, resulting in 24 of low frequency, damaging candidate variants (Supplementary table 4). The genes involved were prioritized for their likelihood of causing mitochondrial disease using Weighted Gene Expression Tool and database (WeGET). Based on this prioritization list we started our analysis with the QRSL1 gene, located in the ROH of chromosome 6, which carried a homozygous intronic variant A>G (NC_000006.11(NM_018292.4):c.850-3A>G (p.?), NP_060762.3), (Figure 2A). The QRSL1 variant possibly leads to abnormal splicing, based on 3 splicing predictions (Maximum Entropy Modeling of Short Sequence Motifs (MaxEnt): -100.0\%, Neural Networks Berkeley Drosophila Genome Project (NNSPLICE): $-98.6 \%$ and Human Splicing Finder (HSF): $-1.1 \%$ ), which showed that the normal splice acceptor site was slightly reduced and an acceptor site 3 bps downstream could be used. Functional splicing validation was required and segregation analysis showed that both affected siblings (I.2.1 and I.2.2) were homozygous for the variant. The parents (I.1.1 and I.1.2) were heterozygous, as was the unaffected brother (I.2.4). The unaffected sister (I.2.3) did not carry the variant (Figure 2A). 
PCR amplification of the QRSL1 CDNA using primers spanning the site of the variant, followed by sequence analysis revealed three different PCR fragments of 439bp, 330bp and 243bp for the mutant, whereas the wild type control cDNA showed only one band of 439bp (Figure 3A, B).

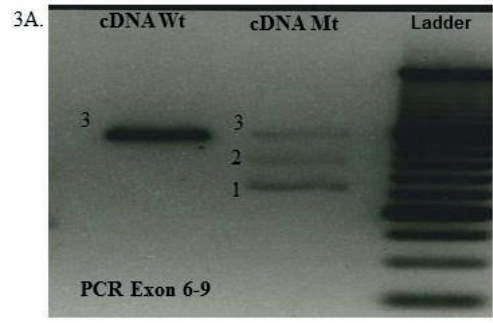

$3 \mathrm{C}$.

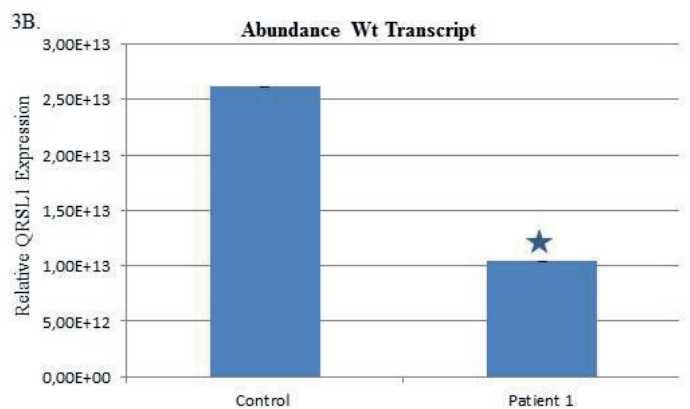

Control

Patient 1

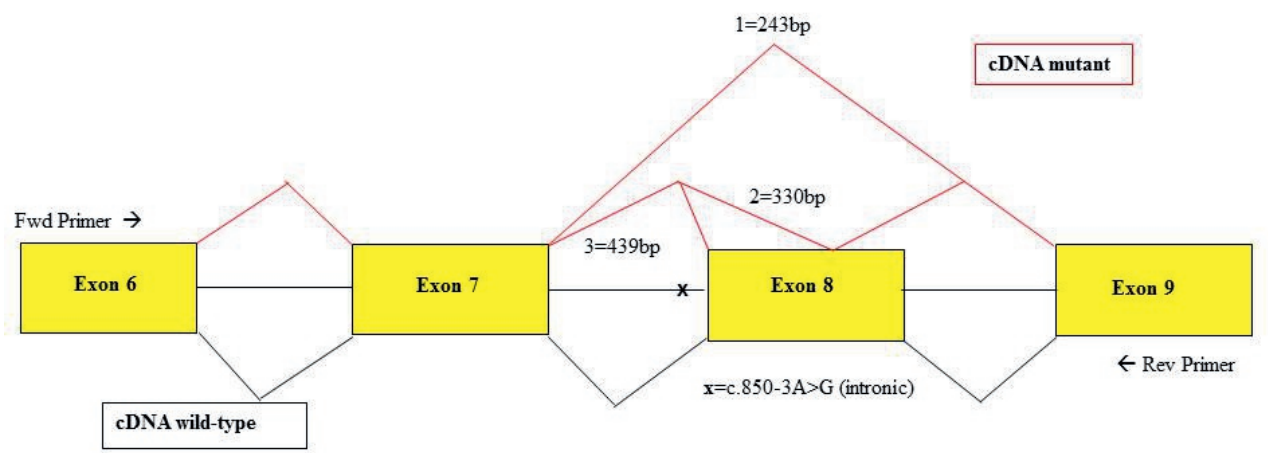

Figure 3. Analysis of splice defects in QRSL1 cDNA in patient I.2.2. 3A. QRLS1 CDNA of patient I.2.2 with variant A>G (NC_000006.11(NM_018292.4):C.850-3A>G) showed three CDNA fragments. 3B. Sequence analysis confirmed 3 different splicing events of intron 7 and 8 , resulting in fragments of $439 \mathrm{bp}$ (wild-type), $330 \mathrm{bp}$ and $243 \mathrm{bp}$. 3B. Percentage wild-type CDNA in patient I.2.2 compared to controls. The wild-type of patient I.2.2 cDNA (c.850$3 A>G)$ resulted in a wild-type significant rest $(*)$ level of $40 \%$ of the normal spliced cDNA in exon 8 ( $p<0.05$ by student T-test), three splice events for QRSL1 exon 8 in $3 C$.

Additional denaturing 10\% UREA PAGE gel confirmed the presence of 3 different fragments, excluding the possibility of one band being a hetero-duplex (Data not shown). Sequence analysis showed that in the PCR fragment of 243bp exon 8 was completely absent (NM_018292.4:r.850_1042del), leading to a frameshift and premature stop codon at exon 9. In the PCR fragment of 330bp, exon 8 was partly 
retained (37 amino acids of a total of 64 (NM_018292.4:r.850_931del), therefore missing 27 amino acids) and an exonic splice acceptor was used at position NM_018292.4:r.933 (c.933A). This also caused a frameshift and premature stop codon at position NM_018292.4:r.887* (c.887C). The 439bp fragment was the normally spliced cDNA fragment. Quantitative PCR analysis showed that wild-type cDNA was about $40 \%$ of the amount observed in control fibroblast (Figure $\mathbf{3 C}$ ).

Metabolic complementation measurements in fibroblasts of patient I.2.2 showed defects in glycolysis and OXPHOS. For a complementation test wild-type and mutant QRSL1 cDNAs was cloned in a pUltra-Chili Vector and subsequently used to transduce the fibroblasts of patient I.2.2 NDHF cells were negative controls. Patient cells transduced with wild-type cDNA QRSL1 showed a $61 \%$ OXPHOS activity ( $42 \%$ increase compared with non-transduced patient cells). The control cells showed a $51 \%$ OXPHOS activity when transduced with variant 1 (exon 8 was completely absent) cDNA QRSL1 (Figure 4). Polybrene was added to enhance the expression after transduction, but in addition to that a minor cytotoxic effect was observed in all cells for all conditions.

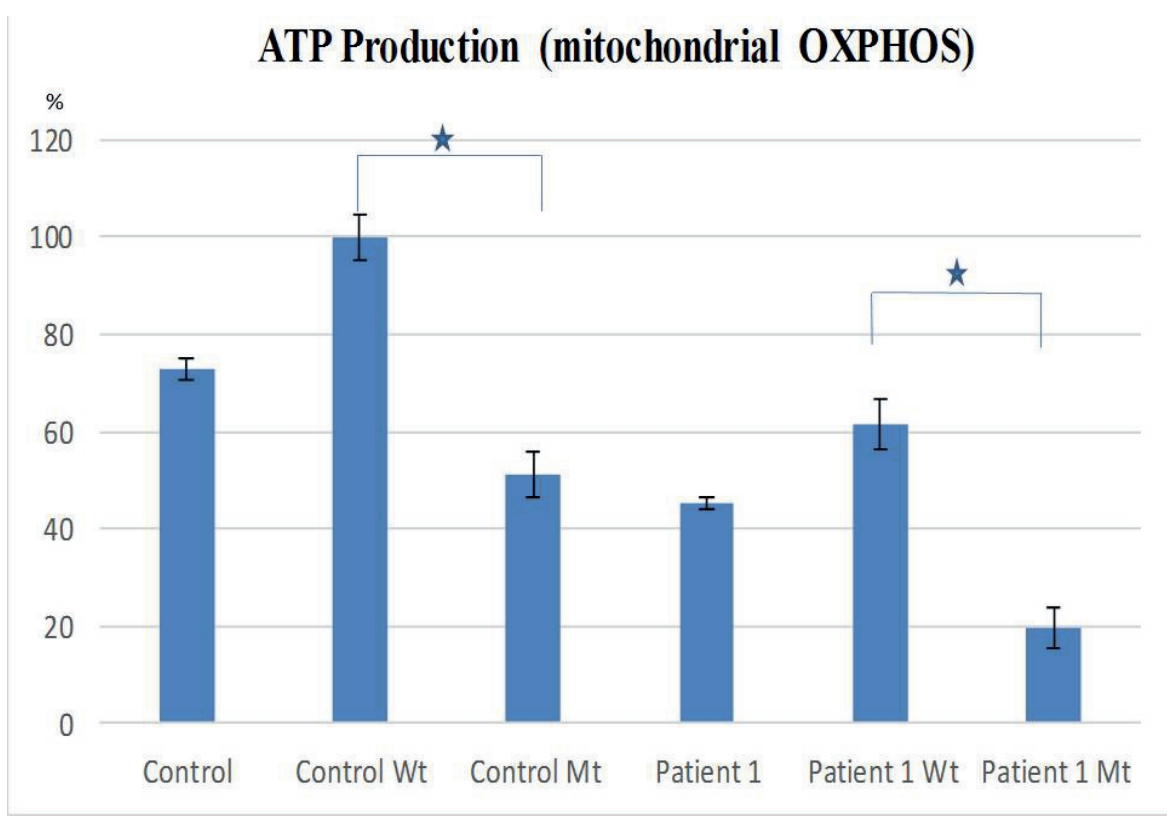

Figure 4. Complementation of QRSL1 defects in fibroblasts with 2 different vectors. Transduction with QRSL1 cDNA without exon 8 showed a significant decrease of $49 \%$ ATP 
production in control cells. A significant increase of 42\% ATP production in patient (I.2.2) and patient (I.2.2) plus transduced wild-type CDNA QRSL1 was observed. Both conditions were significant results $(p<0.05)$ by student $T$-test. The standard deviation was measured at four independent time points after transduction (Wild-type or mutant) and nontransduced (Control or Patient I.2.2). The results for mitochondrial respiration are normalized to control fibroblasts, transduced with wild-type cDNA.

\section{Patient II}

In patient II.2.1 we detected 4540 variants in the 412 mitochondrial genes, of which the MTO1 gene, containing two novel variants (NM_001123226.1, NP_001116698.1), Alanyl-tRNA Synthase 10, Supplementary table 5), was the most likely candidate based on function and genotype-phenotype correlation [16, 17]. The patient carried a G>A substitution (NM_001123226.1:C.253G>A) in exon 2, changing p.(G85R), and a G>A substitution (NM_001123226.1:c.938G>A) in exon 5, changing p.(R313Q), both of which were damaging by in silico predictions of Polyphen (probably damaging, score 0.95) and Sorting Intolerant From Tolerant (SIFT-deleterious, score 0.001). The first variant was not present in the in-house and ExAC database, whereas the second variant (rs number 371179032) had a very low allele frequency $(1 / 1211348, A F=8.241 E-6)$ in the ExAC database. Both variants were located in functional protein domains of MTO1, which were highly conserved, based on protein BioGraph annotated predictions. Each parent (II.1.1 and II.1.2) was heterozygous for one of the variants (Figure 2B).

\section{Patient III}

In patient III.2.1 we detected 4917 variants in the 412 mitochondrial genes, of which we identified two unknown variants and one known variant in the AARS2 (NM_020745.3, NP_065796.1, Alanyl-tRNA synthetase) gene (Supplementary table 6). Based on the HCM and Leigh-like phenotype, the AARS2 (Alanyl-tRNA synthetase) gene was the best candidate gene from the list (Supplementary table 6). The first variant was a G>A substitution (NM_020745.3:C.1774C>T) in exon 13 (rs138119149 with a frequency of 0.001), causing a substitution of p.(R592W) with a damaging score based on Polyphen (probably damaging score 0.97) and SIFT (deleterious score 0.002). The first variant was listed in ExAC: rs138119149 $(33 / 121110, A F=0.0002725)$. The second variant was a $G>A$ nonsense variant 
(NM_020745.3:C.2872C>T) in exon 22 had no rs number and was not present in the in-house or ExAC databases, p.(R958*). The unaffected father was heterozygous for the $\mathrm{G}>\mathrm{A}$ (c.1774C $>\mathrm{T}$ ) substitution in exon 13 and the unaffected mother was heterozygous for the nonsense variant c. $2872 \mathrm{C}>\mathrm{T}$ in exon 22 (Figure 2C).

\section{Discussion}

Cardiomyopathy is a disease of the heart muscle, interfering with efficient contraction and often leading to heart failure. According to the definition of the American Heart Association (AHA), cardiomyopathies can be either primary limited to the heart or can be a secondary result of other illnesses [10], Table 2. In 3 families with paediatric cardiomyopathy, brain disease, and multiple OXPHOS deficiencies we identified the causative variant by WES. Given the genetic heterogeneity of OXPHOS disorders with more than 1400 nuclear genes potentially involved, WES is the best suitable unbiased approach for finding the underlying genetic cause [11]. We started with an autosomal recessive disease model and in case of consanguinity as in patient I.2.2, we focused on homozygosity regions.

The second patient from a consanguineous marriage carried a homozygous splicing defect (NC_000006.11(NM_018292.4):c.850-3A>G, NP_060762.3) in the QRSL1 gene, leading to 2 mRNAs with a premature stop codon and a strong reduction of the normal mRNA. A complementation assay confirmed a causative role in the complex I deficiency and pathology. Therefore, we did not proceed with other candidate genes on the list. During the course of our studies a paper was published, reporting the first disease-causing variants in the QRSL1 gene. A homozygous variant (NM_018292.4:c.398G >T (p.(G133V)) was presented in a consanguineous family with two affected siblings (Patient $Y$ and $Z$ in Table 3 ) including hypertrophic cardiomyopathy (HCM) [12]. Also, a compound heterozygosity for the variants (NM_018292.4:c.350G>A (p.(G117E) and NM_018292.4:c.398G>T (p.(G133V)) was reported in another non-HCM patient (Patient AA in Table 3 ) with interstitial pneumonia and mitochondrial respiration deficiencies [12]. These data confirmed the involvement of QRSL1 in paediatric cardiomyopathy with OXPHOS deficiency. 


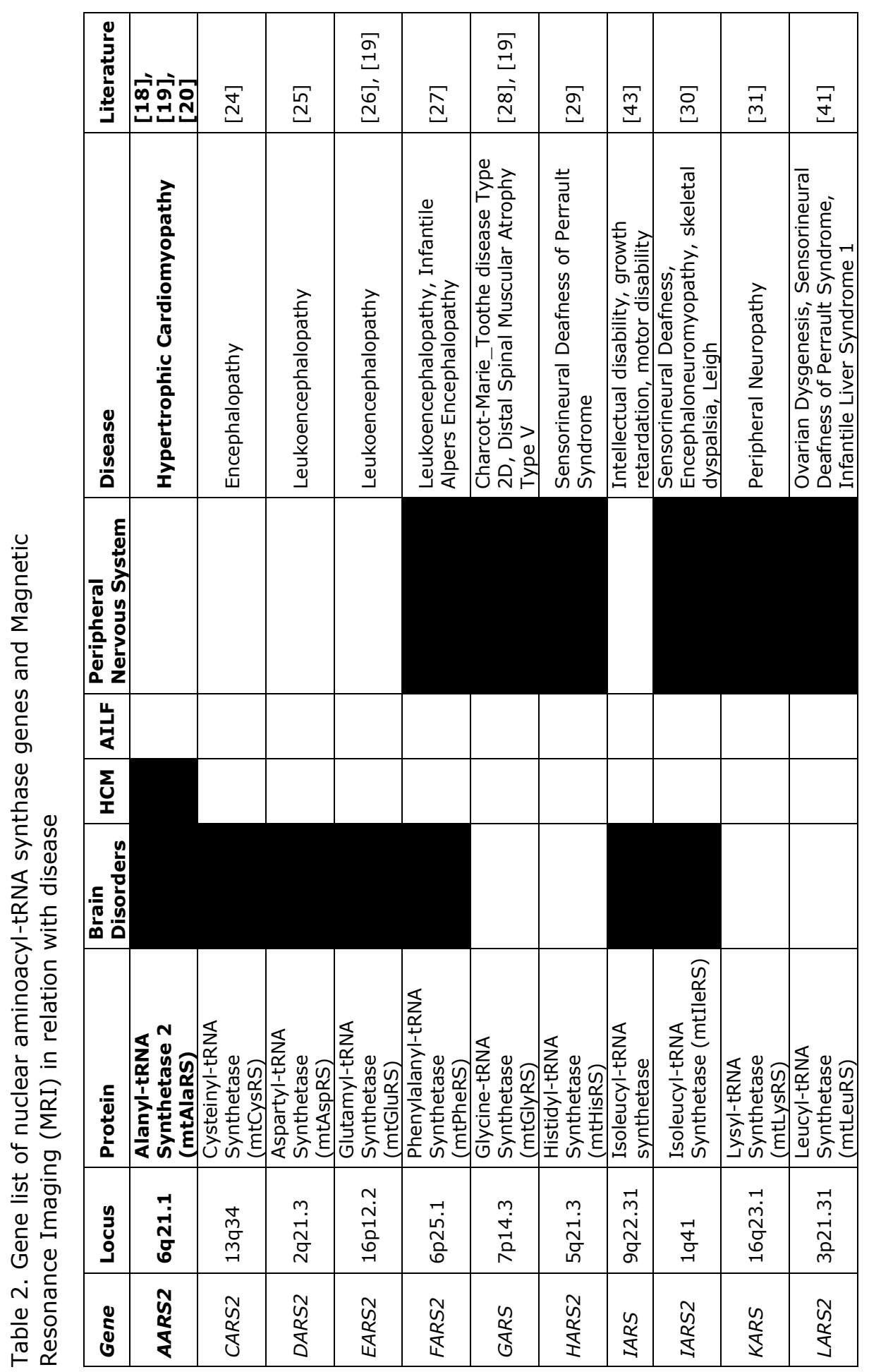




\begin{tabular}{|c|c|c|c|c|c|c|c|c|c|c|c|}
\hline 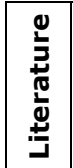 & $\stackrel{\widetilde{్}}{\sim}$ & 㝵 & $\begin{array}{l}\stackrel{\Xi}{\beth} \\
\stackrel{\Xi}{\Xi}\end{array}$ & 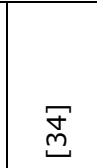 & $\underset{\mathbf{Z}}{\mathbf{Z}}$ & $\stackrel{\sqrt[n]{n}}{m^{2}}$ & 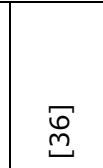 & $\stackrel{\sqrt{m}}{n}$ & $\begin{array}{l}\text { न } \\
\text { บ } \\
\dot{\infty} \\
\text { m }\end{array}$ & $\stackrel{\sqrt{m}}{\underline{m}}$ & 守 \\
\hline 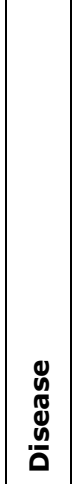 & 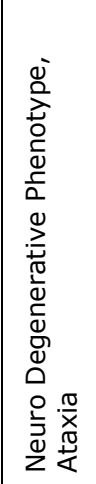 & 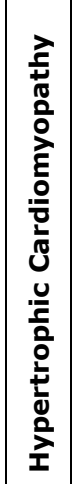 & 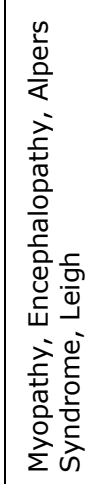 & 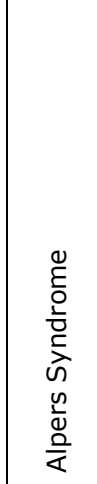 & 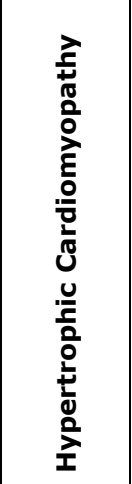 & 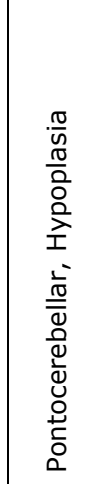 & 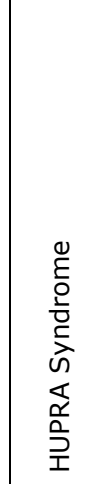 & 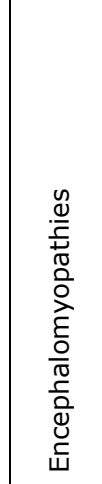 & 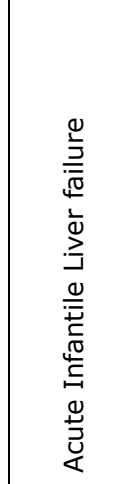 & 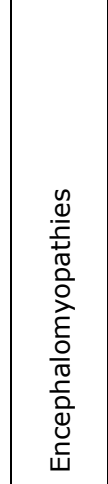 & 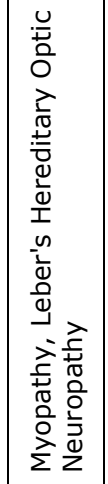 \\
\hline 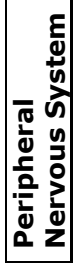 & & & & & & & & & & & \\
\hline 岁 & & & & & & & & & & & \\
\hline$\underset{\text { U }}{\Sigma}$ & & & & & & & & & & & \\
\hline r气 & & & & & & & & & & & \\
\hline 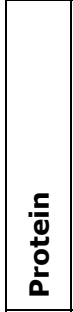 & 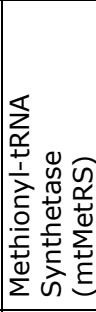 & 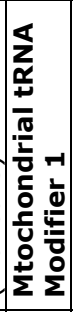 & 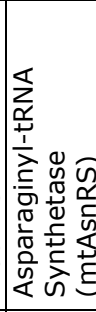 & 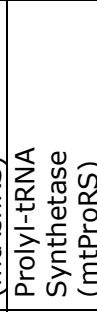 & 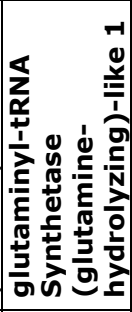 & 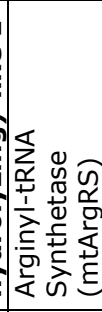 & 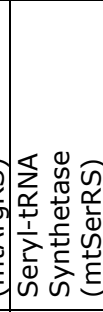 & 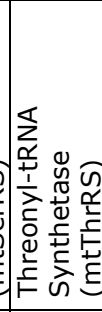 & 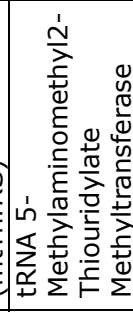 & 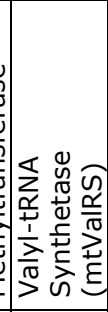 & 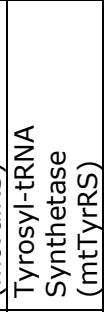 \\
\hline ב⿱艹 & $\begin{array}{l}\overrightarrow{-1} \\
\stackrel{m}{m} \\
\stackrel{\sim}{N}\end{array}$ & 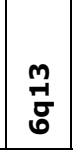 & 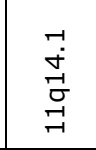 & 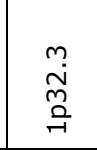 & 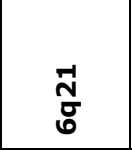 & $\begin{array}{l}\stackrel{n}{0} \\
\vec{\sigma} \\
\sigma\end{array}$ & $\begin{array}{l}\stackrel{N}{m} \\
\stackrel{m}{-1} \\
\stackrel{-}{\sigma} \\
\stackrel{-}{-1}\end{array}$ & 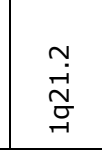 & $\begin{array}{l}\vec{m} \\
\stackrel{m}{ } \\
\overrightarrow{\tilde{N}} \\
\stackrel{N}{N}\end{array}$ & $\begin{array}{l}\stackrel{m}{m} \\
\stackrel{-}{N} \\
\stackrel{0}{0}\end{array}$ & $\begin{array}{l}\stackrel{-}{N} \\
-i \\
\stackrel{-}{n} \\
\underset{\sim}{+}\end{array}$ \\
\hline Ð & $\begin{array}{l}\text { N } \\
\text { रू } \\
\Sigma \\
\Sigma\end{array}$ & $\sum_{\Sigma}^{n}$ & 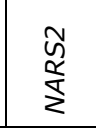 & 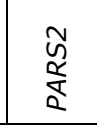 & \begin{tabular}{l} 
ज̃ \\
\multirow{\sigma}{*}{}
\end{tabular} & $\begin{array}{l}\text { N } \\
\frac{1}{2} \\
\frac{1}{2}\end{array}$ & $\begin{array}{l}\text { जे } \\
\text { of } \\
\text { जे }\end{array}$ & $\begin{array}{l}\text { N } \\
\text { 足 } \\
\text { F }\end{array}$ & $\underset{\sum}{\stackrel{\aleph}{k}}$ & $\begin{array}{l}\text { vै } \\
\text { रू } \\
\frac{1}{5}\end{array}$ & $\begin{array}{l}\text { N } \\
\frac{2}{\Sigma} \\
\frac{1}{2}\end{array}$ \\
\hline
\end{tabular}




\begin{tabular}{|c|c|c|c|c|c|c|c|c|c|c|c|c|c|c|c|c|c|}
\hline 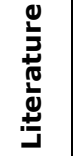 & & $\stackrel{\infty}{\infty}$ & $\stackrel{\infty}{\Xi}$ & & 号 & & & ન્ન & & 亏 & & 온 & & ్ㅗㄴ & & 몬 & \\
\hline 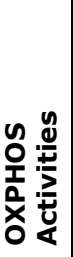 & & 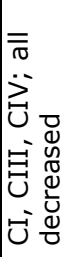 & 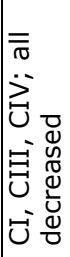 & & 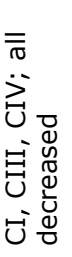 & 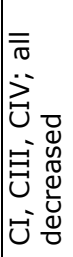 & & 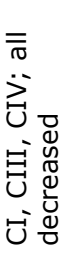 & & 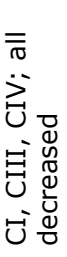 & & 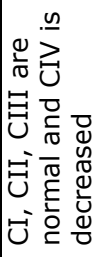 & & & & & \\
\hline 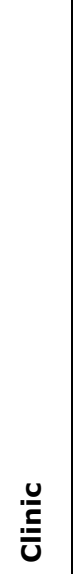 & & 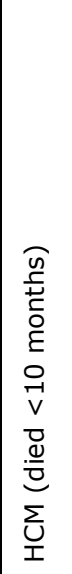 & 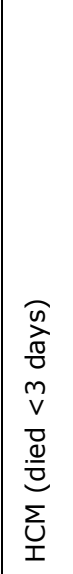 & & 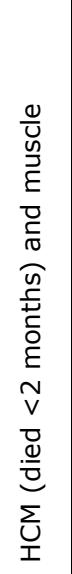 & 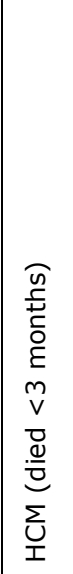 & & 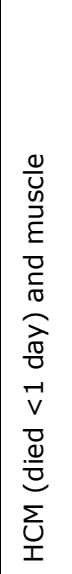 & & 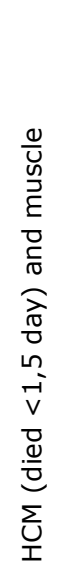 & & 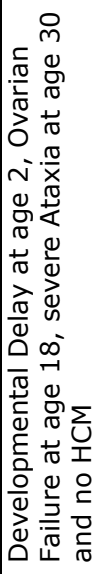 & & 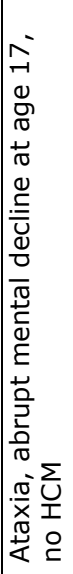 & & 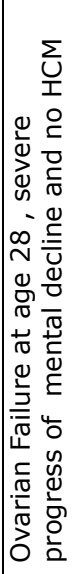 & \\
\hline 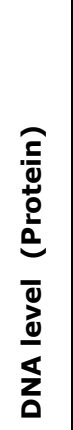 & & 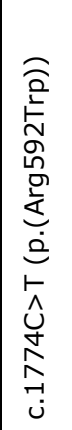 & 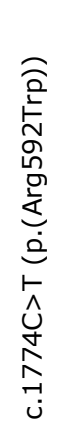 & 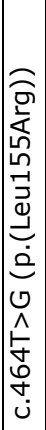 & 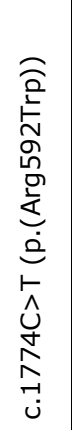 & 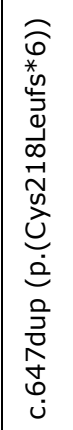 & 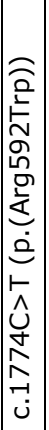 & 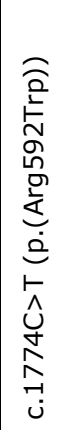 & 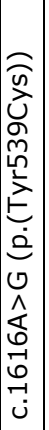 & 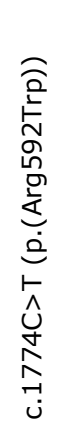 & 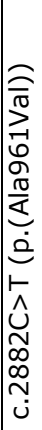 & 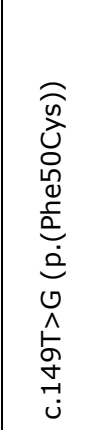 & 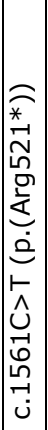 & 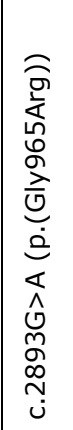 & 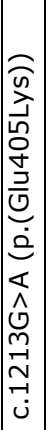 & 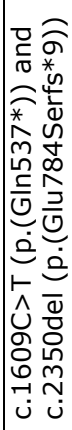 & 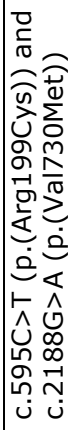 \\
\hline 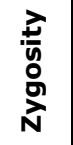 & & $\stackrel{N}{I}$ & 蒫 & 芒 & $\stackrel{N}{I}$ & 蒫 & 포 & 蒫 & 茥 & 蒫 & 茥 & 茥 & 폰 & 蒫 & 童 & 포 & 蒫 \\
\hline 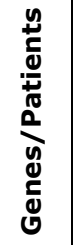 & \begin{tabular}{l}
\multirow{2}{*}{} \\
$\frac{\alpha}{\alpha}$
\end{tabular} & 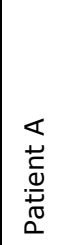 & 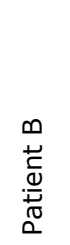 & & 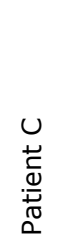 & 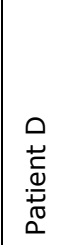 & & 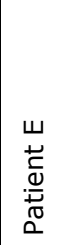 & & 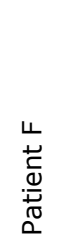 & & 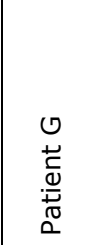 & & 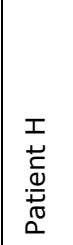 & & 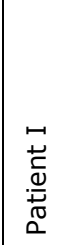 & \\
\hline
\end{tabular}




\begin{tabular}{|c|c|c|c|c|c|c|c|c|c|c|c|c|c|c|c|c|c|}
\hline 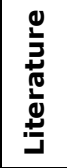 & ్ㅗㅁ & & 实 & & 穴 & & 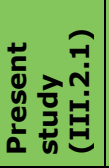 & & 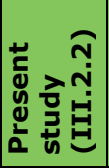 & & 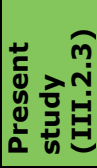 & & & $\underset{\square}{\square}$ & & $\underset{\varpi}{\varpi}$ & \\
\hline 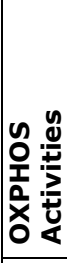 & & & & & 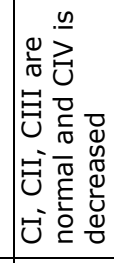 & & 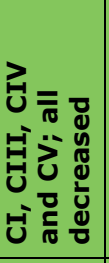 & & 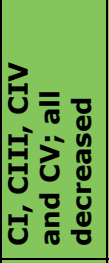 & & 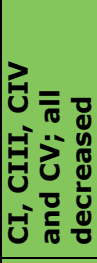 & & & 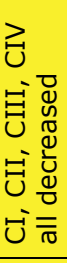 & & 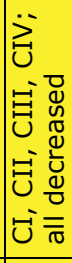 & \\
\hline$\frac{u}{\underline{E}}$ & 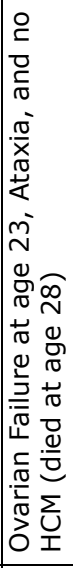 & & 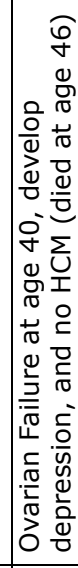 & & 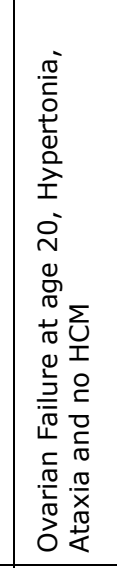 & & 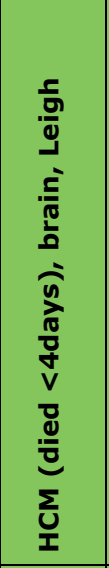 & & 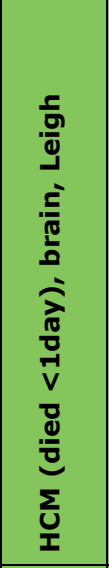 & & 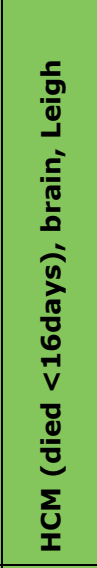 & & & 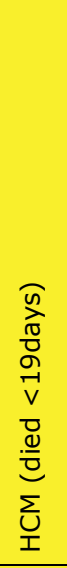 & & 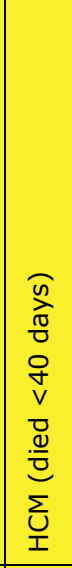 & \\
\hline 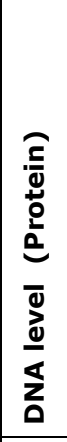 & 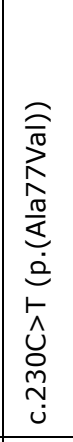 & 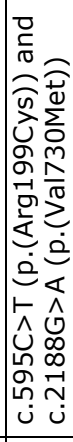 & 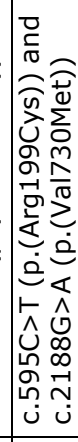 & 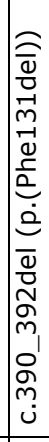 & 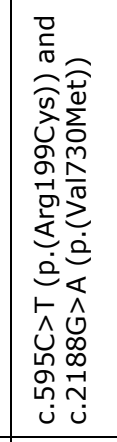 & 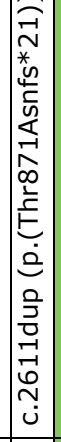 & 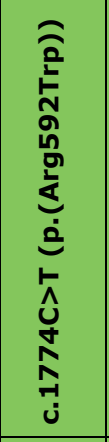 & 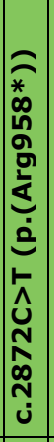 & 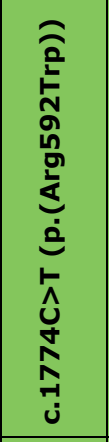 & 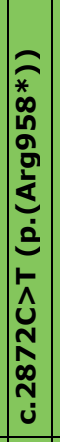 & 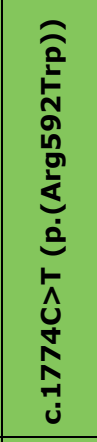 & 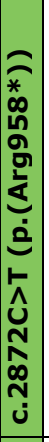 & & 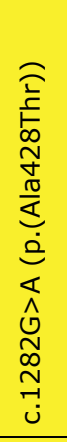 & 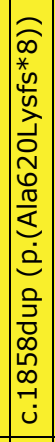 & 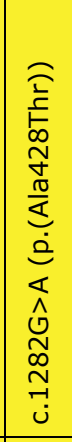 & 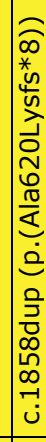 \\
\hline $\begin{array}{l}3 \\
\frac{1}{n} \\
0 \\
0 \\
\text { ते }\end{array}$ & 茎 & 포 & 포 & 苀 & 茎 & 芒 & 茥 & 芏 & 茥 & \pm & 茥 & 茥 & & 蒫 & 茥 & 茎 & 芒 \\
\hline 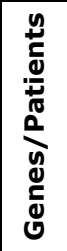 & 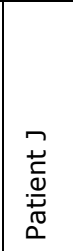 & & 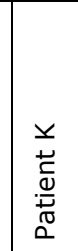 & & 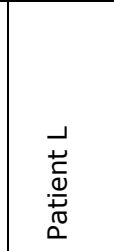 & & 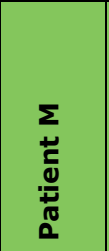 & & 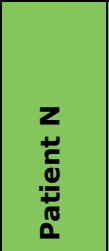 & & 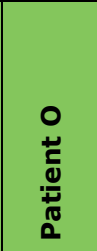 & & ¿-1 & 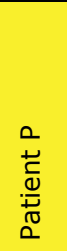 & & 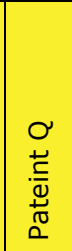 & \\
\hline
\end{tabular}




\begin{tabular}{|c|c|c|c|c|c|c|c|c|c|c|c|c|c|c|c|c|}
\hline 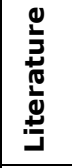 & $\stackrel{\square}{\square}$ & 글 & & ㅋ & ন & స & ㅋ. & 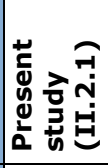 & & & $\underset{\beth}{\beth}$ & $\underset{\beth}{\beth}$ & $\stackrel{\underset{N}{\beth}}{\Xi}$ & & 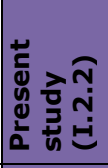 & 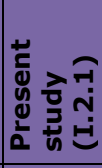 \\
\hline 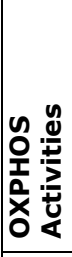 & 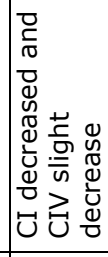 & 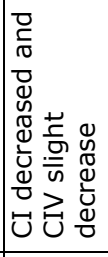 & & 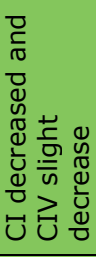 & ¿ே. & 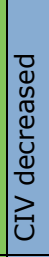 & 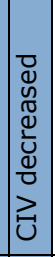 & 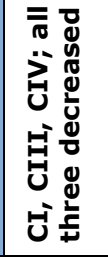 & & & 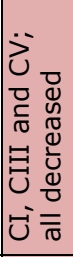 & . & 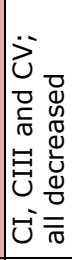 & & 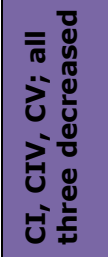 & ர் \\
\hline$\frac{u}{\frac{s}{\sigma}}$ & 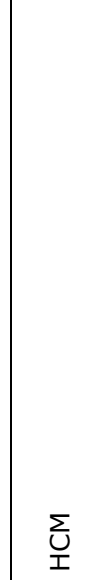 & 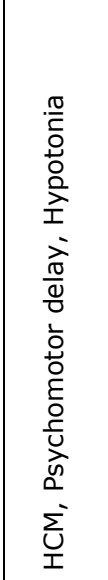 & & 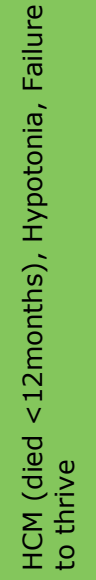 & 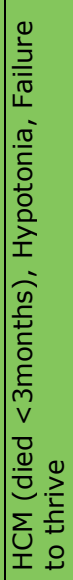 & 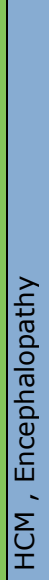 & 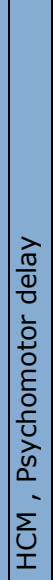 & 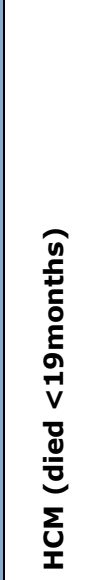 & & & 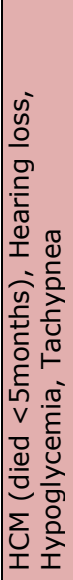 & 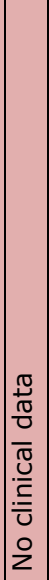 & 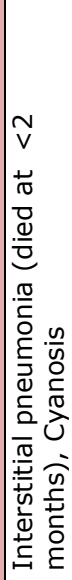 & & 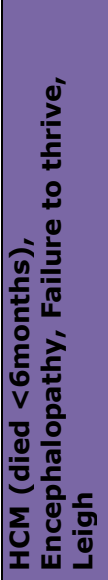 & 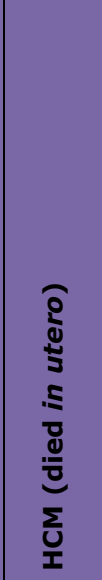 \\
\hline 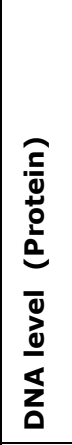 & 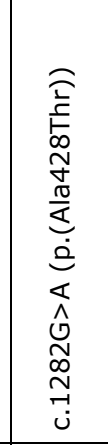 & 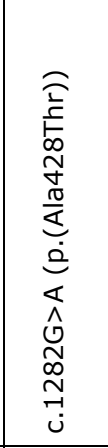 & 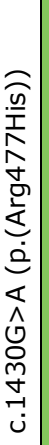 & 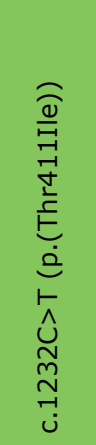 & 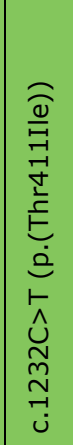 & 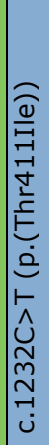 & 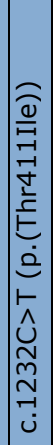 & 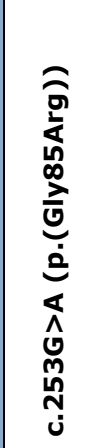 & 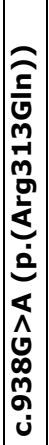 & & 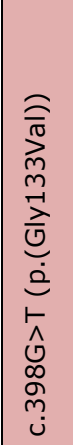 & 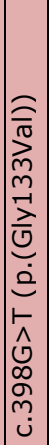 & 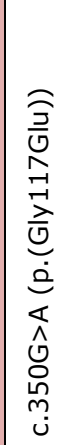 & 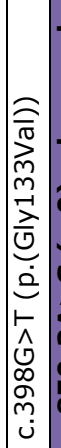 & 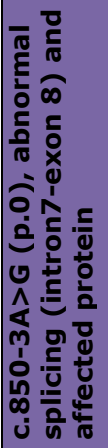 & 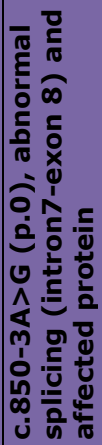 \\
\hline $\begin{array}{l}\frac{2}{5} \\
\frac{0}{0} \\
\frac{0}{N}\end{array}$ & N & 茎 & 포 & $\stackrel{N}{I}$ & $\stackrel{N}{I}$ & $\stackrel{N}{I}$ & $\mid \mathbf{N}$ & 茥 & 芒 & & $\stackrel{N}{I}$ & $\stackrel{N}{I}$ & 茥 & 茎 & I & $\mathbf{N}$ \\
\hline 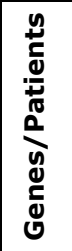 & 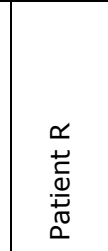 & $\begin{array}{l}u \\
+1 \\
\frac{1}{d} \\
\stackrel{D}{0} \\
0\end{array}$ & & 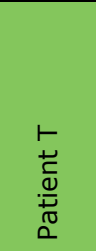 & 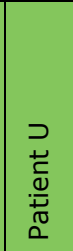 & 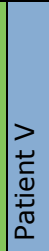 & 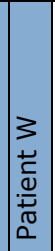 & 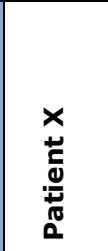 & & $\mid \begin{array}{l}\vec{y} \\
\bar{u} \\
\alpha \\
\alpha\end{array}$ & 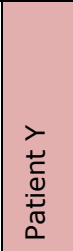 & 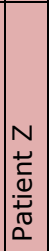 & 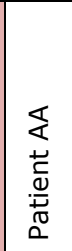 & & 宸 & 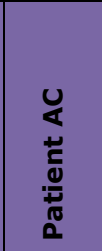 \\
\hline
\end{tabular}




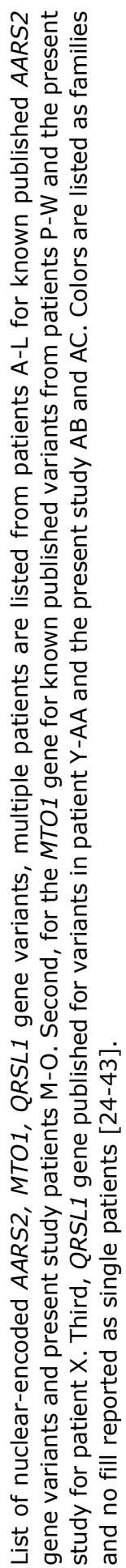


The clinical manifestations vary, and based on the limited data, a strong red uction in QRSL1 seems more severe than the amino acid changes reported, although data on the brain phenotype are lacking (Tables 2, 3). QRSL1 (or GatA) is part of a heterotrimeric enzyme complex GatCAB; GatA, GatB (PET112L), and GatC (GATC). GatA (QRSL1) plays a role as a mitochondrial amido-transferase, recognizing Glu-tRNA (GIn) and converts Glu to Gln. An accumulation of Glu-tRNA previously showed defects of mitochondrial mammalian translation processes and respiration [13]. The QRSL1 defect can explain the observed defects in glycolysis and OXPHOS. This is supported by earlier data on the role of mitochondrial translation and glutaminyl-mt tRNA ${ }^{\text {Gln }}$ in mouse cells, which demonstrated that mgatA produced a strong mitochondrial translational aminoacylation defect [14]. In Drosophila, null mutants of GatA gene, the homolog of QRSL1, caused larvae to grow slowly and die before pupariation [15]. As one (I.2.1) of the siblings of family 1 died in utero this could reflect a similar developmental defect. Both studies support the essential role for glutaminyl-mt tRNA ${ }^{\text {Gln }}$ in mitochondrial protein synthesis and OXPHOS.

In patient II.2.1 we identified two novel variants in the MTO1 gene, which is involved in mitochondrial tRNA modification. MTO1 catalyzes the 5carboxymethylaminomethylation of the uridine base in mitochondrial tRNA-GIn, tRNA-Glu, and tRNA-Lys, usually together with MTO2 (TRMU). A disease-causing role of MTO1 in mitochondrial patients with hypertrophic cardiomyopathy (Table 2, 3) has been described before [16, 17]. The two variants (NM_012123.3:c.1282G>A (p.(Ala428Thr) and NM_012123.3:c.1858dup (p.(Ala620Lysfs*8)) in siblings patients $P$ and $Q$ showed early-onset lethal HCM, but normal brain anatomy (Table 3). An unrelated third consanguineous patient R with NM_012123.3:c.1282G>A (p.(Ala428Thr) showed HCM without lethality, and also normal brain development. In two consanguineous families (lethal male siblings $T / U$ and non-lethal female siblings $V / W$ ) a homozygous variant (NM_012123.3:c.1232C>T (p.(Thr411Ile)) was reported causing early-onset HCM including abnormal brain morphology by MRI. Our two novel variants in MTO1 gene in patient II.2.1 (Table 3, patient X) displayed a similar early-onset HCM, a diffuse cerebral atrophy with an intraventricular septa, and multiple OXPHOS deficiencies, and lethality at 19 months. 
In patients III.2.1, III.2.2 and III.2.3 we identified two variants in the AARS2 gene: NM_020745.3:c.1774C>T (p.(R592W) , and NM_020745.3:c.2872C>T (p.(R958*). AARS2 consists of three functional domains; the N-terminal catalytic domain, the Editing domain and the C-terminal (C-Ala domain). The AARS2 gene belongs to the class-II aminoacyl-tRNA synthase family and the protein is functional as a dimer. The first variant (c.1774C $>\mathrm{T}$ ) on the paternal allele are in the editing domain of the AARS2 gene. The editing domain removes incorrectly charged amino acids and the first (c.1774C $>\mathrm{T}$ ) variant is previously reported as a disease-causing variant associated with $\mathrm{HCM}$ and a poor prognosis $[18,19]$. The second novel stop (c.2872C $>$ T) variant on the maternal allele is located in the cterminus (C-Ala domain) for the AARS2 gene and most likely leads to nonsensemediated decay of the transcript. The presence of a reduced amount of the protein and the presence of 2 detrimental amino acid substitutions in the other allele could, especially in dimers like AARS2, explain the more severe disease manifestation, of paediatric cardiomyopathy, Leigh-like syndrome, and multiple OXPHOS deficiencies. One of the patients in literature (patient D in Table 3 ) was compound heterozygous for the same missense mutation in combination with a frameshift mutation (NM_020745.3:c.[647dup];[1774C>T]), (p.[(C218Lfs*6)];[(R592W)]). The frameshift variant (p.(C218Lfs*6)) probably leads to nonsense-mediated decay of the transcript. Both variants resulted in cardiomyopathy and lethality after 3 months of birth [19-21].

Different variants affecting protein function have been reported for the AARS2 gene resulting in $\mathrm{HCM}$, leukodystrophy, and ovarian failure $[20,21]$ and a genotype-phenotype correlation is needed for novel variants in genes. Structural modeling of tissue-specific mitochondrial alanyl-tRNA synthetase (AARS2) defects was previously reported to predict different effects on aminoacylation activity level for the protein [21]. The study showed a clear correlation between the amino acid change ( $p .(R 592 W))$ and the severity of the predicted aminoacylation activity of AARS2 linked to cardiomyopathy. In case of a significant residual amount of mischarged aminoacids, the predicted activity of aminoacylation resulted in a brain disease phenotype. Strikingly, none of the missense variants in leukodystrophy patients were reported in the editing domain in contrast to the cardiomyopathy patients [21]. Nevertheless, several missense variants were 
described outside the editing domain (N-terminal catalytic and C-terminal (C-Ala domain) and caused a cardiomyopathy phenotype $[18,19,21]$. Further insights in protein modeling and in vivo studies (e.g. mice or zebrafish) can further elucidate the role of translation defects during mitochondrial protein synthesis.

In all three patients, paediatric cardiomyopathy, early-onset brain disease, and multiple OXPHOS deficiencies were present. Our data, together with other reports, indicates that the heart is sensitive to severe defects in mitochondrial translation during prenatal development (Table 2). The heart muscle consists for $30 \%$ of mitochondria, which have a critical role in the structure alignment of heart muscle fibres and, in supplying the heart with sufficient energy. Adequate mitochondrial translation is essential for both processes, especially during development when the heart differentiates from early embryonic cells by massive proliferation of the mitochondria. It is therefore likely that the disturbed translation causes structural problems (hypertrophic cardiomyopathy) in combination with OXPHOS defects in the prenatal and neonatal heart. Evidence supporting this hypothesis can be derived from other cardiomyopathy disease causing variants in mitochondrial translation, like the tRNAGly NC_012920.1:m.9997T>C, tRNAHis NC_012920.1:m.12192G>A， tRNALys NC_012920.1:m.8344A>G， tRNALeu NC_012920.1:m.3243A>G and NC_012920.1:m.3302A>G, tRNAIle NC_012920.1:m.4317A>G, tRNATrp NC_012920.1:m.5545C>T, and tRNAVal NC_012920.1:m.1644G>A. Furthermore, other defects in nuclear genes involved in mitochondrial protein synthesis are reported to cause pediactric cardiomyopathy e.g. mitochondrial ribosomal subunits (MRPL3, MRPL44), tRNAmodifiers (GTPBP3), and RNA processing enzyme (ELAC2) [21, 22]. Cardiomyopathy can be the sole manifestation, but it can also be part of a broader syndromic spectrum. However, not all defects in genes involved in mitochondrial translation lead to hypertrophic cardiomyopathy, as apart from the AARS2 gene none of the tRNA synthetases genes affect the heart, but rather the brain, liver, and peripheral nervous system (Table 2 ). The number of variants involved in translation of mitochondrial proteins, is yet too low to define whether this is genespecific or variant-specific, except for the AARS2 gene where a genotypephenotype correlation has been established. 


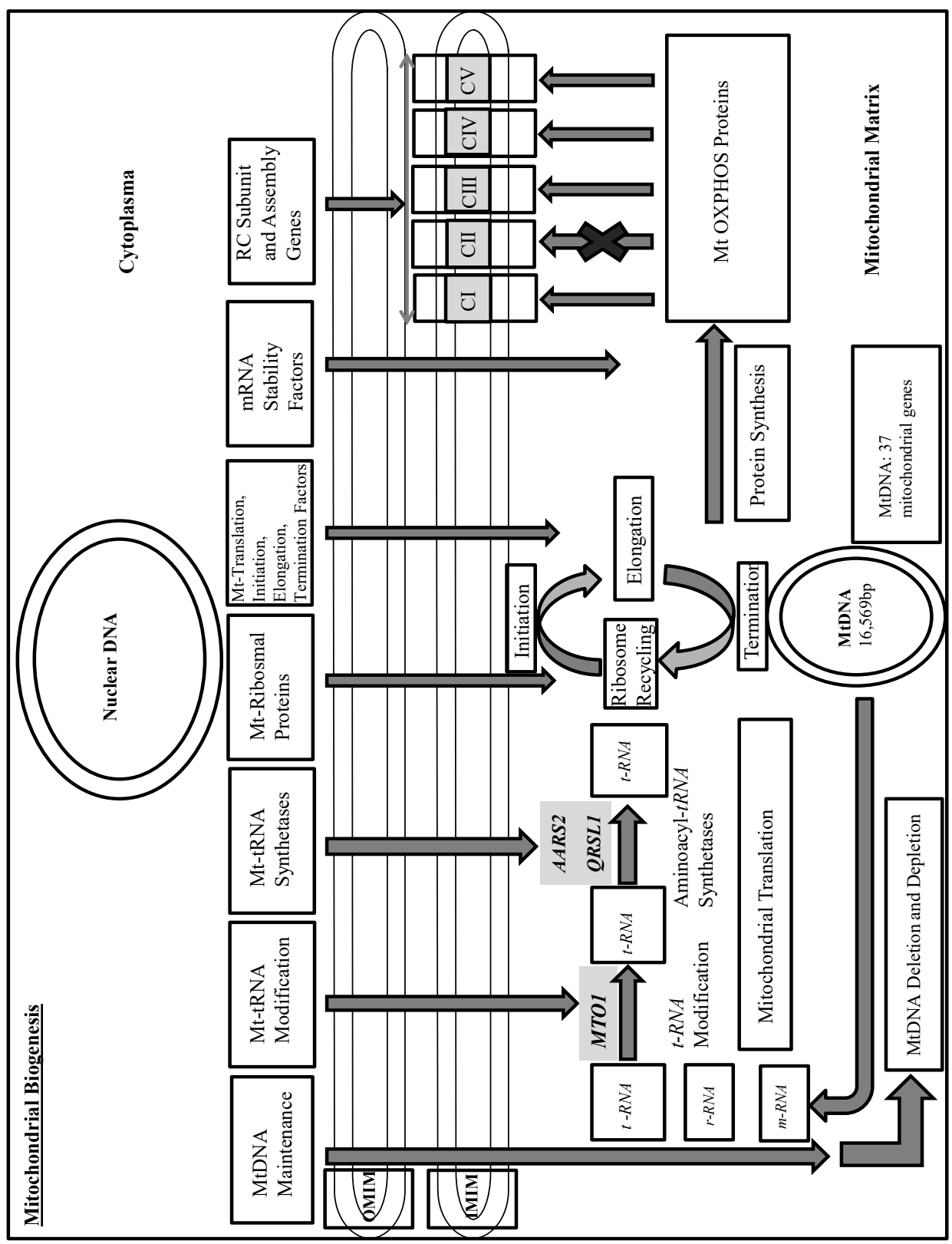

Figure 5. Mitochondrial Biogenesis. A schematic overview of the pathways involved in mitochondrial biogenesis, including the translation processes, affected in our patients. The reported genes are highlighted. 
In conclusion, our study showed disease-causing variants in the nuclear aminoacyl-tRNA synthetase genes (AARS2, MTO1, QRSL1), causing paediatric cardiomyopathy, brain, and multiple OXPHOS deficiencies (Figure 5). As the expression can be variable, it would be worthwhile to investigate the brain, even if the patient only presents with cardiomyopathy, as this is important for prognosis. Our data are in line with a key role of mitochondria in cardiovascular genetics and disease [23].

\section{Acknowledgements}

The authors would like to acknowledge the patients and their families for their contribution to this study. Elvira N. Mulder-Den Hartog (Erasmus MC, Rotterdam) for clinical information, and Ralph W. Gottschalk (MUMC+, Maastricht) for the diagnostic analyses. This work was supported by a grant from the Princes Beatrix Spierfonds (W.OR11-24) and Metakids.

\section{Conflict of Interest}

Rick Kamps, Radek Szklarczyk, Tom Theunissen, Debby M.E.I. Hellebrekers, Suzanne C.E.H. Sallevelt, Iris Boesten, Bart de Koning, Bianca van den Bosch, Gajja S. Salomons, Marisa Simas-Mendes, Rob Verdijk, Kees Schoonderwoerd, Irenaeus F.M. de Coo, Jo Vanoevelen and Hubert J.M. Smeets declare that they have no conflict of interest. All procedures followed were in accordance with the ethical standards of the Helsinki Declaration of 1975, as revised in 2000. 


\section{References}

1. Gorman, G.S., et al., Mitochondrial diseases. Nat Rev Dis Primers, 2016. 2: p. 16080.

2. Konovalova, S. and H. Tyynismaa, Mitochondrial aminoacyl-tRNA synthetases in human disease. Mol Genet Metab, 2013. 108(4): p. 20611.

3. Klinische Genetica. Gene-panel Mitochondria, . 2019, 2 August [cited 2019 2 August]; Available from: https://www.labmaastricht.nl/klinischegenetica.

4. Gohil, V.M., et al., Nutrient-sensitized screening for drugs that shift energy metabolism from mitochondrial respiration to glycolysis. Nat Biotechnol, 2010. 28(3): p. 249-55.

5. Gerards, M., et al., Exome sequencing reveals a novel Moroccan founder mutation in SLC19A3 as a new cause of early-childhood fatal Leigh syndrome. Brain, 2013. 136(Pt 3): p. 882-90.

6. Genomes Project, C., et al., A global reference for human genetic variation. Nature, 2015. 526(7571): p. 68-74.

7. Seelow, D., et al., HomozygosityMapper--an interactive approach to homozygosity mapping. Nucleic Acids Res, 2009. 37(Web Server issue): p. W593-9.

8. Ohtake, A., et al., Diagnosis and molecular basis of mitochondrial respiratory chain disorders: exome sequencing for disease gene identification. Biochim Biophys Acta, 2014. 1840(4): p. 1355-9.

9. Lou, E., et al., Tunneling nanotubes provide a unique conduit for intercellular transfer of cellular contents in human malignant pleural mesothelioma. PLoS One, 2012. 7(3): p. e33093.

10. Maron, B.J., et al., Prevalence of hypertrophic cardiomyopathy in a general population of young adults. Echocardiographic analysis of 4111 subjects in the CARDIA Study. Coronary Artery Risk Development in (Young) Adults. Circulation, 1995. 92(4): p. 785-9.

11. Calvo, S.E. and V.K. Mootha, The mitochondrial proteome and human disease. Annu Rev Genomics Hum Genet, 2010. 11: p. 25-44.

12. Kohda, M., et al., A Comprehensive Genomic Analysis Reveals the Genetic Landscape of Mitochondrial Respiratory Chain Complex Deficiencies. PLoS Genet, 2016. 12(1): p. e1005679.

13. Suzuki, T., A. Nagao, and T. Suzuki, Human mitochondrial tRNAs: biogenesis, function, structural aspects, and diseases. Annu Rev Genet, 2011. 45: p. 299-329.

14. Echevarria, L., et al., Glutamyl-tRNAGIn amidotransferase is essential for mammalian mitochondrial translation in vivo. Biochem J, 2014. 460(1): p. 91-101.

15. Nagao, A., et al., Biogenesis of glutaminyl-mt tRNAGln in human mitochondria. Proc Natl Acad Sci U S A, 2009. 106(38): p. 16209-14. 
16. Ghezzi, D., et al., Mutations of the mitochondrial-tRNA modifier MTO1 cause hypertrophic cardiomyopathy and lactic acidosis. Am J Hum Genet, 2012. 90(6): p. 1079-87.

17. Baruffini, E., et al., MTO1 mutations are associated with hypertrophic cardiomyopathy and lactic acidosis and cause respiratory chain deficiency in humans and yeast. Hum Mutat, 2013. 34(11): p. 1501-9.

18. Gotz, A., et al., Exome Sequencing Identifies Mitochondrial Alanyl-tRNA Synthetase Mutations in Infantile Mitochondrial Cardiomyopathy. American Journal of Human Genetics, 2011. 88(5): p. 635-642.

19. Taylor, R.W., et al., Use of Whole-Exome Sequencing to Determine the Genetic Basis of Multiple Mitochondrial Respiratory Chain Complex Deficiencies. Jama-Journal of the American Medical Association, 2014. 312(1): p. 68-77.

20. Dallabona, C., et al., Novel (ovario) leukodystrophy related to AARS2 mutations. Neurology, 2014. 82(23): p. 2063-71.

21. Euro, L., et al., Structural modeling of tissue-specific mitochondrial alanyltRNA synthetase (AARS2) defects predicts differential effects on aminoacylation. Front Genet, 2015. 6: p. 21.

22. Liu, Y., et al., Deficiencies in tRNA synthetase editing activity cause cardioproteinopathy. Proceedings of the National Academy of Sciences of the United States of America, 2014. 111(49): p. 17570-17575.

23. Murphy, E., et al., Mitochondrial Function, Biology, and Role in Disease: $A$ Scientific Statement From the American Heart Association. Circ Res, 2016. 118(12): p. 1960-91.

24. Coughlin, C.R., 2nd, et al., Mutations in the mitochondrial cysteinyl-tRNA synthase gene, CARS2, lead to a severe epileptic encephalopathy and complex movement disorder. J Med Genet, 2015. 52(8): p. 532-40.

25. Taft, R.J., et al., Mutations in DARS cause hypomyelination with brain stem and spinal cord involvement and leg spasticity. Am J Hum Genet, 2013. 92(5): p. 774-80.

26. Steenweg, M.E., et al., Leukoencephalopathy with thalamus and brainstem involvement and high lactate 'LTBL' caused by EARS2 mutations. Brain, 2012. 135(Pt 5): p. 1387-94.

27. Elo, J.M., et al., Mitochondrial phenylalanyl-tRNA synthetase mutations underlie fatal infantile Alpers encephalopathy. Hum Mol Genet, 2012. 21(20): p. 4521-9.

28. Antonellis, A., et al., Glycyl tRNA synthetase mutations in Charcot-MarieTooth disease type 2D and distal spinal muscular atrophy type $V$. Am J Hum Genet, 2003. 72(5): p. 1293-9.

29. Pierce, S.B., et al., Mutations in mitochondrial histidyl tRNA synthetase HARS2 cause ovarian dysgenesis and sensorineural hearing loss of Perrault syndrome. Proc Natl Acad Sci U S A, 2011. 108(16): p. 6543-8.

30. Schwartzentruber, J., et al., Mutation in the nuclear-encoded mitochondrial isoleucyl-tRNA synthetase IARS2 in patients with cataracts, growth hormone deficiency with short stature, partial sensorineural 
deafness, and peripheral neuropathy or with Leigh syndrome. Hum Mutat, 2014. 35(11): p. 1285-9.

31. McLaughlin, H.M., et al., Compound heterozygosity for loss-of-function Iysyl-tRNA synthetase mutations in a patient with peripheral neuropathy. Am J Hum Genet, 2010. 87(4): p. 560-6.

32. Bayat, V., et al., Mutations in the mitochondrial methionyl-tRNA synthetase cause a neurodegenerative phenotype in flies and a recessive ataxia (ARSAL) in humans. PLoS Biol, 2012. 10(3): p. e1001288.

33. Vanlander, A.V., et al., Two siblings with homozygous pathogenic splicesite variant in mitochondrial asparaginyl-tRNA synthetase (NARS2). Hum Mutat, 2015. 36(2): p. 222-31.

34. Sofou, K., et al., Whole exome sequencing reveals mutations in NARS2 and PARS2, encoding the mitochondrial asparaginyl-tRNA synthetase and prolyl-tRNA synthetase, in patients with Alpers syndrome. Mol Genet Genomic Med, 2015. 3(1): p. 59-68.

35. Edvardson, S., et al., Deleterious mutation in the mitochondrial arginyltransfer RNA synthetase gene is associated with pontocerebellar hypoplasia. Am J Hum Genet, 2007. 81(4): p. 857-62.

36. Belostotsky, R., et al., Mutations in the mitochondrial seryl-tRNA synthetase cause hyperuricemia, pulmonary hypertension, renal failure in infancy and alkalosis, HUPRA syndrome. Am J Hum Genet, 2011. 88(2): p. 193-200.

37. Diodato, D., et al., VARS2 and TARS2 mutations in patients with mitochondrial encephalomyopathies. Hum Mutat, 2014. 35(8): p. 983-9.

38. Yan, Q., et al., Human TRMU encoding the mitochondrial 5methylaminomethyl-2-thiouridylate-methyltransferase is a putative nuclear modifier gene for the phenotypic expression of the deafnessassociated 12S rRNA mutations. Biochem Biophys Res Commun, 2006. 342(4): p. 1130-6.

39. Zeharia, A., et al., Acute infantile liver failure due to mutations in the TRMU gene. Am J Hum Genet, 2009. 85(3): p. 401-7.

40. Riley, L.G., et al., Mutation of the mitochondrial tyrosyl-tRNA synthetase gene, YARS2, causes myopathy, lactic acidosis, and sideroblastic anemia-MLASA syndrome. Am J Hum Genet, 2010. 87(1): p. 52-9.

41. Pierce, S.B., et al., Mutations in LARS2, encoding mitochondrial leucyltRNA synthetase, lead to premature ovarian failure and hearing loss in Perrault syndrome. Am J Hum Genet, 2013. 92(4): p. 614-20.

42. Simon, M., et al., Mutations of human NARS2, encoding the mitochondrial asparaginyl-tRNA synthetase, cause nonsyndromic deafness and Leigh syndrome. PLoS Genet, 2015. 11(3): p. e1005097.

43. Kopajtich, R., et al., Biallelic IARS Mutations Cause Growth Retardation with Prenatal Onset, Intellectual Disability, Muscular Hypotonia, and Infantile Hepatopathy. Am J Hum Genet, 2016. 99(2): p. 414-22. 
Chapter 5

Supplementary data due to many presented columns in Excel processed in download link: https://www.nature.com/articles/s41431-017-0058-2

Table 4 List of variants after filtering Patient I.2.2

Table 5 List of variants after filtering Patient II.2.1

Table 6 List of variants after filtering Patient III.2.1 


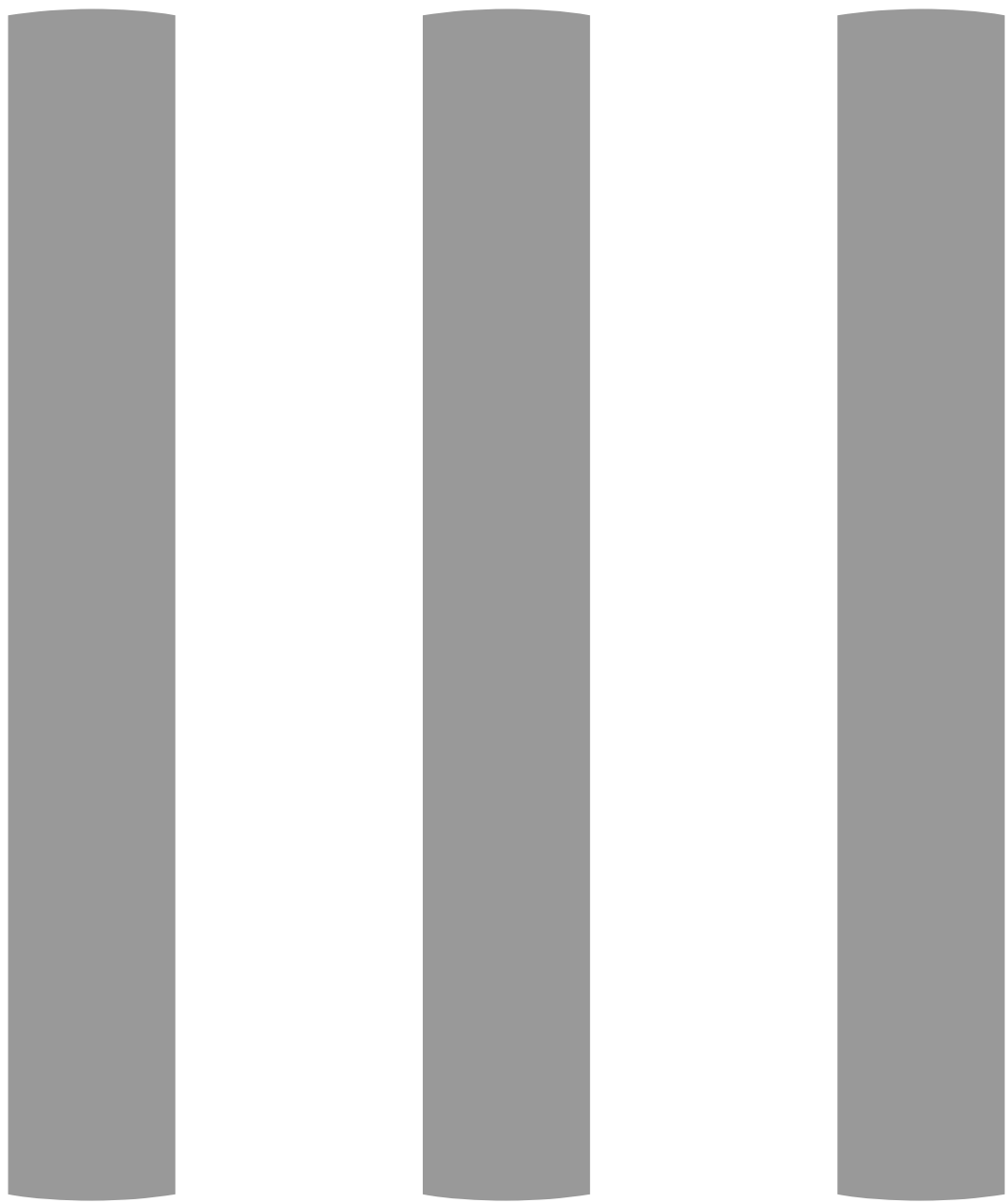
Part III

Characterization of Mitochondrial

Bottleneck 
CHAPTER 6 


\section{Tfam Depletion Leads to a Strong Reduction in mtDNA Copy Number OXPHOS Deficiency and Developmental Abnormalities in Zebrafish Embryos}

Auke BC Otten\#, Rick Kamps\#, Patrick Lindsey, Mike Gerards, Hélène Pendeville-Samain, Marc Muller, Florence HJ van Tienen*, Hubert JM Smeets*

\# Joint first author, equal contribution

*Joint last author, equal contribution 


\section{Abstract}

High mitochondrial DNA (mtDNA) copy numbers are essential for oogenesis and embryogenesis and correlate with fertility of oocytes and viability of embryos. To understand the pathology and mechanisms associated with low mtDNA copy numbers, we knocked down mitochondrial transcription factor A (tfam), a regulator of mtDNA replication, during early zebrafish development. Reduction of tfam using a splice-modifying morpholino (MO) resulted in a $42 \% \pm 17 \%$ decrease in mtDNA copy number in embryos at 4 days post fertilization. Morphant embryos displayed abnormal development of the eye, brain, heart and muscle, as well as a $50 \% \pm 22 \%$ decrease in ATP production. Transcriptome analysis revealed a decrease in protein-encoding transcripts from the heavy strand of the mtDNA, and down-regulation of genes involved in haem production and the metabolism of metabolites, which appear to trigger increased rRNA and tRNA synthesis in the nucleoli. However, this stress or compensatory response appears to fall short as pathology emerges and expression of genes related to eye development are severely down-regulated. Taken together, this study highlights the importance of sufficient mtDNA copies for early zebrafish development. Zebrafish is an excellent model to manipulate the mtDNA bottleneck and study its effect on embryogenesis rapidly and in large numbers of offspring. 


\section{Introduction}

Mitochondria are responsible for producing the majority of cellular energy in the form of ATP. Depending on the tissue type and energy requirement, a cell can contain up to thousands of mitochondria, each having multiple copies of mitochondrial DNA (mtDNA). Of all cell-types, mtDNA copy numbers are the highest in oocytes, ranging between 100,000 and 400,000 copies in mammals, such as rodents, cows and humans, and up to 16,5 million copies in zebrafish [1]. The high mtDNA copy number in oocytes is established by an initial reduction during embryogenesis, called the mitochondrial bottleneck, followed by clonal expansion of the mtDNA during oogenesis appears to be important for successful fertilization and embryogenesis [2]. In mice, the oocyte mtDNA copy number should be sufficient for normal development until implantation at day 4 , and it has been demonstrated that oocytes with less than 50,000 mtDNA copies fail to resume development after implantation [3,4]. This negative correlation between mtDNA copy number and developmental competence of embryos has also been suggested for human oocytes [5].

Mitochondrial transcription factor A (TFAM), a protein of the high mobility group box-family, is a key activator of mtDNA replication and transcription [6] and is crucial for the regulation of mtDNA copy number [7]. A direct relation between the mtDNA copy number and the total TFAM protein level has been demonstrated in mice embryos with a heterozygous or homozygous disruption of the Tfam gene [8]. Homozygous TFAM knockout (KO) mouse embryos displayed a strong mtDNA depletion with severely reduced functioning of the electron transport chain (ETC) and died after gastrulation and implantation, while heterozygous KO TFAM mice had moderately reduced mtDNA levels and respiratory chain deficiency, which was strongest in the developing heart [7]. These studies demonstrated the importance of a sufficient mtDNA copy number during oogenesis and embryogenesis, but the mechanism by which a reduced mtDNA copy number affects embryogenesis is currently unknown.

Studying embryonic development in zebrafish overcomes ethical and practical obstacles associated with the use of human or mouse embryos. Zebrafish are vertebrates, $>70 \%$ of human genes have at least one zebrafish orthologue, and 
the major tissues and organs are the same. Zebrafish are transparent during early development and have a high number of offspring. Organs develop within 5 days post fertilization and gene knockdown during early embryogenesis can be performed by injection of morpholino antisense oligonucleotides (MOs) [9]. Previously, we showed that the mitochondrial bottleneck during early development is highly conserved between zebrafish and mammals [10]. In addition, the zebrafish Tfam protein is functionally homologous to its human counterpart and is expressed ubiquitously from the earliest stages of development [11]. In this study, we performed tfam knockdown during zebrafish embryogenesis in order to reduce the mtDNA copy number during early development. In this way, we created an animal model with a tuneable mtDNA bottleneck, which allows us to assess the effect of differences in mtDNA copy number on OXPHOS function and embryonic development and to determine the underlying mechanisms.

\section{Material and Methods}

\section{Zebrafish Maintenance and Procedures}

Zebrafish (Danio rerio) were housed and raised in the zebrafish facility of the University of Liège as described before [12]. To retrieve eggs, wild-type adult male and female zebrafish were separated within the same breeding tank by a plastic divider the day before breeding. This separation was removed the next day after the light was turned on in order to allow natural mating and eggs were collected after spawning. Eggs were collected in Petri dishes containing 1x E3 medium for zebrafish at $28^{\circ} \mathrm{C}\left(580 \mathrm{mg} / \mathrm{l} \mathrm{NaCl}, 27 \mathrm{mg} / \mathrm{l} \mathrm{KCl}, 97 \mathrm{mg} / \mathrm{l} \mathrm{CaCl} 2 \cdot 2 \mathrm{H}_{2} \mathrm{O}\right.$, $163 \mathrm{mg} / \mathrm{l} \mathrm{MgCl} \cdot 6 \mathrm{H}_{2} \mathrm{O}$ and $0.0001 \%$ methylene blue (Sigma-Aldrich), pH 7.2) [12]. Embryos were microscopically staged according to the embryonic development as described before [13]. Unless stated otherwise, all reagents used in this study were obtained from Thermo Fisher Scientific.

\section{Tfam Knockdown Experiments}

Antisense morpholino oligonucleotides (MO) were purchased from Gene Tools and micro-injected into one or two-cell stage embryos. A splice modifying MO was used, targeting the boundary of exon 2 and intron 2-3 of the zebrafish tfam gene 
(Ensemble: ENSDART00000092009, tfam splice-MO: 5'CGGCAGATGGAAATTTACCAGGATT-3'). For controlling the effect of the MO injection, an equal concentration of a standard control-morpholino (Ctrl-MO: 5'CСTCTTACCTCAGTTACAATTTATA-3') was used in separate embryos of the same batch during each experiment. All MOs were dissolved in $1 \times$ Danieau buffer (58 $\mathrm{mM} \mathrm{NaCl}, 0.7 \mathrm{mM} \mathrm{KCl}, 0.4 \mathrm{mM} \mathrm{MgSO} 4,0.6 \mathrm{mM} \mathrm{Ca}\left(\mathrm{NO}_{3}\right)_{2}$, and $5.0 \mathrm{mM} \mathrm{HEPES} \mathrm{pH}$ 7.6) and $0.5 \%$ rhodamine dextran (Thermo Fisher) was added to the MO solution upon injection. At $1 \mathrm{hpf}, 1 \mathrm{nl}$ was injected into each embryo using microinjection [14]. The next day, distribution of the rhodamine dextran dye was checked using fluorescence stereomicroscopy to verify proper injections. Only those embryos in which the rhodamine dextran dye was visible were used for follow-up investigations.

\section{Quantitative and Qualitative Analysis of Tfam RNA}

Total RNA of 2 - 4 dpf zebrafish embryos was extracted using Trizol reagent and purified using the RNeasy Mini Kit (Qiagen), according to manufacturer's instructions. cDNA synthesis was performed with $500 \mathrm{ng}$ RNA in the presence of first strand buffer, $0.75 \mu \mathrm{g}$ oligo-dT, $0.75 \mu \mathrm{g}$ random hexamer-primer, $50 \mathrm{nmol}$ dNTPs (GE Healthcare Life Sciences), 1 U RNAse inhibitor (RNAsin, Promega) and $5 \mathrm{U}$ reverse transcriptase for $60^{\prime}$ at $42^{\circ} \mathrm{C}$, followed by $5^{\prime}$ at $95^{\circ} \mathrm{C}$. The effect on tfam splicing was assessed using RT-PCR amplification of $25 \mathrm{ng}$ cDNA in a PCR mix contained under standard conditions, using $0.6 \mu \mathrm{M}$ forward primer, $0.6 \mu \mathrm{M}$ reverse primer (Supplementary Table S1). PCR conditions were $5^{\prime}$ denaturation at $94^{\circ} \mathrm{C}, 35$ cycles of $1^{\prime}$ at $94^{\circ} \mathrm{C}, 1^{\prime}$ at $58^{\circ} \mathrm{C}$ and $1,5^{\prime}$ at $72^{\circ} \mathrm{C}$, followed by $7^{\prime}$ at $72^{\circ} \mathrm{C}$. The PCR product was sequenced by conventional Sanger sequencing. tfam gene expression was quantified by comparing the RNA expression ratio of Tfam RNA to $18 S$ RNA. Per reaction, $2.5 \mathrm{ng}$ cDNA was amplified in a $10 \mu \mathrm{l}$ reaction containing 1x Sensimix Sybr Hi-Rox reagents (Bioline) and $375 \mathrm{nM}$ of both forward and reverse primer (Supplementary Table S1). Real-time PCR was performed on an ABI7900HT using the following settings: $10^{\prime} 95^{\circ} \mathrm{C}, 40$ cycles of $15^{\prime \prime} 95^{\circ} \mathrm{C}$ and $1^{\prime}$ $60^{\circ} \mathrm{C}$. The statistical analysis was performed by a Student's t-test. $p$-values $<0.05$ were considered significant. 


\section{Quantification of mtDNA Copy Number}

To determine mtDNA copy number, embryos were individually collected in sterile tubes and directly frozen without water. For DNA isolation, embryos were thawed and lysed for 4 hours at $55^{\circ} \mathrm{C}$ in lysis buffer containing $75 \mathrm{mM} \mathrm{NaCl}, 50 \mathrm{mM}$ EDTA, $20 \mathrm{mM}$ HEPES, $0.4 \%$ SDS and $200 \mu \mathrm{g}$ proteinase $\mathrm{K}$ (Sigma) while vortexing repeatedly. DNA precipitation was performed overnight at $-20^{\circ} \mathrm{C}$ after adding 420 $\mu \mathrm{l}$ isopropanol. Following centrifugation, the DNA pellet was washed with $70 \%$ ethanol and dissolved in Tris-EDTA buffer. The relative mtDNA abundance was quantified by measuring the steady-state amount of mitochondrial ND1 and nuclear B2M. Per reaction, $20 \mathrm{ng}$ DNA was used in a $10 \mu \mathrm{l}$ reaction containing $1 \mathrm{x}$ Sensimix Sybr Hi-Rox reagents (Bioline) and $375 \mathrm{nM}$ of both forward and reverse primer (Supplementary Table S1). Real-time quantification was performed on an $\mathrm{ABI7900HT}$ as described before. Statistical analysis was carried out using oneway analysis of variance (ANOVA) followed by the Bonferroni multiple comparisons test. $P$-values $<0.05$ were considered significant.

\section{Imaging Zebrafish Embryos}

Zebrafish embryos were fixed at $4^{\circ} \mathrm{C}$ with paraformaldehyde for 4 hours and standard paraffin serial sections of $4 \mu \mathrm{m}$ thickness were prepared for immunostaining and hematoxylin-eosin (HE)-staining. Immunohistochemistry was performed following a microwave heat-induced antigen retrieval step for four times $5^{\prime}$ at 650 Watt (in Tris-EDTA, pH=9.0) and was analysed with the Dako REALTM EnVisionTM Detection System, Peroxidase/DAB, Rabbit/Mouse, using a Dako automated immunostaining instrument and protocols according to the manufacturer (Agilent, Santa Clara). Antibodies and conditions used are listed in Supplementary Table S1. HE-staining was performed to reveal the underlying embryonic structures. Paraffin slides were embedded in Entellan (Merck) and protected by cover slips (Knittel Glass). Microscopic images were taken by using the Nikon Eclipse E80 Imaging System (Nikon) at different magnifications. The orientation of fish was determined according to the Zebrafish Bio-Atlas [15]. 


\section{Oxygen Consumption Rate}

At $4 \mathrm{dpf}$, chorion-free living fish embryos with a heartbeat and active swimming behaviour upon touching were selected and collected individually. The oxygen consumption rate (OCR) (pmol/minute) was measured every minute for 15 minutes in a Seahorse XF24 system (Agilent/Seahorse Biosciences), according to the islet protocol of the manufacturer with 2 fish per well of a 24-well plate. Five minutes after starting the assay, $80 \mu \mathrm{M}$ of Oligomycin (Sigma-Aldrich) was added and $60 \mu \mathrm{M}$ for Antimycin \& Rotenone (Sigma-Aldrich) in the injecting assay medium (1x E3 medium) was added 10 minutes after start. The OCR level at basal respiration ( $\mathrm{pmol} / \mathrm{min}$ ) was calculated using the OCR values at the start respiration level (time-point 5 minutes) minus antimycin/rotenone (AMA) level at time-point 10 minutes. Maximal ATP production ( $\mathrm{pmol} / \mathrm{min}$ ) was determined as start respiration level minus oligomycin (OMY) level at time-point 15 minutes. The statistical analysis was performed by a Student's t-test. $p$-values $<0.05$ were considered significant.

\section{Gene Expression and Pathway Analysis}

For gene expression studies, tfam splice-MO and control-MO injected embryos and non-injected control embryos at $4 \mathrm{dpf}$, were individually collected in sterile tubes. Selection criteria were the same as for the OCR measurements. RNA from single embryos was isolated using the MagMax-96 for microarray kit (Ambion) and 200 ng RNA was labelled (Cyanine 3-CTP and Cyanine 5-CTP), fragmented and hybridized using the Low Input Quick Amp Labeling Kit, Two-colour (Ambion), according to manufacturer's instructions. After labelling, amplified cRNA samples were purified using the RNeasy Mini kit (Qiagen) and Cyanine 3 and Cyanine 5 dye concentration, RNA absorbance ratio (260/280nm) and cRNA concentration were quantified using the nanodrop $2000 \mathrm{C}$ (Thermoscientific). Only samples with a yield $>0.825 \mu \mathrm{g}$ and a specific dye activity $>6.0 \mathrm{pmol} / \mathrm{\mu g}$ were used for fragmentation and hybridization. For fragmentation, $825 \mathrm{ng}$ labelled CRNA was used and the final volume was adjusted to $41.8 \mu \mathrm{l}$ with RNAse-free water, followed by the hybridization procedure, as described by the manufacturer (Ambion). Dyeswap hybridizations $(2+2)$ were performed on microarray slides $(4 \times 44 \mathrm{~K}$ zebrafish V3, Agilent) using gasket slides and a hybridization chamber and incubated for 17 $\mathrm{h}$ at $65^{\circ} \mathrm{C}$ and $10 \mathrm{rpm}$ in the hybridization oven (Agilent Technologies). Slides 
were washed with Triton X-102, freshly added to the Wash Buffers. Microarray slides were scanned using a DNA Microarray scanner with Surescan HighResolution Technology (Model 2565CA, Agilent). All microarray data is deposited in the GEO database, accession GSE146696.

The arrays contained 45,220 probes. Each probe identifier was transformed to Ensembl gene IDs (ENSDARG). This resulted in 36,156 probes containing a nonempty transcript ID of which 19,459 were unique transcripts and kept for the analysis. All transcripts were analysed using a multivariate Gaussian linear regression ( $\operatorname{MVN}(\mu, \Sigma)$ where $\mu$ is the mean, $\sum$ is the covariance matrix

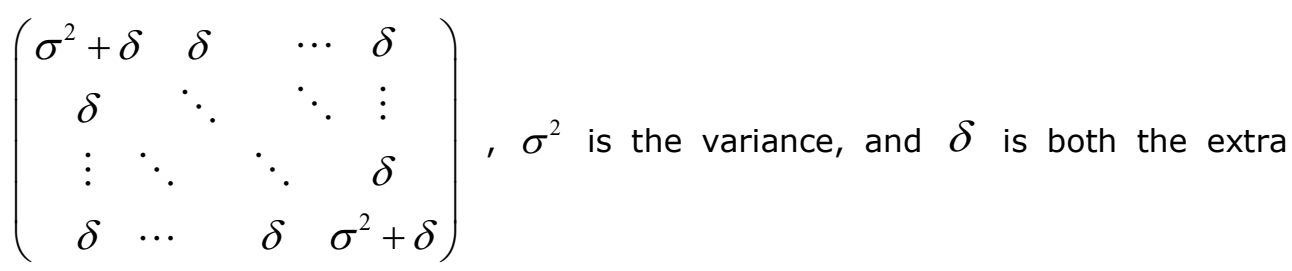
component of variance across subjects and the common covariance among responses on the same subject) including slide differences (Slide), dye swap (Dye), background level (Bg), injection (Inj) and a random effect. The inference criterion used for comparing the models is their ability to predict the observed data, i.e. models are compared directly through their minimized minus loglikelihood. When the numbers of parameters in models differ, they are penalized by adding the number of estimated parameters, a form of the Akaike information criterion [16]. For each transcript, a model containing the relevant covariates mentioned above $(E(y)=$ Slide+Dye+Bg+Inj) was fitted in order to obtain a reference AIC. Then a model containing the treatment group (Trt) was fitted $(E(y)=$ Slide+Dye+Bg+Inj+Inj:Trt). The transcript under consideration was found to be differentially expressed if the AIC of this second model decreased compared to the model not containing the treatment. These statistical analysis were performed using the freely available program $\mathrm{R}$ [17] and the publicly available libraries 'rmutil' and 'growth' [18]. An unbiased Gene-ontology analysis and visualization of microarray data on biological pathways was performed using PANTHER (Protein Analysis THrough Evolutionary Relationships) with the Danio rerio (Dr_Derby_Ensembl_80) gene product/protein database [19, 20]. 
Differentially expressed genes (DEGs) were mapped to unique Entrez Gene IDs and to Gene Ontology (GO) classes, using the PANTHER Overrepresentation Test (Released 2018-02-03) enrichment method of PANTHER version 13.1.

\section{Results}

Tfam Knockdown Leads to Reduced mtDNA Content and Reduced Mitochondrial Function

To establish decreased mtDNA copy number, we microinjected an antisense splice-MO targeting tfam-mRNA in zebrafish embryos. Using RT-PCR and Sanger sequencing of cDNA from tfam splice-MO-injected $4 \mathrm{dpf}$ embryos ( $n=6 /$ group), we showed that the tfam splice-MO causes a 128 base-pair deletion of exon 2 (c.84_211del), predicted to cause a frameshift and a premature stop codon (p.(Cys29Hisfs*36)) (Figure 1A, Supplementary Figure S1). Quantitative PCR analysis of tfam exon 5 and exon $6-7$ showed respectively $59 \% \pm 2 \%$ and $60 \% \pm 5 \%$ decrease in expression in tfam splice-MO injected embryos at day 4 (mean $\pm S D$, $\mathrm{n}=6$ /group). qPCR analysis of tfam exon 2 expression showed a reduction of $80 \pm 3 \%$ at day 4 in zebrafish ( $n=6 /$ group) injected with 2 ng tfam splice-MO compared to the Ctrl-MO (Supplementary Figure S2). This indicates that $\sim 60 \%$ of tfam RNA is subjected to nonsense-mediated decay and that of the residual $\sim 40 \%$ tfam RNA, only half is exon 2-containing wild-type RNA (only $20 \%$ of normal tfam amount). Next, we assessed the mtDNA copy number in non-injected, Ctrl-MO injected and tfam splice-MO injected embryos ( $n=20$ in each 2 ng group, and $n=$ 16 in each 4 ng group). At 4 dpf, a significant, 42\% $\pm 17 \%$ (mean $\pm S D$ ) decrease in the mtDNA copy number was observed in embryos injected with either $2 \mathrm{ng}$ or 4 ng tfam splice-MO (Figure 1B). In contrast, no significant differences in mtDNA copy number were apparent between groups at $2 \mathrm{dpf}$ (Supplementary Figure S3). To assess if decreased mtDNA copy number also affected mitochondrial capacity, we measured the oxygen consumption rate (OCR) at $4 \mathrm{dpf}$. As shown in Figure 1C, both the OCR of the basal respiration and the ATP production were significantly decreased in the 2 ng tfam splice-MO treated zebrafish $(n=10)$ compared with the $2 \mathrm{ng}$ control-MO treated group $(n=9)(p<0.05)$ by respectively $40 \% \pm 25 \%$ and $50 \% \pm 22 \%$ (mean \pm SD). 
A

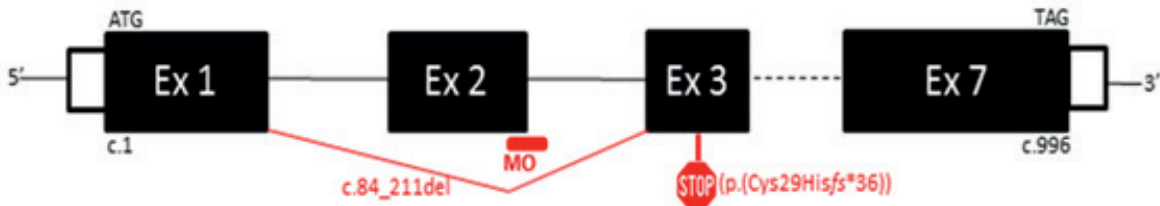

B

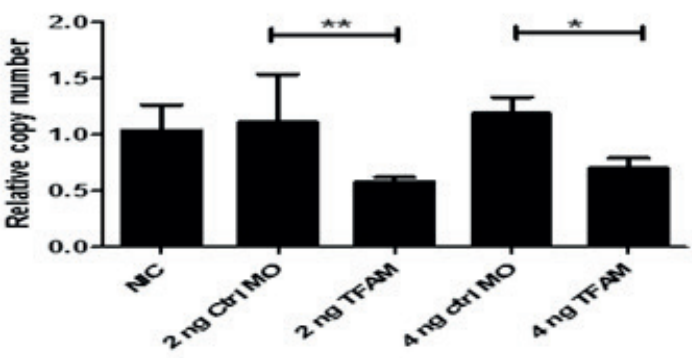

C
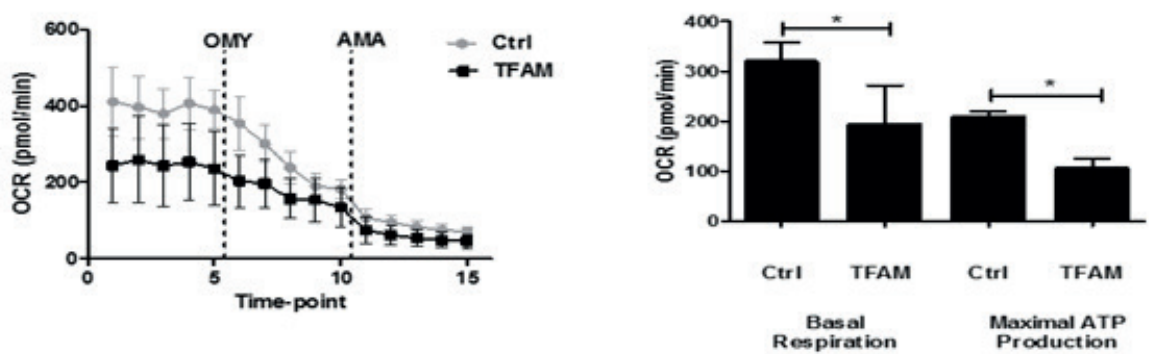

Figure 1. Knockdown of TFAM in zebrafish embryos. Zebrafish embryos injected with indicated amount of either Control-morpholino (Ctrl-MO) or tfam splice-morpholino (TFAM-MO) at $1 \mathrm{hpf}$ and analysed at $4 \mathrm{dpf}$. (A) tfam splice-morpholino (MO) at the 3' splice site of exon 2 causes deletion of exon 2, which predicts a frame-shift and premature stop codon (p.(Cys29Hisfs*36)) (figure is not on scale). (B) The relative copy number assessed by mitochondrial ND1 / nuclear B2M ratio. Data are normalized to the NIC embryos $(n=7)$. Bars indicate mean values with $S D, n=20$ per 2 ng injected condition and $n=16$ per $4 \mathrm{ng}$ injected condition. $P$-values are calculated using ANOVA followed by Bonferroni's Multiple Comparison Test to assess copy number, $* P$-value $<0.05, * * P$ value $<0.01$. (C) The oxygen consumption rate (OCR) measured by Seahorse XF24 in order to assess basal respiration and maximal ATP production capacity at $4 \mathrm{dpf}$ using $2 \mathrm{ng}$ MO. The statistical analysis was performed by using a student t-test $(P<0.05), \mathrm{n}=10$ per tfam condition and $\mathrm{n}=9$ per ctrl condition. 
Tfam Knockdown Causes Brain, Eye, Heart, and Muscle Abnormalities

At 4 dpf, morphant embryos displayed morphological abnormalities compared to control embryos. The macroscopic phenotype included overall oedema, curved tails, necrotized yolk sacs and small eyes (Supplementary Figure S4). Fish injected with $4 \mathrm{ng}$ tfam splice-MO were more severely affected than $2 \mathrm{ng}$ tfam splice-MO ( $n=100$ injected per condition), as they had a higher count for oedema, curved tails, necrotized yolk sac, small eyes, and developmental delay. The percentage of dead embryos was $<1 \%$ when injecting 2 or $4 \mathrm{ng}$ of tfam $\mathrm{MO}$-injections at 4 dpf (Supplementary Figure S4). In contrast, injection of $6 \mathrm{ng}$ of tfam splice-MO showed $>70 \%$ of embryonic lethality due to severe developmental complications. Macroscopic inspection of tfam splice-MO injected embryos showed that all observed pathologies manifested at 3 or $4 \mathrm{dpf}$, whereas development was apparently normal at $2 \mathrm{dpf}$. These morphological changes parallel the decrease in mtDNA copy number that is observed at $4 \mathrm{dpf}$, but not at $2 \mathrm{dpf}$ (Figure 1B, Supplementary Figure S3). Since the decrease in mtDNA content was comparable with $4 \mathrm{ng}$ tfam splice-MO, the dosage of $2 \mathrm{ng}$ tfam splice-MO was used in all following experiments, reducing the risk of non-specific observations.

Microscopic evaluation of zebrafish ( $n=13$ per condition) with reduced mtDNA copy number using 2ng tfam splice-MO showed impaired development compared to control embryos (Figure 2). Brain size was decreased with no or less welldeveloped cerebellum (Figure 2B) and eyes smaller and the different layers lesswell organized compared to controls (Figure 2D). Also, the organization of the myotomic area was less compact with the skeletal muscle fibres being thinner and disorganized (Figure 2F). Finally, morphant fish displayed marked pericardial oedema, alongside with a dilated non-looped heart (Figure $\mathbf{2 H}$ ). 

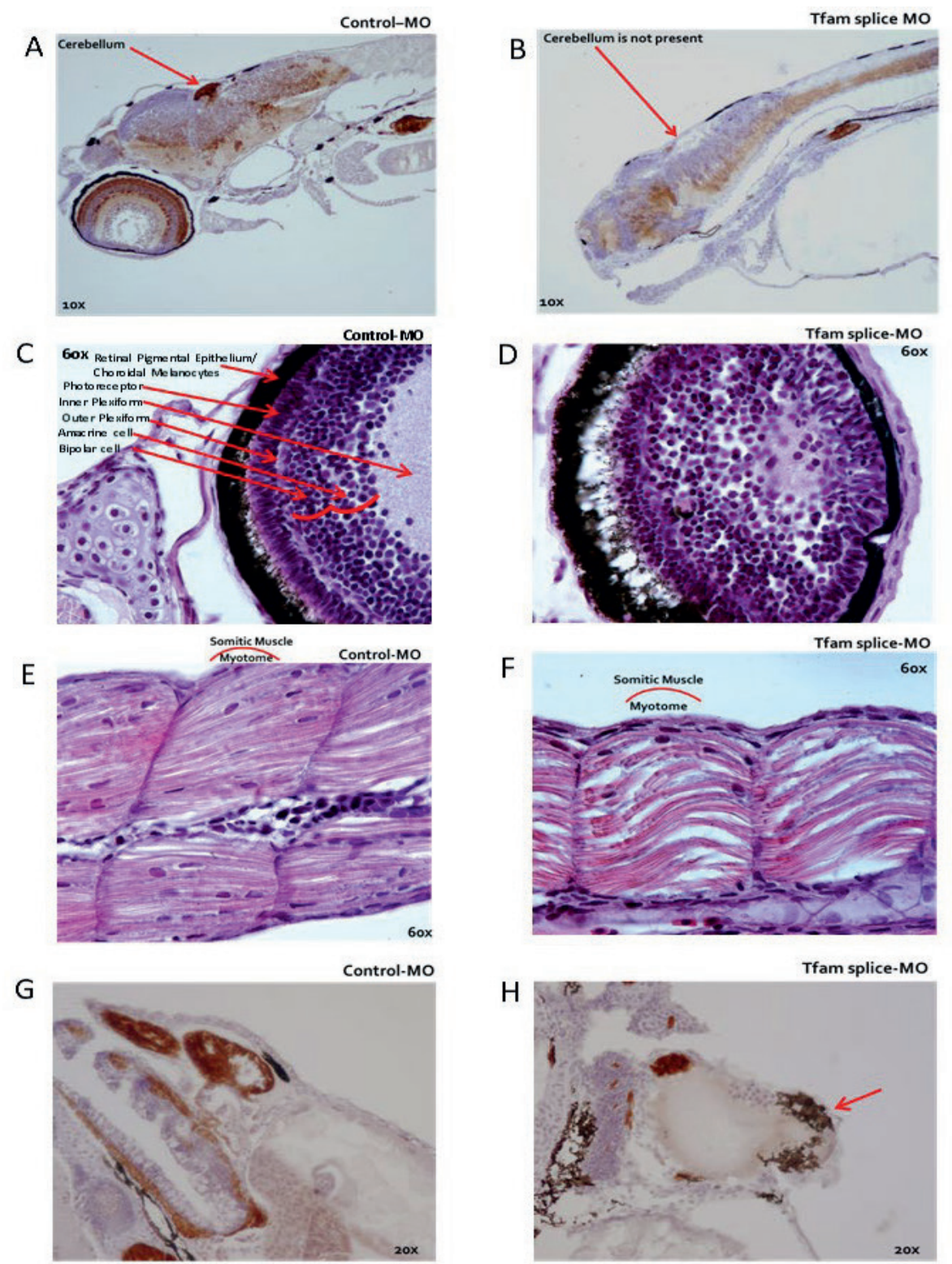

Figure 2. Microscopy of zebrafish embryos following TFAM knockdown. Image $(A-H)$ : Microscopic analysis following serial HE-staining of zebrafish embryos at $4 \mathrm{dpf}$, which were injected at $1 \mathrm{hpf}$ with either $2 \mathrm{ng}$ Tfam splice-MO or $2 \mathrm{ng}$ Ctrl-MO, $\mathrm{n}=13$ each condition. (B) The cerebellum was missing in the phenotype or a delayed development of this cerebellum in these fish. (D) The eyes were smaller and the eye layers were less developed. (F) A muscle phenotype was clearly visible in the dorsal fin, especially in the myotomic area, which was less compact in these somatic muscles. $(\mathrm{H})$ The fish displayed a marked pericardial oedema (red arrow). They did not develop a normally looped heart as in Ctrl-MO (G), but the hearts were dilated in the phenotypes $(H)$. 
Genes and Pathways Altered by Tfam Knockdown

To characterize the underlying pathogenic processes due to tfam knockdown and subsequent decrease in mtDNA copy number, we compared global gene expression profiles at $4 \mathrm{dpf}$ of zebrafish injected with $2 \mathrm{ng}$ of either tfam spliceMO $(n=12)$ or Ctrl-MO $(n=12)$. A group of non-injected control zebrafish was also included $(n=9)$ to control for the injection effect in the microarray analysis and allow identification of transcripts altered in the tfam splice-MO zebrafish compared with the Ctrl-MO zebrafish. A total of 19,459 transcripts were present on the array (Supplementary Table S2), of which 16,631 (85.5\%) had a signal intensity higher than twice the background signal in both conditions or a signal that was at least three times higher than the background signal in either the group of tfam splice-MO or Ctrl-MO zebrafish. Of these, 3,158 transcripts (19.3\%) were differentially expressed between the two groups with a fold change (FC) $>50 \%$. Expression of 2,063 transcripts $(64.3 \%$ ) was increased (FC $\geq 1.50$ ), while expression of 1,145 transcripts $(35.7 \%)$ was decreased in the tfam splice-MO injected embryos ( $F C \leq 0.67)$ ). In order to identify significantly altered pathways by using gene ontology analysis, Protein ANalysis THrough Evolutionary Relationships (PANTHER) was used. The microarray contained 19,459 transcripts, of which 17,395 could be annotated (89.4\%) and were included in the PANTHER analysis. A total of 18 significantly enriched GO biological processes (FDR <0.05) were identified and these were manually grouped into five groups including the underlying GO terms, namely: tRNA processing, ribosome biogenesis, RNA surveillance and catabolism, drug metabolism, and visual perception (Table 1, Supplementary Table S4). As the RNA-related processes differ for nuclear and mtDNA genes and for that reason should not be considered as one group, we also listed the mitochondria-related processes, characterized by mitochondria-related GO terms (Table 2, Supplementary Table S5). Most of the mitochondria related GO terms were small, comprising less than 50 measured genes, but sufficient number of genes were interrogated to conclude that these processes were not significantly enriched by the mtDNA reduction. However, as expression of mtDNA encoded proteins, which is regulated by TFAM, was not covered in a GO term, we checked the expression of the 8 mtDNA protein encoding genes manually. The 8 
mtDNA proteins encoding transcripts of the $\mathrm{H}$-strand were significantly decreased, whereas the single L-strand transcript was unaltered (Supplementary Table S3). The remainder four protein encoding transcripts of the $\mathrm{H}$-strand were not present on the array (atp6, atp8, cox 3, and cytb).

Table 1 . Significantly altered GO biological process terms.

\begin{tabular}{|c|c|c|c|c|}
\hline Description & $\begin{array}{l}\text { Measured } \\
\quad(\mathbf{n})\end{array}$ & Changed (n) & $\begin{array}{c}\text { Fold } \\
\text { Enrich } \\
\text { ment }\end{array}$ & FDR \\
\hline \multicolumn{5}{|l|}{ tRNA processing } \\
\hline GO:0006396 RNA processing & 426 & $\begin{array}{c}106 \\
(\mathrm{Cm}=15, \mathrm{Cn}=91)\end{array}$ & 1.52 & $2.4 \mathrm{E}-02$ \\
\hline $\begin{array}{l}\rightarrow \text { GO:0034660 ncRNA metabolic } \\
\text { process }\end{array}$ & 260 & $\begin{array}{c}88 \\
(\mathrm{Cm}=30, \mathrm{Cn}=58)\end{array}$ & 2.06 & $5.2 \mathrm{E}-06$ \\
\hline $\begin{array}{l}\rightarrow \mathrm{GO}: 0006399 \text { tRNA metabolic } \\
\text { process }\end{array}$ & 118 & $\begin{array}{c}44 \\
(\mathrm{Cm}=22, \mathrm{Cn}=22)\end{array}$ & 2.27 & $1.4 \mathrm{E}-03$ \\
\hline $\begin{array}{l}\rightarrow \text { GO:0009451RNA } \\
\text { modification }\end{array}$ & 88 & $\begin{array}{c}32 \\
(\mathrm{Cm}=9, \mathrm{Cn}=23)\end{array}$ & 2.21 & $2.6 \mathrm{E}-04$ \\
\hline$\rightarrow$ GO:0034470 ncRNA processing & 197 & $\begin{array}{c}72 \\
(\mathrm{Cm}=14, \mathrm{Cn}=58)\end{array}$ & 2.22 & 4.3E-06 \\
\hline$\rightarrow$ GO:0008033tRNA processing & 73 & $\begin{array}{c}27 \\
(\mathrm{Cm}=7, \mathrm{Cn}=20)\end{array}$ & 2.25 & $3.8 \mathrm{E}-02$ \\
\hline \multicolumn{5}{|l|}{ Ribosome biogenesis } \\
\hline $\begin{array}{l}\text { GO:0022613 Ribonucleoprotein } \\
\text { complex biogenesis }\end{array}$ & 239 & $\begin{array}{c}69 \\
(\mathrm{Cm}=14, \mathrm{Cn}=55)\end{array}$ & 1.76 & $1.0 \mathrm{E}-02$ \\
\hline$\rightarrow$ GO:0042254 Ribosome biogenesis & 162 & $\begin{array}{c}60 \\
(\mathrm{Cm}=14, \mathrm{Cn}=46)\end{array}$ & 2.25 & 4.3E-05 \\
\hline$\rightarrow$ GO:0006364 rRNA processing & 105 & $43(\mathrm{Cm}=6, \mathrm{Cn}=37)$ & 2.49 & $2.0 \mathrm{E}-04$ \\
\hline \multicolumn{5}{|l|}{ RNA surveillance and catabolism } \\
\hline GO:0071025 RNA surveillance & 9 & $9(\mathrm{Cm}=0, \mathrm{Cn}=9)$ & 6.09 & $2.3 \mathrm{E}-02$ \\
\hline $\begin{array}{l}\rightarrow \text { GO:0071027 Nuclear RNA } \\
\text { surveillance }\end{array}$ & 8 & $8(\mathrm{Cm}=0, \mathrm{Cn}=8)$ & 6.09 & $3.4 \mathrm{E}-02$ \\
\hline $\begin{array}{l}\quad \rightarrow \text { GO:0071028 Nuclear mRNA } \\
\text { surveillance }\end{array}$ & 8 & $8(\mathrm{Cm}=0, \mathrm{Cn}=8)$ & 6.09 & $3.6 \mathrm{E}-02$ \\
\hline
\end{tabular}




\begin{tabular}{|c|c|c|c|c|}
\hline Description & $\begin{array}{l}\text { Measured } \\
\text { (n) }\end{array}$ & Changed (n) & $\begin{array}{c}\text { Fold } \\
\text { Enrich } \\
\text { ment }\end{array}$ & FDR \\
\hline $\begin{array}{l}\qquad \rightarrow \mathrm{GO}: 0034475 \text { U4 snRNA 3'- } \\
\text { end processing }\end{array}$ & 8 & $8(\mathrm{Cm}=0, \mathrm{Cn}=8)$ & 6.09 & $3.2 \mathrm{E}-02$ \\
\hline $\begin{array}{l}\rightarrow \text { GO:0016075 rRNA catabolic } \\
\text { process }\end{array}$ & 11 & $9(\mathrm{Cm}=0, \mathrm{Cn}=9)$ & 4.98 & $3.6 \mathrm{E}-02$ \\
\hline \multicolumn{5}{|l|}{ Drug metabolism } \\
\hline GO:0042737 Drug catabolic process & 85 & $\begin{array}{c}33 \\
(\mathrm{Cm}=2, \mathrm{Cn}=31)\end{array}$ & 2.36 & $1.1 \mathrm{E}-02$ \\
\hline \multicolumn{5}{|l|}{ Visual perception } \\
\hline GO:0007601 Visual perception & 83 & $\begin{array}{c}31 \\
(\mathrm{Cm}=2, \mathrm{Cn}=29)\end{array}$ & 2.27 & $2.3 \mathrm{E}-02$ \\
\hline $\begin{array}{l}\rightarrow \mathrm{GO}: 0050953 \text { Sensory perception } \\
\text { of light stimulus }\end{array}$ & 88 & $\begin{array}{c}31 \\
(\mathrm{Cm}=2, \mathrm{Cn}=29)\end{array}$ & 2.14 & $3.5 \mathrm{E}-02$ \\
\hline
\end{tabular}

GO terms biological process with FDR $<0.05$ were considered to be significantly changed. $\mathrm{Cm}$ are changed genes that are linked to mitochondria; $\mathrm{Cn}$ are changed genes linked to the nucleus; FDR is False Discovery Rate. 
Table 2. Mitochondria-related GO biological processes

\begin{tabular}{|c|c|c|c|}
\hline Description & $\begin{array}{l}\text { Measured } \\
\text { (n) }\end{array}$ & $\begin{array}{c}\text { Changed } \\
\text { (n) }\end{array}$ & $\begin{array}{c}\text { Fold } \\
\text { Enrichment }\end{array}$ \\
\hline GO:0007005 mitochondrion organization & 152 & 15 & 0.60 \\
\hline $\begin{array}{l}\text { GO:0007007 inner mitochondrial membrane } \\
\text { organization }\end{array}$ & 20 & 2 & 0.61 \\
\hline $\begin{array}{l}\text { GO:0007008 outer mitochondrial membrane } \\
\text { organization }\end{array}$ & 1 & 0 & \\
\hline GO:0006390 mitochondrial transcription & 8 & 2 & 1.52 \\
\hline GO:0032543 mitochondrial translation & 27 & 8 & 1.80 \\
\hline GO:0006626 protein targeting to mitochondrion & 32 & 1 & 0.19 \\
\hline $\begin{array}{l}\text { GO:0070585 protein localization to } \\
\text { mitochondrion }\end{array}$ & 32 & 1 & 0.19 \\
\hline $\begin{array}{l}\text { GO:0042775 mitochondrial ATP synthesis } \\
\text { coupled electron transport }\end{array}$ & 41 & 7 & 1.04 \\
\hline GO:0006119 oxidative phosphorylation & 46 & 9 & 1.19 \\
\hline $\begin{array}{l}\text { GO:0033108 mitochondrial respiratory chain } \\
\text { complex assembly }\end{array}$ & 25 & 2 & 0.49 \\
\hline $\begin{array}{l}\text { GO:0015986 ATP synthesis coupled proton } \\
\text { transport }\end{array}$ & 20 & 1 & 0.30 \\
\hline GO:0006979 response to oxidative stress & 70 & 9 & 0.78 \\
\hline GO:0006839 mitochondrial transport & 73 & 6 & 0.50 \\
\hline $\begin{array}{l}\text { GO:1990542 mitochondrial transmembrane } \\
\text { transport }\end{array}$ & 44 & 3 & 0.41 \\
\hline GO:0008053 mitochondrial fusion & 12 & 3 & 1.52 \\
\hline GO:0000266 mitochondrial fission & 16 & 1 & 0.38 \\
\hline GO:0000422 autophagy of mitochondrion & 27 & 2 & 0.45 \\
\hline $\begin{array}{l}\text { GO:0000002 mitochondrial genome } \\
\text { maintenance }\end{array}$ & 6 & 1 & 1.01 \\
\hline $\begin{array}{l}\text { GO:0009117 nucleotide metabolic process (DNA } \\
\text { replication) }\end{array}$ & 282 & 46 & 0.99 \\
\hline
\end{tabular}

The false discovery rate (FDR) was $>0.0 .5$ for all processes. 


\section{Discussion}

A high mtDNA copy number is critical for normal embryonic development. A key regulator of the mtDNA copy number is TFAM, as TFAM initiates mtDNA replication by its capability to wrap, bend and unwind the mtDNA [8], a function conserved in zebrafish [21]. In order to study the effect of a decreased mtDNA copy number during zebrafish embryonic development, we performed tfam knock-down using a splice-morpholino (MO). We demonstrated that the tfam splice-MO induced skipping of exon 2, leading to a frameshift and a premature stop codon (p.(Cys29Hisfs*36)) (Figure 1A) and to nonsense mediated decay. RNA expression of normal tfam is reduced to $20 \%$ of control values. At 3 and $4 \mathrm{dpf}$, morphant zebrafish have a decreased mtDNA copy number and are less well developed. This correlates with the previous observations that in wild-type zebrafish larvae an increase in mtDNA copy number only becomes apparent between 2 and $3 \mathrm{dpf}$ [10] indicating that, in the absence of mtDNA replication, mtDNA copy number and OXPHOS capacity becomes critically low at 3-4 dpf, as an increase is required for embryonic tissue differentiation and organogenesis. This is a critical phase of development, when there is a high energy demand and a metabolic switch from glycolysis to OXPHOS takes place [22, 23]. This necessity is illustrated by the aberrant eye and muscle development, pericardial oedema and reduced brain size that were observed in morphant zebrafish (Figure 2). The heart and the brain are the first organs to develop [24] and therefore the first to be affected by OXPHOS defects. Moreover, as in a normal heart $>30 \%$ of the cardiac muscle volume is occupied by mitochondria, the deficit of mtDNA (and subsequently mitochondria) also hampers proper alignment of the muscle fibres in the developing heart, creating structural problems as well [25]. In homozygous Tfam KO mice, OXPHOS was abolished due to a severe mtDNA depletion, causing a complete failure of organogenesis and embryonic death [7]. Heterozygous KO mice were viable, but showed an OXPHOS deficiency in the developing heart and a dilated cardiomyopathy and conduction block later in life [7, 26]. The severity of our model seems to fit somewhere in between, as wild-type tfam levels are around $20 \%$ of control level at the day of analysis. Noticeably, MOs establish only a transient knockdown, which is not the case for a homozygous or heterozygous knockout of the gene, prohibiting a proper comparison later in life. 
In order to unravel the biological processes that were altered, we performed global gene expression profiling and subsequent gene ontology (GO) analysis (Table $\mathbf{1}$, Figure 3). Despite the observed reduction in OXPHOS function, the gene ontology term comprising mitochondrial respiratory chain components was not significantly changed, neither were critical mitochondrial processes (Table 2). However, gene expression was decreased of 8 out of the 9 mtDNA protein encoding transcripts, all encoded by the $\mathrm{H}$-strand, whereas the one transcript on the L-strand was unaltered. This most likely this reflects a switch or preferable transcription from the Light-Strand Promoter (LSP) that is induced by low amounts of tfam and which also primes $\mathrm{H}$-strand replication [27], but which in our case fails to increase mtDNA copy number due to the persisting lack of tfam.

The GO term drug catabolism was significantly changed, comprising several downregulated genes linked to mitochondrial function, including embryonic haemoglobin production. As haem is produced by mitochondria [28], this could be explained by the decreased amount of mtDNA and subsequently mitochondria at the onset of definitive haematopoiesis, initiating between 1 to $2 \mathrm{dpf}$ [29-32]. Obviously, deficiencies in haematopoiesis during development might have downstream effects as well. Other mitochondria-related genes of this GO term were decreased, such as oxct1a, oxct $1 b$, members of cyp450 family 2 and gpx $1 a$, which are related to the metabolism of fatty acids and ketone bodies, exogenous drug catabolism and hydrogen peroxide degradation in the mitochondria. The decrease in mtDNA copy number and subsequent mitochondrial dysfunction appears to result in enrichment of GO terms related to rRNA synthesis, ncRNA processing, and ribosomal assembly (Table $\mathbf{1}$ ), and the vast majority of genes in these GO terms was upregulated (Supplementary Table S4). In addition, the GO term tRNA processing was also significantly enriched. In general, expression of genes belonging to these GO terms is increased, including a number of genes that are linked to mitochondrial function, like mto1 (1.64), trmt10c (1.55) and elac2 (1.61) [33-35]. Increased tRNA synthesis was also observed by Torrent et al. upon applying diverse stress conditions to Saccharomyces cerevisiae, demonstrating the regulation of tRNA abundance upon cellular stress [36]. However, future research is needed to identify if the observed activation nucleolar 
processes is caused by mitochondrial stress or is a reaction to compensate for the reduction in mitochondrial transcripts. The resulting disbalance between mitochondrial transcripts and proteins and nuclear encoded proteins that migrate into the mitochondria might be a factors as well. Also, gene expression of 8 out of 9 exosome components, belonging to GO term ribosomal biogenesis, were significantly upregulated in tfam splice-MO zebrafish. Like increased rRNA and tRNA expression, upregulation of the exosome components seems to be a compensatory or stress-induced mechanism, as exosomes contain and shuttle mRNA, and are important in intercellular communication and developmental patterning [37-39]. Future assessment of the zebrafish exosome composition is needed to provide further insight into this novel mechanism by which decreased mtDNA copy number affects exosome signalling and composition.

Since clear morphological abnormalities were apparent in tfam splice-MO zebrafish at $4 \mathrm{dpf}$ (Figure 2), the nucleolar activation upon mitochondrial deficiency was obviously insufficient to compensate. At gene expression level in tfam splice-MO zebrafish at $4 \mathrm{dpf}, \mathrm{GO}$ terms related to vision and sensitivity to light were significantly changed. Retinal development is critical between 32 and 74 hpf and normally largely completed at 4dpf [40]. This window coincides with the decrease in mtDNA copy number in tfam splice-MO zebrafish, explaining the downregulation observed. These extremely down-regulated eye genes are mainly involved in processes and response to light stimulus (Supplementary Table S4), like pde6c (0.05) and gnat2 (0.09). Zebrafish mutants of these genes respectively cause retinal and retinal pigment epithelial disease and cone-rod dystrophy [40, 41]. Additional down-regulated opsins genes related to the photoreceptor function are listed in our zebrafish data (Supplementary Table S4). The lack of significant changes in pathways linked to cardiac, muscle or brain development, suggests that although pathology clearly manifested, we are still observing the processes preceding the pathology and not those resulting from it in the pathway analysis. In addition to defective eye development, a number of temporally expressed developmental genes are downregulated in tfam splice-MO zebrafish at $4 \mathrm{dpf}$, such as chitinase (Chia) 1, 2, 3 and 4. Expression of chia.1, chia.2 and chia.3, which belong to the GO term drug metabolism, normally drastically increases at 4 and $5 \mathrm{dpf}$, while chia.4 is more stably expressed. Chitinases are suggested to play a 
role in early embryo immunity and chitinase inhibition has been shown to cause development disruption in zebrafish, characterized by hampered trunk and tail formation [42].

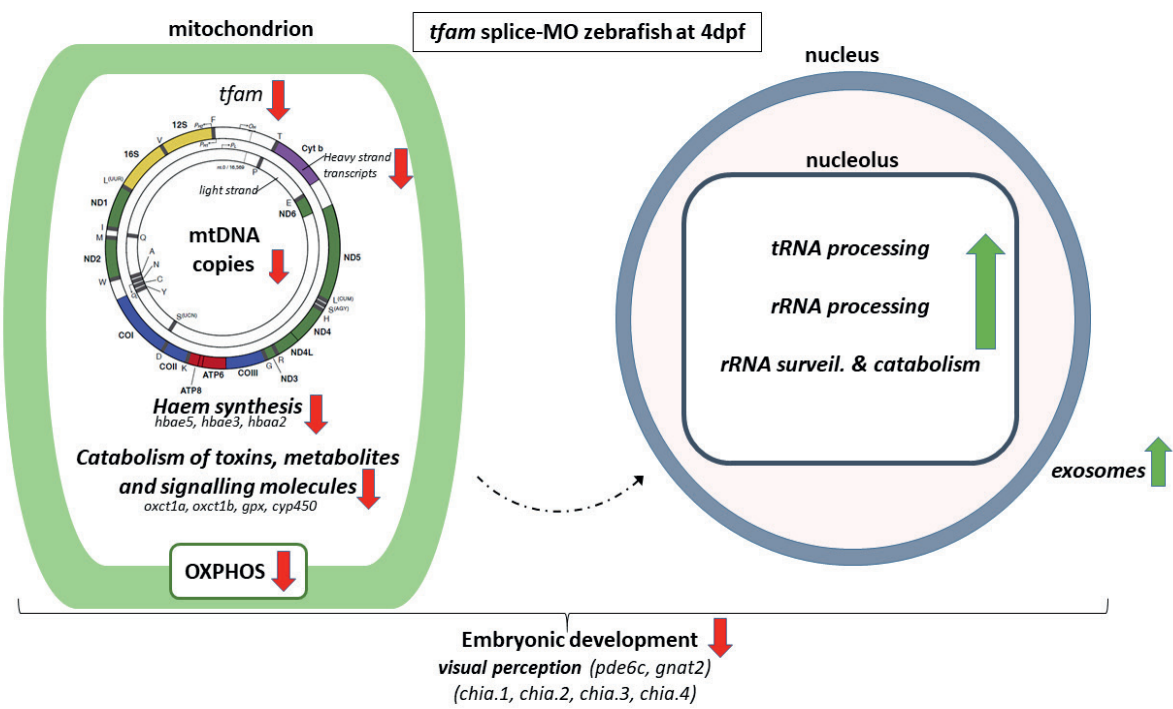

Figure 3. Schematic overview of alterations in tfam splice-MO zebrafish at $4 \mathrm{dpf}$. The arrow indicates if a process is upregulated (green) or down-regulated (red). Altered gene expression processes and genes are shown in italic. The mitochondrial DNA figure is adapted from (Amorim et al. (2019)).

In conclusion, inhibition of tfam expression during early zebrafish development leads to a decrease in mtDNA copy number, OXPHOS capacity, and mtDNA encoded transcripts and haem production, affecting the oxygen supply in the developing embryo. This triggers a compensation mechanism or stress response in the nucleoli at the level of rRNA and tRNA biogenesis and translation initiation, but this compensation mechanism falls short, as pathology emerges (Figure $\mathbf{3}$ ). The results of this study in zebrafish are comparable to other animal models, thereby validating our model, but they also take it a step further due to the clear advantages of the zebrafish model, being a high number of offspring, a rapid development and an optical clarity during development combined with easy genetic interventions. Being able to tune the mtDNA content, we can now 
manipulate the mtDNA bottleneck and study in sufficient numbers the effects on processes, like organogenesis, fertility and mtDNA mutation rate.

\section{Acknowledgements}

The authors would like to acknowledge Jo M. Vanoevelen (MUMC+, Maastricht), Ellen EH Lambrichs (MUMC+, Maastricht), Sabina JV Vanherle (MUMC+, Maastricht), and Levi GK Wackers (Maastricht University, Maastricht) for their technical laboratory skills and practical contribution to this study. M.M. is a "Maître de Recherche", supported by the "Fonds National pour la Recherche Scientifique" (FNRS). This work was further supported by the Interreg IV program of the European Council (the Alma in Silico project to M.M. and H.J.M.S.) and the European Research Area Network for Research Programmes on Rare Diseases 2 project GENOMIT (grant R 50.02.12F to M.M.). Part of this work has been made possible with the support of the Dutch Province of Limburg (M.G., and H.S.) and the Research School GROW (A.O., P.L., H.S.). This manuscript has been released as a Pre-Print at BioRxiv 843318v1 [43].

\section{Funding}

M.M. is a "Maître de Recherche", supported by the "Fonds National pour la Recherche Scientifique" (FNRS). This work was further supported by the Interreg IV program of the European Council (the Alma in Silico project to M.M. and H.J.M.S.) and the European Research Area Network for Research Programmes on Rare Diseases 2 project GENOMIT (grant R 50.02.12F to M.M.). Part of this work has been made possible with the support of the Dutch Province of Limburg (M.G., and H.S.) and the Research School GROW (A.O., P.L., H.S.). 


\section{Authors Contribution Statement}

F.T. and H.S. designed and supervised the project and shared joint last authorship in the manuscript. A.O. and R.K. designed, performed most experiments, coordinated collaborations with other authors (P.L., M.G., H.P., M.M.), and A.O. and R.K. wrote as shared first joint authorship in the manuscript. P.L. designed, assigned, and supplied transcriptomic computational analysis. R.K., F.T. and H.S. determined PANTHER pathway gene-expression analysis on transcriptomic data. Further, Tfam knockdown experiments as quantitative and qualitative analysis, imaging embryos, oxygen consumption rate measurements were performed by A.O., R.K. in the manuscript. M.G. assisted on the design and scientific input on the manuscript. H.P., and M.M. assisted on the design and scientific input in the manuscript and were coordinating the housing and injections of the zebrafish embryos. All authors contributed ideas to the project.

\section{Conflict of Interest Statement}

The authors declare that the research was conducted in the absence of any commercial or financial relationships that could be construed as a potential conflict of interest.

\section{Contribution to the Field Statement}

The first tuneable zebrafish model used to characterize the effect of a reduced mtDNA copy number and resulting OXPHOS deficiency on zebrafish embryonic development.

\section{Data Availability Statement}

All microarray data is deposited in the GEO database, accession GSE146696. 


\section{References}

1. Otten, A.B. and H.J. Smeets, Evolutionary defined role of the mitochondrial DNA in fertility, disease and ageing. Hum Reprod Update, 2015.

2. Santos, T.A., S. El Shourbagy, and J.C. St John, Mitochondrial content reflects oocyte variability and fertilization outcome. Fertil Steril, 2006. 85(3): p. 584-91.

3. Ebert, K.M., H. Liem, and N.B. Hecht, Mitochondrial DNA in the mouse preimplantation embryo. J Reprod Fertil, 1988. 82(1): p. 145-9.

4. Wai, T., et al., The role of mitochondrial DNA copy number in mammalian fertility. Biol Reprod, 2010. 83(1): p. 52-62.

5. Yamamoto, T., et al., Effect of maternal age on the developmental competence and progression of nuclear maturation in bovine oocytes. Mol Reprod Dev, 2010. 77(7): p. 595-604.

6. Parisi, M.A., B. Xu, and D.A. Clayton, A human mitochondrial transcriptional activator can functionally replace a yeast mitochondrial HMG-box protein both in vivo and in vitro. Mol Cell Biol, 1993. 13(3): p. 1951-61.

7. Larsson, N.G., et al., Mitochondrial transcription factor $A$ is necessary for mtDNA maintenance and embryogenesis in mice. Nat Genet, 1998. 18(3): p. 231-6.

8. Ekstrand, M.I., et al., Mitochondrial transcription factor $A$ regulates mtDNA copy number in mammals. Hum Mol Genet, 2004. 13(9): p. 93544.

9. Pauli, A., et al., Antisense Oligonucleotide-Mediated Transcript Knockdown in Zebrafish. PLoS One, 2015. 10(10): p. e0139504.

10. Otten, A.B., et al., Differences in Strength and Timing of the mtDNA Bottleneck between Zebrafish Germline and Non-germline Cells. Cell Rep, 2016. 16(3): p. 622-30.

11. Artuso, L., et al., Mitochondrial DNA metabolism in early development of zebrafish (Danio rerio). Biochim Biophys Acta, 2012. 1817(7): p. 100211.

12. Larbuisson, A., et al., Fgf receptors Fgfr1a and Fgfr2 control the function of pharyngeal endoderm in late cranial cartilage development. Differentiation, 2013. 86(4-5): p. 192-206.

13. Kimmel, C.B., et al., Stages of embryonic development of the zebrafish. Dev Dyn, 1995. 203(3): p. 253-310.

14. Bill, B.R., et al., A primer for morpholino use in zebrafish. Zebrafish, 2009. 6(1): p. 69-77.

15. Atlas, Z. Zebrafish Atlas Slides. 2019, 8 August; Available from: http://bio-atlas.psu.edu/zf/progress.php.

16. Akaike, H. Information theory and an extension of the maximum likelihood principle. in Second International Symposium on Inference Theory. 1973. Budapest: Akadémiai Kiadó. 
17. Ihaka R, G., R "R: a language for data analysis and graphics". Journal of Computational Graphics and Statistics, 1996. 5(3): p. 299-314.

18. Lindsey, J., Models for repeated measurements, 2nd edition. 1999, Oxford: Oxford University Press. 536.

19. Thomas, P.D., et al., PANTHER: a library of protein families and subfamilies indexed by function. Genome Res, 2003. 13(9): p. 2129-41.

20. Mi, H., et al., PANTHER version 11: expanded annotation data from Gene Ontology and Reactome pathways, and data analysis tool enhancements. Nucleic Acids Res, 2017. 45(D1): p. D183-D189.

21. Howe, K., et al., The zebrafish reference genome sequence and its relationship to the human genome. Nature, 2013. 496(7446): p. 498503.

22. Hance, N., M.I. Ekstrand, and A. Trifunovic, Mitochondrial DNA polymerase gamma is essential for mammalian embryogenesis. Hum Mol Genet, 2005. 14(13): p. 1775-83.

23. Stackley, K.D., et al., Bioenergetic profiling of zebrafish embryonic development. PLoS One, 2011. 6(9): p. e25652.

24. Glickman, N.S. and D. Yelon, Cardiac development in zebrafish: coordination of form and function. Semin Cell Dev Biol, 2002. 13(6): p. 507-13.

25. Ventura-Clapier, R., et al., Bioenergetics of the failing heart. Biochim Biophys Acta, 2011. 1813(7): p. 1360-72.

26. Powell, C.A., T.J. Nicholls, and M. Minczuk, Nuclear-encoded factors involved in post-transcriptional processing and modification of mitochondrial tRNAs in human disease. Front Genet, 2015. 6: p. 79.

27. Shutt, T.E., M. Bestwick, and G.S. Shadel, The core human mitochondrial transcription initiation complex: It only takes two to tango. Transcription, 2011. 2(2): p. 55-59.

28. Wagener, F.A., et al., Different faces of the heme-heme oxygenase system in inflammation. Pharmacol Rev, 2003. 55(3): p. 551-71.

29. Murayama, E., et al., Tracing hematopoietic precursor migration to successive hematopoietic organs during zebrafish development. Immunity, 2006. 25(6): p. 963-75.

30. Bertrand, J.Y., et al., Definitive hematopoiesis initiates through a committed erythromyeloid progenitor in the zebrafish embryo. Development, 2007. 134(23): p. 4147-56.

31. Zhang, X.Y. and A.R. Rodaway, SCL-GFP transgenic zebrafish: in vivo imaging of blood and endothelial development and identification of the initial site of definitive hematopoiesis. Dev Biol, 2007. 307(2): p. 179-94.

32. Zhang, J. and I. Hamza, Zebrafish as a model system to delineate the role of heme and iron metabolism during erythropoiesis. Mol Genet Metab, 2018.

33. Fakruddin, M., et al., Defective Mitochondrial tRNA Taurine Modification Activates Global Proteostress and Leads to Mitochondrial Disease. Cell Rep, 2018. 22(2): p. 482-496. 
34. Haack, T.B., et al., ELAC2 mutations cause a mitochondrial RNA processing defect associated with hypertrophic cardiomyopathy. Am J Hum Genet, 2013. 93(2): p. 211-23.

35. Metodiev, M.D., et al., Recessive Mutations in TRMT10C Cause Defects in Mitochondrial RNA Processing and Multiple Respiratory Chain Deficiencies. Am J Hum Genet, 2016. 99(1): p. 246.

36. Torrent, M., et al., Cells alter their tRNA abundance to selectively regulate protein synthesis during stress conditions. Sci Signal, 2018. 11(546).

37. Wan, J., et al., Mutations in the RNA exosome component gene EXOSC3 cause pontocerebellar hypoplasia and spinal motor neuron degeneration. Nat Genet, 2012. 44(6): p. 704-8.

38. Boczonadi, V., et al., EXOSC8 mutations alter mRNA metabolism and cause hypomyelination with spinal muscular atrophy and cerebellar hypoplasia. Nat Commun, 2014. 5: p. 4287.

39. McGough, I.J. and J.P. Vincent, Exosomes in developmental signalling. Development, 2016. 143(14): p. 2482-93.

40. Richardson, R., et al., The zebrafish eye-a paradigm for investigating human ocular genetics. Eye (Lond), 2017. 31(1): p. 68-86.

41. Brockerhoff, S.E., et al., Light stimulates a transducin-independent increase of cytoplasmic Ca2+ and suppression of current in cones from the zebrafish mutant nof. J Neurosci, 2003. 23(2): p. 470-80.

42. Semino, C.E. and M.L. Allende, Chitin oligosaccharides as candidate patterning agents in zebrafish embryogenesis. Int J Dev Biol, 2000. 44(2): p. 183-93.

43. Auke BC Otten, R.K., Patrick Lindsey, Mike Gerards, Hélène PendevilleSamain, Marc Muller, Florence HJ van Tienen, Hubert JM Smeets, Tfam Knockdown Results in Reduction of mtDNA Copy Number, OXPHOS Deficiency and Abnormalities in Zebrafish Embryos. 2019. 


\section{Tables and Figures}

Supplementary data due to many presented columns in Excel (Table S2, S4, and S5) processed in download link:

https://www.frontiersin.org/articles/10.3389/fcell.2020.00381/full\#supplementa ry-material

Supplementary Table S1. Overview of primers and antibodies used in this study

\begin{tabular}{|c|c|c|c|}
\hline Primers & Forward Primer $\left(5^{\prime}-3^{\prime}\right)$ & Reverse Primer (5'-3') & \\
\hline 18S RNA & $\begin{array}{l}\text { AGCGTGCGGGAAACCA- } \\
\text { CGAG }\end{array}$ & AAGCCGCAGGCTCCACTCCT & \\
\hline Tfam Exon 2 & $\begin{array}{l}\text { TGTGTAGCTCCAGCGA- } \\
\text { TAAAGA }\end{array}$ & $\begin{array}{l}\text { ATGTATGCTGTCAGGG- } \\
\text { GTCTCT }\end{array}$ & \\
\hline Tfam Exon 5 & $\begin{array}{l}\text { CCCAAGGAGCACCTT- } \\
\text { CAACA }\end{array}$ & TTGCCTGCGTAGTAGTTCCC & \\
\hline Tfam Exon 6-7 & $\begin{array}{l}\text { CGCTTGAGCGACACA- } \\
\text { CAAAA } \\
\end{array}$ & GTGCTCCTCCCACGATTTGA & \\
\hline Tfam Exon 1 & $\begin{array}{l}\text { CTTTTGTTCGGTGGT- } \\
\text { CAACA }\end{array}$ & & \\
\hline Tfam Exon 3 & & CTITTGTTCGGTGGTCAACA & \\
\hline Tfam Exon 4 & & TCCGAATGGCTITTCTITG & \\
\hline Tfam Exon 5 & & CGGACTTTATCGTCCTCTGC & \\
\hline ND1 & $\begin{array}{l}\text { CCACTTAATTAACCCCC- } \\
\text { TAGCC }\end{array}$ & ATGTITGTGGGGGTAGACCA & \\
\hline B2M & $\begin{array}{l}\text { CGCCTGAAAACTACGTTC- } \\
\text { TACAC }\end{array}$ & ACTTTCGGAGTGGCTGAAAA & \\
\hline Antibody & Company & Dilution & Incubation \\
\hline $\begin{array}{l}\text { Primary-anti- } \\
\text { MF20-mouse }\end{array}$ & $\begin{array}{l}\text { DSHB, Myosin Heavy } \\
\text { Chain Pro }\end{array}$ & $1: 20$ dilution & $\begin{array}{l}4^{\circ} \mathrm{C} \text { incubation } \\
\text { overnight }\end{array}$ \\
\hline $\begin{array}{l}\text { Primary- } \\
\text { anti-Zebrin } \\
\text { I-mouse }\end{array}$ & $\begin{array}{l}\text { DSHB, Aldolase C like } \\
\text { Protein }\end{array}$ & $1: 100$ dilution & $\begin{array}{l}4^{\circ} \mathrm{C} \text { incubation } \\
\text { overnight }\end{array}$ \\
\hline $\begin{array}{l}\text { Secondary- } \\
\text { anti-mouse/ } \\
\text { rabbit }\end{array}$ & $\begin{array}{l}\text { Dako REAL EnVision De- } \\
\text { tection System Peroxida- } \\
\text { se/DAB }\end{array}$ & Ready to use & $\begin{array}{l}\text { room-tempera- } \\
\text { ture incubation } \\
\text { for } 30 \text { minutes }\end{array}$ \\
\hline
\end{tabular}

Table S2. Analysed transcripts analysed in microarray analysis (use download link) 
Table S3. Gene expression of mtDNA protein encoding genes

Supplementary Table S3. 13

mtDNA protein encoding genes

\begin{tabular}{|c|c|c|c|}
\hline Mapped Ids & $\begin{array}{c}\text { Gene Name/ } \\
\text { Symbol }\end{array}$ & Fold Change & STRAND \\
\hline ENSDARG00000063895.3 & mt-nd1 & 0,75 & H-Strand \\
\hline ENSDARG00000063899.3 & mt-nd2 & 0,69 & H-Strand \\
\hline ENSDARG00000063914.3 & mt-nd3 & 0,72 & H-Strand \\
\hline ENSDARG00000063916.3 & mt-nd4l & 0,67 & H-Strand \\
\hline ENSDARG00000063917.3 & mt-nd4 & 0,60 & H-Strand \\
\hline ENSDARG00000063921.3 & mt-nd5 & 0,58 & H-Strand \\
\hline ENSDARG00000063922.3 & mt-nd6 & $\mathbf{1 , 0 0}$ & L-Strand \\
\hline ENSDARG00000063905.3 & mt-cox1 & 0,62 & H-Strand \\
\hline ENSDARG00000063908.3 & mt-cox2 & 0,71 & H-Strand \\
\hline ENSDARG00000063912.3 & mt-cox3 & not on array & H-Strand \\
\hline ENSDARG00000063910.3 & mt-atp8 & not on array & H-Strand \\
\hline ENSDARG00000063911.3 & mt-atp6 & not on array & H-Strand \\
\hline ENSDARG00000063924.3 & mt-cytb & not on array & H-Strand \\
\hline
\end{tabular}

Table S4. Significantly altered PANTHER GO biological processes including genes (Fold Changes) per group (use download link)

Table S5. Mitochondria-related PANTHER GO biological processes including genes (Fold Changes) per group (use download link) 


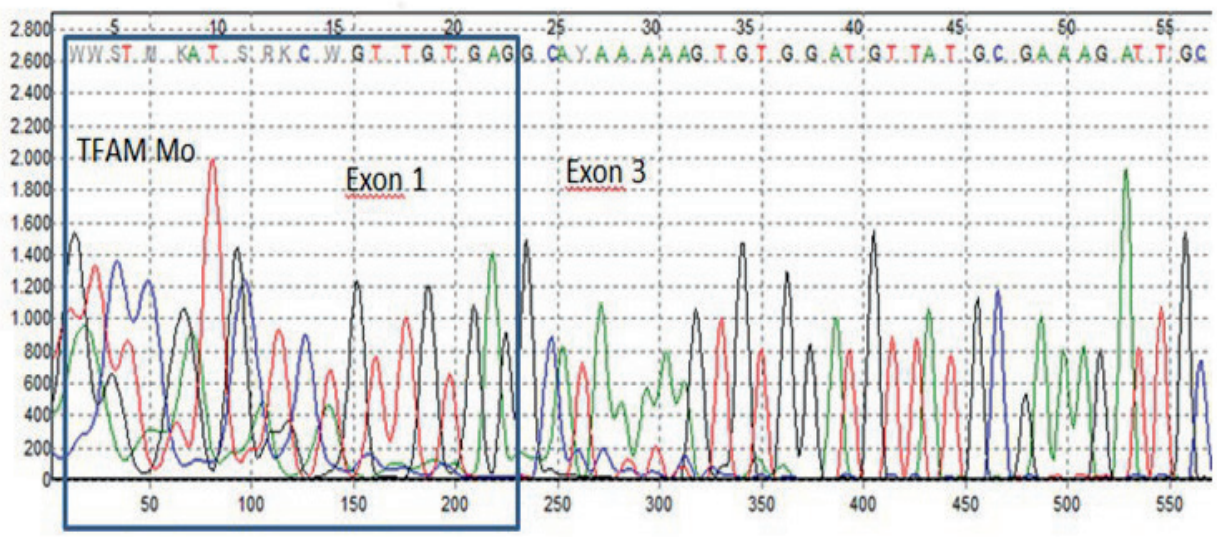

Fig. S1 Sanger sequencing of tfam. Sanger sequencing of tfam after PCR amplification of DNA isolated from tfam splice-MO-treated zebrafish $(n=6$ per injected condition from gene expression analysis samples) showed deletion of exon 2 c.84_211del, which predicts a frameshift and premature stopcodon on protein level (p.(Cys29Hisfs*36)).

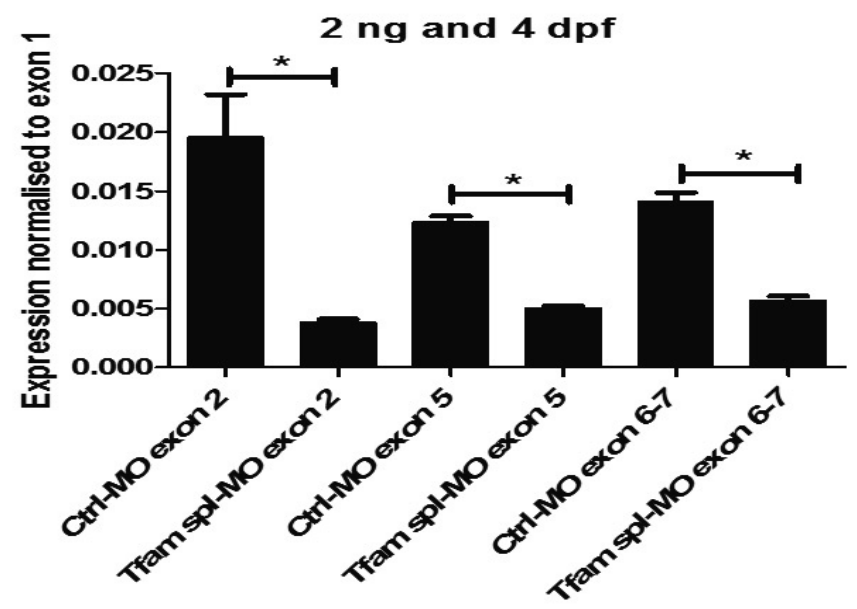

Fig. S2 Q-PCR analysis of total tfam expression. qPCR analysis of total tfam expression showed a 5.3 fold decrease in exon 2 expression (range $1.5-19.7$ ) at day 4 in zebrafish injected with $2 \mathrm{ng}$ tfam splice-MO compared to the Ctrl-MO ( $\mathrm{n}=6$ per injected condition from gene expression analysis samples). Analysis of tfam exon 5 and exon 6-7 showed respectively $41 \%$ and $40 \%$ expression in tfam splice-MO injected embryos at day 4 . This indicates that $\sim 60 \%$ of tfam RNA is subjected to nonsense-mediated decay and that only half of the residual $40 \%$ tfam RNA is exon 2 containing wild-type RNA. Bars indicate mean values with SEM. $P$-values are calculated using Bonferroni's Multiple Comparison Test. $* P$-value $<0.05$. 

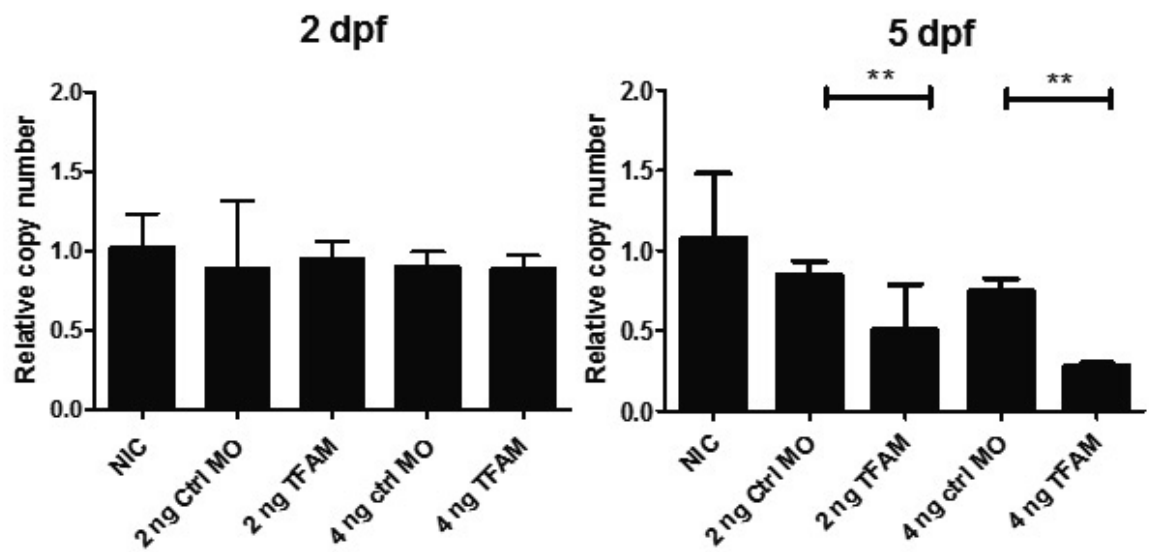

Fig. S3 mtDNA effect of tfam knockdown on the mitochondrial DNA copy number. The relative mitochondrial DNA copy number has been assessed by mitochondrial ND1 / nuclear B2M ratio at $2 \mathrm{dpf}$ and $5 \mathrm{dpf}$ in non-injected control (NIC, $\mathrm{n}=8$ per time point) embryos, 2 or $4 \mathrm{ng}$ control-morpholino-injected (ctrl-MO) embryos and 2 or $4 \mathrm{ng}$ Tfam splice-MO injected embryos ( $n=20$ per injected condition). Data are normalized to the NIC embryos. Bars indicate mean values with $\mathrm{SD}$. $P$-values are calculated using Bonferroni's Multiple Comparison Test. $* P$-value $<0.05, * * P$-value $<0.01$.

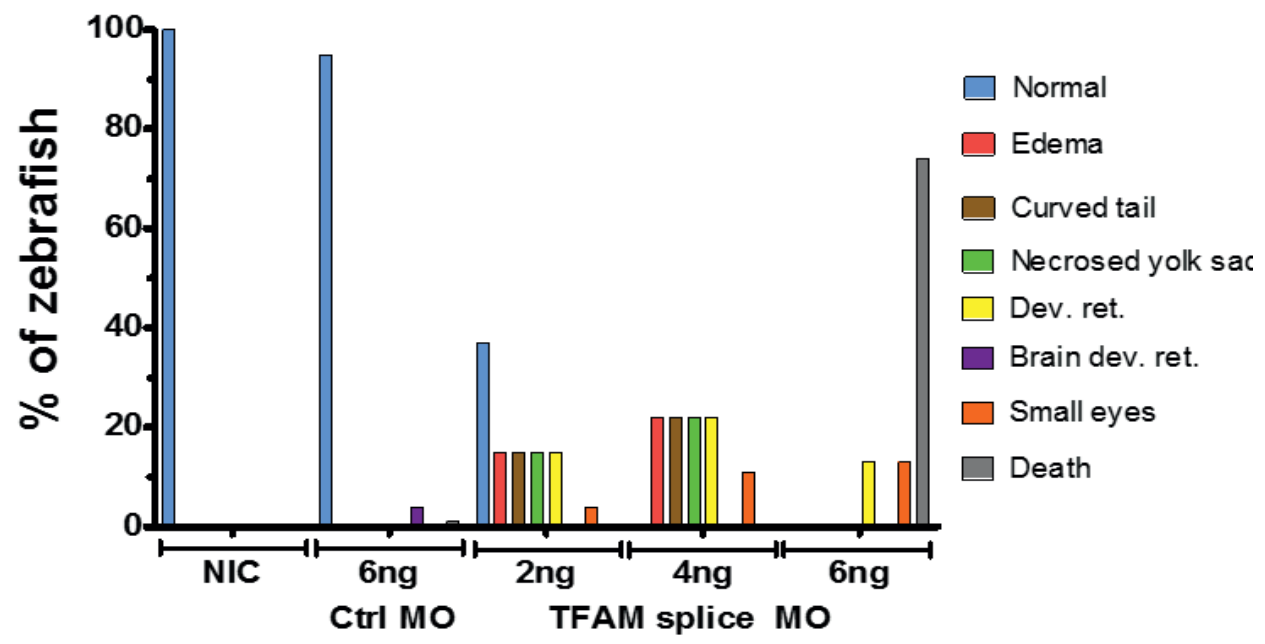

Fig. S4 The macroscopic phenotype scoring. The macroscopic phenotype ( $n=100$ per condition) included overall oedema, curved tails, necrotized yolk sacs and small eyes. Fish injected with $4 \mathrm{ng}$ tfam splice-MO were more severely affected, as they had a higher count for oedema, curved tails, necrosed yolk sac, small eyes, and developmental delay. The percentage of dead embryos was $<1 \%$ for both concentrations of tfam MO-injections at $4 \mathrm{dpf}$. 

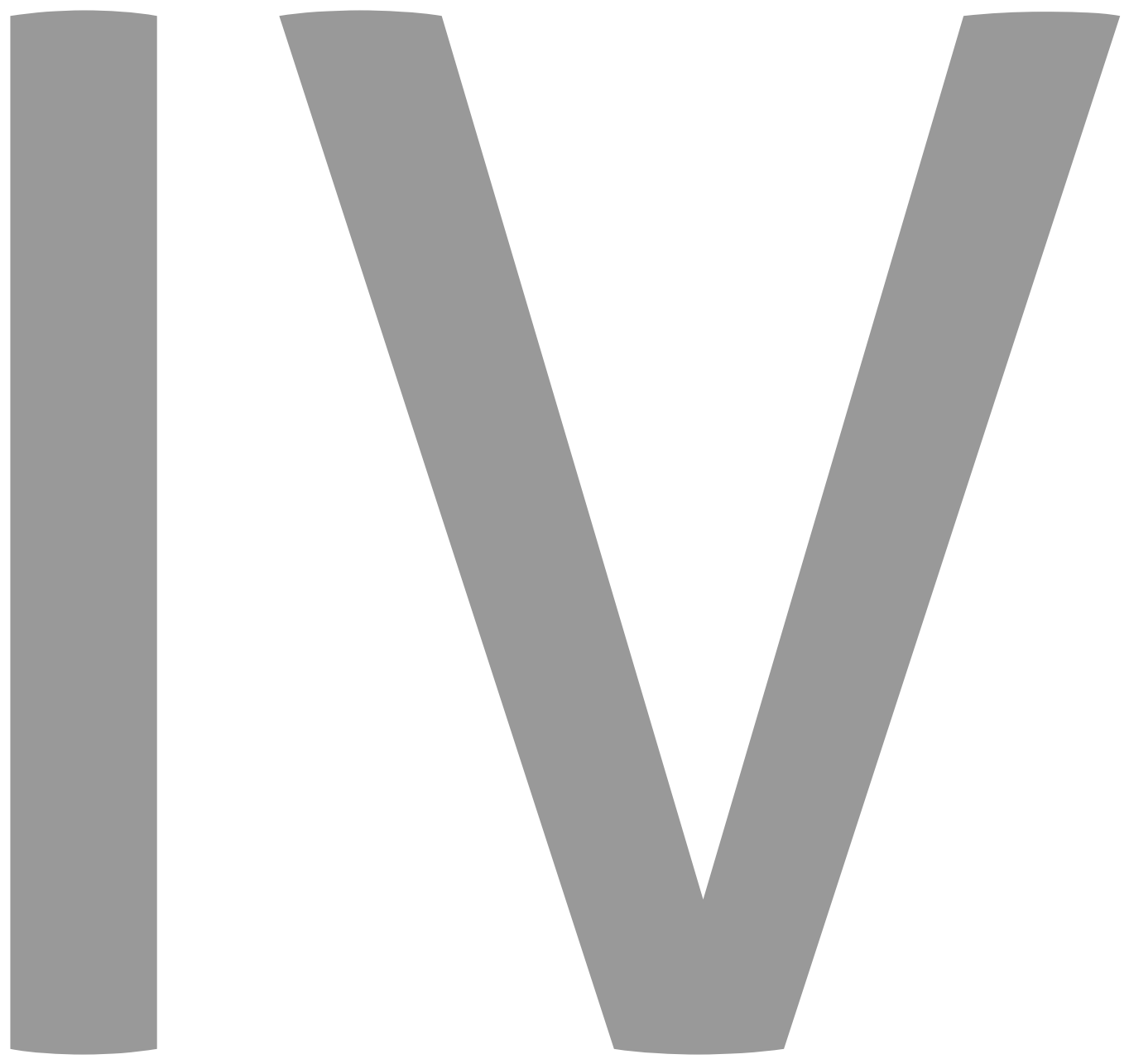
General Discussion and Summary 
CHAPTER 7
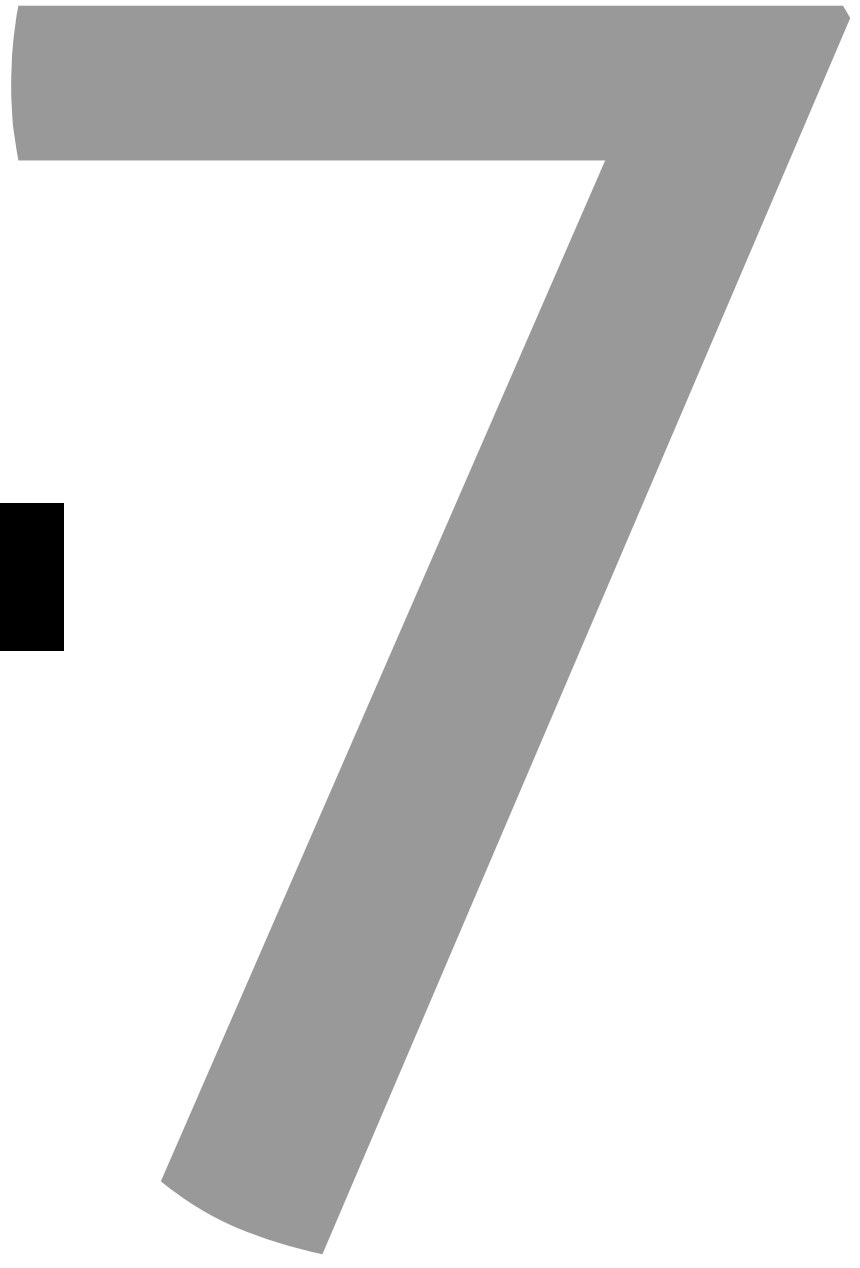
General Discussion 


\section{The Difficulty in Establishing a Genetic Diagnosis in Mitochondrial Disease}

Difficulties in establishing a genetic diagnosis are due to the clinical and genetic heterogeneity of mitochondrial diseases. Extensive evaluation of clinical symptoms, biochemistry, immunohistochemistry, and quality or quantity of the mitochondria are usually not sufficient to pinpoint a specific genetic cause. Since two genomes (mtDNA and nDNA) are involved with possibly more than 1,500 genes, it is evident that finding the genetic cause can be a real odyssey for clinicians and patients $[1,2]$.

\section{Identifying genetic variants in mitochondrial disease}

The work presented in this thesis demonstrates the impact next-generation sequencing (NGS) and more specific whole-exome sequencing (WES) has on the genetic diagnostics of mitochondrial disease [3]. A diagnostic rate of $25 \%$ was common in pre-NGS days at the start of the work, presented in this thesis, resulting from an extensive, costly, and time-consuming usually Sanger-based sequencing process of sequentially selected individual candidate genes $[4,5]$. Family studies and linkage analysis were fundamental to characterize unknown genes in combination with functional assays. Application of NGS resulted in a diagnostic yield of $69 \%$ in our cohort of patients with mitochondrial and neuromuscular disease (Chapter 2), consisting of $20 \%$ pathogenic mtDNA variants and $49 \%$ pathogenic nuclear gene variants [3]. No laboratory assays were required to select candidate genes, only assays were needed for validation of unclassified variants in known genes and pathogenic variants in novel genes. In these cases, functional, complementation assays were required to prove pathogenicity and link the genetic variant to the phenotype (Chapter 4-5). In two previous studies using targeted nuclear mitochondrial gene panels the diagnostic yield was $22 \%$ [6] and $24 \%$ [7], which is less than in our study, screening all nuclear genes (49\%). This can be explained by incompleteness of the mitochondrial panels, as up to $30 \%$ of the disease-causing genes identified in our patient cohort were not present in the MitoCarta database at the time of discovery. A previous NGS-based study using WES in 109 paediatric and young adult patients with clinical characterized mitochondrial disease showed a diagnostic yield of $39 \%$, closer to our $49 \%$ [8]. Another mitochondrial disease 
study resulted in a diagnostic yield of 59\% after screening both mtDNA and WES, compared to our $69 \%$ [9]. A possible explanation is that we included only wellcharacterized patients who were likely to have an autosomal recessive inherited gene defect (consanguineous parents or multiple affected siblings with healthy non-consanguineous parents). As consanguineous parents have a higher risk of having children that suffer from multiple genetic diseases, we emphasize that WES data should be screened completely in those cases and investigations should not be stopped by finding a single genetic defect to which the entire clinical manifestation is attributed. As this has been done in the past, one should carefully evaluate genotype-phenotype correlations for over-interpretation of a single gene defect. The same is true for patients from non-consanguineous parents when a combination of genetic defects is more likely than a single gene defect. Then the whole exome should be analysed. We demonstrated for a few patients that they can carry more than one genetic defect and that for prognosis and prevention of transmission it is crucial to identify all possible causes [10].

Nowadays, genetic defects causing mitochondrial diseases can be found in any of the processes, involved in mitochondrial structure, stability and function. In Chapter 5, we described a number of novel defects in genes (QRSL1, MTO1, and $A A R S 2)$ in WES data, involved in the mitochondrial translation process, in which the brain (Leigh-like syndrome) and the heart (HCM) were prominently affected [11]. During our studies, two sibling with HCM were reported having a diseasecausing genetic defect in the QRSL1 gene. A second unrelated non-HCM patient harboured compound-heterozygous mutations in the QRSL1 gene causing interstitial pneumonia and mitochondrial respiration deficiencies, showing the complexity and variability of disease progression between patients in time with the same gene affected [12]. Furthermore, we reported two novel variants in the MTO1 gene in a paediatric patient with early-onset HCM, causing a diffuse cerebral atrophy with an intraventricular septum, and multiple OXPHOS deficiencies, which was lethal at the age of 19 months. An unrelated patient with a homozygous MTO1 mutation showed HCM without lethality, and normal brain development. Finally, we identified two variants in the AARS2 gene, manifesting with paediatric cardiomyopathy, Leigh-like syndrome, and multiple OXPHOS deficiencies in the patient. Although, multiple pathogenic AARS2 variants have been reported in 
literature, resulting in hypertrophic cardiomyopathy and lethality after 3 months of birth [13, 14], other AARS2 patients presented with leukodystrophy, and ovarian failure. In general, mitochondrial translational defects lead to a broad variety of clinical manifestations, of which the underlying tissue-specific mechanisms can not be fully explained yet [15] (Figure 1).

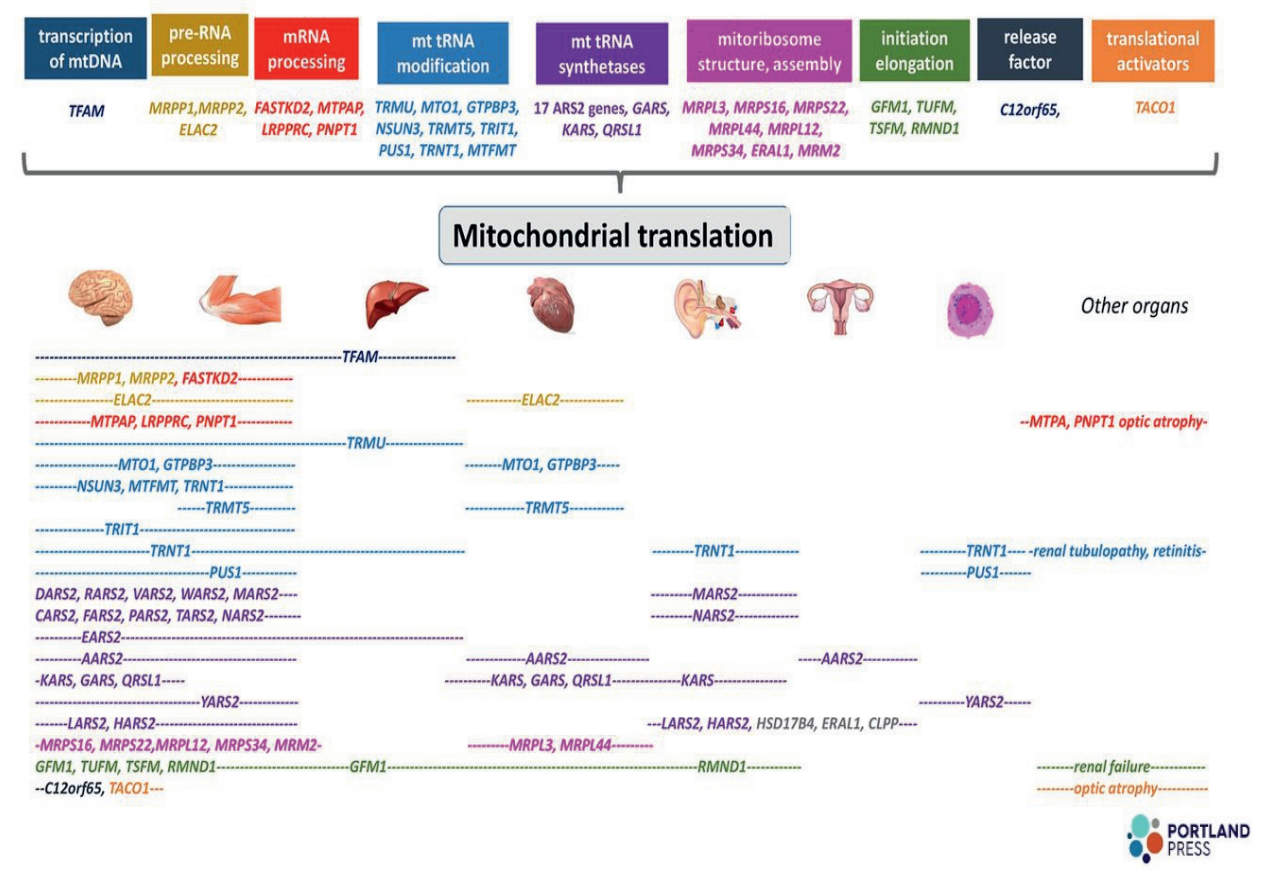

Figure 1. Summary of the genes and disease mechanisms implicated in mitochondrial translation deficiencies with associated clinical phenotypes, Picture adapted from: Veronika Boczonadi et al. Essays Biochem. 2018;62:321-340.

Part of the variability of clinical manifestations can be attributed to the involvement of multiple genes. Especially, heterogeneous clinical phenotypes may not always be caused by a monogenic defect, especially in consanguineous families, and it is important to follow-up all pathogenic variants that can be related to the clinical symptoms. Cases with multiple underlying genetic causes have been reported with overlapping (blended) or separate (composite) clinical symptoms [10]. These patients suffer from multiple genetic diseases. Alternatively, the variation could be caused by so-called genetic risk factors that modify the clinical manifestation of the primary gene defect. A clear example for this, is the 
m.3243A>G mutation, which can lead to Mitochondrial Encephalopathy, Lactic Acidosis, and Stroke-like (MELAS) syndrome, but also can manifest with diabetes, epilepsy, cardiac arrhythmia, hearing loss, kidney problems, and even acute collapse, and death [15] separately or in combination. This can not be explained by differences in mutation load only and additional genetic or environmental factors, like physical exercise, seem to be involved [15]. Leber's Hereditary Optic Neuropathy (LHON) is an example of reduced penetrance, as it is caused by, in general, homoplasmic mutations in the mtDNA, but only $50 \%$ of the males and $10 \%$ of the females suffer from visual loss. Again, genetic and environmental factors, like smoking, seem to be involved. A WES study revealed an interaction between the mtDNA mutation (m.11778G $>A$ ) and a homozygous mutation (c.572G > T, p.191Gly > Val) in the YARS2 as a nuclear-modifier gene of the severity of vision loss [16]. Furthermore, LHON have been associated with other neurological symptoms and multiple sclerosis, but a for the latter it may also be a coincidental observation [17]. Genome-wide association studies (GWAS) have been performed in common diseases to identify genetic risk factors, however, large sample size and replication cohorts are required, which are not available for mitochondrial diseases as such [18]. Still, the identification of genetic risk factors and the current strategy to convert them cumulatively in polygenic risk scores (PRS) could explain a considerable part of the missing variability in common disease, which could be expanded to mitochondrial disease [19] as example, a PRS study in Parkinson Disease (PD) revealed that cumulatively, small effect variants in primary and secondary mitochondrial genes are significantly associated with increased PD risk. It would be worthwhile to check if these variants could also be involved in the clinical variability observed in primary mitochondrial disease. It would also be worthwhile to study if part of the remaining cases with mitochondrial disease, currently without genetic diagnosis, could be explained by a high PRS, based on multiple small effect variants.

As said before, the high diagnostic yield (69\%) in our cohort (Chapter 2) might result from a selection for patients with a highly likely genetic cause and the ability to perform functional tests for validation. The substantial part of novel disease genes still identified and the overlapping clinical phenotypes, makes panel-based or targeted NGS cumbersome and less effective for mitochondrial diseases. An 
unbiased approach is highly preferable for these genetically and clinically heterogeneous disorders. Still, a genetic diagnosis is lacking in $31 \%$ of the cases. These patients might have mutations, missed by the recessive disease model used, and could carry for example de novo mutations, which have been checked only for the clinical exome. Including WES analysis of the parents and perform a trio analysis, could easily solve this issue [20]. However, de novo mutations will not explain the entire missing group. As WES analysis is limited to the proteincoding region (exons) of the genome and to a few intronic nucleotides, which are the most conserved splice sites adjacent to the exon, WES does not cover the remaining of the introns, promoter regions and non-coding RNAs. In addition, computer-based tools and pipeline analyses are less accurate in predicting the effect of DNA-variants on gene-expression or RNA splicing. Therefore, mutations with a potential pathogenic effect on RNA expression or processing are either not included in the regions of interest in WES sequencing or are missed by bioinformatics analysis [21-24]. Sequencing the transcriptome by RNA-Seq is therefore a valuable addition to detect variants affecting RNA levels and composition. RNA-Seq allows analysis of the transcriptome at an unprecedented depth [25-28]. Recently, a publication reported the identification of causative, non-exonic regulatory variants in an additional $10 \%$ of mitochondrial patients using RNA-Seq [29]. In another study of rare muscular disorders this was 35\%, which clearly shows the added value of this approach. However, one should realize that by RNA-Seq only the genes are interrogated, which are expressed in the tissue of investigation, which might limit the success rate for diseases, in which the affected tissue cannot be easily collected.

Whole Genome Sequencing (WGS) has the potential to solve issues of missing variants and it will be a matter of time, before WGS will be the standard in diagnostics. Apart from analysing the entire genome, the major technical advantage of WGS is that the library preparation does not require any enrichment or amplification and the specificity is theoretically $100 \%$ (achieving around $95 \%-$ $98 \%$ in practice) with a uniform coverage throughout the input material. Therefore, the possibility to miss a disease-causing variant (or any other information) for technical reasons such as inefficient probe targeted enrichment, inefficient amplification of a specific ROI or PCR amplification artefacts do not 
apply to WGS [30-32]. WGS, using long-read sequencing, is the best approach for detecting all variants, as short-read sequencing still misses repeats and structural genomic variants. However, long-read is more expensive and more error prone than short-read sequencing and also the throughput is considerably less, making a combination of long-read and short-read sequencing currently the preferred strategy for comprehensive genome sequencing.

Although WGS data are still limited compared to WES, a number of successes have been reported. In a proband with possible GSD-1a, WGS long-read sequencing identified a causal structural variant, being a $7.1 \mathrm{~kb}$ deletion (covering two exons on the first allele) in the G6PC gene in a WES negative case. An additional missense variant; c.326G>A; p.(C109Y) was reported on the second allele [33]. A second study used paired-end short-read and direct long-read WGS to investigate an unresolved autosomal dominant dementia family linked to 7q36. A chromosomal inversion of ca. $4 \mathrm{Mb}$ was identified and validated by long-read sequencing, which disrupted the coding sequence of the DPP6 gene, being a novel gene causing dementia [34]. Genetic diseases are the leading cause of infant mortality in Western countries, particular in infants admitted to paediatric and cardiovascular intensive care units (ICUs). Rapid WGS (rWGS) showed a diagnostic yield of 42 to $57 \%$ in serious ill infants in ICUs, and changes in medical management in 30 to $72 \%$, and finally altered outcomes in 24 to $34 \%$ of the cases [35]. This retrospective study reported a successful diagnosis in three out of seven seriously ill ICU infants [35]. rWGS successes in these cases obviously accelerate the implementation of WGS in clinical diagnostics and care. In 2016 a Dutch Consortium (RADICON-NL), consisting of 8 Academic Genetic Centres, 2 nonAcademic Neonatal Intensive Care Units (NICU's) and several patient organizations, also started with WGS for this patient group [36].

Worldwide many large-scale WGS projects have been started. WGS has been shown to be instrument for cancer care and therapy. The International Cancer Genome Consortium (ICGC) and The Cancer Genome Atlas (TCGA) report on the presence of somatic mutations in different solid tumours to define an optimal, personalized treatment strategy $[37,38]$. The 100,000 Genomes Project is a UK Government project that is sequencing whole genomes from National Health Service patients. The project is focusing on rare diseases, some common types of 
cancer, and infectious diseases. The project was initiated in 2012 to develop an infrastructure for massive routine use of WGS. The medical and genomic data is shared with researchers, to improve knowledge of the causes, treatment and care of diseases [39]. Other countries that have initiated national genome projects are Japan (Initiative on Rare and Undiagnosed Diseases), China (100,000 Genomes project), Australia (Australian Genomics Health Futures Mission), Saudi Arabia (Saudi Human Genome Program), United Arab Emirates (Dubai Genomics), Turkey (Turkish Genome Project; 100,000 Genomes) and Estonia (Personalized Medicine Program; 100,000 Genomes). The French Plan for Genomic Medicine 2025 started in 2016. By 2020, 235000 genomes will be sequenced each year in France, primarily for cancer and rare diseases. The largest WGS project has been initiated in the United States (All of Us Research Program; 1,000,000 Genomes) [40]. This does not focus on rare diseases or cancer, it is a precision medicine initiative aimed to enrol a diverse group of one million people. Participants submit their medical records to the National Institutes of Health, fill out online surveys about their lifestyles, furnish blood and urine samples, and have their genomes sequenced. The Genome of the Netherlands (GoNL) project was launched in 2012, in which they sequenced whole genomes of 250 Dutch parent-offspring families and constructed a haplotype map of 20.4 million single-nucleotide variants and 1.2 million insertions and deletions. The intermediate coverage $(\sim 13 x)$ and trio design enabled extensive characterization of structural variation, including midsize events (30-500bp) previously poorly catalogued and de novo mutations [41].

It is beyond any doubt, that WGS will bring the paradigm shift started by WES to the next level: for a genetic diagnosis, geneticists and expert clinicians will no longer need to narrow down the differential clinical diagnosis to request sequencing of a specific gene or sets of genes, but all genetic information will be available at once from start. The results of this genetic analysis will directly guide the diagnostic patient evaluation, moving towards personalized patient care and treatment. Still, a drawback of whole genome sequencing as addressed in the next paragraph is the large amounts of variants detected, for which bioinformatics or functional approaches to characterize their significance are not fully developed yet [42]. 


\section{Interpreting genetic variants in mitochondrial disease}

To identify the genetic cause of a disease in an affected patient, it is essential to assign clinical significance to the variants identified. First, one can check if the variant has been reported before and how the variant had been classified. Several databases (e.g. OMIM [43], LOVD [44], ClinVar [45]) are available and list variants and their reported pathogenicity. Alternatively, one can predict the pathogenicity using a variety of bioinformatics tools, which can predict the effect of a variant on the protein level, the protein activity, RNA splicing, and ultimately help to assess the presence of protein damaging alterations. Alamut [46], Polyphen [47], Sift [48] are all suitable variant prediction tools. This analytical process is either performed manually for each variant or can be automated. International guidelines are available to assist such classification [49]. The scoring system for mtDNA mutations in protein-encoding genes is comparable to these accepted criteria of pathogenicity [50], in which reported mtDNA variants with a score of 6 or less points were considered neutral polymorphisms, between 7 and 10 points possibly pathogenic, between 11 and 13 points probably pathogenic, and 14 or more as definitely pathogenic, with a maximum of 20 . The criteria included; more than one independent report, evolutional conservation of the base or base-pair, variant heteroplasmy level, segregation of the mutation with disease, histochemical evidence of mitochondrial disease, biochemical defects in complexes (I, III, or IV), evidence of mutation segregation with biochemical defect from single-fiber studies, or evidence of pathogenicity in trans-mitochondrial cybrid studies (see below). The pathogenicity scoring system was similar for mitochondrial tRNA mutations, including specific characteristics of the tRNA and excluding criteria only relevant for proteins [51]. This system was refined in later publications $[52,53]$. Co-segregation of the variant with the disease in the family further supports a possible role, but the ultimate proof of the pathogenicity of a variant would be a second unrelated, phenotypically similar patient or functional confirmation (see below). An updated list of mtDNA point mutations and their classification can be found in MitoMAP [54] (http://www.mitomap.org/MITOMAP), the Mamit-tRNA database [55], (http://mamit-trna.u-strasbg.fr/), in HGVS (www.hgvs.org/mutnomen/), and in mtDB (http://www.mtdb.igp.uu.se/). The 
reference mtDNA sequence is the revised Cambridge Reference Sequence (rCRS), GenBank number NC_012920.1.

The systematic screening of mtDNA and nDNA using in silico prediction tools and pathogenicity scores revealed a substantial number of novel variants of unknown significance (VUS). About $6.5 \%$ of the mitochondrial patients carries a VUS, creating uncertainty and anxiety [56]. Trans-mitochondrial cybrid studies can be used to functionally characterize mtDNA mutations that cause a measurable OXPHOS defect in fibroblasts and to determine the threshold of expression [57]. Cybrids are cytoplasmic hybrid cell lines. Cells devoid of mtDNA (rho zero cells) are fused with patient-derived mitochondria from fibroblasts with an OXPHOS deficiency. OXPHOS measurements in the cybrids will reveal whether the transfer of mitochondria from the patient induced the same OXPHOS deficiency or not. In that case an mtDNA defect is likely the cause. In this way, the pathogenicity of many mtDNA mutations was functionally established [57].

For classification of potential novel disease-causing genes, functional assays are essential. For mitochondrial disease, (electron) microscopy (EM), biochemical (ATP, lactate), and/or enzyme measurements (e.g. respiratory chain complex deficiency) can show abnormalities, which could either be linked to the genetic defect or used for complementation assays (Chapter 4-5), which is particularly easy in case the enzyme deficiency is also present in patient-derived cells. Currently, also 600 metabolites can be measured quantitative in metabolomics studies or even more than $\sim 5,400$ proteins in proteomics [58] or the complete mitochondrial proteome analysis [59]. A successful integration of metabolomics showed to be useful for variant classification of exome data in the spermine synthase gene (SMS) that causes Snyder-Robinson syndrome (SRS). Untargeted plasma metabolome analysis revealed significantly elevated levels of $\mathrm{N}^{8}$ acetylspermidine, a precursor derivative of spermine biosynthesis, as a potential novel plasma biomarker for SRS [60]. This study illustrates the potential of metabolomics as a translational technique to support exome data on a functional and clinical level in case of clinical exome sequencing identified a novel mutation or gene. Finally, transgenic animal models (mice, zebrafish) can be constructed to relate the genetic defect to clinical or morphological manifestations. 


\section{Treatment and Prevention of Mitochondrial Disease}

Despite considerable efforts in developing therapies, still no cure exists for mitochondrial diseases in general. Several strategies have been investigated, ranging from physical exercise and diets, to small molecules and gene therapy $[61,62]$. No broadly accepted, validated biomarkers exist to identify mitochondrial pathology and monitor the efficacy of the interventions. FGF-21 seems promising as it can be elevated in both mtDNA and nDNA encoded mitochondrial disease $[63,64]$. As a limitation, FGF-21 also seems be elevated in other conditions, including obesity and fastening [65]. Furthermore, the identification of the gene defect in the patient has not led to a major breakthrough in novel treatments. Only in few cases, a gene specific-treatment is possible and our studies and others show that riboflavin can be beneficial for patients with ACAD9 gene defects [6668], high-fat diet for those with TMEM126B gene defects [69] and thiamine for those with SLC19A3 gene defects as reported in Chapter 4, [70-72]. Early start with these treatments prevents symptoms from occurring and can be lifesaving, for example, by preventing fatal Leigh Syndrome in patients with SLC19A3 mutations.

If not treatable, the aim is to prevent further transmission of mitochondrial diseases. As mitochondrial diseases have a dual genetic origin, this has implications for the transmission and recurrence risks. Nuclear gene defects may inherit in autosomal recessive or X-linked fashion or in autosomal dominant modus, but also de novo dominant variants are quite frequent (16\%) and should be considered. Generally available options, like prenatal diagnosis (PND) or preimplantation genetic diagnosis (PGD), currently referred to as Prenatal Genetic Testing (PGT) apply, although these can not prevent de novo disease. For mtDNA mutations the situation is different. The mtDNA is transmitted by a mother to her children and during oogenesis a drastic decrease in mtDNA copy occurs, which is followed by an amplification of this subset in maturing oocytes, the so-called mitochondrial genetic bottleneck (Figure 3). In carriers of a heteroplasmic mtDNA mutation, due to this bottleneck, the mtDNA mutation load can vary considerably among the oocytes of such a carrier and in the resulting offspring [73]. Currently, if a patient carries an mtDNA mutation as the cause of disease, three situations can apply with respect to recurrence risk. If the mutation is de novo in the patient, 
which is the case for about $25 \%$ of patients with an mtDNA disease, the recurrence is low, as the mother does not carry the mutation, and prenatal diagnostics (PND) is offered for reassurance [74]. If the mother is carrier of a heteroplasmic mutation, preimplantation genetic diagnostics (PGD) can be offered and unaffected embryos with a mutation load below the threshold of expression can be transferred [75]. In case of a homoplasmic mutation or a very high mutation load in the mother, it is impossible or unlikely to select healthy embryos, mitochondrial replacement therapy (MRT) is the method of choice. Currently MRT is offered in the UK for severe mtDNA disease only, in which PGD is unlikely to be successful [76], https://www.umdf.org/mitochondrial-replacement-therapy/.

Despite the high impact the bottleneck has on the transmission of mtDNA disease, the underlying mechanism is still poorly understood. The size of the bottleneck remains an issue of debate and if positive or negative selection occur on mutations in addition to random genetic drift and at which level is largely unknown. Recently, in a human study available oocytes, zygotes, and blastomeres of five carriers of the $M T T L 1 ; \mathrm{m} .3243 A>\mathrm{G}$, one carrier of the ATP6;m.8993T>G, and one carrier of the ND6;m.14487T>C mutation were analysed for the mtDNA mutation load. Genetic drift with a variable bottleneck could explain the distribution, but it was far more likely that selection mechanisms could play a role as well [77]. The absence of mutation loads above $80-90 \%$ for $M T T L 1 ; \mathrm{m} .3243 \mathrm{~A}>\mathrm{G}$, which could not be explained by random segregation, indicated that at these levels OXPHOS function was insufficient for oogenesis, which heavily relies on OXPHOS. It has been reported that above $90 \%$ OXPHOS assembly is lost (Complex I) and $>80 \%$ ATP production almost drops to zero. The ATP6;m.8993T>G shows in general either very high or very low levels of the mutation load. This binomial distribution was long enigmatic, but might be explained by a combination of genetic drift and positive selection on the mutation. At high mutation loads (up to $100 \%$ ) ATP production drops to $20-30 \%$, which seems sufficient for proper oogenesis. As this can not explain why high mutation loads would be preferred, we proposed, based on data [77], that the mutation leads to a high membrane potential, which due to the defect in complex $V$ is not consumed for ATP production. As a high membrane potential is usually a hallmark of healthy mitochondria, this could reflect a selection mechanism based on the membrane potential. The ND6;m.14487T>C 
showed a random distribution with no apparent selection, which is consistent with the mutation being milder than the m.3243A>G. Based on this data, the mitochondrial bottleneck and genetic drift can be complemented with mutationspecific negative or positive selection mechanism. Probably, these mutationspecific events should be evaluated for each mtDNA mutation as the potential of preferential segregation of mutated mtDNA can have consequences for the threshold of transfer in case of PGD and the risk that mtDNA carry over can lead to clinical consequences $[78,79]$.

A number of limitations prevents an accurate study of transmission of mtDNA disease in humans. The main issue is the availability of oocytes and embryos to study the transmission of mtDNA [80]. Due to these limitations, multiple family studies have been performed in order to get indirect information on the segregation of mtDNA. These studies have revealed random segregation and selection alike [81-83]. It is evident that further insight in these mechanisms might have implications for the reproductive choices and the chances of having unaffected offspring. If selective mechanisms can be identified, these might also reveal possible therapeutic interventions.

\section{The Use of Animal Models to Study the mtDNA Bottleneck}

Animal models have provided insight in the occurrence of mtDNA variants and the mechanism by which they are either filtered out or spread to high levels in the entire body, the germ cells or in a specific organ upon segregation. The mechanism of the bottleneck during development seems to be well-conserved although the amounts of mtDNA in oocytes differ largely among animal models [73]. In any case, the mature oocyte is the cell with the highest number of mtDNA copies and mitochondria in each species [73]. The mtDNA copy numbers is tightly regulated during oogenesis and development, in line with the metabolic demands of the cell. The mtDNA is also packed together with its proteins into nucleoids. Each nucleoid contains one or two mtDNA molecules [84]. Based on studies in animals a number of different mechanisms were postulated for the mtDNA bottleneck to explain the changes in heteroplasmy levels observed $[85,86]$. In Holstein cows it was reported that de novo occurring, transmitted heteroplasmic variants could become fixed as homoplasmic variants within three generations 
[87]. This was explained by the first hypothesis for the mitochondrial bottleneck, which postulated that only a small proportion of the total mtDNA passed on from mother to offspring, assuming genetic drift as the driving force.

Rodents have higher numbers of offspring, making them more suitable for studying the bottleneck, but the number of strains carrying an mtDNA mutation is still small [88]. A commonly used model to study the transmission of mtDNA variants is the 'mutator mouse', a model with many mtDNA mutations due to a proofreading-deficient mitochondrial DNA polymerase (Polga). In the offspring, the mtDNA mutations are randomly distributed and can accumulate clonally in somatic cells, causing a mosaic of respiratory chain deficiencies in tissues such as heart, skeletal muscle and brain. Moreover, analysis of these tissues has shown that mtDNA mutator mice contain an increased load ( 3-5 times) of the somatic point mutations in the mtDNA and an increased amount of eliminated mtDNA. Eventually, the increase in somatic mtDNA mutations is associated with a reduction in the life-span and early onset of aging-related phenotypes [86]. As the number of mutations is too high to study the mitochondrial bottleneck, a mice model was derived from the mutator mouse, carrying only a single heteroplasmic mutation (Figure 2). A strong reduction of the amount of mtDNA was reported in 2 independent studies in primordial germ cells $[89,90]$, however, the results were interpreted quite differently. In the first paper [87] a mathematical stimulation was used to predict the mtDNA bottleneck, based on random genetic drift and a single mitochondrion/mtDNA molecule as segregating unit. A minimal amount of approximately $\sim 200$ mtDNA copies could explain the variation in heteroplasmy seen in the offspring of heteroplasmic mice [89]. About $70 \%$ of the heteroplasmy variance was credited to the physical restriction of mtDNA levels in early PGCs and the remaining $30 \%$ was due to a rapid proliferation in PGCs during maturation. The conclusion was that the mtDNA bottleneck consisted of unequal segregation of a drastically reduced mitochondrial genome population in early post implantation development (Figure 2a). So, the unequal partitioning (segregation) of mutated (red) and wild-type (blue) genotypes and resulting shifts in heteroplasmy in oocytes is explained by the strong reduction in mtDNA copy number immediately prior to expansion of the primordial germ cell (PGC) population [87]. The second paper also reported a reduction of mtDNA copy 
number in PGCs at 8.5 days in mice, but a limited number of heteroplasmy measurements did not appear to show an increase in heteroplasmy variance in the early PGCs. Therefore, the authors concluded that the variation in heteroplasmy does not develop until maturation in the primary follicles. They proposed that mtDNA molecules were packaged into homoplasmic nucleoids, or simply kept separate within discrete homoplasmic mitochondria, thereby reducing the number of segregating units and accelerating the rate of drift (Figure $\mathbf{2 b}$ ) [90]. Alternatively, as a third explanation, the replication of a subpopulation of mitochondrial genome would also lead to a bottleneck effect (Figure 2c). There is some evidence that this might occur during postnatal oocyte maturation [90, 91]. Further experiments are needed to show, which of the predicted models can explain the data best.
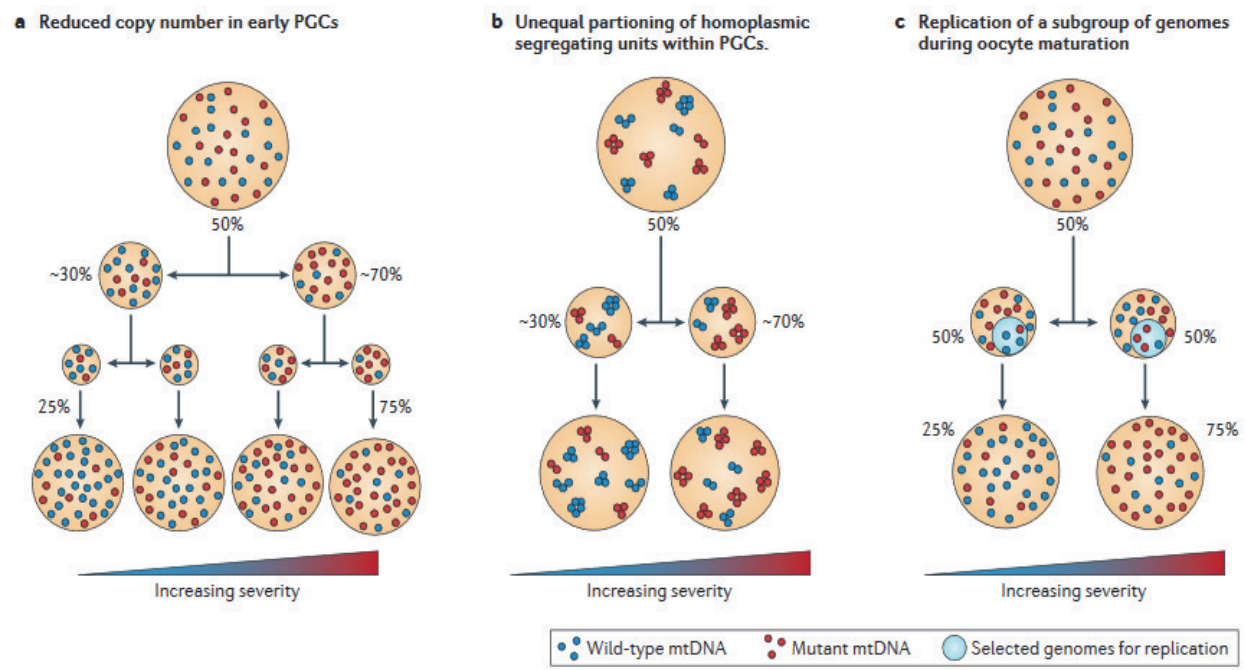

Figure 2. The dynamics of mitochondrial DNA heteroplasmy: implications for human health and disease. Picture adapted from Stewart JB, Chinnery PF, Nat Rev Genet. 2015 Sep;16(9):530-42.

All 3 mechanisms assumed that segregation of mtDNA mutations would only depend on random genetic drift. This can also explain the segregation in families by postulating a variable sized, mutation-dependent bottleneck [81-83]. However, as mentioned above, evidence was reported for selection processes during oogenesis and embryogenesis in humans and mice models. A mice model containing two mutations (ND6;m.13885insC and COI;m.6589T>C) that affect 
oxidative phosphorylation showed different segregation, which could be explained by selection occurring [92]. The severe ND6 mutation was clearly selected against and was eliminated within four generations. The milder COI mutation was still present throughout multiple generations, showing genetic drift and no apparent selection on the mutation despite symptoms of myopathy and cardiomyopathy later in life.

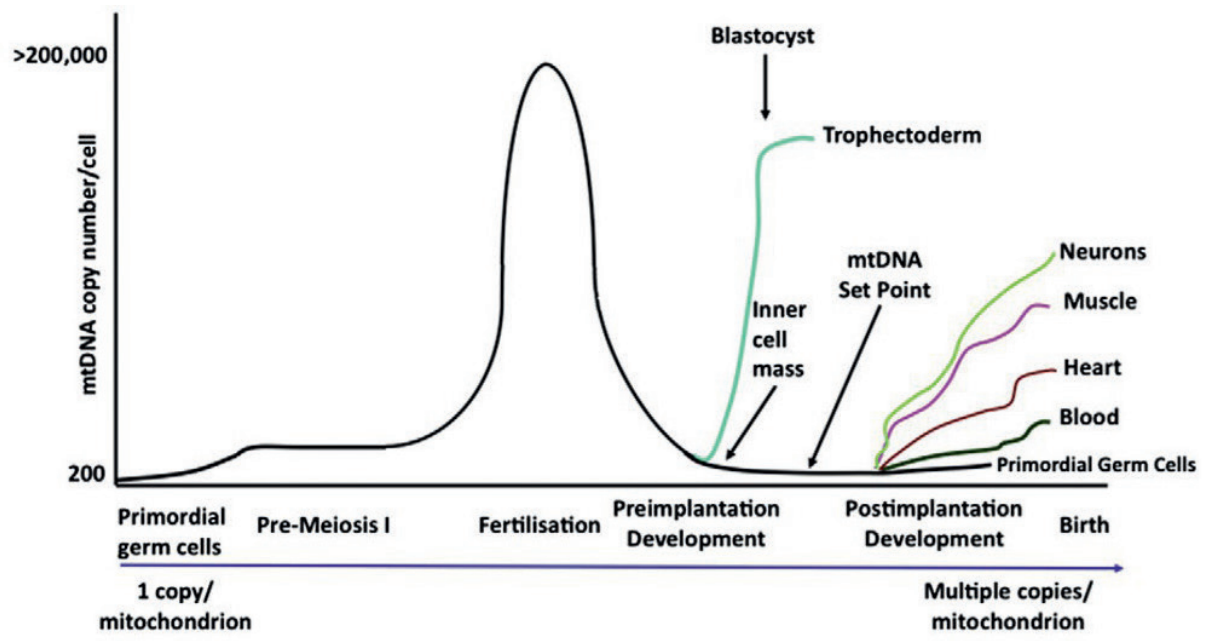

Figure 3. The mtDNA copy number per cell during embryonic and foetal development in mammals. During oogenesis, mtDNA copy number increases up to the metaphase II stage. In larger animals, it then undergoes significant reduction during preimplantation development. At the blastocyst stage, replication is initiated, but this is confined to the trophectoderm. mtDNA copy number undergoes a continual reduction in the inner cell mass cells to establish this mtDNA set point which guarantees that specialised cell types once they have differentiated, acquire the appropriate mtDNA copy number to meet their needs for ATP production in a cell specific manner (adapted from: St John et al in 2014. The control of mtDNA replication during differentiation and development. Biochim Biophys Acta, 2014. 1840(4): p. 1345-54.

Studies on the mitochondrial bottleneck are still hampered by the lack of data and of appropriate animal models with heteroplasmic mtDNA mutations in which the bottleneck can be manipulated. Zebrafish are potentially an attractive model, as it is possible to isolate at low costs large numbers of oocytes, primordial-germcells, and somatic embryonic cells to characterize the mitochondrial bottleneck during early embryonic development. Potential factors underlying this bottleneck can be easily manipulated, as shown for TFAM in Figure 4 [93]. Different levels of TFAM can lead to differences in mtDNA replication and transcription of the light 
and heavy strand. Based on mtDNA and transcript levels of mtDNA encoded transcripts, the zebrafish tfam knockdown as described in Chapter 6, most likely reflects a switch or preferable transcription from the Light-Strand Promoter (LSP), which is induced by low amounts of tfam and which also primes $\mathrm{H}$-strand replication[93]. It can be expected that overexpressing of Tfam will lead to an increased mtDNA copy number [94], which will allow us to manipulate mtDNA copy number in both directions. A drawback of the zebrafish model is the current lack of fish with heteroplasmic mtDNA mutations, which would allow us to perform similar studies as has been done for a mouse model with a heteroplasmic mtDNA mutation, in which Tfam was either overexpressed or knocked-out [87]. Heteroplasmic mutations do exist in the mtDNA in oocytes of zebrafish although generally at low level [85]. Our next aim is to develop heteroplasmic zebrafish models to study the bottleneck, the role of the specific mutations and possible selection mechanisms in further detail.
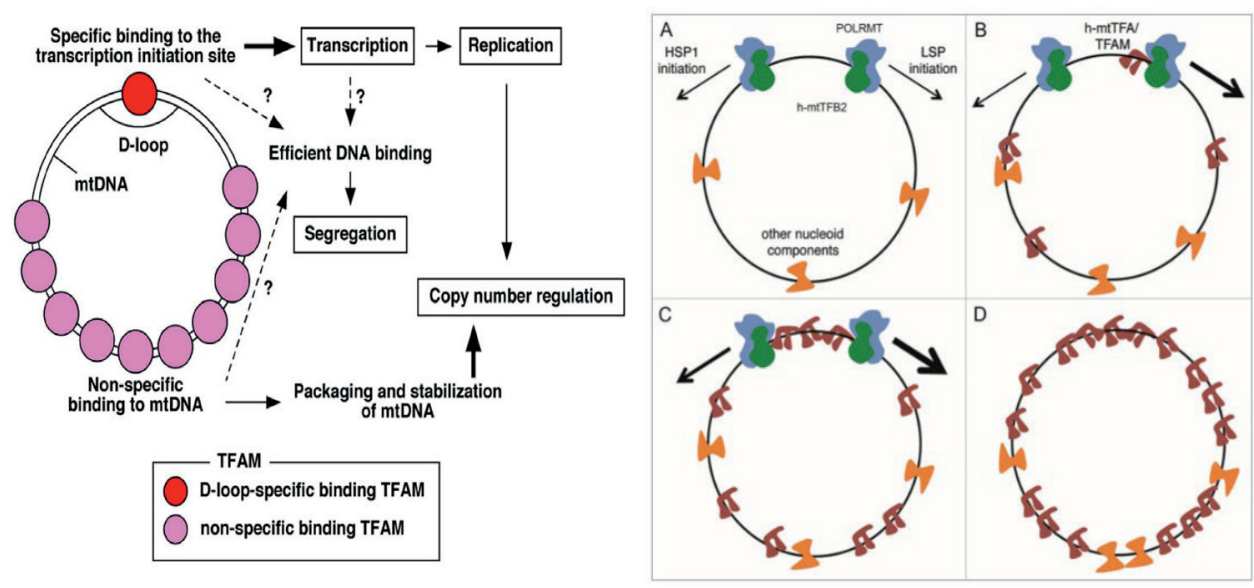

Figure 4. The core human mitochondrial transcription machinery. The hypothetical model of transcription initiation determined by the amount of mammalian mtTFA associated with the number of mtDNA molecules per nucleoids. In a schematic representation of an mtDNA molecule (Circle in black) including the two basal components of the transcription process (in blue POLRMT and in green human-mtTFB2) bound at the HSP1 and LSP promoter sites. The arrow thickness represent the amount of transcription initiation activity from both strands. The human mtTFA in red and figure shape is representing other nucleoid components as the ones in orange bound to the mtDNA. This red and orange situation symbolizing different nucleoids containing several mtDNA molecules (AD states). (A) An mtDNA state with very little or no h-mtTFA bound is shown to visualize that basal levels of transcription from both promoters could be present in this situation. (B) An mtDNA state with relatively low amounts of $h$-mtTFA bound, but importantly bound at the high-affinity LSP site where it initially activates transcription of this promoter preferentially. Although, transcription from the LSP also primes $\mathrm{H}$-strand mtDNA 
replication, a switch occurs from a state akin to state $A$ towards a state $B$, which could be a switch to activate mtDNA replication. (C) An mtDNA state with significantly higher amounts of h-mtTFA bound that leads to a significant activation of both promoters. In this various states in this range of $h$-mtTFA saturation could result in dynamic regulation of the relative amounts of HSP1 and LSP transcription. (D) A quiescent mtDNA state represent extreme high level or even saturation levels of h-mtTFA amounts, which would predict in silencing of transcription and replication. So in mammals, cells could have similar states in vivo, with heterogeneity potentially occurring at the level of cell or either tissue type, or individual organelles or maybe even at level of each nucleoid as adapted from Shutt et al in 2011. The core human mitochondrial transcription initiation complex - It only takes two to tango. Transcription 2:2, 55-59; March/April 2011.

\section{Role of the mtDNA Bottleneck beyond mtDNA Disease Transmission}

As the mitochondria content per cell declines during blastomere formation, the glucose transporter expression increases and glucose uptake accelerates to increase glycolysis activity in the blastocyst. Although, the transition in mitochondria and energy metabolism is well known during early embryogenesis, less information is reported on the metabolic maturation of specific organs or the role for mitochondria in fertility and successful embryonic development (Figure 3). Organ specific development can be modelled in vitro by using stem cell differentiation, which is corroborated by a metabolic transition from predominantly glycolysis to mitochondrial dependent oxidative phosphorylation (OXPHOS) [95] as shown in Figure 5. Embryonic stem cells (ES) derived from the inner mass of the blastocyst, are excellent models to study either the differentiation process into multiple tissue-specific lineages or self-renewal by the possibility to self-replicate while in the undifferentiated state. The shape of the mitochondria in ES is similar as in the fertilized oocyte, spherical and poor cristae development, but in contrast, only contains a low mtDNA copy number. Unbalanced mitochondrial homeostasis in ES results in loss of pluripotency and lower efficiency in differentiation capacity and ES maintenance. The bioenergetics requirements of pluripotency can also be studied in induced pluripotent stem cells (iPS) cell lines, derived from somatic cells. During reprogramming a massive mitochondrial restructuring is shown with a shape transition from mature cristae rich mitochondria (somatic phenotype) towards immature poor shaped mitochondria with reduced mtDNA copy number in iPS cells. This mitochondrial reprogramming results in a decrease of cellular respiration in iPS cells providing a switch from somatic OXPHOS metabolism into glycolysis. These observations suggest that glycolytic activity of pluripotent stem 
cells is a critical factor to maintain this pluripotent state, but that metabolic energy capacity and mitochondrial activity are essential for the differentiation process. Establishing a comprehensive roadmap of all cellular bioenergetics during developmental stages would offer a fundamental metabolic guide in the energetic requirements of somatic and germ-line cells, which is important for reproduction and healthy development [100-102], but also for tissue regeneration [95].

As shown in Figure 3 during early embryogenesis the mtDNA copy number undergoes significant reduction, affecting both germ line and somatic cells and tissues [96]. In line with what has been described above, differentiation and organogenesis lead to mtDNA replication and a switch to OXPHOS, at first observed in the trophectoderm. A somatic bottleneck also occur often in cancer and tumorigenesis, where a mtDNA copy number set-point has to be crossed for further tumor progression [97]. The critical role for a somatic bottleneck in increasing the effect of subclinical mtDNA mutations is demonstrated in HIV patients treated with anti-retroviral drugs (NRTIs). This treatment leads to a reversible reduction in the amount of mtDNA (mtDNA depletion) and a progressive accumulation of the load of somatic mtDNA mutations, which cause age-related mitochondrial disease in these patients at an earlier age [98].

Especially in IVF the role of mitochondria and mitochondrial copy number has been studied to select embryos for successful implantation with somehow controversial observations [99]. A sufficiently high mtDNA copy number appears to be important for successful oogenesis and development [100]. As a consequence oocytes without sufficient wild-type mtDNA would not have the capacity to generate enough ATP to mature, which prohibits healthy embryonic development [97]. Therefore, the direct relationship between mtDNA copy number in the oocyte and its viability was proposed [99]. A possible relation between mtDNA content of human blastocysts, female age, embryonic or chromosomic status, and implantation studied in 379 IVF embryos, of which NGS data from preimplantation genetic screening (PGS) revealed the opposite [100]. Blastocysts that successfully implanted tended to contain lower mtDNA quantities than those failing to implant independent from age. Also, aneuploid blastocysts contained significantly higher amounts of mtDNA than euploid samples. This observation was confirmed in a second study by another group [106], analysing 290 euploid 
embryos of a group of 270 patients undergoing preimplantation genetic screening (PGS) before single embryo transfer. Implantation and pregnancy rates were calculated. This study showed that an increased amount of mtDNA in euploid embryos is indeed related to poor implantation potential. This data seems contradictory to the observation that sufficient mtDNA is required for oogenesis and embryogenesis, but it could be possible that a too low mtDNA copy number in oocytes triggers mtDNA replication earlier than normal during embryogenesis, leading to an increased mtDNA copy number in the blastocyst with advancing age [101]. However, in 2017, this data was challenged when a third study showed that human blastocysts contained uniform levels irrespective of ploidy, or age, or implantation potential [102]. In addition, this study raised concerns on the methodology used in the previous reports [100,103]. Especially, their critique on technical protocols used to determine mtDNA copy number from NGS data of preimplantation genetic testing was heavily debated and did not lead to consensus among all groups involved. Also, the the way the nuclear genome should be taken into account as the embryo gender and ploidy can undermine the calculated mtDNA scores, was a topic of fierce discussion. Another interfering factor is that the collection of viability data on embryos is performed in a reference laboratory, but the data is collected from multiple centers with subsequently different protocols (e.g. culture media, method of collecting biopsies, equipment, or personnel). So, the issue of mtDNA copy number as a marker for successful implantation is still not resolved and awaits further confirmation. 


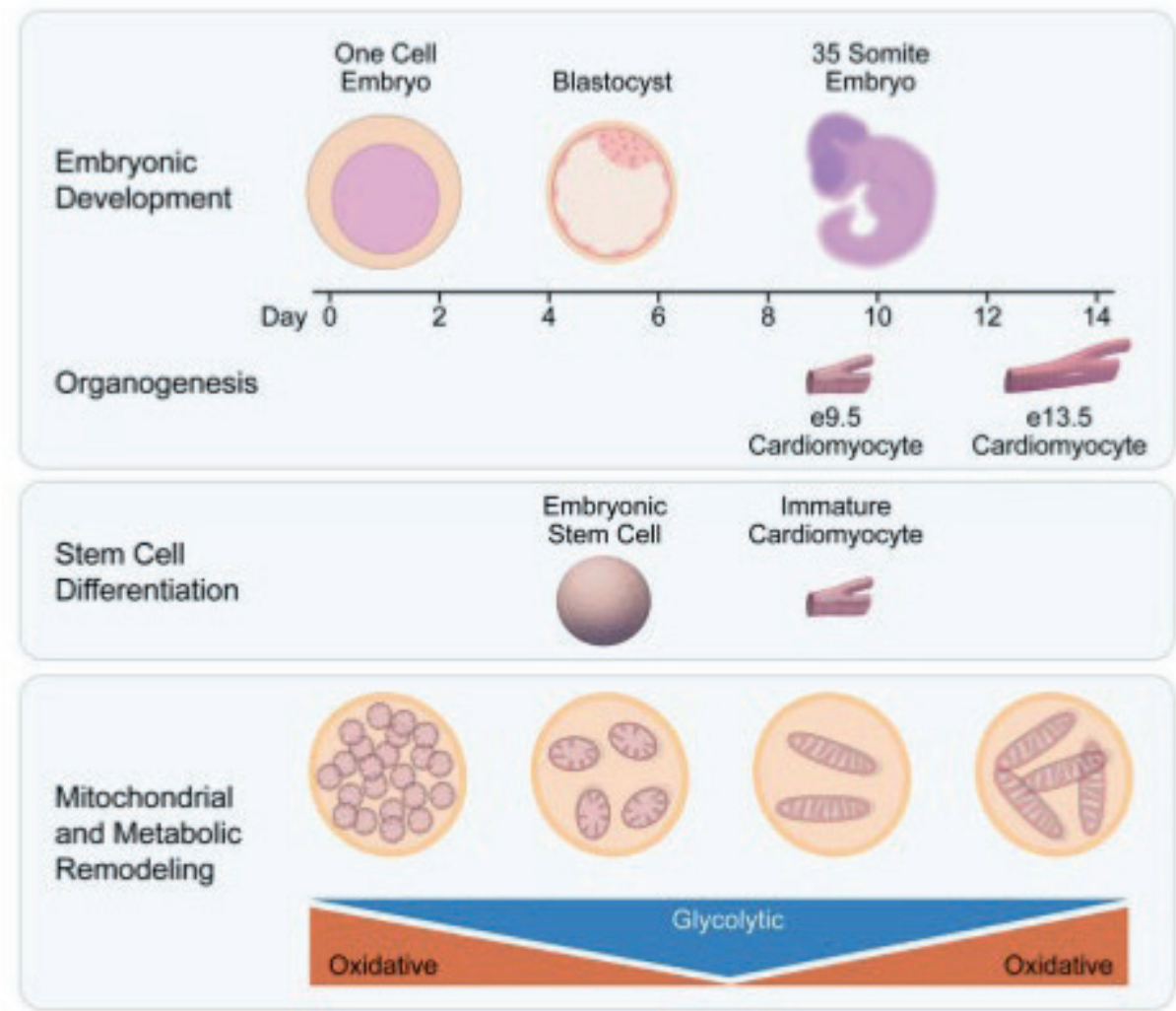

Figure 5. Energy metabolism fuels developmental organogenesis and stem cell differentiation. Developmental organogenesis reflects an ongoing metamorphosis in energy metabolism underscoring continues interplay of infrastructural inherited metabolism and evolve energy production as adapted from Folmes et al in 2014. Metabolic determinants of embryonic development and stem cell fate. Reproduction Fertility Development. 2014 December; 27(1): 82-88.doi:10.1071/RD14383.

All these examples show the importance of controlling mtDNA copy number and metabolism for healthy development and combating disease. Insight in the mechanisms may lead to better risk prediction for mtDNA mutation carriers, to interventions for reducing mtDNA mutation load, or to higher pregnancy rates for IVF, and maybe treatment options for cancer. 


\section{References}

1. Bernier, F.P., et al., Diagnostic criteria for respiratory chain disorders in adults and children. Neurology, 2002. 59(9): p. 1406-11.

2. Witters, P., et al., Revisiting mitochondrial diagnostic criteria in the new era of genomics. Genet Med, 2018. 20(4): p. 444-451.

3. Theunissen, T.E.J., et al., Whole Exome Sequencing Is the Preferred Strategy to Identify the Genetic Defect in Patients With a Probable or Possible Mitochondrial Cause. Front Genet, 2018. 9: p. 400.

4. Kirby, D.M. and D.R. Thorburn, Approaches to finding the molecular basis of mitochondrial oxidative phosphorylation disorders. Twin Res Hum Genet, 2008. 11(4): p. 395-411.

5. Yang, Y., et al., Clinical whole-exome sequencing for the diagnosis of mendelian disorders. N Engl J Med, 2013. 369(16): p. 1502-11.

6. Lieber, D.S., et al., Targeted exome sequencing of suspected mitochondrial disorders. Neurology, 2013. 80(19): p. 1762-70.

7. Calvo, S.E., et al., Molecular Diagnosis of Infantile Mitochondrial Disease with Targeted Next-Generation Sequencing. Science Translational Medicine, 2012. 4(118).

8. Wortmann, S.B., et al., Mutations in the phospholipid remodeling gene SERAC1 impair mitochondrial function and intracellular cholesterol trafficking and cause dystonia and deafness. Nat Genet, 2012. 44(7): p. 797-802.

9. Pronicka, E., et al., New perspective in diagnostics of mitochondrial disorders: two years' experience with whole-exome sequencing at a national paediatric centre. J Transl Med, 2016. 14(1): p. 174.

10. Theunissen, T.E.J., et al., Rapid Resolution of Blended or Composite Multigenic Disease in Infants by Whole-Exome Sequencing. J Pediatr, 2017. 182: p. 371-374 e2.

11. Kamps, R., et al., Genetic defects in mtDNA-encoded protein translation cause pediatric, mitochondrial cardiomyopathy with early-onset brain disease. Eur J Hum Genet, 2018. 26(4): p. 537-551.

12. Kohda, M., et al., A Comprehensive Genomic Analysis Reveals the Genetic Landscape of Mitochondrial Respiratory Chain Complex Deficiencies. PLoS Genet, 2016. 12(1): p. e1005679.

13. Dallabona, C., et al., Novel (ovario) leukodystrophy related to AARS2 mutations. Neurology, 2014. 82(23): p. 2063-71.

14. Euro, L., et al., Structural modeling of tissue-specific mitochondrial alanyltRNA synthetase (AARS2) defects predicts differential effects on aminoacylation. Front Genet, 2015. 6: p. 21.

15. Dimauro, S. and G. Davidzon, Mitochondrial DNA and disease. Ann Med, 2005. 37(3): p. 222-32.

16. Jiang, P., et al., Biochemical evidence for a mitochondrial genetic modifier in the phenotypic manifestation of Leber's hereditary optic neuropathyassociated mitochondrial DNA mutation. Hum Mol Genet, 2016. 25(16): p. 3613-3625. 
17. Spruijt, L., et al., Influence of mutation type on clinical expression of Leber hereditary optic neuropathy. Am J Ophthalmol, 2006. 141(4): p. 676-82.

18. Schulze, T.G., et al., Molecular genetic overlap in bipolar disorder, schizophrenia, and major depressive disorder. World J Biol Psychiatry, 2014. 15(3): p. 200-8.

19. Billingsley, K.J., et al., Mitochondria function associated genes contribute to Parkinson's Disease risk and later age at onset. NPJ Parkinsons Dis, 2019. 5: p. 8.

20. Sallevelt, S., et al., A comprehensive strategy for exome-based preconception carrier screening. Genet Med, 2017. 19(5): p. 583-592.

21. Cragun, D., et al., Panel-based testing for inherited colorectal cancer: a descriptive study of clinical testing performed by a US laboratory. Clin Genet, 2014. 86(6): p. 510-20.

22. Yurgelun, M.B., et al., Identification of a Variety of Mutations in Cancer Predisposition Genes in Patients With Suspected Lynch Syndrome. Gastroenterology, 2015. 149(3): p. 604-13 e20.

23. Desmond, A., et al., Clinical Actionability of Multigene Panel Testing for Hereditary Breast and Ovarian Cancer Risk Assessment. JAMA Oncol, 2015. 1(7): p. 943-51.

24. Frey, M.K., et al., Rescreening for genetic mutations using multi-gene panel testing in patients who previously underwent non-informative genetic screening. Gynecol Oncol, 2015. 139(2): p. 211-5.

25. Mortazavi, A., et al., Mapping and quantifying mammalian transcriptomes by RNA-Seq. Nat Methods, 2008. 5(7): p. 621-8.

26. Sultan, M., et al., A global view of gene activity and alternative splicing by deep sequencing of the human transcriptome. Science, 2008. 321(5891): p. 956-60.

27. Morin, R., et al., Profiling the HeLa 53 transcriptome using randomly primed CDNA and massively parallel short-read sequencing. Biotechniques, 2008. 45(1): p. 81-94.

28. Ramaswami, G., et al., Accurate identification of human Alu and non-Alu RNA editing sites. Nat Methods, 2012. 9(6): p. 579-81.

29. Cummings, B.B., et al., Improving genetic diagnosis in Mendelian disease with transcriptome sequencing. bioRxiv, 2016.

30. Kent, W.J., et al., The human genome browser at UCSC. Genome Res, 2002. 12(6): p. 996-1006.

31. Gonzaga-Jauregui, C., J.R. Lupski, and R.A. Gibbs, Human genome sequencing in health and disease. Annu Rev Med, 2012. 63: p. 35-61.

32. Ekblom, R. and J.B. Wolf, A field guide to whole-genome sequencing, assembly and annotation. Evol Appl, 2014. 7(9): p. 1026-42.

33. Miao, H., et al., Long-read sequencing identified a causal structural variant in an exome-negative case and enabled preimplantation genetic diagnosis. Hereditas, 2018. 155: p. 32.

34. Cacace, R., et al., Loss of DPP6 in neurodegenerative dementia: a genetic player in the dysfunction of neuronal excitability. Acta Neuropathol, 2019. 137(6): p. 901-918. 
35. Clark, M.M., et al., Diagnosis of genetic diseases in seriously ill children by rapid whole-genome sequencing and automated phenotyping and interpretation. Sci Transl Med, 2019. 11(489).

36. ZonMw. WGS First. 2019, 20 September; Available from: https://www.wgs-first.nl/en/.

37. International Cancer Genome, C., et al., International network of cancer genome projects. Nature, 2010. 464(7291): p. 993-8.

38. Cancer Genome Atlas Research, N., et al., The Cancer Genome Atlas PanCancer analysis project. Nat Genet, 2013. 45(10): p. 1113-20.

39. Turnbull, C., Introducing whole-genome sequencing into routine cancer care: the Genomics England 100000 Genomes Project. Ann Oncol, 2018. 29(4): p. 784-787.

40. News, A.P.S.N.E.G.E.B. The 100,000 Genomes Club. 2019, 20 September; Available from: https://www.genengnews.com/insights/the100000-genomes-club/.

41. Francioli, L.C., et al., Whole-genome sequence variation, population structure and demographic history of the Dutch population. Nature Genetics, 2014. 46(8): p. 818-825.

42. Lappalainen, T., et al., Genomic Analysis in the Age of Human Genome Sequencing. Cell, 2019. 177(1): p. 70-84.

43. OMIM, OMIM. August 23, 2019.

44. Fokkema, I.F., et al., LOVD v.2.0: the next generation in gene variant databases. Hum Mutat, 2011. 32(5): p. 557-63.

45. ClinVar. ClinVar. 2019, 6 August; Available from: https://www.ncbi.nlm.nih.gov/clinvar/.

46. Alamut, Alamut. August 23, 2019.

47. PolyPhen-2, PolyPhen. August 23, 2019.

48. SIFT, SIFT. August 23, 2019.

49. Wallis, Y.P., S; McAnulty, C; Bodmer, D; Sister-man, E; Robertson, K; Moore, D; Abbs, S; Deans, Z; Devereau, A., Practice Guidelines for the Evaluation of Pathogenicity and the Reporting of Sequence Variants in Clinical Molecular Genetics. 2013, Birmingham, UK: Association for Clinical Genetic Science.

50. DiMauro, S. and E.A. Schon, Mitochondrial DNA mutations in human disease. Am J Med Genet, 2001. 106(1): p. 18-26.

51. McFarland, R., et al., Assigning pathogenicity to mitochondrial tRNA mutations: when "definitely maybe" is not good enough. Trends Genet, 2004. 20(12): p. 591-6.

52. Yarham, J.W., et al., A comparative analysis approach to determining the pathogenicity of mitochondrial tRNA mutations. Hum Mutat, 2011. 32(11): p. 1319-25.

53. Gonzalez-Vioque, E., et al., The pathogenicity scoring system for mitochondrial tRNA mutations revisited. Mol Genet Genomic Med, 2014. 2(2): p. 107-14. 
54. Ruiz-Pesini, E., et al., An enhanced MITOMAP with a global mtDNA mutational phylogeny. Nucleic Acids Res, 2007. 35(Database issue): p. D823-8.

55. Putz, J., et al., Mamit-tRNA, a database of mammalian mitochondrial tRNA primary and secondary structures. RNA, 2007. 13(8): p. 1184-90.

56. Bris, C., et al., Bioinformatics Tools and Databases to Assess the Pathogenicity of Mitochondrial DNA Variants in the Field of Next Generation Sequencing. Front Genet, 2018. 9: p. 632.

57. Wilkins, H.M., S.M. Carl, and R.H. Swerdlow, Cytoplasmic hybrid (cybrid) cell lines as a practical model for mitochondriopathies. Redox Biology, 2014. 2: p. 619-631.

58. Kremer, L.S., S.B. Wortmann, and H. Prokisch, "Transcriptomics": molecular diagnosis of inborn errors of metabolism via RNA-sequencing. J Inherit Metab Dis, 2018. 41(3): p. 525-532.

59. Calvo, S.E. and V.K. Mootha, The mitochondrial proteome and human disease. Annu Rev Genomics Hum Genet, 2010. 11: p. 25-44.

60. Abela, L., et al., N(8)-acetylspermidine as a potential plasma biomarker for Snyder-Robinson syndrome identified by clinical metabolomics. J Inherit Metab Dis, 2016. 39(1): p. 131-7.

61. McCormick, E.M., Z. Zolkipli-Cunningham, and M.J. Falk, Mitochondrial disease genetics update: recent insights into the molecular diagnosis and expanding phenotype of primary mitochondrial disease. Current Opinion in Pediatrics, 2018. 30(6): p. 714-724.

62. Muraresku, C.C., E.M. McCormick, and M.J. Falk, Mitochondrial Disease: Advances in clinical diagnosis, management, therapeutic development, and preventative strategies. Curr Genet Med Rep, 2018. 6(2): p. 62-72.

63. Davis, R.L., et al., Fibroblast growth factor 21 is a sensitive biomarker of mitochondrial disease. Neurology, 2013. 81(21): p. 1819-26.

64. Suomalainen, A., et al., FGF-21 as a biomarker for muscle-manifesting mitochondrial respiratory chain deficiencies: a diagnostic study. Lancet Neurol, 2011. 10(9): p. 806-18.

65. Liang, C., K. Ahmad, and C.M. Sue, The broadening spectrum of mitochondrial disease: shifts in the diagnostic paradigm. Biochim Biophys Acta, 2014. 1840(4): p. 1360-7.

66. Gerards, M., et al., Riboflavin-responsive oxidative phosphorylation complex I deficiency caused by defective ACAD9: new function for an old gene. Brain, 2011. 134(Pt 1): p. 210-9.

67. Repp, B.M., et al., Clinical, biochemical and genetic spectrum of 70 patients with ACAD9 deficiency: is riboflavin supplementation effective? Orphanet J Rare Dis, 2018. 13(1): p. 120.

68. Haack, T.B., et al., Exome sequencing identifies ACAD9 mutations as a cause of complex I deficiency. Nat Genet, 2010. 42(12): p. 1131-4.

69. Theunissen, T.E.J., et al., Selection and Characterization of Palmitic Acid Responsive Patients with an OXPHOS Complex I Defect. Front Mol Neurosci, 2017. 10: p. 336. 
70. Gerards, M., et al., Exome sequencing reveals a novel Moroccan founder mutation in SLC19A3 as a new cause of early-childhood fatal Leigh syndrome. Brain, 2013. 136(Pt 3): p. 882-90.

71. Gerards, M., Leigh syndrome: the genetic heterogeneity story continues. Brain, 2014. 137(Pt 11): p. 2872-3.

72. Gerards, M., S.C. Sallevelt, and H.J. Smeets, Leigh syndrome: Resolving the clinical and genetic heterogeneity paves the way for treatment options. Mol Genet Metab, 2016. 117(3): p. 300-12.

73. Otten, A.B. and H.J. Smeets, Evolutionary defined role of the mitochondrial DNA in fertility, disease and ageing. Hum Reprod Update, 2015. 21(5): p. 671-89.

74. Sallevelt, S., Dreesen JCFM, de Coo, IFM et al., Selecting the right embryo in mitochondrial disorders, ed. C. 17. Vol. Chapter 17:231-246. 2015: Molecular Genetics in Reproductive Medicine.

75. Sallevelt, S.C.E.H., et al., Preimplantation genetic diagnosis in mitochondrial DNA disorders: challenge and success. Journal of Medical Genetics, 2013. 50(2): p. 125-132.

76. https://www.umdf.org/mitochondrial-replacement-therapy/Accessed on July 3, 2020

77. Otten, A.B.C., et al., Mutation-specific effects in germline transmission of pathogenic mtDNA variants. Hum Reprod, 2018. 33(7): p. 1331-1341.

78. Hyslop, L.A., et al., Towards clinical application of pronuclear transfer to prevent mitochondrial DNA disease. Nature, 2016. 534(7607): p. 383-6.

79. Hyslop, L.A., et al., Corrigendum: Towards clinical application of pronuclear transfer to prevent mitochondrial DNA disease. Nature, 2016. 538(7626): p. 542.

80. Keeling, P.J., et al., The tree of eukaryotes. Trends Ecol Evol, 2005. 20(12): p. 670-6.

81. Jenuth, J.P., et al., Random genetic drift in the female germline explains the rapid segregation of mammalian mitochondrial DNA. Nat Genet, 1996. 14(2): p. 146-51.

82. Brown, D.T., et al., Random genetic drift determines the level of mutant mtDNA in human primary oocytes. Am J Hum Genet, 2001. 68(2): p. 5336.

83. Wilson, I.J., et al., Mitochondrial DNA sequence characteristics modulate the size of the genetic bottleneck. Hum Mol Genet, 2016. 25(5): p. 103141.

84. Stewart, J.B. and P.F. Chinnery, The dynamics of mitochondrial DNA heteroplasmy: implications for human health and disease. Nat Rev Genet, 2015. 16(9): p. 530-42.

85. Freyer, C., et al., Variation in germline mtDNA heteroplasmy is determined prenatally but modified during subsequent transmission. Nat Genet, 2012. 44(11): p. 1282-5.

86. Trifunovic, A., et al., Premature ageing in mice expressing defective mitochondrial DNA polymerase. Nature, 2004. 429(6990): p. 417-23. 
87. Olivo, P.D., et al., Nucleotide sequence evidence for rapid genotypic shifts in the bovine mitochondrial DNA D-loop. Nature, 1983. 306(5941): p. 400-2.

88. Lane, N. and W. Martin. The energetics of genome complexity. Nature 2010 Oct 21 [cited 467 7318]; 2010/10/22:[929-34]. Available from: https://www.ncbi.nlm.nih.gov/pubmed/20962839.

89. Cree, L.M., et al., A reduction of mitochondrial DNA molecules during embryogenesis explains the rapid segregation of genotypes. Nat Genet, 2008. 40(2): p. 249-54.

90. Wai, T., D. Teoli, and E.A. Shoubridge, The mitochondrial DNA genetic bottleneck results from replication of a subpopulation of genomes. Nature Genetics, 2008. 40(12): p. 1484-1488.

91. Carling, P.J., L.M. Cree, and P.F. Chinnery, The implications of mitochondrial DNA copy number regulation during embryogenesis. Mitochondrion, 2011. 11(5): p. 686-92.

92. Fan, W., et al., A mouse model of mitochondrial disease reveals germline selection against severe mtDNA mutations. Science, 2008. 319(5865): p. 958-62.

93. Shutt, T.E., M. Bestwick, and G.S. Shadel, The core human mitochondrial transcription initiation complex: It only takes two to tango. Transcription, 2011. 2(2): p. 55-59.

94. Ylikallio, E., et al., High mitochondrial DNA copy number has detrimental effects in mice. Hum Mol Genet, 2010. 19(13): p. 2695-705.

95. Folmes, C.D. and A. Terzic, Metabolic determinants of embryonic development and stem cell fate. Reprod Fertil Dev, 2014. 27(1): p. 82-8.

96. Otten, A.B., et al., Differences in Strength and Timing of the mtDNA Bottleneck between Zebrafish Germline and Non-germline Cells. Cell Rep, 2016. 16(3): p. 622-30.

97. St John, J., The control of mtDNA replication during differentiation and development. Biochim Biophys Acta, 2014. 1840(4): p. 1345-54.

98. Payne, B.A., et al., Mitochondrial aging is accelerated by anti-retroviral therapy through the clonal expansion of mtDNA mutations. Nat Genet, 2011. 43(8): p. 806-10.

99. Cecchino, G.N. and J.A. Garcia-Velasco, Mitochondrial DNA copy number as a predictor of embryo viability. Fertil Steril, 2019. 111(2): p. 205-211.

100. Fragouli, E., et al., Altered levels of mitochondrial DNA are associated with female age, aneuploidy, and provide an independent measure of embryonic implantation potential. PLoS Genet, 2015. 11(6): p. e1005241.

101. Viotti, M., et al., Is mitochondrial DNA quantitation in blastocyst trophectoderm cells predictive of developmental competence and outcome in clinical IVF? J Assist Reprod Genet, 2017. 34(12): p. 15811585.

102. Victor, A.R., et al., Accurate quantitation of mitochondrial DNA reveals uniform levels in human blastocysts irrespective of ploidy, age, or implantation potential. Fertil Steril, 2017. 107(1): p. 34-42 e3. 
Chapter 7

103. Diez-Juan, A., et al., Mitochondrial DNA content as a viability score in human euploid embryos: less is better. Fertil Steril, 2015. 104(3): p. 53441 e1. 
Summary 
Mitochondrial diseases result from a reduced energy production capacity in the mitochondria due to deficiencies in oxidative phosphorylation (OXPHOS). They are characterized by clinically highly heterogeneous and tissue-specific manifestations, typically involving tissues and organs with high energy requirements, like brain, heart and muscle. As the OXPHOS system is under dual genetic control, mutations in both the mitochondrial DNA and nuclear DNA can lead to a mitochondrial disease (Chapter 1). Mainly as a consequence of the large genetic heterogeneity (possibly more than 1500 genes involved) and clinical variability, for the majority of patients a genetic cause was unknown or not feasible at the start of the project. So, the first aim of the work described in this thesis was to identify and functionally characterize the underlying genetic cause in either the mtDNA or in nuclear genes in patients with OXPHOS disease. Based on a genetic diagnosis, counselling can be offered to the family and prenatal or preimplantation diagnosis to prevent the transmission of the mitochondrial disorder to further offspring. In case of an mtDNA disease this is less straightforward than for nuclear gene defects, as a mitochondrial bottleneck occurs during oogenesis, defining the mutation load and disease risk in the offspring. Although crucial for understanding the role of mitochondria in oogenesis, embryogenesis and the transmission of mtDNA disease, the mitochondrial bottleneck is still poorly understood, also because of the lack of appropriate animal models. The second aim our work was to develop an animal model for studying and manipulating the mitochondrial bottleneck.

In Chapter 2 we described the use of next-generation sequencing (NGS) technology and discussed the impact it has on identifying pathogenic variants causing genetic disease, or on identifying genetic risk factors. The potential and limitations of different technologies and diagnostic approaches (i.e. targeted NGS, whole-exome sequencing (WES), RNA-Seq and whole genome sequencing (WGS)) are discussed. NGS technology expanded rapidly in the last decades with significant improvements in reliability, sequencing chemistry, pipeline analyses, data interpretation and costs. Such advances made the use of NGS the preferred choice today not only in clinical genetics practice. 
A number of clinical applications of NGS have been addressed, like detection of the causative mutation in inherited cancer syndromes by DNA-sequencing and of splice variants by RNA-sequencing. Applications for pre-implantation genetic diagnosis, cancer somatic mutation analysis, pharmacogenetics, using blood DNA or liquid biopsy were discussed. Clinical limitations and ethical considerations related to the use of NGS for these applications are mentioned. Although this chapter reviews more general clinical application of NGS, it is evident that the topics addressed are relevant for the neurological and mitochondrial diseases, which are the prime topic of this thesis.

NGS is successfully used to determine the primary underlying genetic defect in clinically highly heterogeneous inherited mitochondrial diseases which has facilitated a rapid introduction in diagnostics and clinical care of these and other diseases, as described in Chapter 2. WES as first strategy includes all variants in the coding part of the genome, allowing also the identification of risk factors, which can increase the risk for a disease by themselves or in combination with a primary genetic defect or with other risk factors. It is likely that genetic risk factors are involved in mitochondrial disease, illustrated by the highly variable manifestation of the m.3243A>G mutation or the male preponderance in LHON. Also, in a substantial part of the patients with mitochondrial diseases, no primary genetic cause has been identified, which means that it is either missed by WES or that the disease is not caused by a single primary genetic defect. A combination of multiple genetic risk factors, defined by a polygenic risk score could provide an alternative explanation, as has been demonstrated for example in the cancer field. These polygenic scores have been calculated by summing-up genetic risks associated with known SNPs in GWAS data. In Chapter $\mathbf{3}$ we describe as a proof of concept the identification and contribution of genetic risk factors in candidate genes to the severity of clinical manifestation in Lynch syndrome. A similar approach could be applied to identify genetic factors explaining the clinical heterogeneity of in mtDNA or nuclear mitochondrial genes.

In Chapter 3 we used targeted NGS to detect co-occurring rare variants as possible genetic modifiers in Lynch syndrome by analysing a panel of candidate genes in 35 Lynch syndrome mutation carriers with a poor clinical phenotype. The 
gene panel consisted of 154 genes involved in endometrial physiology and carcinogenesis. Data analysis was done by using a simple pipeline, in which variants were selected based on an allele frequency lower than 0.001 and on predicted non-conservative amino-acid substitutions. A total of 54 variants (categories 3 = VUS, or 4 = likely pathogenic, or 5 = pathogenic) were considered as putative risk modifiers. Risk prediction based on these variants was difficult to validate, but we proposed potential risk-variants and the predicted protein change for 6 functional known genes (RET; p.(Asn361Lys), CYP1A1; p.(Cys457*), IGF1R;p.(Thr758Met), NCOR2;p.(Ser1964Cys), COMT; p.(Lys159del), mTOR; p.(Met1313Leu). Logistic regression analysis, including two or more risk modifying variants in women carrying a primary pathogenic mutation, was associated with a poor clinical phenotype. A similar strategy could be performed to characterize genetic modifiers in mitochondrial disease. Further, in silico modeling, and in vitro, and in vivo studies will be needed to explore the impact of these variants on protein function and cellular OXPHOS processes. The role of genetic modifiers in mitochondrial disease patients will be relevant to explain the group without a genetic diagnosis or as a contributing factor in heterogeneous disease manifestation and could elucidate potential treatment options in future.

A search for genetic modifiers, will complement the search for primary genetic causes in families with mitochondrial diseases, as described in Chapter $\mathbf{4}$ and $\mathbf{5}$. The diagnosis was based on clinical symptoms, including MRI and deficiencies in OXPHOS complexes in muscle or fibroblasts of patients. Evidence for the pathogenicity of novel variants and novel genes was provided by complementation assays. Leigh syndrome (Chapter 4 ) is an early onset, often fatal progressive neurodegenerative disorder caused by mutations in the mitochondrial or nuclear DNA. Until now, mutations in more than 60 genes had been reported to cause Leigh syndrome, indicating an extreme genetic heterogeneity for this disorder, but still only explaining part of the cases. WES was combined with homozygosity mapping as an additional filtering step to identify the underlying genetic defect in a Moroccan family with fatal Leigh syndrome in early childhood and specific MRI abnormalities in the brain. We detected a homozygous nonsense mutation (c.20C>A; p.Ser7Ter) in the thiamine transporter SLC19A3. In vivo overexpression of wild-type SLC19A3 showed an increased thiamine uptake, 
whereas overexpression of mutant SLC19A3 did not, confirming that the mutations resulted in an absent or non-functional transporter. Seventeen additional patients with LS were screened for mutations in SLC19A3 using conventional Sanger sequencing. Two unrelated patients both Moroccan origin and one from consanguineous parents were homozygous for the same p.Ser7Ter mutation. One of these patients showed the same MRI abnormalities as the patients from the first family. Strikingly, patients who received thiamine had an improved life-expectancy. One patient in the third family deteriorated upon interruption of the thiamine treatment and recovered after reinitiating. Although unrelated, all patients came from the province Al Hoceima in Northern Morocco. Based on the recombination events the foundermutation was estimated to have occurred 1250-1750 years ago. Our data showed that the SLC19A3 gene is a new candidate for mutation screening in patients with Leigh syndrome, who might benefit from high doses of thiamine. Especially Moroccan Leigh patients should be tested for the c.20C>A founder mutation in SLC19A3.

In Chapter 5, we describe the application of whole-exome sequencing in three patients with paediatric cardiomyopathy and early-onset brain disease with OXPHOS deficiencies. The brain pathology was studied by MRI analysis. In consanguineous patient 1 we identified a homozygous intronic variant (c.850$3 A>G)$ in the QRSL1 gene, which was predicted to cause abnormal splicing. The variant segregated with the disease and affected the protein function, which was confirmed by complementation studies, restoring OXPHOS function only with wildtype QRSL1. Patient 2 was compound heterozygous for two novel disease causing variants (c. [253G >A]; [938G >A]) in the MTO1 gene. In patient 3 we detected one unknown disease-causing variant (c.2872C $>$ T) and one known disease-causing variant (c. $1774 \mathrm{C}>\mathrm{T}$ ) in the $A A R S 2$ gene. The $\mathrm{c} .1774 \mathrm{C}>\mathrm{T}$ variant was present in the paternal copy of the AARS2 gene, the c.2872C $>\mathrm{T}$ in the maternal copy. All genes were involved in translation of mtDNA-encoded proteins. Defects in mtDNAencoded protein translation lead to severe paediatric cardiomyopathy and brain disease with OXPHOS abnormalities. This suggests that the heart and brain are particularly sensitive to defects in mitochondrial protein synthesis during late embryonic or early postnatal development, probably due to the massive 
mitochondrial biogenesis occurring at that stage. If both the heart and brain are involved, the prognosis is poor with a likely fatal outcome at young age.

In the families, described in Chapter $\mathbf{4}$ and 5, but also in many others, as mentioned in Chapter 2, we demonstrated the importance of NGS and WES in genetic testing for mitochondrial disease, increasing the diagnostic yield from $25 \%$ to $69 \%$ and a turn-around time from years to months. Furthermore, we identified in $30 \%$ of the cases defects in novel genes, indicating that unbiased Whole Exome Sequencing combined with RNA-Seq is still superior to panel-based approaches in mitochondrial diseases.

Our second aim was to develop an animal model for studying and manipulating the mitochondrial bottleneck. We have shown before that zebrafish is an excellent model, as both timing and size of the bottleneck are highly conserved from fish to man. Zebrafish provide sufficient numbers of offspring and are easy to manipulate. Both the embryonic development and oogenesis can be studied in full detail. To understand the pathology and mechanisms associated with low mtDNA copy numbers, we manipulated the mtDNA bottleneck by knocking down Tfam, a key regulator of mtDNA replication, during early zebrafish development in Chapter 6. Reduction of Tfam using a splice-MO, resulted in a $42 \% \pm 17 \%$ decrease in the mtDNA copy number in zebrafish embryos at 4 days post fertilization (dpf). Morphant embryos displayed abnormal development of the eye, brain, heart and muscle, as well as a $50 \% \pm 22 \%$ decrease in ATP production. Transcriptome analysis revealed a decrease in protein-encoding transcripts from the heavy strand of the mtDNA, and down-regulation of genes involved in haem production and the metabolism of metabolites, which appear to trigger increased rRNA and tRNA synthesis in the nucleoli. However, this stress or compensatory response appears to fall short as pathology emerges and expression of genes related to eye development are severely down-regulated. Taken together, this study highlights the importance of sufficient mtDNA copies for early zebrafish development. Zebrafish is an excellent model to manipulate the mtDNA bottleneck and study its effect on embryogenesis rapidly and in large numbers of offspring. 
In Chapter 7, we discussed the impact of NGS not only the diagnosis of mitochondrial disease, but also for personalized medicine in general. It is beyond any doubt, that WGS in combination with RNA-Seq will bring the paradigm shift started by WES to the diagnostic next level. There will be limited need to narrow down the differential clinical diagnosis to request sequencing of a specific gene or sets of genes, as all genetic information will be available at once from start. The results of this genetic analysis will directly guide the diagnostic patient evaluation, moving towards personalized patient care and treatment in mitochondrial diseases. Complete analysis of both the mtDNA and exome generated a diagnostic yield of $69 \%$, obtained in months, which by far exceeds the $25 \%$ yield using conventional, targeted sequencing. As indicated before, this means there is still an era for improvement in unravelling the genetic cause of the undiagnosed cases using WGS, RNA-Seq or polygenic risk scores in these unsolved mitochondrial patients.

Furthermore, as we succeeded in the development of the first tuneable zebrafish model to study the mtDNA bottleneck in embryogenesis, we discuss on the impact this will have on characterizing and manipulating the mitochondrial bottleneck and the processes triggered by a reduced mtDNA copy number in zebrafish embryos in further detail. If we will be able to generate mtDNA mutations in those zebrafish, then this will become an excellent model for testing the current three different mitochondrial bottleneck theories, for identifying possible selection mechanisms and for risk assessment of mtDNA disease transmission. 


\section{CHAPTER 8}

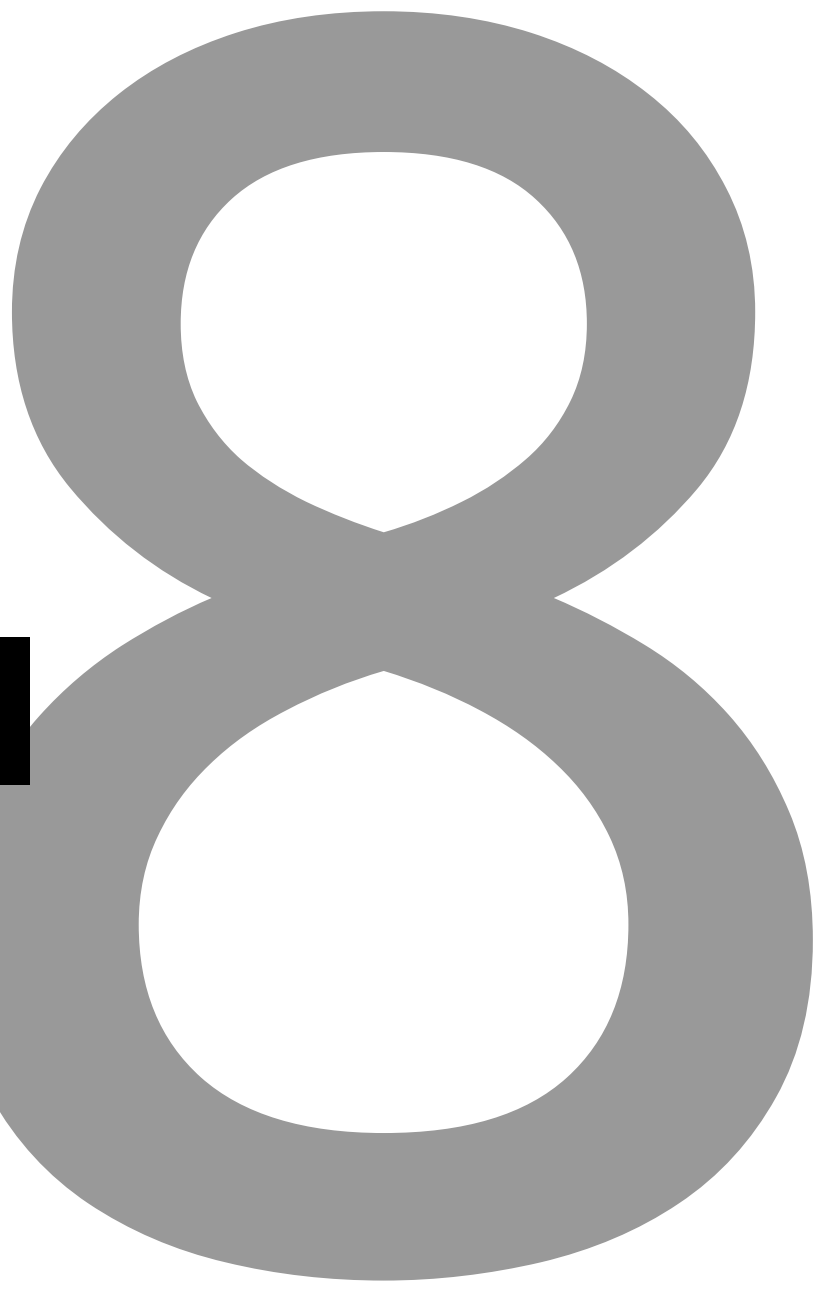


Valorisation 
Mitochondrial diseases are the most common genetic metabolic diseases, affecting approximately 1 in 5,000 individuals [1]. Mitochondrial diseases are a clinically and genetically heterogeneous group of disorders, which generally manifest in tissues or organs with a high-energy requirement [2]. Due to this complexity and the lack of proper genotype-phenotype correlations, at the start of the work performed in this thesis, a genetic diagnosis was obtained in only $25 \%$ of patients by sequential analysis of the mtDNA and single candidate genes. The application of next-generation sequencing as a research tool to first sequence gene panels and later all genes (the exome) has increased this figure considerably, which has facilitated a rapid introduction in diagnostics and clinical care [3]. The valorisation paragraph will deal with the introduction of NGS in routine genetic diagnostics.

Next-generation sequencing: from research to diagnostics

A genetic diagnosis is important for counseling patient and relatives, for preventing the transmission, for providing a more accurate prognosis and in some cases for offering therapeutic interventions. During the past years, NGS technology has developed as the preferred tool for identifying the genetic defects in patients with clinically and genetically heterogenous diseases (Chapter 2). NGS has contributed in identifying the genetic cause in either the mtDNA (20\%) or nDNA (WES; 49\%), leading to an overall diagnostic yield of $69 \%$ [4]. Given the impressive increase not only in the percentage of patients, in which a genetic diagnosis is established (from $25 \%$ to $69 \%$ ), but also in the speed (from years to months) and in costs (expenisve Sanger Sequencing), a rapid introduction of NGS in routine genetic diagnosis of mitochondrial disease was warranted and has been established in the last couple of years. NGS costs rapidly declined and the capacity of platforms as the Illumina NovaSeq 6000 increased tremendously. For example 200 exomes can be sequenced in a single sequencing run. Crucial in this respect was also the development of novel improved bioinformatics tools to predict variant pathogenicity and the availability of superior databases with information on exomes (Exac) and genetic variants (ClinVar). The adagium will be NGS first and guided by the genes and variants found, specific clinical and laboratory investigations can be carried out to unambiguously link the genetic $\operatorname{defect}(\mathrm{s})$ to the clinical manifestations. The laboratory findings may also be important, in case 
functional validation is required. Our results show a few key points for proper introduction into the clinic:

\section{NGS of both the mtDNA and the whole exome}

It is essential investigate both the mtDNA and the exome to identify the genetic cause in mitochondrial disease [4-6]. Although the mtDNA can be analysed in the exome DNA, the coverage is insufficient to detect the necessary low level mutation loads in blood DNA, which might indicate a higher mutation load and follow-up in affected tissues. NGS of the mtDNA allows detection of point mutations and rearragements alike with immediate characterization of the breakpoints. Only the mtDNA copy number has to be established by a separate test.

2. Novel mitochondrial disease genes

Novel genes are still being identified as a possible cause of mitochondrial disease. These are either genes, in which no genetic defect has been found so far, or genes, in which defects have been found in apparently nonmitochondrial disease. This implies that gene panel-based approaches fall short, as the mitochondrial panel is still not complete. Complete analysis of all genes in the exome (open exome) [4] is preferable to start with to identify all relevant genetic defects in the shortest timeframe and at the lowest cost.

3. Overlap between mitochondrial and other neuromuscular disorders

Our results demonstrate that many neuromuscular diseases exist, in which a mitochondrial defect can be the cause in the minority of cases. In case of gene panels, this would imply sequential analysis of multiple, and probably partly overlapping gene panels. Again, it is preferable to start with an open exome [4], as this would allow an unbiased identification of the underlying genetic causes.

4. De novo mutations

As we detected de novo mutations in the clinical exome, it would be preferable to include immediately the parents for whole exome sequencing [7] and analyze the data, using an autosomal recessive and de novo dominant disease model.

\section{Multigenic disease}

Especially in clinically heterogeneous disease and when consanguinity is involved, the option of having multiple genes causing the disease in the 
patient, should be investigated. This can be done by investigating all gene defects, which could be potentially contribute to the clinical phenotype. One should not address all symptoms to the first gene identifed. As this has been done in the past, it is clear that genotype-phenotype correlations from literature [8], when based on few patients and single gene analysis, should be considered with care.

Our proposed diagnostic strategy for most mitochondrial diseases will be NGS of the mtDNA, followed by whole exome sequencing of patients and parents, followed by analysis of all genes, using an autosomal recessive or de novo dominant genetic model. In case of new genes or unclassified variants, functional follow-up sudies should be offered to demonstrate pathogenicity. In case an OXPHOS deficiency is observed in patient-derived fibroblasts, this can be done straightforward by complementation assays. If this is not the case, animal studies might provide the answer, which will often go beyond the capabilities of routine diagnostic labs. An in vivo model as zebrafish could fill this gap, but standardization, validation and high-throughput processing is required to keep-up with the rapid generation of unclassified variants and novel genes by WES and WGS these days.

As the combined approach will identifiy at max $69 \%$ of the genetic causes, still $31 \%$ of the patients will remain without a genetic diagnosis. As these are carefully selcted patients from our cohort, it is likely that that there will be technical issues, prohibiting the identification of the underlying defects. Applying WGS could solve the issue, but routine use is still limited by the financial costs and the complexity of data interpretation. Rapid and life-saving genetic diagnosis in less than 24 hours using rapid WGS (rWGS) is possible and already offered for early intervention cases within paediatric Intensive Care Units (ICUs) as discussed in Chapter 7. Introducing rWGS showed a diagnostic yield of 42 to $57 \%$ in serious ill infants in ICUs, and changes in medical management in 30 to $72 \%$, and finally altered outcomes in 24 to $34 \%$ of the cases [9]. Sequencing the transcriptome by RNASeq seems at this moment a more realistic addition to WES to solve the remaining cases. RNA-Seq allowed analysis of the transcriptome at an unprecedented depth and reported the identification of causative, non-exonic regulatory variants in an additional $10 \%$ diagnostic yield of mitochondrial patients using RNA-Seq [10]. In another study of rare muscular disorders this was 35\% yield, which clearly shows 
the added value of this RNA-Seq approach [11]. It is beyond any doubt, that WGS and RNA-Seq will bring the paradigm shift started by WES to the next level: for a genetic diagnosis, geneticists and expert clinicians will no longer need to narrow down the differential clinical diagnosis to request sequencing of a specific gene or sets of genes, but all genetic information will be available at once from start. The results of this genetic analysis will directly guide the diagnostic patient evaluation, moving towards personalized patient care and treatment.

\section{Gene-based treatment options}

The identification of the gene defect in the patients has unfortunately not led to the anticipated major breakthrough in novel treatments. Only in few cases, a gene specific-treatment is possible and early start with these treatments prevents symptoms from occurring and can be lifesaving, as for example administration of thiamine to patients with fatal Leigh Syndrome due to SLC19A3 mutations [5]. In case of SLC25A32 mutations riboflavin is also the preferred treatment [12]. Our study revealed that SLC25A32 patients improved when treated with riboflavin as a vitamin B2 precursor of FAD which will compensate the lack of FAD functioning as a crucial co-factor in the mitochondrial transport chain [13]. In these cases, the genetic defect directly revealed a successful treatment option. In other cases, clinical improvement by specific treatments at a moment when the genetic defect was not known, revealed when the genetic defect was found, which patients might benefit from a comparable treatment. We demonstrated that again riboflavin could be beneficical for patients in case of ACAD9 defects [14]. Since increasing the levels of the riboflavin restored Complex I activity by compensating a disturbed ACAD9 and FAD binding or by stimulating Complex I assembly factors. However, not all ACAD9 patients are treatable with riboflavin [15]. A recent study on $A C A D 9$ disease-causing variants in 70 patients showed improvement of at least $20 / 31$ (65\%) ACAD9 deficient patients to riboflavin and $11 / 31$ (35\%) patients had no effect, although 20 patients were not treated and additional data about the treatment were missing for approximately 19 patients in this cohort. However, this justifies administration of riboflavin to every ACAD9 patient. Given the high frequency of ACAD9 deficiency, we also propose that early riboflavin administration for phenotypically consistent patients whilst their genetic investigations are still underway [16]. Another, successful treatment of infantileonset ACAD9-related cardiomyopathy was achieved by a combination of sodium 
Chapter 8

pyruvate, beta-blocker, and coenzyme $Q_{10}$ [17]. Finally, also a high fat diet resulted in clinical improvement in a patient with a OXPHOS Complex I deficiency due to a mutation in the TMEM126B gene [18].

Conclusively, still this is a minority, and gene and pathway based treatment strategies might fall short. More general, gene-correction/editing (CRISPR/Cas9) or cell-based strategies are more promising as a general treatment strategy, complemented by classical compound-screening strategies of the pharmaceutical industry. 


\section{References}

1. Gorman, G.S., et al., Prevalence of nuclear and mitochondrial DNA mutations related to adult mitochondrial disease. Ann Neurol, 2015. 77(5): p. 753-9.

2. Gorman, G.S., et al., Mitochondrial diseases. Nat Rev Dis Primers, 2016. 2: p. 16080.

3. Neveling, K., et al., A post-hoc comparison of the utility of sanger sequencing and exome sequencing for the diagnosis of heterogeneous diseases. Hum Mutat, 2013. 34(12): p. 1721-6.

4. Theunissen, T.E.J., et al., Whole Exome Sequencing Is the Preferred Strategy to Identify the Genetic Defect in Patients With a Probable or Possible Mitochondrial Cause. Front Genet, 2018. 9: p. 400.

5. Gerards, M., et al., Exome sequencing reveals a novel Moroccan founder mutation in SLC19A3 as a new cause of early-childhood fatal Leigh syndrome. Brain, 2013. 136(Pt 3): p. 882-90.

6. Kamps, R., et al., Genetic defects in mtDNA-encoded protein translation cause paediatric, mitochondrial cardiomyopathy with early-onset brain disease. Eur J Hum Genet, 2018. 26(4): p. 537-551.

7. Sallevelt, S., et al., Preimplantation genetic diagnosis for mitochondrial DNA mutations: analysis of one blastomere suffices. J Med Genet, 2017. 54(10): p. 693-697.

8. Theunissen, T.E.J., et al., Rapid Resolution of Blended or Composite Multigenic Disease in Infants by Whole-Exome Sequencing. J Pediatr, 2017. 182: p. 371-374 e2.

9. Clark, M.M., et al., Diagnosis of genetic diseases in seriously ill children by rapid whole-genome sequencing and automated phenotyping and interpretation. Sci Transl Med, 2019. 11(489).

10. Cummings, B.B., et al., Improving genetic diagnosis in Mendelian disease with transcriptome sequencing. Sci Transl Med, 2017. 9(386).

11. Kremer, L.S., S.B. Wortmann, and H. Prokisch, "Transcriptomics": molecular diagnosis of inborn errors of metabolism via RNA-sequencing. J Inherit Metab Dis, 2018. 41(3): p. 525-532.

12. Schiff, M., et al., SLC25A32 Mutations and Riboflavin-Responsive Exercise Intolerance. New England Journal of Medicine, 2016. 374(8): p. 795-797.

13. Hellebrekers, D., et al., Novel SLC25A32 mutation in a patient with a severe neuromuscular phenotype. Eur J Hum Genet, 2017. 25(7): p. 886888.

14. Gerards, M., et al., Riboflavin-responsive oxidative phosphorylation complex I deficiency caused by defective ACAD9: new function for an old gene. Brain, 2011. 134(Pt 1): p. 210-9.

15. Nouws, J., et al., ACAD9, a complex I assembly factor with a moonlighting function in fatty acid oxidation deficiencies. Hum Mol Genet, 2014. 23(5): p. 1311-9. 
Chapter 8

16. Repp, B.M., et al., Clinical, biochemical and genetic spectrum of 70 patients with ACAD9 deficiency: is riboflavin supplementation effective? Orphanet J Rare Dis, 2018. 13(1): p. 120.

17. Kadoya, T., et al., Successful treatment of infantile-onset ACAD9-related cardiomyopathy with a combination of sodium pyruvate, beta-blocker, and coenzyme Q10. J Pediatr Endocrinol Metab, 2019. 32(10): p. 11811185.

18. Theunissen, T.E.J., et al., Selection and Characterization of Palmitic Acid Responsive Patients with an OXPHOS Complex I Defect. Frontiers in Molecular Neuroscience, 2017. 10. 



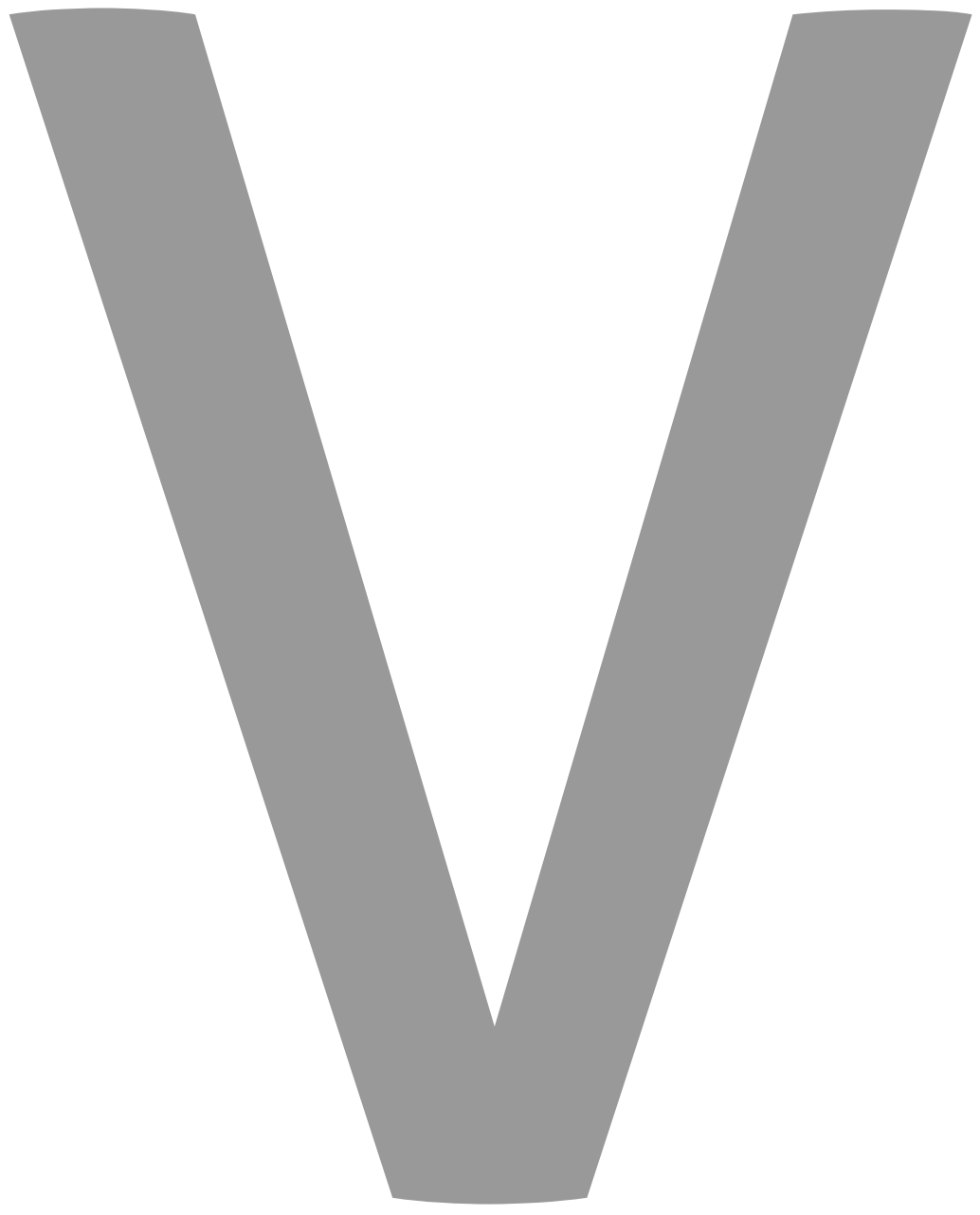


Part V

Appendix 

Nederlandse samenvatting 
Mitochondriële ziekten zijn het gevolg van een verminderde energiecapaciteit in de mitochondriën als gevolg van deficiënties in oxidatieve fosforylering (OXPHOS). Ze worden gekenmerkt door klinisch zeer heterogene en weefselspecifieke symptomen, die meestal weefsels en organen met hoge energiebehoeften, zoals hersenen, hart en spieren, raken. Aangezien het OXPHOS-systeem onder dubbele genetische controle staat, kunnen mutaties in zowel het mitochondriële DNA als het nucleaire DNA leiden tot een mitochondriële ziekte (hoofdstuk 1). Voornamelijk door de grote genetische heterogeniteit (mogelijk meer dan 1500 betrokken genen) en klinische variabiliteit, was bij de meerderheid van de patiënten een genetische oorzaak onbekend bij het begin van mijn onderzoek. Het eerste doel van het in dit proefschrift beschreven werk was dan ook het identificeren en functioneel karakteriseren van de onderliggende genetische oorzaak in het mtDNA of in nucleaire genen bij patiënten met OXPHOSaandoeningen. Op basis van een genetische diagnose kan counseling worden aangeboden aan de familie en prenatale of pre-implantatie-diagnostiek om de overdracht van de mitochondriële aandoening naar nakomelingen te voorkomen. In het geval van een mtDNA ziekte is dit minder eenvoudig dan bij nucleaire gendefecten, aangezien er tijdens de oögenese een mitochondriële bottleneck optreedt, die het mutatiepercentage en het ziekterisico bij de nakomelingen vaak onvoorspelbaar maakt. Hoewel cruciaal voor het begrijpen van de rol van mitochondriën bij oǒgenese, embryogenese en de overdracht van mtDNA ziektes, is het mechanisme van de mitochondriële bottleneck grotendeels onbekend, ook omdat geschikte diermodellen ontbreken. Het tweede doel van ons werk was dan ook het ontwikkelen van een diermodel voor het bestuderen en manipuleren van de mitochondriële bottleneck.

In hoofdstuk 2 wordt next generatie sequencing (NGS) technologie beschreven en de impact, die NGS heeft op het identificeren van pathogene varianten die genetische ziekten veroorzaken of van genetische risicofactoren. De mogelijkheden en beperkingen van verschillende technologieën en diagnostische benaderingen (d.w.z. gerichte NGS, sequentiebepaling van het gehele exoom (Whole Exome Sequencing - WES), RNA-Seq en sequentiebepaling van het gehele genoom (WGS)) worden besproken. Het gebruik van NGS-technologie in de genetische diagnostiek is de afgelopen decennia enorm toegenomen, mede 
dankzij aanzienlijke verbeteringen in betrouwbaarheid, sequencing-chemie, analyse-pijplijnen, data-interpretatie en kosten. Dit maakt NGS de methode, die bij voorkeur wordt gebruikt niet alleen binnen de klinische genetica, maar ook daarbuiten.

Een aantal klinische toepassingen van NGS wordt besproken, zoals de detectie van de oorzakelijke mutaties in erfelijke kankersyndromen door DNA sequencing en van splice-varianten door RNA sequencing. Toepassingen in het kader van preimplantatie genetische diagnostiek, somatische mutatieanalyse bij kanker, farmacogenetica, op basis van DNA uit bloed of liquid biopsies worden beschreven. Klinische beperkingen en ethische overwegingen met betrekking tot het gebruik van NGS voor deze toepassingen worden bediscussieerd. Hoewel dit hoofdstuk een meer algemene klinische toepassing van NGS behandelt, is het duidelijk dat NGS zeer relevant is voor de neurologische en mitochondriële ziekten, die het belangrijkste onderwerp van dit proefschrift zijn.

NGS is met succes gebruikt om het primaire onderliggende genetische defect bij de klinisch en genetisch zeer heterogene erfelijke mitochondriële ziekten te bepalen, wat een snelle introductie in de diagnostiek en klinische zorg voor deze ziekten heeft vergemakkelijkt (hoofdstuk 2). WES als eerste onderzoek, identificeert alle varianten in het coderende deel van het genoom, waardoor ook risicofactoren kunnen worden geïdentificeerd, die het risico op een ziekte alleen of in combinatie met een primair genetisch defect of andere risicofactoren kunnen verhogen. Het is waarschijnlijk dat genetische risicofactoren betrokken zijn bij mitochondriële ziektes, wat blijkt uit de zeer variabele manifestatie van bijv. de m.3243A>G-mutatie of het grotere percentage mannelijke patiënten met Leber erfelijke optische neuropathie (LHON). Nog steeds wordt bij een substantieel deel van de patiënten met mitochondriële ziekten geen primaire genetische oorzaak geïdentificeerd, wat betekent dat het ofwel wordt gemist door WES of dat de ziekte niet wordt veroorzaakt door een enkel primair genetisch defect. Een combinatie van meerdere genetische risicofactoren, gedefinieerd door een polygene risicoscore, zou een alternatieve verklaring kunnen zijn, zoals bijvoorbeeld is aangetoond op het gebied van kanker. Deze polygene scores worden berekend op basis van de genetische risico's van bekende met de ziekte gebaseerde SNP's uit GWAS-gegevens. In hoofdstuk 3 beschrijven we als proof-of-concept de 
identificatie en bijdrage van genetische risicofactoren in kandidaatgenen aan de ernst van klinische manifestatie bij het Lynch syndroom. Een vergelijkbare benadering zou kunnen worden toegepast om genetische factoren te identificeren die de heterogene klinische manifestatie van defecten in het mtDNA of nucleaire mitochondriële genen kunnen verklaren.

In hoofdstuk 3 gebruikten we gerichte NGS om zeldzame varianten te detecteren als mogelijke genetische modifiers in het Lynch syndroom door een panel van kandidaat-genen te analyseren in 35 Lynch syndroom mutatiedragers met een slecht klinisch beeld. Het genenpaneel bestond uit 154 genen die betrokken zijn bij de endometrium-fysiologie en carcinogenese. Data-analyse werd uitgevoerd met behulp van een pipeline, waarin varianten werden geselecteerd met een allelfrequentie lager dan 0,001 die tot niet-conservatieve aminozuursubstituties leiden. In totaal werden 54 varianten (categorieën $3=$ VUS of $4=$ waarschijnlijk pathogeen of 5 = pathogeen) beschouwd als risico-modifiers. Risicovoorspelling op basis van deze varianten is moeilijk te valideren, maar we voorspelden potentiële risicovarianten en functionele eiwitveranderingen voor 6 bekende genen (RET; p.(Asn361Lys), CYP1A1; p.(Cys457*), IGF1R; p.(Thr758Met), NCOR2; p.(Ser1964Cys), COMT; p.(Lys159del), mTOR; p.(Met1313Leu). Logistische regressive-analyse op basis van twee of meer risico-modificerende varianten bij vrouwen met een primaire pathogene mutatie was geassocieerd met een slecht klinisch fenotype. Een vergelijkbare benadering zou kunnen worden toegepast om genetische modifiers in mitochondriële ziekten te karakteriseren. Verder zullen in silico-modellering en in-vitro en in-vivo studies nodig zijn om de impact van deze varianten op de eiwitfunctie en cellulaire OXPHOS-processen te onderzoeken. De rol van genetische modifiers bij patiënten met mitochondriële ziekten zal relevant zijn binnen de groep zonder een genetische diagnose of als een risicofactor in de ernst van de ziektemanifestatie en zou tot mogelijk nieuwe behandelingsopties in de toekomst kunnen leiden.

Een zoektocht naar genetische modifiers zal de zoektocht naar primaire genetische oorzaken in families met mitochondriële ziekten aanvullen, zoals beschreven in hoofdstuk 4 en $\mathbf{5}$. De diagnose in deze families was gebaseerd op klinische symptomen, waaronder MRI en deficiënties in de OXPHOS-complexen in spieren of fibroblasten van patiënten. Bewijs voor de pathogeniciteit van nieuwe 
varianten en nieuwe genen werd geleverd door complementatie-testen. Leigh syndroom (hoofdstuk 4) is een vroege, maar vaak een fatale, progressieve neurodegeneratieve aandoening, die wordt veroorzaakt door mutaties in het mitochondriële of nucleaire DNA. Tot nu toe zijn er mutaties in meer dan 60 genen gerapporteerd, die Leigh syndroom veroorzaken, wat wijst op een extreme genetische heterogeniteit voor deze aandoening, maar die nog steeds slechts een deel van de gevallen verklaren. WES is gecombineerd met homozygositeitsbepaling als een extra filterstap om het onderliggende genetische defect te identificeren bij een Marokkaanse familie met fataal Leigh syndroom op vroege kinderleeftijd en specifieke MRI-afwijkingen in de hersenen. We ontdekten een homozygote nonsense mutatie (c.20C>A; p.Ser7Ter) in de thiamine-transporter SLC19A3. In vivo over-expressie van wild-type SLC19A3 toonde een verhoogde thiamine-opname, terwijl over-expressie van mutant SLC19A3 dat niet deed, wat bevestigt dat de mutaties resulteerden in een afwezig of niet-functioneel eiwit. Zeventien andere patiënten met Leigh syndroom werden gescreend op mutaties in SLC19A3 gen met behulp van conventionele Sanger sequencing. Twee nietverwante patiënten, beide van Marokkaanse afkomst en één ook van bloedverwante ouders, waren homozygoot voor dezelfde p.Ser7Ter mutatie. Een van deze patiënten vertoonde dezelfde MRI-afwijkingen als de patiënten uit de eerste familie. Opvallend was dat de patiënten die thiamine kregen een verbeterde levensverwachting hadden. Eén patiënt uit de derde familie verslechterde na onderbreking van de thiamine behandeling en herstelde na herstart. Hoewel niet verwant, kwamen alle patiënten uit de provincie Al Hoceima in Noord-Marokko. Op basis van genetisch markeronderzoek werd geschat dat de mutatie 1250-1750 jaar geleden is ontstaan. Onze resultaten toonden aan dat het SLC19A3 gen een nieuw kandidaatgen is voor mutatie-screening bij patiënten met Leigh syndroom, die baat zouden kunnen hebben bij hoge doses thiamine. Vooral Marokkaanse Leigh patiënten moeten worden getest op de c.20C $>A$ foundermutatie in SLC19A3.

In hoofdstuk 5 beschrijven we de toepassing van WES bij drie kinderen met cardiomyopathie en een hersenziekte met OXPHOS-deficiëntie op jonge leeftijd. De hersenpathologie werd bestudeerd door MRI-analyse. Bij consanguïne patiënt 1 identificeerden we een homozygote intron-variant (c.850-3A>G) in het $Q R S L 1$ 
Appendix

gen, waarvan werd voorspeld dat het abnormale splicing zou veroorzaken. De variant segregeerde met de ziekte in de familie en had een negatief effect op de eiwitfunctie, wat werd bevestigd door complementatie-onderzoeken. De OXPHOS functie werd alleen hersteld met wild-type QRSL1 en niet met de mutant. Patiënt 2 was compound heterozygoot voor twee nieuwe ziekteverwekkende varianten (c. [253G>A]; [938G>A]) in het MTO1 gen. Bij patiënt 3 ontdekten we een onbekende ziekteverwekkende variant (c.2872C>T) en een bekende ziekteverwekkende variant (c.1774C > T) in het $A A R S 2$ gen. De c.1774C $>$ T variant was aanwezig in de paternale kopie van het $A A R S 2$ gen, de c.2872C $>\mathrm{T}$ in de maternale kopie. Alle genen spelen een rol bij de vertaling van mtDNA gecodeerde eiwitten. Defecten in mtDNA gecodeerde eiwitvertaling leiden tot ernstige pediatrische cardiomyopathie en hersenziekte met OXPHOS-afwijkingen. Dit suggereert dat het hart en de hersenen bijzonder gevoelig zijn voor defecten in de mitochondriële eiwitsynthese tijdens de late embryonale of vroege postnatale ontwikkeling, waarschijnlijk als gevolg van de sterke mitochondriële biogenese die in dat stadium optreedt. Als zowel het hart als de hersenen betrokken zijn, is de prognose slecht met een waarschijnlijk dodelijke afloop op jonge leeftijd.

In de families, beschreven in hoofdstuk 4 en 5, maar ook in vele andere, zoals vermeld in hoofdstuk 2, hebben we het belang aangetoond van NGS en WES bij genetische onderzoek bij mitochondriële ziekten, waardoor de diagnostische opbrengst van $25 \%$ naar $69 \%$ is gestegen en de doorlooptijd van jaren tot maanden is gereduceerd. Bovendien identificeerden we in $30 \%$ van de gevallen defecten in nieuwe genen, wat aangeeft dat WES in combinatie met RNA-Seq nog steeds superieur is aan op genpanels gebaseerde benaderingen bij mitochondriële ziekten.

Ons tweede doel was het ontwikkelen van een diermodel voor het bestuderen en manipuleren van de mitochondriële bottleneck. We hebben al eerder laten zien dat zebravis een uitstekend model is, omdat zowel de timing als de grootte van de bottleneck van vis tot mens zeer goed geconserveerd is. Zebravissen hebben grote aantallen nakomelingen en zijn gemakkelijk genetisch te manipuleren. Zowel de embryonale ontwikkeling als de oŏgenese kunnen tot in detail worden bestudeerd. Om de pathologie en mechanismen bij lage mtDNA kopie-aantallen te begrijpen, hebben we in hoofdstuk 6 de mtDNA bottleneck gemanipuleerd 
door de expressie van Tfam, een belangrijke regulator van mtDNA replicatie, tijdens de vroege ontwikkeling van zebravissen te verlagen. Reductie van Tfam expressie met behulp van een splice-morpholino resulteerde in een afname van $42 \% \pm 17 \%$ van het mtDNA kopie-aantal in zebravisembryo's op 4 dagen na bevruchting ( $4 \mathrm{dpf}$ ). Aangedane embryo's vertoonden een abnormale ontwikkeling van het oog, de hersenen, het hart en de spieren, evenals een afname van de ATP-productie met $50 \% \pm 22 \%$. Transcriptoom-analyse toonde een afname van eiwit-coderende transcripten van de heavy strand van het mtDNA, en een lagere expressie van genen die betrokken zijn bij de haem-productie en bij het metabolisme van metabolieten, gericht op het verhogen van rRNA- en tRNAsynthese in de nucleoli. Deze stress of compenserende respons lijkt echter tekort te schieten als pathologie zich manifesteert. De expressie van genen die van belang zijn bij de oogontwikkeling is ook sterk verlaagd. Deze studie toont het belang aan van voldoende mtDNA-kopieën voor de vroege ontwikkeling van zebravissen. Zebravis blijkt een uitstekend model om de mtDNA bottleneck te manipuleren en het effect ervan op de embryogenese snel en bij grote aantallen nakomelingen te bestuderen.

In hoofdstuk 7 bediscussiëren wij de impact van NGS, niet alleen in de diagnose van mitochondriële ziekten, maar ook voor personalised medicine in het algemeen. Het staat buiten kijf dat WGS in combinatie met RNA-Seq de door WES gestarte paradigmaverschuiving naar het volgende diagnostische niveau zal brengen. De noodzaak om via uitgebreid klinisch onderzoek het genetisch onderzoek richting te geven naar een specifiek gen of sets van genen zal sterk verminderen, immers alle genetische informatie zal vanaf het begin in één keer beschikbaar zijn. De resultaten van deze genetische analyse zullen direct de diagnostische patiënt-evaluatie sturen en gepersonaliseerde patiëntenzorg en behandeling bij mitochondriële ziekten mogelijk maken. Volledige analyse van zowel het mtDNA als het exoom genereerde een diagnostische opbrengst van $69 \%$, verkregen in maanden, wat de $25 \%$ opbrengst, die met behulp van conventionele, gerichte sequencing wordt bereikt, verre overschrijdt. Zoals eerder aangegeven, is er nog steeds verbetering mogelijk voor het ontrafelen van de genetische oorzaak van de niet-gediagnosticeerde gevallen middels WGS, RNASeq of polygene risicoscores bij deze onopgeloste mitochondriële patiënten. 
Appendix

Aangezien we erin zijn geslaagd het eerste tuneable zebravismodel te ontwikkelen om de mtDNA bottleneck in de embryogenese te bestuderen, hebben we de impact die dit model zal hebben op het karakteriseren en manipuleren van het mitochondriële bottleneck en de processen die worden veroorzaakt door een verminderd mtDNA kopie-aantal in embryo's van zebravissen, verder bediscussieerd. Als we mtDNA mutaties in die zebravissen kunnen genereren, dan wordt dit een uitstekend model voor het testen van de huidige drie verschillende mitochondriële bottleneck-theorieën, voor het identificeren van mogelijke selectie-mechanismen en voor een betere risicobeoordeling van transmissie voor mtDNA ziekte. 


\section{Acknowledgements}

This thesis is by no means the work of a single individual. I'm grateful for the help of my direct colleagues, our collaborators, our students, but especially all the patients who have contributed to the contents of this thesis for possible treatment strategies in the future. Especially, my promotor, Prof. dr. H.J.M. Smeets, Bert who is responsible for the contents of this thesis, but rather for its very true existence, thanks for your patience and guidance in this educational odyssey. Dr. F.H.J. van Tienen, my co-promotor, Florence our assurance in checking the obtained novel results and reviewing the articles. My former group members are still colleagues for life; Andrea, Bert, Guido, Patrick, Marjolein, Ton, Ayşe, Chamindie, Christa, Irmgard, Ulrike, Andreas, Harma, Jaap, Emma, Lieve, Rita, An, Konny, Balazs, Bart and Iris for all academic support during the past years. Our excellent secretaries; currently Diana, and in the past Rosy, Els en Henny, thanks all of you! The Diagnostics and Research Department for Mitochondrial Genetics; Debby, Suzanne, Bianca, Eveline, René, Fons, Mike, Patrick, Ralph, thanks all of you! Also, all my friends who have contributed to this thesis indirectly for many years. Finally, I'm most really grateful to my wife Sandra, and my son Michael, my home family. Both made this PhD thesis directly possible after a long period stimulating me and supporting till the finish, thank you very much for this milestone, I love you both and and stay healthy! 



\section{Dankwoord}

Dit proefschrift is zeker niet het werk van een enkel individu. Ik ben dankbaar voor de hulp van mijn directe collega's, onze medewerkers, onze studenten, maar tevens alle patiënten die hebben bijgedragen aan de inhoud van dit proefschrift voor mogelijke behandelstrategieën in de toekomst. Vooral mijn promotor, Prof. dr. H.J.M. Smeets, Bert die verantwoordelijk is voor de inhoud van dit proefschrift, maar eerder voor het echte bestaan ervan, bedankt voor je geduld en begeleiding in deze leerzame odyssee. Dr. F.H.J. van Tienen, mijn co-promotor, Florence onze zekerheid bij het controleren van de verkregen nieuwe resultaten en het herzien van de artikelen. Mijn voormalige groepsleden zijn nog steeds collega's voor het leven; Andrea, Bert, Guido, Patrick, Marjolein, Ton, Ayşe, Chamindie, Christa, Irmgard, Ulrike, Andreas, Harma, Jaap, Emma, Lieve, Rita, An, Konny, Balazs, Bart en Iris voor alle academische ondersteuning van de afgelopen jaren. Onze uitstekende secretaresses; momenteel Diana, en in het verleden Rosy, Els en Henny, allemaal bedankt! De diagnostiek en research afdeling voor mitochondriële genetica; Debby, Suzanne, Bianca, Eveline, René, Fons, Mike, Patrick, Ralph, bedankt allemaal! Ook al mijn vrienden die al vele jaren indirect aan dit proefschrift hebben bijgedragen. Ten slotte ben ik mijn vrouw Sandra en mijn zoon Michael, mijn thuisfamilie, heel erg dankbaar. Beiden maakten dit proefschrift direct mogelijk na een lange periode van stimulering en ondersteuning tot aan de finish, hartelijk dank voor deze mijlpaal, ik hou van jullie beiden en blijf gezond! 



\section{Curriculum Vitae}

\section{Personal details:}

$\begin{array}{ll}\text { Name } & : \text { Ricky Kamps } \\ \text { Address } & : \text { Mgr. Van Gilsstraat 45D } \\ & 6461 \text { VE Kerkrade } \\ \text { Date of Birth } & : \text { : } 22 \text { December } 1972 \\ \text { Born } & : \text { Heerlen } \\ \text { Nationality } & : \text { Dutch } \\ \text { Gender } & : \text { Male } \\ \text { Language } & : \text { Dutch, English, and German } \\ \text { Telephone } & : \text { 0031627229571 } \\ \text { Email } & : \text { rick.kamps@maastrichtuniversity.nl or rick.kamps@home.nl } \\ \text { Social Media } & : \text { https://www.linkedin.com/in/rick-kamps }\end{array}$

\section{Education:}

September 2012 - November 2020

$\mathrm{PhD}$ candidate in the Department of Genetics and Cell Biology/Toxicogenomics, Maastricht University, Maastricht, the Netherlands. Thesis title: "Resolving the role of genetic defects and mtDNA copy number in mitochondrial disease and development". Promotor: Prof. dr. H.J.M. Smeets, Health, Medicine and Life Sciences, Department of Genetics and Cell Biology/ Clinical Genomics/GROW en MHeNS. Co-promotor: Dr. F.H.J. van Tienen, Medicine and Life Sciences, Department of Genetics and Cell Biology/Clinical Genomics/MHeNS.

September 2006 - February 2009:

Bachelor for Molecular Biology in Applied Sciences, Hogeschool Utrecht, Utrecht, the Netherlands

September 1993 - July 1997:

Long Education Medical Laboratory Technology on Middle Professional Education, Sittard, the Netherlands 
Appendix

September 1989 - July 1993:

Secondary School (HAVO), Antonius Doctor College (ADC), Kerkrade, the Netherlands

September 1985 - July 1989:

Secondary School (MAVO), MAVO-Heyendaal, Kerkrade, the Netherlands

\section{Courses:}

1. 2019: Illumina Next-Generation Sequencing (NGS) Training (NovaSeq, MiniSeq, and ISeq), Maastricht University, the Netherlands

2. 2019: Laboratory Animal Science Course, Maastricht University, the Netherlands

3. 2019: International Zebrafish and Medaka Course, Karlsruhe University, Germany

4. 2018: Nature Master Class, Scientific Writing and Publishing, Duesseldorf University, Germany

5. 2016: Grants for Individuals Spring Course, Maastricht University, the Netherlands

6. 2016: PacBio Single Molecule Real-Time NGS (SMRT-NGS) Training, Leiden University, the Netherlands

7. 2015: Advanced Academic Writing Course, Maastricht University, the Netherlands

8. 2015: Illumina Next-Generation Sequencing (NGS) Training (NextSeq), Maastricht University, the Netherlands

9. 2014: Illumina NGS Training (MiSeq), Maastricht University, the Netherlands

10. 2013: Illumina NGS Training (MiSeq), Liege University, Belgium

11. 2012: Illumina NGS Training (HiSeq), Maastricht University, the Netherlands

12. 2012: the Netherlands Bioinformatics Centre (NBIC) Data Training Radboud, Nijmegen University, the Netherlands

13. 2011: Roche FLX 454 Bioinformatics-Course in NGS Analysis, Maastricht University, the Netherlands

14. 2010: 1th International Short-Course in NGS, Breda Hogeschool Avans, the Netherlands

15. 2008: Nano-Technology Workshop, Athens University, Greece

16. 2007: Nano-Biology Workshop, Karlsruhe University, Germany

17. 2005: Stem-Cells, Theory and Practical, Homburg/Saar University, Germany

18. 2004, 2002, 1999: Immunological Histological Chemistry (IHC), Muenster University, Germany

\section{Current employment and work experience since graduating:}

August 2010 - present: 
Maastricht Academic Medical Centre (MUMC+), Maastricht University, Department of Genetics and Cell Biology/Toxicogenomics, Faculty of Health Science, the Netherlands

Research Institute CARIM, as head research engineer and PhD candidate April 2006 - July 2010:

Philips Research, Eindhoven, the Netherlands

Department of Molecular Diagnostics, as research engineer

August 2002 - March 2006:

Academic Hospital Maastricht, the Netherlands

Department of Pathology, Faculty of Medicine, Research Institute GROW, as research engineer

November 1997 - July 2002:

University RWTH Aachen, Germany

Department of Molecular and Cellular Anatomy as research engineer July 1997 - October 1997:

Hospital Atrium Heerlen, the Netherlands

Department of Clinical Chemistry Laboratory as diagnostic engineer 



\section{List of Publications}

1. Auke BC Otten\#, Rick Kamps\#, Patrick Lindsey, Mike Gerards, Hélène Pendeville-Samain, Marc Muller, Florence HJ van Tienen*, Hubert JM Smeets*. Tfam Depletion Leads to a Strong Reduction in mtDNA Copy Number OXPHOS Deficiency and Developmental Abnormalities in Zebrafish Embryos. Frontiers in Cell and Developmental Biology. 2020, Jun 12;8:381. doi: 10.3389/fcell.2020.00381. eCollection 2020. Impact factor $=5.20$

2. Maral Azodi", Rick Kamps", Stephane Heymans, Emma Louise Robinson. Topical Collection "Regulatory RNAs in Cardiovascular Development and Disease"Title: The Missing 'Inc' between Genetics and Cardiac Disease. Noncoding RNA. 2020, Jan14;6(1). Pii:E3.doi: 10.3390/ncrna6010003

3. Theunissen TEJ, Nguyen M, Kamps R, Hendrickx AT, Sallevelt SCEH, Gottschalk RWH, Calis CM, Stassen APM, de Koning B, Mulder-Den Hartog ENM, Schoonderwoerd K, Fuchs SA, Hilhorst-Hofstee $Y$, de Visser $M$, Vanoevelen J, Szklarczyk R, Gerards M, de Coo IFM, Hellebrekers DMEI, Smeets HJM. Whole Exome Sequencing Is the Preferred Strategy to Identify the Genetic Defect in Patients With a Probable or Possible Mitochondrial Cause. Front Genet. 2018, Oct 12; 9:400. Doi: 10.3389/fgene.2018.00400. eCollection 2018, Impact factor $=4.15$

4. Kamps R, Szklarczyk R, Theunissen TE, Hellebrekers DMEI, Sallevelt SCEH, Boesten IB, de Koning B, van den Bosch BJ, Salomons GS, SimasMendes M, Schoonderwoerd K, de Coo IFM, Vanoevelen JM, Smeets HJM. Genetic defects in mtDNA-encoded protein translation cause paediatric, mitochondrial cardiomyopathy with early-onset brain disease. Eur J Hum Genet. 2018, Apr;26(4):537-551. doi: 10.1038/s41431-017-0058-2. Epub 2018 Feb 13. Impact factor $=4.28$

5. Geraets IME, Chanda D, van Tienen FHJ, van den Wijngaard A, Kamps R, Neumann D, Liu Y, Glatz JFC, Luiken JJFP, Nabben M. Human embryonic stem cell-derived cardiomyocytes as an in vitro model to study cardiac insulin resistance. Biochim Biophys Acta Mol Basis Dis. 2018, May 1864(5 Pt B):1960-1967. Doi: 10.1016/j.bbadis.2017.12.025. Epub 2017 Dec 20. Review.PMID:29277329, Impact factor $=5.11$

6. Theunissen TEJ, Gerards M, Hellebrekers DMEI, van Tienen FH, Kamps $\mathbf{R}$, Sallevelt SCEH, Hartog ENMM, Scholte HR, Verdijk RM, Schoonderwoerd K, de Coo IFM, Szklarczyk R, Smeets HJM. Selection and Characterization of Palmitic Acid Responsive Patients with an OXPHOS Complex I Defect. Front Mol Neurosci. 2017, Oct 18; 10:336. Doi: 10.3389/fnmol.2017.00336. eCollection 2017. PMID: 29093663 Impact factor $=3.92$

7. Kamps R, Brandão RD, Bosch BJ, Paulussen AD, Xanthoulea S, Blok MJ, Romano A. Next-Generation Sequencing in Oncology: Genetic Diagnosis, Risk Prediction and Cancer Classification. Int J Mol Sci. 2017, Jan 31; 18(2). Pii: E308. Doi: 10.3390/ijms18020308. Impact factor $=3.29$

8. Theunissen TE, Sallevelt SC, Hellebrekers DM, de Koning B, Hendrickx AT, van den Bosch BJ, Kamps R, Schoonderwoerd K, Szklarczyk R, Mulder-Den Hartog EN, de Coo IF, Smeets HJ. Rapid Resolution of Blended or Composite Multigenic Disease in Infants by Whole-Exome 
Sequencing. J Pediatr. 2017, Jan 9. Pii: S0022-3476(16)31431-7. Doi: 10.1016/j.jpeds.2016.12.032. [Epub ahead of print]. Impact factor $=3.97$

9. Theunissen TE, Szklarczyk R, Gerards M, Hellebrekers DM, Mulder-Den Hartog EN, Vanoevelen J, Kamps R, de Koning B, Rutledge SL, SchmittMechelke T, van Berkel CG, van der Knaap MS, de Coo IF, Smeets HJ. Specific MRI Abnormalities Reveal Severe Perrault Syndrome due to CLPP Defects. Front Neurol. 2016, Nov 16; 7:203. eCollection 2016. Impact factor $=3.18$

10. Otten $A B$, Stassen $A P$, Adriaens $M$, Gerards $M$, Dohmen RG, Timmer AJ, Vanherle SJ, Kamps R, Boesten IB, Vanoevelen JM, Muller M, Smeets HJ. Replication Errors Made during Oogenesis Lead to Detectable De Novo mtDNA Mutations in Zebrafish Oocytes with a Low mtDNA Copy Number. Genetics. 2016, Dec; 204(4):1423-1431. Epub 2016 Oct 21 . Impact factor $=3.79$

11. Jóri $B$, Kamps R, Xanthoulea $S$, Delvoux B, Blok MJ, Van de Vijver $K$, de Koning B, Trups Oei F, Tops C, Speel EJ, Kruitwagen R, Gomez-Garcia EB, Romano A. Germ-line variants identified by next generation sequencing in a panel of estrogen and cancer associated genes correlate with poor clinical outcome in Lynch syndrome patients. Oncotarget. 2015, Dec 1; 6(38):41108-22. Doi: 10.18632/oncotarget.5694. Impact factor $=6.36$

12. Vrijenhoek T, Kraaijeveld K, Elferink M, de Ligt J, Kranendonk E, Santen G, Nijman IJ, Butler D, Claes G, Costessi A, Dorlijn W, van Eyndhoven W, Halley DJ, van den Hout MC, van Hove S, Johansson LF, Jongbloed JD, Kamps R, Kockx CE, de Koning B, Kriek M, Lekanne Dit Deprez $R$, Lunstroo $H$, Mannens M, Mook OR, Nelen M, Ploem C, Rijnen M, Saris J],Sinke R, Sistermans E, van Slegtenhorst M, Sleutels $F$, van der Stoep $\mathrm{N}$, van Tienhoven $M$, Vermaat $M$, Vogel M, Waisfisz Q, Marjan Weiss J, van den Wijngaard $A$, van Workum $W$, Ijntema $H$, van der Zwaag $B$, van IJcken WF, den Dunnen J,Veltman JA, Hennekam R, Cuppen E. Nextgeneration sequencing-based genome diagnostics across clinical genetics centers: implementation choices and their effects. Eur J Hum Genet. 2015, Sep; 23(9):1142-50. Doi: 10.1038/ejhg.2014.279. Epub 2015 Sep. Impact factor $=4.35$

13. Nguyen M, Boesten I, Hellebrekers DM, Vanoevelen J, Kamps R, de Koning B, de Coo IF, Gerards M, Smeets HJ. Pathogenic CWF19L1 variants as a novel cause of autosomal recessive cerebellar ataxia and atrophy. Eur J Hum Genet. 2015, Jul 22. Doi: 10.1038/ejhg.2015.158. PMID: 26197978. Impact factor $=4.35$

14. Gerards M, Kamps R, van Oevelen J, Boesten I, Jongen E, de Koning B, Scholte HR, de Angst I, Schoonderwoerd K, Sefiani A, Ratbi I,Coppieters W, Karim L, de Coo R, van den Bosch B, Smeets H. Exome sequencing reveals a novel Moroccan founder mutation in SLC19A3 as a new cause of early-childhood fatal Leigh syndrome. Brain. 2013, Mar; 136(Pt 3):882-90. Doi: 10.1093/brain/awt013. Epub 2013 Feb 18. Impact factor $=10.23$

15. Dassen H, Punyadeera C, Kamps R, Klomp J, Dunselman G, Dijcks F, de Goeij A, Ederveen A, Groothuis P. Olfactomedin-4 regulation by estrogen in the human endometrium requires epidermal growth factor signaling. Am J Pathol. 2010, Nov; 177(5):2495-508. PMID: 21048224 [PubMed - indexed for MEDLINE]. Impact factor $=4.59$ 
16. Dittmer WU, Evers TH, Hardeman WM, Huijnen W, Kamps R, de Kievit $P$, Neijzen JH, Nieuwenhuis JH, Sijbers MJ, Dekkers DW, Hefti MH, Martens MF. Rapid, high sensitivity, point-of-care test for cardiac troponin based on optomagnetic biosensor. Clin Chim Acta. 2010, Jun 3; 411 (1112):868-73. Epub 2010 Mar 6. PMID: 20211616 [PubMed - indexed for MEDLINE]. Impact factor $=2.39$

17. Punyadeera C, Kamps R, Defrère S, Dijcks F, de Goeij A, Ederveen A, Dunselman G, Groothuis P. Effects of selective oestrogen receptor modulators on proliferation in tissue cultures of pre- and postmenopausal human endometrium. J Steroid Biochem Mol Biol. 2008, Nov;112(13):102-9. Epub 2008 Sep 11. PMID: 18832036[PubMed - indexed for MEDLINE]. Impact factor $=2.83$

18. Nap AW, Groothuis PG, Punyadeera C, Klein-Hitpass L, Kamps R, Delvoux B, Dunselman GA. Oral contraceptives prevent the development of endometriosis in the chicken chorioallantoic membrane model. Contraception. 2008, Sep; 78(3):257-65. Epub 2008 Jul 9. PMID: 18692618 [PubMed - indexed for MEDLINE]. Impact factor $=2.83$

19. Dassen H, Kamps R, Punyadeera C, Dijcks F, de Goeij A, Ederveen A, Dunselman G, Groothuis P. Haemoglobin expression in human endometrium. Human Reproduction. 2008, Mar; 23(3):635-41. Epub 2008 Jan 23. PMID: 18216035 [PubMed - indexed for MEDLINE] Impact factor $=4.57$

20. Van Langendonckt A, Punyadeera C, Kamps R, Dunselman G, KleinHitpass L, Schurgers LJ, Squifflet J, Donnez J, Groothuis P. Identification of novel antigens in blood vessels in rectovaginal endometriosis. Mol Hum Reprod. 2007, Dec; 13(12):875-86. Epub 2007 Nov 6. PMID: 17989082 [PubMed - indexed for MEDLINE]. Impact factor=2.87

21. Dassen $H$, Punyadeera $C$, Kamps R, Delvoux B, Van Langendonckt $A$, Donnez J, Husen B, Thole H, Dunselman G, Groothuis P. Estrogen metabolizing enzymes in endometrium and endometriosis. Hum Reprod. 2007, Dec; 22(12):3148-58. Epub 2007 Oct 6. PMID: 17921479[PubMed - indexed for MEDLINE]. Impact factor $=3.77$

22. Feroze-Zaidi F, Fusi L, Takano M, Higham J, Salker MS, Goto T, Edassery S, Klingel K, Boini KM, Palmada M, Kamps R, Groothuis PG, Lam EW, Smith SK, Lang F, Sharkey AM, Brosens JJ. Role and regulation of the serum- and glucocorticoid-regulated kinase 1 in fertile and infertile human endometrium. Endocrinology. 2007, Oct; 148(10):5020-9. Epub 2007 Jul 19. PMID: 17640988 [PubMed - indexed for MEDLINE]. Impact factor $=4.50$

23. Dassen H, Punyadeera C, Kamps R et al. Progesterone regulation of implantation-related genes: new insights into the role of oestrogen. Cellular Molecular Life Science. 2007, April. Impact factor $=5.81$

24. Punyadeera C, Thijssen VL, Tchaikovski S, Kamps R, Delvoux B, Dunselman GA, de Goeij AF, Griffioen AW, Groothuis PG. Expression and regulation of vascular endothelial growth factor ligands and receptors during menstruation and post-menstrual repair of human endometrium. Mol Hum Reprod. 2006, Jun; 12(6):367-75. Epub 2006 Apr 28. PMID: 16648151 [PubMed - indexed for MEDLINE]. Impact factor $=2.87$

25. Punyadeera C, Dassen H, Klomp J, Dunselman G, Kamps R, Dijcks F, Ederveen A, de Goeij A, Groothuis P. Oestrogen-modulated gene 
expression in the human endometrium. Cell Mol Life Sci. 2005, Jan; 62(2):239-50. PMID: 15666095[PubMed - indexed for MEDLINE]. Impact factor $=4.58$

26. Punyadeera C, Dunselman G, Marbaix E, Kamps R, Galant C, Nap A, Goeij A, Ederveen A, Groothuis P. Triphasic pattern in the ex vivo response of human proliferative phase endometrium to oestrogens. J Steroid Biochem Mol Biol. 2004, Oct; 92(3):175-85. PMID: 15555911[PubMed - indexed for MEDLINE]. Impact factor $=2.72$

\section{Oral presentations}

1. October 2019: Cardiology World Congress,Tokyo, Japan

2. February 2019: International Clinical Genetics Congress MEDLAB, Dubai, United Arab Emirates

3. April 2018: International Nobel Peace Congress, Erzurum, Turkey

4. March 2016: Istanbul University Genetics-IUGEN, Istanbul Turkey

5. November 2015: Illumina Technical User Experience Day, Utrecht, the Netherlands

6. June 2011: Benelux Next-generation sequencing conference, Utrecht, the Netherlands

\section{Poster presentations}

1. November 2018: The Netherlands Mitochondria Meeting Vrije Universiteit Amsterdam, Amsterdam, the Netherlands

2. June 2017: Mitochondrial Congress-EUROMIT, Cologne, Germany

\section{Teaching}

1. Supervision of Biomedical Bachelor student, Senior Internship (2019, Bachelor thesis)

2. Supervision of Biomedical Master Student, Senior Internship (20182019, Master thesis)

3. Supervision of Biomedical Master Student, Junior Internship (2019, junior Report)

4. Supervision of Biomedical Master Student, Senior Internship (2017, Master thesis)

5. Supervision of Biomedical Bachelor student, Senior Internship (2012, Bachelor thesis)

6. Supervision of Biomedical Bachelor student, Senior Internship (2011, Bachelor thesis)

\section{Other interests}

Music, Football, Koi Fish... 